Homology, Homotopy and Applications, vol.9(2), 2007, pp.233-320

\title{
HELLER TRIANGULATED CATEGORIES
}

\author{
MATTHIAS KÜNZER \\ (communicated by Charles Weibel)
}

\begin{abstract}
Let $\mathcal{E}$ be a Frobenius category. Let $\underline{\underline{\mathcal{E}}}$ denote its stable category. The shift functor on $\underline{\underline{\mathcal{E}}}$ induces, by pointwise application, an inner shift functor on the category of acyclic complexes with entries in $\underline{\underline{\mathcal{E}}}$. Shifting a complex by 3 positions yields an outer shift functor on this category. Passing to quotient modulo split acyclic complexes, HeLLer remarked that inner and outer shift become isomorphic, via an isomorphism satisfying yet a further compatibility. Moreover, HELLER remarked that a choice of such an isomorphism determines a Verdier triangulation on $\underline{\underline{\mathcal{E}}}$, except for the octahedral axiom. We generalise the notion of acyclic complexes such that the accordingly enlarged version of Heller's construction includes octahedra.
\end{abstract}

\section{Contents}

0 Introduction $\quad \mathbf{2 3 4}$

0.1 Heller's idea . . . . . . . . . . . . . . . . . . . . . . . . . . 234

0.2 Definition of Heller triangulated categories . . . . . . . . . . . 236

0.3 A result to begin with . . . . . . . . . . . . . . . . . 239

0.4 A quasicyclic category . . . . . . . . . . . . . . . . . . 239

0.5 Some remarks . . . . . . . . . . . . . . . . . . . . . 239

0.6 Notations and conventions . . . . . . . . . . . . . . . . . . . . 240

1 Definition of a Heller triangulated category 242

1.1 Periodic linearly ordered sets and their strips . . . . . . . . . . . . . 242

1.2 Heller triangulated categories . . . . . . . . . . . . . . . . . . . . 244

2 Some equivalences 253

2.1 Some notation . . . . . . . . . . . . . . . . . 253

2.2 Density of the restriction functor from $\bar{\Delta}_{n}^{\#}$ to $\dot{\Delta}_{n} \ldots \ldots \ldots . \ldots 255$

2.3 Fullness of the restriction functor from $\bar{\Delta}_{n}^{\#}$ to $\dot{\Delta}_{n} \ldots \ldots \ldots \ldots . .259$

2.4 The equivalence between $\mathcal{C}^{+}\left(\bar{\Delta}_{n}^{\#}\right)$ and $\mathcal{C}\left(\dot{\Delta}_{n}\right) \ldots \ldots$. . . . . . . 259

2.5 Auxiliary equivalences . . . . . . . . . . . . . . 261

Received July 21, 2006, revised February 28, 2007; published on September 6, 2007.

2000 Mathematics Subject Classification: 18E30.

Key words and phrases: triangulated category.

Copyright (c) 2007, International Press. Permission to copy for private use granted. 
3 Verification of Verdier's axioms $\quad 266$

3.1 Restriction from $\mathcal{C}^{+, \vartheta=1}\left(\bar{\Delta}_{n}^{\#}\right)$ to $\mathcal{C}\left(\dot{\Delta}_{n}\right)$ is dense and full $\ldots \ldots 266$

3.2 An omnibus lemma . . . . . . . . . . . . . . . . . . 270

3.3 Turning $n$-triangles . . . . . . . . . . . . . . . . . . . 272

3.4 Application to the axioms of Verdier . . . . . . . . . . . . . . 273

$3.5 n$-triangles and strictly exact functors _ . . . . . . . . . . 274

3.6 A remark on spectral sequences . . . . . . . . . . . . . . 275

4 The stable category of a Frobenius category is Heller triangulated 275

4.1 Definition of $\mathcal{F}^{\square}\left(\bar{\Delta}_{n}^{\#}\right)$, modelling $\underline{\underline{\mathcal{F}}}^{+}\left(\bar{\Delta}_{n}^{\#}\right) \ldots \ldots \ldots 275$

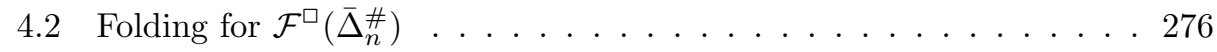

4.3 Some 1-epimorphic functors . . . . . . . . . . . . . . . . 278

4.4 Construction of $\vartheta \ldots \ldots \ldots$

4.5 Exact functors induce strictly exact functors . . . . . . . . . . . 285

5 Some quasicyclic categories $\quad 287$

5.1 The category $\mathcal{C}^{+}\left(\bar{\Delta}_{n}^{\#}\right)$ is Frobenius . . . . . . . . . . . . 287

5.2 A quasicyclic category . . . . . . . . . . . . . . . . . . . 294

5.3 A biquasicyclic category . . . . . . . . . . . . . . . . . . 294

A Some general lemmata $\quad 295$

A.1 An additive lemma . . . . . . . . . . . . . . . . . 295

A.2 Exact categories . . . . . . . . . . . . . . . . 296

A.3 Kernel-cokernel-criteria . . . . . . . . . . . . . . . . . . 300

A.4 An exact lemma . . . . . . . . . . . . . . . . . . . . . 302

A.5 Some abelian lemmata . . . . . . . . . . . . . . . . . 303

A.6 On Frobenius categories . . . . . . . . . . . . . . . . 307

A.7 Pointwise pullback and pushout . . . . . . . . . . . . . 315

A.8 1-epimorphic functors . . . . . . . . . . . . . 316

\section{Introduction}

\subsection{Heller's idea ${ }^{1}$}

0.1.1 Stable Frobenius CATEGORIES AND AN ISOMORPHISM BETWEEN OUTER AND INNER SHIFT

Let $\mathcal{E}$ be a Frobenius category, i.e. an exact category with enough bijective objects. For instance, the category of complexes with values in an additive category, equipped with pointwise split exact sequences, is a Frobenius category.

Let $\underline{\underline{\mathcal{E}}}$ denote the stable category of $\mathcal{E}$; see $\S 0.3$. Assume that $\underline{\underline{\mathcal{E}}}$ has split idempotents.

\footnotetext{
${ }^{1}$ Heller formulated his idea using Freyd categories. We will rephrase it using complexes, for this is the language we will use below. See $\S \S 0.2 .2,0.2 .4$.
} 
A complex with entries in $\underline{\underline{\mathcal{E}}}$ is acyclic if any Hom functor turns it into an acyclic complex of abelian groups. Let $\underline{\underline{\mathcal{E}}}^{+}\left(\bar{\Delta}_{2}^{\#}\right)$ denote ${ }^{2}$ the category of acyclic complexes with entries in $\underline{\underline{\mathcal{E}}}$. Let $\underline{\underline{\underline{\mathcal{E}}}}^{+}\left(\bar{\Delta}_{2}^{\#}\right)$ denote the homotopy category of the category $\underline{\underline{\mathcal{E}}}^{+}\left(\bar{\Delta}_{2}^{\#}\right)$ of acyclic complexes; that is, the quotient category of acyclic complexes modulo split acyclic complexes.

There is a shift automorphism $T$ on $\underline{\underline{\mathcal{E}}}$. It induces a first, inner shift automorphism $\mathrm{T}^{+}\left(\bar{\Delta}_{2}^{\#}\right)$ on $\underline{\underline{\mathcal{E}}}^{+}\left(\bar{\Delta}_{2}^{\#}\right)$ by pointwise application.

There is also a shift automorphism $T_{2}$ on the diagram $\bar{\Delta}_{2}^{\#}$. It induces a second, outer shift automorphism $\underline{\underline{\mathcal{E}}}^{+}\left(\mathrm{T}_{2}\right)$ on $\underline{\underline{\mathcal{E}}}^{+}\left(\bar{\Delta}_{2}^{\#}\right)$, shifting a complex by three positions.

Both outer and inner shift induce automorphisms

$$
\underline{\underline{\underline{\mathcal{E}}}}^{+}\left(\mathrm{T}_{2}\right) \text { resp. } \underline{\mathrm{T}^{+}\left(\bar{\Delta}_{2}^{\#}\right)} \text { on } \underline{\underline{\underline{\mathcal{E}}}}+\left(\bar{\Delta}_{2}^{\#}\right) .
$$

HELLER remarked that these functors are isomorphic. But there is no a priori given isomorphism. So he chose an isomorphism

$$
\underline{\underline{\underline{\mathcal{E}^{+}}}\left(\mathrm{T}_{2}\right)} \stackrel{\vartheta_{2}}{\sim} \mathrm{T}^{+}\left(\bar{\Delta}_{2}^{\#}\right)
$$

satisfying, for technical reasons, still a further compatibility.

Then he remarked that the choice of such an isomorphism $\vartheta_{2}$ determines a triangulation on $\underline{\underline{\mathcal{E}}}$ in the sense of PUPPE [26, Sec. 2]; that is, it satisfies all the axioms of VERDIER [29, Def. 1-1] except possibly for the octahedral axiom. Namely, as distinguished triangles we take acyclic complexes on which outer and inner shift coincide (i.e., which are "3-periodic up to shift") and on which $\vartheta_{2}$ is the identity.

Whether this observation now fathoms Puppe triangulations remains to be discussed. Whenever two objects are isomorphic but lack a nature-given isomorphism, it is at any rate not unusual to pick an isomorphism. Once a suitable isomorphism between our shift functors is chosen, a Puppe triangulation ensues. In nontechnical terms, we may let the relation between the two shifts govern the Puppe triangulations. This is a possible point of view, which we shall adopt and put into a larger framework (see $\S 0.2 .2$ ).

Heller used this construction to parametrise Puppe triangulations on $\underline{\underline{\mathcal{E}}}$. The non-uniqueness of such a Puppe triangulation on $\underline{\underline{\mathcal{E}}}$, and hence the impossibility of an intrinsic definition of distinguished triangles thus can be regarded as rooted in the possible nontriviality of the automorphism group of the inner shift functor $\mathrm{T}^{+}\left(\bar{\Delta}_{2}^{\#}\right)$, or, by choice, of the outer shift functor $\underline{\underline{\mathcal{E}}}^{+}\left(\mathrm{T}_{2}\right)$. This is to be seen in contrast to the intrinsic characterisation of short exact sequences in an abelian category.

\subsubsection{The stable Frobenius Case models a general definition of Puppe TRIANGULATIONS}

A weak kernel in an additive category is defined by the universal property of a kernel, except for the uniqueness of the induced morphism (dually a weak cokernel).

\footnotetext{
${ }^{2}$ The notation using the diagram $\bar{\Delta}_{2}^{\#}$ is chosen to fit into a larger framework; see $§ 0.2 .2$ for more details.
} 
A weakly abelian category is an additive category in which each morphism has a weak kernel and a weak cokernel, and in which each morphism is a weak kernel and a weak cokernel. For instance, the stable category $\underline{\underline{\mathcal{E}}}$ appearing in $\S 0.1 .1$ is a weakly abelian category.

Let $\mathcal{C}$ be a weakly abelian category with split idempotents carrying a shift automorphism T. Now Heller's construction yields an alternative, equivalent definition of a Puppe triangulation on $(\mathcal{C}, \mathrm{T})$ as being an isomorphism

$$
\underline{\mathcal{C}^{+}\left(\mathrm{T}_{2}\right)} \stackrel{\vartheta_{2}}{\sim} \underline{\mathrm{T}^{+}\left(\bar{\Delta}_{2}^{\#}\right)}
$$

satisfying still a further compatibility. In other words, a Puppe triangulated category can be defined to be such a triple $\left(\mathcal{C}, \mathrm{T}, \vartheta_{2}\right)$.

\subsubsection{From Puppe to Verdier AND Beyond}

In a Puppe triangulated category, the octahedral axiom of Verdier [29, Def. 1-1] does not seem to hold in general. ${ }^{3}$

In a Verdier triangulated category, in turn, it seems to be impossible to derive the existence of the two extra triangles in a particular octahedron described in [3, 1.1.13], or to distinguish crosses as in [16, App.].

Moreover, to define a K-theory simplicial set of a triangulated category, one is inclined to take objects as 1-simplices, distinguished triangles as 2-simplices, distinguished octahedra as 3-simplices, etc.

So we enlarge the framework, generalising from $\mathcal{C}^{+}\left(\bar{\Delta}_{2}^{\#}\right)$ to $\mathcal{C}^{+}\left(\bar{\Delta}_{n}^{\#}\right)$, as described next in $\S 0.2$.

\subsection{Definition of Heller triangulated categories}

\section{2 .1 A DIAGRAM SHAPE}

Given $n \geqslant 0$, we let $\Delta_{n}:=\{i \in \mathbf{Z}: 0 \leqslant i \leqslant n\}$, considered as a linearly ordered set. Let $\bar{\Delta}_{n}$ be the periodic prolongation of $\Delta_{n}$, consisting of $\mathbf{Z}$ copies of $\Delta_{n}$ put in a row. This is a periodic linearly ordered set; that is, a linearly ordered set equipped with a shift automorphism $i \longmapsto i^{+1}$. For instance, $\bar{\Delta}_{2}=\left\{\ldots, 2^{-1}, 0,1,2\right.$, $\left.0^{+1}, \ldots\right\}$, equipped with $i \longmapsto i^{+1}$. Let $\overline{\boldsymbol{\Delta}}$ be the category consisting of periodic linearly ordered sets of the form $\bar{\Delta}_{n}$ as objects, and of monotone shift-compatible maps as morphisms.

Let $\bar{\Delta}_{n}\left(\Delta_{1}\right)$ denote the category of morphisms in $\bar{\Delta}_{n}$, i.e. the category of $\bar{\Delta}_{n^{-}}$ valued diagrams of shape $\Delta_{1}$. Given $\alpha, \beta \in \bar{\Delta}_{n}$ such that $\alpha \leqslant \beta$, the object $(\alpha \longrightarrow \beta)$ in $\bar{\Delta}_{n}\left(\Delta_{1}\right)$ is abbreviated by $\beta / \alpha$.

Let $\bar{\Delta}_{n}^{\#}$ be the full subcategory of $\bar{\Delta}_{n}\left(\Delta_{1}\right)$ that consists of objects $\beta / \alpha$ within a

\footnotetext{
${ }^{3}$ The author lacks an example of a category that is Puppe but not Verdier triangulated, but strongly suspects that such an example exists, i.e. that the octahedral axiom is not a consequence of Puppe's axioms; cf. Question 1.7. In any case, the truth of such a deduction is unknown.
} 
single period, i.e. such that $\beta^{-1} \leqslant \alpha \leqslant \beta \leqslant \alpha^{+1}$. For instance,

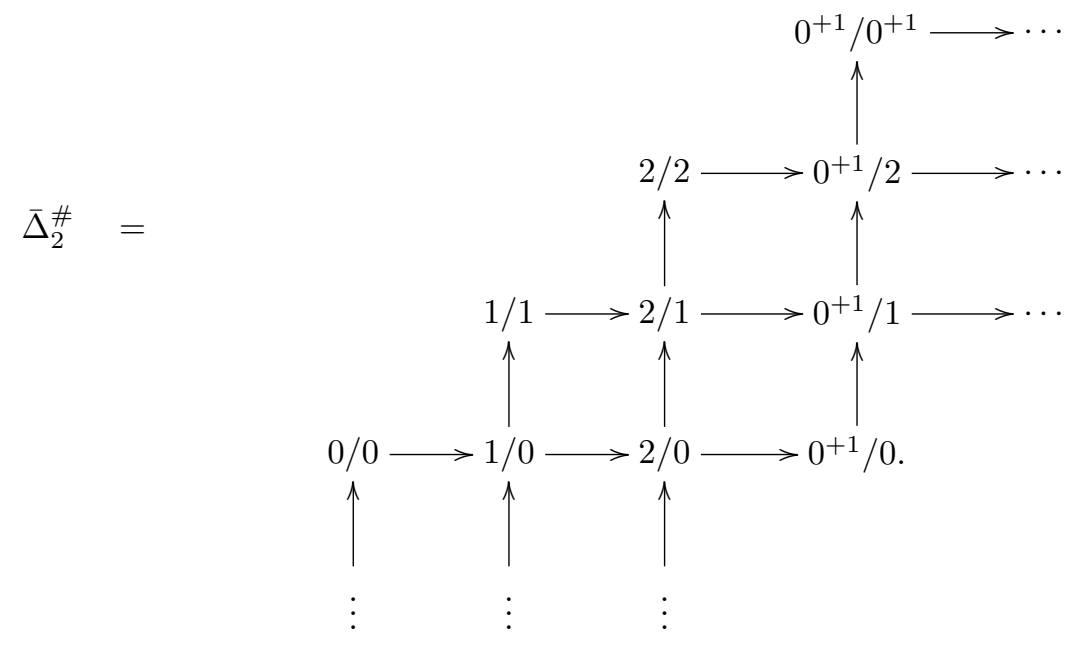

\subsubsection{HELler triangulations}

Let $\mathcal{C}$ be a weakly abelian category; see $\S 0.1 .2$. A sequence $X \stackrel{f}{\longrightarrow} Y \stackrel{g}{\longrightarrow} Z$ in $\mathcal{C}$ is called exact at $\boldsymbol{Y}$ if $f$ is a weak kernel of $g$, or, equivalently, if $g$ is a weak cokernel of $f$. A commutative quadrangle in $\mathcal{C}$ whose diagonal sequence is exact at the middle object is called a weak square.

Let $\mathcal{C}^{+}\left(\bar{\Delta}_{n}^{\#}\right)$ be the category of $\mathcal{C}$-valued diagrams of shape $\bar{\Delta}_{n}^{\#}$ with a zero at $\alpha / \alpha$ and at $\alpha^{+1} / \alpha$ for each $\alpha \in \bar{\Delta}_{n}$, and such that the quadrangle on $(\gamma / \alpha, \delta / \alpha, \gamma / \beta, \delta / \beta)$ is a weak square whenever $\delta^{-1} \leqslant \alpha \leqslant \beta \leqslant \gamma \leqslant \delta \leqslant \alpha^{+1}$. Let $\underline{\mathcal{C}^{+}\left(\bar{\Delta}_{n}^{\#}\right)}$ be the quotient of $\mathcal{C}^{+}\left(\bar{\Delta}_{n}^{\#}\right)$ modulo the full subcategory of diagrams therein that consist entirely of split morphisms.

For instance, $\mathcal{C}^{+}\left(\bar{\Delta}_{2}^{\#}\right)$ is the category of $\mathcal{C}$-valued acyclic complexes and $\mathcal{C}^{+}\left(\bar{\Delta}_{2}^{\#}\right)$ is its quotient modulo split acyclic complexes, i.e. the homotopy category of $\overline{\mathcal{C} \text {-valued }}$ acyclic complexes.

Furthermore, suppose given an automorphism $\mathrm{T}$ on $\mathcal{C}$. We obtain two shift functors on $\mathcal{C}^{+}\left(\bar{\Delta}_{n}^{\#}\right)$, the inner shift given by pointwise application of $\mathrm{T}$, and the outer shift induced by a diagram shift $j / i \longmapsto i^{+1} / j$.

A Heller triangulation on $(\mathcal{C}, T)$ is a tuple of isomorphisms $\vartheta=\left(\vartheta_{n}\right)_{n \geqslant 0}$, where $\vartheta_{n}$ is an isomorphism from the outer to the inner shift on $\mathcal{C}^{+}\left(\bar{\Delta}_{n}^{\#}\right)$. This tuple is required to be compatible with the functors induced by periodic monotone maps between $\bar{\Delta}_{n}$ and $\bar{\Delta}_{m}$, where $m, n \geqslant 0$. Moreover, it is required to be compatible with an operation called folding, which emerges from the fact that a weak square

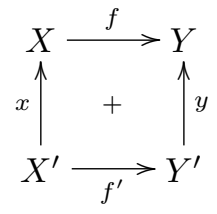


entails a folded weak square

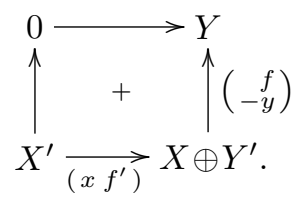

A Heller triangulated category is a triple $(\mathcal{C}, T, \vartheta)$ as just described, often just denoted by $\mathcal{C}$.

An $\boldsymbol{n}$-triangle in a Heller triangulated category $\mathcal{C}$ is an object $X$ of $\mathcal{C}^{+}\left(\bar{\Delta}_{n}^{\#}\right)$ that is periodic in the sense that outer shift and inner shift coincide on $X$, and that satisfies $X \vartheta_{n}=1$. The usual properties of 2 -triangles generalise to $n$-triangles.

If $\mathcal{C}$ is a Heller triangulated category in which idempotents split, then, taking the 2-triangles as the distinguished triangles, it is also triangulated in the sense of VERDiER [29, Def. 1-1]; see Proposition 3.6.

\subsubsection{StRICTLY EXACT FUNCTORS}

An additive functor $\mathcal{C} \stackrel{F}{\longrightarrow} \mathcal{C}^{\prime}$ between Heller triangulated categories $(\mathcal{C}, T, \vartheta)$ and $\left(\mathcal{C}^{\prime}, T^{\prime}, \vartheta^{\prime}\right)$ is called strictly exact if, firstly, it respects weak kernels, or, equivalently, weak cokernels; if, secondly, $F \mathrm{~T}^{\prime}=\mathrm{T} F$; and if, thirdly, the functor

$$
\underline{\mathcal{C}^{+}\left(\bar{\Delta}_{n}^{\#}\right)} \stackrel{F^{+}\left(\bar{\Delta}_{n}^{\#}\right)}{\longrightarrow} \underline{\mathcal{C}^{\prime+}\left(\bar{\Delta}_{n}^{\#}\right)},
$$

induced by pointwise application of $F$, satisfies $\underline{F^{+}\left(\bar{\Delta}_{n}^{\#}\right)} \star \vartheta_{n}^{\prime}=\vartheta_{n} \star F^{+}\left(\bar{\Delta}_{n}^{\#}\right)$ for $n \geqslant 0$.

\subsubsection{ENLARGE TO SIMPLIFY}

Let $\dot{\Delta}_{n}:=\{i \in \mathbf{Z}: 1 \leqslant i \leqslant n\}$. We have an embedding $\dot{\Delta}_{n} \longleftrightarrow \bar{\Delta}_{n}^{\#}$ via $\alpha \longmapsto \alpha / 0$. Let $\mathcal{C}$ be a weakly abelian category. Let $\mathcal{C}\left(\dot{\Delta}_{n}\right)$ denote the category of $\mathcal{C}$-valued diagrams of shape $\dot{\Delta}_{n}$. Let $\mathcal{C}\left(\dot{\Delta}_{n}\right)$ be the quotient of $\mathcal{C}\left(\dot{\Delta}_{n}\right)$ modulo the full subcategory of split diagrams. Restriction induces an equivalence

$$
\underline{\mathcal{C}^{+}\left(\bar{\Delta}_{n}^{\#}\right)} \stackrel{\left.(-)\right|_{\dot{\Delta}_{n}}}{\sim} \underline{\mathcal{C}\left(\dot{\Delta}_{n}\right)}
$$

which is also a useful technical tool; see Proposition 2.6.

At first sight, one might be inclined to prefer $\mathcal{C}\left(\dot{\Delta}_{n}\right)$ over $\mathcal{C}^{+}\left(\bar{\Delta}_{n}^{\#}\right)$. It contains smaller diagrams and has a less elaborate definition. By transport of structure along $(*)$, one obtains an outer shift on $\mathcal{C}\left(\dot{\Delta}_{n}\right)$ as well. By pointwise application of the shift functor on $\mathcal{C}$, we also obtain an inner shift on $\mathcal{C}\left(\dot{\Delta}_{n}\right)$. These could be compared in order to write down a definition of Heller triangulated categories.

So why then did we prefer to use $\mathcal{C}^{+}\left(\bar{\Delta}_{n}^{\#}\right)$ in our definition of Heller triangulated categories in $\S 0.2 .2$ ? Working with $\overline{\mathcal{C}\left(\dot{\Delta}_{n}\right)}$, the indirect definition of the outer shift would cause problems. In practice, one would have to pass the equivalence $(*)$ back and forth. The "blown-up variant" $\mathcal{C}^{+}\left(\bar{\Delta}_{n}^{\#}\right)$ of $\mathcal{C}\left(\dot{\Delta}_{n}\right)$ carries a directly defined outer shift functor and is thus easier to work with. 
There is a further equivalence $\underline{\mathcal{C}\left(\dot{\Delta}_{n}\right)} \stackrel{\sim}{\longrightarrow} \hat{\mathcal{C}}\left(\dot{\Delta}_{n-1}\right)$, where $\hat{\mathcal{C}}$ denotes the Freyd category of $\mathcal{C}$, i.e. the universal abelian category containing $\mathcal{C}$, and where $\underline{\mathcal{C}}\left(\dot{\Delta}_{n-1}\right)$ is the quotient of $\hat{\mathcal{C}}\left(\dot{\Delta}_{n-1}\right)$ modulo split diagrams with entries in $\mathcal{C}$; see Proposition 2.10. Originally HELLER worked with $\underline{\hat{\mathcal{C}}\left(\dot{\Delta}_{n-1}\right)}$ for $n=2$, i.e. with $\hat{\mathcal{C}} / \mathcal{C}$.

\subsection{A result to begin with}

Let $\mathcal{E}$ be a Frobenius category. We define its stable category $\underline{\underline{\mathcal{E}}}$ to be the quotient category of the category of purely acyclic complexes with values in the bijective objects of $\mathcal{E}$, modulo the subcategory of split acyclic such complexes.

Then $\underline{\underline{\mathcal{E}}}$ is equivalent to the classical stable category $\underline{\mathcal{E}}$ of $\mathcal{E}$, defined as the

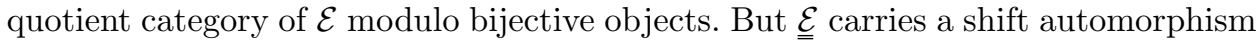
$\mathrm{T}$ (invertible), whereas $\underline{\mathcal{E}}$ carries, in general, only a shift autoequivalence (invertible up to isomorphism). In this sense, $\underline{\underline{\mathcal{E}}}$ is a "strictified version" of $\underline{\mathcal{E}}$.

Theorem (Corollary 4.7, Corollary 4.9). Given a Frobenius category $\mathcal{E}$, there exists a Heller triangulation $\vartheta$ on $(\underline{\mathcal{E}}, \mathrm{T})$. An exact functor $\mathcal{E} \stackrel{E}{\longrightarrow} \mathcal{E}^{\prime}$ between Frobenius categories that sends all bijective objects of $\mathcal{E}$ to bijective objects of $\mathcal{E}^{\prime}$ induces a strictly exact functor $\underline{\underline{\mathcal{E}}} \stackrel{\underline{\underline{E}}}{\longrightarrow} \underline{\underline{\mathcal{E}}}^{\prime}$.

The Verdier triangulated version of this theorem is due to HAPPEL $[\mathbf{1 1}$, Thm. 2.6].

\subsection{A quasicyclic category}

Let $\mathcal{C}$ be a Heller triangulated category.

A quasicyclic category is a contravariant functor from $\bar{\Delta}^{\circ}$ to the (1-)category of categories. Letting qcyc ${ }_{n} \mathcal{C}$ be the subcategory of isomorphisms in $\mathcal{C}^{+}\left(\bar{\Delta}_{n}^{\#}\right)$ for $n \geqslant 0$, we obtain a quasicyclic category qcyc. $\mathcal{C}$. There is a quasicyclic subcategory qcyc ${ }^{\vartheta}=1 \mathcal{C}$ that consists only of $n$-triangles and their isomorphisms instead of all objects in $\mathcal{C}^{+}\left(\bar{\Delta}_{n}^{\#}\right)$ and their isomorphisms. ${ }^{4}$

Restricting qcyc ${ }^{\vartheta=1} \mathcal{C}$ along the functor $\Delta^{\circ} \longleftrightarrow \bar{\Delta}^{\circ}$ of "periodic prolongation", this yields a simplicial category, hence a topological space, depending functorially on $\mathcal{C}$. This space is the author's tentative proposal for the definition of the K-theory of $\mathcal{C}$; cf. [25, Rem. 63]. Of course, this definition still needs to be justified by results one expects of such a K-theory, which has not yet been attempted.

\subsection{Some remarks}

A comparison of our theory to the dérivateur approach and related constructions in [4], [14, chap. V.1], [13], [10], [18], [7] and [22] would be interesting. One might ask whether the base category of a triangulated dérivateur in the sense of [22] carries a Heller triangulation; and if so, whether morphisms of triangulated dérivateurs give rise to strictly exact functors.

Our approach differs from the dérivateur approach in that we consider a single category $\mathcal{C}$ with shift and an "exactness structure", i.e. a Heller triangulation, on it.

\footnotetext{
${ }^{4}$ Here, " $\vartheta=1 "$ is a mere symbol that should evoke the definition of $n$-triangles via $\vartheta$.
} 
The categories $\mathcal{C}^{+}\left(\bar{\Delta}_{n}^{\#}\right)$ needed to define this "exactness structure" on $\mathcal{C}$ consist of veritable $\mathcal{C}$-valued diagrams; see $\S 0.2$. In particular, a "structure preserving map" between two such categories $\mathcal{C}$ and $\mathcal{C}^{\prime}$, i.e. a strictly exact functor, is a single additive functor $\mathcal{C} \stackrel{F}{\longrightarrow} \mathcal{C}^{\prime}$ compatible with the "exactness structures" imposed on $\mathcal{C}$ and on $\mathcal{C}^{\prime}$. In contrast, a "structure preserving map" of triangulated dérivateurs is a compatible family of additive functors.

The generalised triangles in $[3,1.1 .14]$ are, in our language, $n$-pretriangles for which the 2-pretriangle obtained by restriction along any periodic monotone map $\bar{\Delta}_{2} \longrightarrow \bar{\Delta}_{n}$ is a 2-triangle. An $n$-triangle is such a generalised triangle, but the converse does not hold in general, as pointed out to me by A. NEeman. For an example, see $[\mathbf{2 0}, \S 2]$.

Concerning the motivation to consider triangulated categories at all, and in particular derived categories, conceived by GROTHENDIECK, we refer the reader to the introduction of the thesis of VERDIER [28]; see also [15] and [31, p. 26].

\section{Acknowledgements}

I thank A. Wiedemann for an introduction to derived categories. I thank A. Beligiannis for directing me to Heller's parametrisation of Puppe triangulations, and for helpful remarks. I thank B. KELLER for the hint how to "strictify" the classical stable category of a Frobenius category using acyclic complexes. I thank A. NeEman for the hint leading to the folding operation, and for corrections.

More than once I returned to A. HELLER's original construction [12, pp. 53-54], the reference not only for the basic idea, but also for arguments perfectly extendable to the more general framework used here.

\subsection{Notations and conventions}

(i) The disjoint union of sets $X$ and $Y$ is written $X \sqcup Y$.

(ii) Given $a, b, c \in \mathbf{Z}$, the assertion $a \equiv_{c} b$ is defined to hold if there exists a $z \in \mathbf{Z}$ such that $a-b=c z$.

(iii) For $a, b \in \mathbf{Z}$, we denote by $[a, b]:=\{z \in \mathbf{Z}: a \leqslant z \leqslant b\}$ the integral interval. Similarly, we let $[a, b[:=\{z \in \mathbf{Z}: a \leqslant z<b\}] a, b]:,=\{z \in \mathbf{Z}: a<z \leqslant b\}$, $\mathbf{Z}_{\geqslant 0}:=\{z \in \mathbf{Z}: z \geqslant 0\}$ and $\mathbf{Z}_{\leqslant 0}:=\{z \in \mathbf{Z}: z \leqslant 0\}$.

(iv) All categories are supposed to be small with respect to a sufficiently large universe.

(v) Given a category $\mathcal{C}$, and objects $X, Y$ in $\mathcal{C}$, we denote the set of morphisms from $X$ to $Y$ by $c(X, Y)$, or simply by $(X, Y)$, if unambiguous.

(vi) Given a poset $P$, we frequently consider it as a category, letting $P(x, y)$ contain one element $y / x$ if $x \leqslant y$, and letting it be empty if $x \notin y$, where $x, y \in \mathrm{Ob} P=$ $P$.

(vii) Given $n \geqslant 0$, we denote by $\Delta_{n}:=[0, n]$ the linearly ordered set with ordering induced by the standard ordering on $\mathbf{Z}$. Let $\dot{\Delta}_{n}:=\Delta_{n} \backslash\{0\}=[1, n]$, considered as a linearly ordered set. 
(viii) Maps act on the right. Composition of maps, and of more general morphisms, is written on the right, i.e. $\stackrel{a}{\longrightarrow} \stackrel{b}{\longrightarrow}=\stackrel{a b}{\longrightarrow}$.

(ix) Functors act on the right. Composition of functors is written on the right, i.e. $\stackrel{F}{\longrightarrow} \stackrel{G}{\longrightarrow}=\stackrel{F G}{\longrightarrow}$. Accordingly, the entry of a transformation $a$ between functors at an object $X$ will be written $X a$. (The reason for this convention is that we will mainly consider functors of type "restriction to a subdiagram" or "shift", and such operations are usually written on the right.)

(x) A functor is called strictly dense if its map on the objects is surjective. It is called dense if its induced map on the isoclasses is surjective.

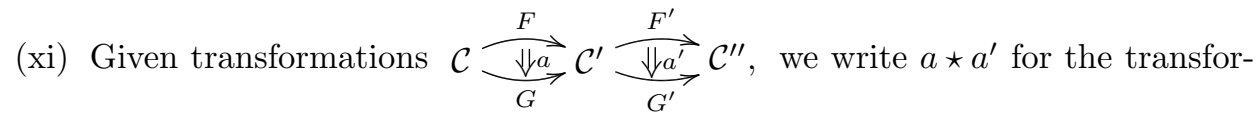
mation from $F F^{\prime}$ to $G G^{\prime}$ given at $X \in \mathrm{ObC}$ by $X\left(a \star a^{\prime}\right):=\left(X F a^{\prime}\right)\left(X a G^{\prime}\right)=$ $\left(X a F^{\prime}\right)\left(X G a^{\prime}\right)$. In this context, we also write the object $F$ for the identity $1_{F}$ on this object, i.e. for example $X\left(F \star a^{\prime}\right)=X\left(1_{F} \star a^{\prime}\right)=(X F) a^{\prime}$.

(xii) The inverse of an isomorphism $f$ is denoted by $f^{-}$. Note that if we denote an iterated shift automorphism $f \longmapsto f^{+1}$ by $f \longmapsto f^{+z}$ for $z \in \mathbf{Z}$, then we have to distinguish between $f^{-}$(inverse isomorphism if $f$ is an isomorphism) and $f^{-1}$ (inverse of the shift functor applied to $f$ ).

(xiii) In an exact category, pure monomorphy is indicated by $X \rightarrow Y$, pure epimorphy by $X \mapsto Y$.

(xiv) A morphism in an additive category $\mathcal{A}$ is split if it is isomorphic, in $\mathcal{A}\left(\Delta_{1}\right)$, to a morphism of the form $X \oplus Y \stackrel{\left(\begin{array}{ll}0 & 0 \\ 1 & 0\end{array}\right)}{\longrightarrow} Y \oplus Z$. A morphism being split is indicated by $X>Y$ (not to be confused with monomorphy). Accordingly, a morphism being a split monomorphism is indicated by $X>\bullet>Y$, a morphism being a split epimorphism by $X>1>Y$. See $\S A .2 .1$.

(xv) We say that idempotents split in an additive category $\mathcal{A}$ if every endomorphism $e$ in $\mathcal{A}$ that satisfies $e^{2}=e$ is split.

(xvi) The category of functors and transformations from a category $D$ to a category $\mathcal{C}$ is denoted by $\llbracket D, \mathcal{C} \rrbracket$ or by $\mathcal{C}(D)$. We also refer to objects in $\mathcal{C}(D)$ as diagrams on $D$ with values or entries in $\mathcal{C}$.

(xvii) If $\mathcal{C}$ and $D$ are categories, and $X \in \mathrm{Ob} \mathcal{C}(D)$, we usually write $(d \stackrel{a}{\longrightarrow} e) X=$ : $\left(X_{d} \stackrel{X_{a}}{\longrightarrow} X_{e}\right)$ for a morphism $d \stackrel{a}{\longrightarrow} e$ in $D$. If the morphism $a$ is unambiguously given by the context, we also use small letters to write $\left(X_{d} \stackrel{x}{\longrightarrow} X_{e}\right):=$ $\left(X_{d} \stackrel{X_{a}}{\longrightarrow} X_{e}\right)$ (similarly $X_{d}^{\prime} \stackrel{x^{\prime}}{\longrightarrow} X_{e}^{\prime}, \tilde{Y}_{d} \stackrel{\tilde{y}}{\longrightarrow} \tilde{Y}_{e}, \ldots$ )

(xviii) Let Add denote the 2-category of additive categories.

(xix) Given an additive category $\mathcal{A}$ and a full additive subcategory $\mathcal{B} \subseteq \mathcal{A}$, we denote by $\mathcal{A} / \mathcal{B}$ the quotient of $\mathcal{A}$ by $\mathcal{B}$, having as objects the objects of $\mathcal{A}$ and as morphisms equivalence classes of morphisms of $\mathcal{A}$. Two morphisms $f$ and $f^{\prime}$ are equivalent in this sense, written $f \equiv_{\mathcal{B}} f^{\prime}$, if their difference factors over an object of $\mathcal{B}$. 
(xx) In an exact category, an object $P$ is called projective if $(P,-)$ turns pure epimorphisms into epimorphisms. An object $I$ is called injective if $(-, I)$ turns pure monomorphisms into epimorphisms. It is called bijective if it is injective and projective. See $\S$ A.2 for details.

(xxi) In an additive category, a morphism $K \stackrel{i}{\longrightarrow} X$ is called a weak kernel of a morphism $X \stackrel{f}{\longrightarrow} Y$ if for every morphism $T \stackrel{t}{\longrightarrow} X$ with $t f=0$ there exists a morphism $T \stackrel{t^{\prime}}{\longrightarrow} K$ with $t^{\prime} i=t$. A weak cokernel is defined dually. An additive category is called weakly abelian if every morphism has a weak kernel and a weak cokernel, and is a weak kernel and a weak cokernel.

(xxii) The Freyd category of a weakly abelian category $\mathcal{C}$ is written $\hat{\mathcal{C}}$. See $\S$ A.6.3 for details.

(xxiii) In an abelian category, a commutative quadrangle

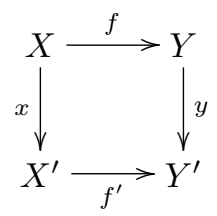

is called a square if its diagonal sequence $X \stackrel{(x f)}{\longrightarrow} X^{\prime} \oplus Y \stackrel{\left(\begin{array}{c}f^{\prime} \\ -y\end{array}\right)}{\longrightarrow} Y^{\prime}$ is short exact. Being a square is indicated by a box sign " $\square$ " in the quadrangle. The quadrangle $\left(X, Y, X^{\prime}, Y^{\prime}\right)$ is called a weak square if its diagonal sequence is exact in the middle; see Definition A.9. Being a weak square is indicated by a "+"-sign in the quadrangle.

In an exact category, $\left(X, Y, X^{\prime}, Y^{\prime}\right)$ is a pure square if it has a pure short exact diagonal sequence. Being a pure square is indicated by a box sign " $\square$ " in the quadrangle.

In a weakly abelian category, $\left(X, Y, X^{\prime}, Y^{\prime}\right)$ is a weak square if it is a weak square in the Freyd category of that weakly abelian category.

(xxiv) In an abelian category, given a morphism $X \stackrel{f}{\longrightarrow} Y$, we sometimes denote its kernel by $\mathrm{K}_{f}$, and its cokernel by $\mathrm{C}_{f}$.

\section{Definition of a Heller triangulated category}

\subsection{Periodic linearly ordered sets and their strips}

Without further comment, we consider a poset $D$ as a category, whose set of objects is given by $D$, and for which $\#_{D}(\alpha, \beta)=1$ if $\alpha \leqslant \beta$, and $\#_{D}(\alpha, \beta)=0$ otherwise. If existent, i.e. if $\alpha \leqslant \beta$, the morphism from $\alpha$ to $\beta$ is denoted by $\beta / \alpha$. A full subposet of a category is a full subcategory that is a poset. In particular, a full subposet of a poset is just a full subcategory of that poset.

A periodic poset is a poset $P$ together with an automorphism T: $P \stackrel{\sim}{\longrightarrow} P$, $\alpha \longmapsto \alpha \mathrm{T}=: \alpha^{+1}$. Likewise, we denote $\alpha \mathrm{T}^{m}=: \alpha^{+m}$ resp. $\alpha \mathrm{T}^{-m}=: \alpha^{-m}$ for $m \in \mathbf{Z}_{\geqslant 0}$. By abuse of notation, we denote a periodic poset $(P, \mathbf{T})$ simply by $P$. 
A morphism of periodic posets $P \stackrel{p}{\stackrel{p}{~}} P^{\prime}$ is a monotone map $p$ of the underlying posets such that $\left(\alpha^{\prime+1}\right) p=\left(\left(\alpha^{\prime}\right) p\right)^{+1}$ for all $\alpha^{\prime} \in P^{\prime}$. The category of periodic posets shall be denoted by $\mathfrak{p p}$.

A periodic linearly ordered set is a periodic poset, the underlying poset of which is linearly ordered, i.e. such that $\#\left({ }_{D}(\alpha, \beta) \cup{ }_{D}(\beta, \alpha)\right)=1$ for all $\alpha, \beta \in D$.

To any linearly ordered set $D$ we can attach a periodic linearly ordered set $\bar{D}$ by letting $\bar{D}:=D \times \mathbf{Z}$, and $(\alpha, z) \leqslant(\beta, w)$ if $z \leqslant w$, or if $(z=w$ and $\alpha \leqslant \beta$ in $D)$. We let $(\alpha, z)^{+1}:=(\alpha, z+1)$. Sending $D \longrightarrow \bar{D}, \alpha \longmapsto(\alpha, 0)$, and identifying $D$ with its image, we obtain $(\alpha, z)=\alpha^{+z}$, and the latter is the notation we will usually use. The periodic linearly ordered set $\bar{D}$ is called the periodic repetition of $D$. Likewise the functor $D \longmapsto \bar{D}$ from the category of linearly ordered sets to the category of periodic linearly ordered sets is called periodic repetition.

Let $\boldsymbol{\Delta}$ be the full subcategory of the category of linearly ordered sets defined by Ob $\boldsymbol{\Delta}:=\left\{\Delta_{n}: n \in \mathbf{Z}_{\geqslant 0}\right\}$.

Let $\bar{\Delta}$ be the full subcategory of the category of periodic linearly ordered sets defined by ${ }^{5}$ Ob $\bar{\Delta}:=\left\{\bar{\Delta}_{n}: n \in \mathbf{Z}_{\geqslant 0}\right\}$.

The reason for considering periodic linearly ordered sets is that the functor periodic repetition from $\boldsymbol{\Delta}$ to $\bar{\Delta}$ is dense and faithful but not full. We will require a naturality of a certain construction with respect to $P \in \mathrm{Ob} \overline{\boldsymbol{\Delta}}$, which is stronger than setting $P=\bar{D}$ and requiring naturality with respect to $D \in \mathrm{Ob} \boldsymbol{\Delta}$.

Given $n \geqslant 0$, the underlying linearly ordered set of $\bar{\Delta}_{n}$ is isomorphic to $\mathbf{Z}$ via $\alpha^{+z} \longmapsto \alpha+(n+1) z$. We use this isomorphism to define the operation

$$
\bar{\Delta}_{n} \times \mathbf{Z} \longrightarrow \bar{\Delta}_{n},\left(\alpha^{+z}, x\right) \longmapsto \alpha^{+z}+x:=(\overline{\alpha+x})^{+(z+\underline{\alpha+x})}
$$

where we write $k=(n+1) \underline{k}+\bar{k}$ with $\underline{k} \in \mathbf{Z}$ and $\bar{k} \in[0, n]$ for $k \in \mathbf{Z}$. For instance, if $n=3$, then $2^{+1}+7=1^{+3}$.

To a periodic linearly ordered set $P$, we attach the poset

$$
P^{\#}:=\left\{\beta / \alpha \in P\left(\Delta_{1}\right): \beta^{-1} \leqslant \alpha \leqslant \beta \leqslant \alpha^{+1}\right\}
$$

as a full subposet of $P\left(\Delta_{1}\right)$, called the strip of $P$. A morphism therein from $\beta / \alpha$ to $\delta / \gamma$ is written $\delta / \gamma / / \beta / \alpha$, which is unique if it exists, i.e. if $\alpha \leqslant \gamma$ and $\beta \leqslant \delta$.

The strip $P^{\#}$ carries the automorphism $\beta / \alpha \longmapsto(\beta / \alpha)^{+1}:=\alpha^{+1} / \beta$ in $\mathfrak{p p}$, where $\beta / \alpha \in P^{\#}$. If $P=\bar{\Delta}_{n}$, we also write $\beta / \alpha \stackrel{\mathrm{T}_{n}}{\longmapsto}(\beta / \alpha)^{+1}$.

This construction defines a functor

$$
\begin{aligned}
\bar{\Delta} \stackrel{(-)^{\#}}{\longrightarrow} \mathfrak{p p} \\
P \longmapsto P^{\#}
\end{aligned}
$$

which sends a morphism $P \stackrel{p}{\longleftarrow} P^{\prime}$ in $\bar{\Delta}$ to

$$
\begin{gathered}
P^{\#} \stackrel{p^{\#}}{\longleftarrow} P^{\prime \#} \\
\beta^{\prime} p / \alpha^{\prime} p \longleftarrow \beta^{\prime} / \alpha^{\prime}
\end{gathered}
$$

In fact, $p^{\#}$ is well defined, since if $\beta^{\prime-1} \leqslant \alpha^{\prime} \leqslant \beta^{\prime} \leqslant \alpha^{\prime+1}$, then $\left(\beta^{\prime} p\right)^{-1} \leqslant \alpha^{\prime} p \leqslant$ $\beta^{\prime} p \leqslant\left(\alpha^{\prime} p\right)^{+1}$. Moreover, $p^{\#}$ is monotone and compatible with shift.

\footnotetext{
${ }^{5}$ The category $\bar{\Delta}$ is isomorphic to the category $L$ defined by ELMENDORF in [6].
} 
Example 1.1. The periodic poset $\bar{\Delta}_{2}^{\#}$, i.e. the strip of the periodic repetition of $\Delta_{2}$, can be displayed as

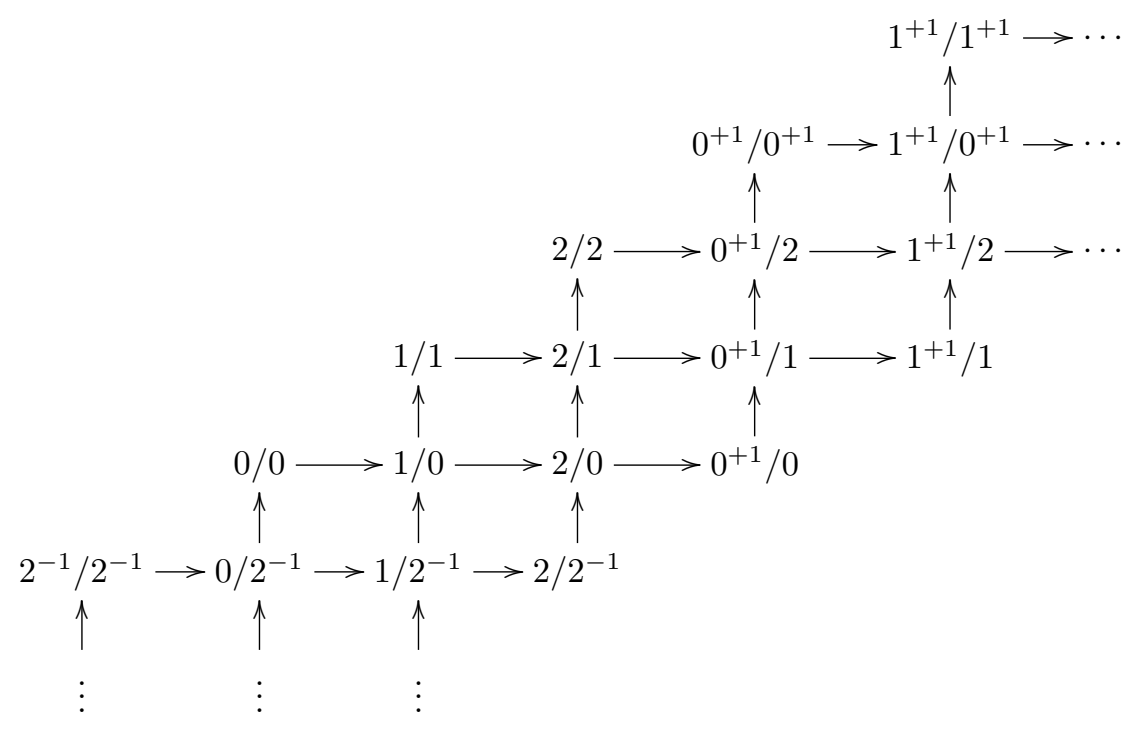

\subsection{Heller triangulated categories}

Suppose given a weakly abelian category $\mathcal{C}$; cf. Definition A.26. From $\S 1.2 .1 .3$ on, we assume it to be equipped with an automorphism

$$
\begin{aligned}
\mathcal{C} & \stackrel{\mathrm{T}}{\sim} \mathcal{C} \\
(X \stackrel{u}{\longrightarrow} Y) & \longmapsto(X \mathrm{~T} \stackrel{u \mathrm{~T}}{\longrightarrow} Y \mathrm{~T})=:\left(X^{+1} \stackrel{u^{+1}}{\longrightarrow} Y^{+1}\right) .
\end{aligned}
$$

Similarly, we denote $\left(X \mathrm{~T}^{m} \stackrel{u \mathrm{~T}^{m}}{\longrightarrow} Y \mathrm{~T}^{m}\right)=:\left(X^{+m} \stackrel{u^{+m}}{\longrightarrow} Y^{+m}\right)$ for $m \in \mathbf{Z}$.

Recall that its Freyd category $\hat{\mathcal{C}}$ is an abelian Frobenius category, and that the image of $\mathcal{C}$ in $\hat{\mathcal{C}}$, identified with $\mathcal{C}$, is a sufficiently large subcategory of bijectives; see $\S$ A.6.3.

\subsubsection{The Stable Category of Pretriangles $\mathcal{C}^{+}\left(P^{\#}\right)$}

\subsubsection{Definition of $\mathcal{C}^{+}\left(P^{\#}\right)$}

Concerning the Freyd category $\hat{\mathcal{C}}$ of $\mathcal{C}$, see $\S$ A.6.3. Concerning the notion of a weak square in $\hat{\mathcal{C}}$, see Definition A.9. A weak square in $\mathcal{C}$ is a weak square in $\hat{\mathcal{C}}$ that has all four objects in $\mathrm{ObC}$. Applying Remark A.27, we obtain an elementary way to characterise weak squares as having a diagonal sequence with first morphism being a weak kernel of the second; or, equivalently, with second morphism being a weak cokernel of the first.

Given a periodic linearly ordered set $P$, we let $\mathcal{C}^{+}\left(P^{\#}\right)$ be the full subcategory 
of $\mathcal{C}\left(P^{\#}\right)$ defined by

$$
\mathrm{ObC}^{+}\left(P^{\#}\right):=\left\{\begin{array}{l}
X \in \mathrm{Ob} \mathcal{C}\left(P^{\#}\right): \\
\end{array}\right.
$$

1) $X_{\alpha / \alpha}=0$ and $X_{\alpha+1 / \alpha}=0$

for all $\alpha \in P$.

2) For all $\delta^{-1} \leqslant \alpha \leqslant \beta \leqslant \gamma \leqslant \delta \leqslant \alpha^{+1}$ in $P$, the quadrangle

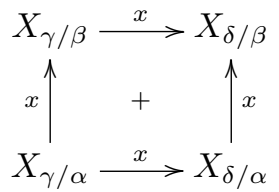

is a weak square (as indicated by +$)$.

Note that we do not require that $\left(X_{\alpha^{+1} / \gamma} \stackrel{x}{\longrightarrow} X_{\beta^{+1} / \delta}\right)=\left(X_{\gamma / \alpha} \stackrel{x}{\longrightarrow} X_{\delta / \beta}\right)^{+1}$ for $\gamma / \alpha, \delta / \beta \in P^{\#}$ with $\gamma / \alpha \leqslant \delta / \beta$.

An object of $\mathcal{C}^{+}\left(P^{\#}\right)$ is called a $\boldsymbol{P}$-pretriangle. Given $n \geqslant 0$, an object of $\mathcal{C}^{+}\left(\bar{\Delta}_{n}^{\#}\right)$, i.e. a $\bar{\Delta}_{n}$-pretriangle, is also called an $n$-pretriangle.

Roughly put, an $n$-pretriangle is a diagram on the strip $\bar{\Delta}_{n}^{\#}$ of the periodic repetition $\bar{\Delta}_{n}$ of $\Delta_{n}$ consisting of weak squares with zeroes on the boundaries.

Example 1.2. A 0-pretriangle consists of zero objects. A 1-pretriangle is just a sequence $\ldots, X_{0 / 1^{-1}}, X_{1 / 0}, X_{0^{+1} / 1}, \ldots$ of objects of $\mathcal{C}$, decorated with some zero objects. A 2-pretriangle is a complex in $\mathcal{C}$ which becomes acyclic in $\hat{\mathcal{C}}$ - for short, which is acyclic - decorated with some zero objects.

A morphism in $\mathcal{C}$ is called split if it factors in $\mathcal{C}$ into a retraction followed by a coretraction. Equivalently, its image, taken in $\hat{\mathcal{C}}$, is bijective as an object of $\hat{\mathcal{C}}$.

Let $\mathcal{C}^{+, \text {split }}\left(P^{\#}\right)$ be the full subcategory of $\mathcal{C}^{+}\left(P^{\#}\right)$ defined by

$$
\operatorname{ObC}^{+, \text {split }}\left(P^{\#}\right):=\left\{X \in \mathrm{ObC}^{+}\left(P^{\#}\right): \begin{array}{l}
X_{\gamma / \alpha} \underset{x}{ } X_{\delta / \beta} \text { is split } \\
\text { with } \gamma / \alpha \leqslant \delta / \beta
\end{array}\right\} .
$$

We denote the quotient category by

$$
\underline{\mathcal{C}^{+}\left(P^{\#}\right)}:=\mathcal{C}^{+}\left(P^{\#}\right) / \mathcal{C}^{+, \text {split }}\left(P^{\#}\right),
$$

called the stable category of $\boldsymbol{P}$-pretriangles.

Example 1.3. We have $\underline{\mathcal{C}^{+}\left(\bar{\Delta}_{0}^{\#}\right)}=\underline{\mathcal{C}^{+}\left(\bar{\Delta}_{1}^{\#}\right)}=0$. The category $\underline{\mathcal{C}^{+}\left(\bar{\Delta}_{2}^{\#}\right)}$ can be regarded as the homotopy category of the category of acyclic complexes with entries in $\mathcal{C}$.

\subsubsection{Naturality of $\underline{\mathcal{C}^{+}\left(P^{\#}\right)}$ in $P$}

Suppose given periodic linearly ordered sets $P, P^{\prime}$, and a morphism $P^{\#} \stackrel{q}{\longleftarrow} P^{\prime \#}$ of periodic posets such that either $\left(P=P^{\prime}\right.$ and $q=\mathrm{T}$, the shift functor on $\left.P^{\#}\right)$ or $q=p^{\#}$ for some morphism $P \stackrel{p}{\longleftarrow} P^{\prime}$ of periodic linearly ordered sets. Recall that 
if $P=\bar{\Delta}_{n}$, then we alternatively write $\mathrm{T}_{n}$ for the shift functor $\mathrm{T}$ on $\bar{\Delta}_{n}^{\#}$. We obtain an induced functor

$$
\begin{aligned}
\mathcal{C}^{+}\left(P^{\#}\right) & \stackrel{\mathcal{C}^{+}(q)}{\longrightarrow} \mathcal{C}^{+}\left(P^{\prime \#}\right) \\
X & \longmapsto X \mathcal{C}^{+}(q):=q X,
\end{aligned}
$$

given by composition of $q$, followed by $X$. In particular, the shift T on $P^{\#}$ induces a functor

$$
\begin{aligned}
\mathcal{C}^{+}\left(P^{\#}\right) & \stackrel{\mathcal{C}^{+}(\mathrm{T})}{\longrightarrow} \mathcal{C}^{+}\left(P^{\#}\right) \\
X & \longmapsto[X]^{+1}:=X\left(\mathcal{C}^{+}(\mathrm{T})\right),
\end{aligned}
$$

called the outer shift. Note that if $P=\bar{\Delta}_{n}$, then $[X]_{\beta / \alpha}^{+1}=X_{(\beta / \alpha)^{+1}}=X_{\alpha^{+1} / \beta}$ for $\beta / \alpha \in \bar{\Delta}_{n}^{\#}$.

On the stable category, this functor induces a functor

$$
\begin{aligned}
& \underline{\mathcal{C}^{+}\left(P^{\#}\right)} \stackrel{\mathcal{C}^{+}(\mathrm{T})}{\longrightarrow} \underline{\mathcal{C}^{+}\left(P^{\#}\right)} \\
& X \longmapsto[X]^{+1},
\end{aligned}
$$

likewise called the outer shift.

Given a morphism $P \stackrel{p}{\longleftarrow} P^{\prime}$ in $\bar{\Delta}$, we obtain an induced morphism $P^{\#} \stackrel{p^{\#}}{\longleftarrow} P^{\prime \#}$, and hence an induced functor usually abbreviated by

$$
\begin{aligned}
& \mathcal{C}^{+}\left(P^{\#}\right) \stackrel{p^{\#}:=\mathcal{C}^{+}\left(p^{\#}\right)}{\longrightarrow} \mathcal{C}^{+}\left(P^{\prime \#}\right) \\
& X \longmapsto X p^{\#}:=X\left(\mathcal{C}^{+}\left(p^{\#}\right)\right) .
\end{aligned}
$$

Likewise on the stable categories; we abbreviate $\underline{p}^{\#}:=\mathcal{C}^{+}\left(p^{\#}\right)$.

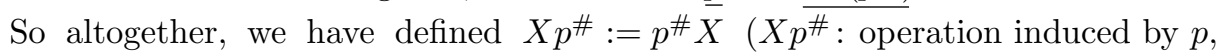
applied to $X ; p^{\#} X$ : composition of $p^{\#}$ and $X$ ), which is a bit unfortunate, but convenient in practice.

Given a morphism $P \stackrel{p}{\longleftarrow} P^{\prime}$ in $\overline{\boldsymbol{\Delta}}$ and $X \in \mathrm{ObC}^{+}\left(P^{\#}\right)$, we have

$$
[X]^{+1} p^{\#}=\left[X p^{\#}\right]^{+1}
$$

natural in $X$. Likewise on the stable categories. if

Given $P, P^{\prime} \in \mathrm{Ob} \overline{\boldsymbol{\Delta}}$, a functor $\mathcal{C}^{+}\left(P^{\#}\right) \stackrel{F}{\longrightarrow} \mathcal{C}^{+}\left(P^{\prime \#}\right)$ is called strictly periodic

$$
[X F]^{+1}=[X]^{+1} F
$$

natural in $X$. Likewise on the stable categories.

\subsubsection{Naturality of $\mathcal{C}^{+}\left(P^{\#}\right)$ in $\mathcal{C}$}

An additive functor $\mathcal{C} \stackrel{F}{\longrightarrow} \mathcal{C}^{\prime}$ is called subexact if the induced additive functor $\hat{\mathcal{C}} \stackrel{\hat{F}}{\longrightarrow} \hat{\mathcal{C}}^{\prime}$ is an exact functor of abelian categories; see $\S$ A.6.3. Alternatively, it is subexact if and only if it preserves weak kernels, or, equivalently, weak cokernels; see Remark A.27. 
Homology, Homotopy and Applications, vol. 9(2), 2007

Suppose given a subexact functor $\mathcal{C} \stackrel{F}{\longrightarrow} \mathcal{C}^{\prime}$ and $P \in \mathrm{Ob} \boldsymbol{\Delta}$. We obtain an induced functor

$$
\begin{aligned}
\mathcal{C}^{+}\left(P^{\#}\right) & \stackrel{F^{+}\left(P^{\#}\right)}{\longrightarrow} \mathcal{C}^{\prime+}\left(P^{\#}\right) \\
X & \longmapsto X F^{+}\left(P^{\#}\right),
\end{aligned}
$$

where, writing $Y:=X F^{+}\left(P^{\#}\right)$, we let

$$
\left(Y_{\beta / \alpha} \stackrel{y}{\longrightarrow} Y_{\delta / \gamma}\right):=\left(X_{\beta / \alpha} F \stackrel{x F}{\longrightarrow} X_{\delta / \gamma} F\right)
$$

for $\beta / \alpha, \delta / \gamma \in P^{\#}$ with $\beta / \alpha \leqslant \delta / \gamma$.

In particular, the automorphism $\mathcal{C} \stackrel{\mathrm{T}}{\longrightarrow} \mathcal{C}$ induces an automorphism

$$
\begin{aligned}
\mathcal{C}^{+}\left(P^{\#}\right) & \stackrel{\mathrm{T}^{+}\left(P^{\#}\right)}{\longrightarrow} \mathcal{C}^{+}\left(P^{\#}\right) \\
X & \longmapsto\left[X^{+1}\right]:=X\left(\mathrm{~T}^{+}\left(P^{\#}\right)\right),
\end{aligned}
$$

called the inner shift. Note that if $P=\bar{\Delta}_{n}$, then $\left[X^{+1}\right]_{\beta / \alpha}=X_{\beta / \alpha}^{+1}$ for $\beta / \alpha \in \bar{\Delta}_{n}^{\#}$.

On the stable category, this induces an automorphism

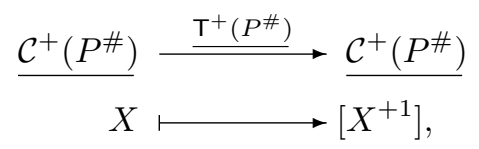

likewise called the inner shift.

\subsubsection{FOLDING}

The following construction arose from a hint of A. NEEMAN, who showed me a multitude of 2-triangles in an $n$-triangle similar to the two 2-triangles explained in $[3,1.1 .13]$; cf. Definition 1.5.(ii) below.

\subsubsection{SOME NOTATION}

Given $P=(P, \mathrm{~T}) \in \mathrm{Ob} \overline{\boldsymbol{\Delta}}$, we denote by $2 P$ the periodic poset $\left(P, \mathrm{~T}^{2}\right)$.

Given a linearly ordered set $D$, we let $\rho \sqcup D$ be the linearly ordered set having as underlying set $\{\rho\} \sqcup D$; and as partial order $\rho \leqslant \rho \sqcup D \alpha$ for all $\alpha \in D$, and $\alpha \leqslant \rho \sqcup D \beta$ if $\alpha, \beta \in D$ and $\alpha \leqslant_{D} \beta$.

Roughly put, $2 P$ is $P$ with doubled period, and $\rho \sqcup D$ is $D$ with an added initial object $\rho$.

Let $n \geqslant 0$. We have an isomorphism of periodic linearly ordered sets

$$
\begin{aligned}
2 \bar{\Delta}_{n} & \sim \bar{\Delta}_{2 n+1} \\
k^{+l} & \longmapsto \begin{cases}k^{+l / 2} & \text { for } l \equiv_{2} 0 \\
(k+n+1)^{+(l-1) / 2} & \text { for } l \equiv_{2} 1\end{cases}
\end{aligned}
$$

and an isomorphism of linearly ordered sets

$$
\begin{aligned}
\rho \sqcup \Delta_{n} & \stackrel{\sim}{\longrightarrow} \Delta_{n+1} \\
k & \longmapsto k+1 \quad \text { for } k \in[0, n] \\
\rho & \longmapsto 0 .
\end{aligned}
$$


In order to remain inside $\overline{\boldsymbol{\Delta}}$ resp. inside $\boldsymbol{\Delta}$, we use these isomorphisms as identifications.

Then $P \longmapsto 2 P$ is natural in $P$ and therefore defines an endofunctor of $\overline{\boldsymbol{\Delta}}$, and $D \longmapsto \rho \sqcup D$ is natural in $D$ and therefore defines an endofunctor of $\boldsymbol{\Delta}$.

Given a linearly ordered set $D$, we will need to consider the periodic posets $2 \bar{D}$ and $\overline{\rho \sqcup D}$, formed using periodic repetition.

\subsubsection{THE FOLDING OPERATION}

Let $n \geqslant 0$. Let the strictly periodic functor

$$
\begin{aligned}
\mathcal{C}^{+}\left(\left(2 \bar{\Delta}_{n}\right)^{\#}\right) & \stackrel{\mathfrak{f}_{n}}{\longrightarrow} \mathcal{C}^{+}\left(\overline{\rho \sqcup \Delta_{n}}{ }^{\#}\right) \\
X & \longmapsto X \mathfrak{f}_{n}
\end{aligned}
$$

be determined on objects by the following data. Writing $Y=X \mathfrak{f}_{n}$, we let

$$
\begin{aligned}
\left(Y_{\alpha / \rho} \stackrel{y}{\longrightarrow} Y_{\beta / \rho}\right) & :=\left(X_{\alpha^{+1} / \alpha} \stackrel{x}{\longrightarrow} X_{\beta^{+1} / \beta}\right) \\
\left(Y_{\beta / \rho} \stackrel{y}{\longrightarrow} Y_{\beta / \alpha}\right): & =\left(X_{\beta+1} / \beta \stackrel{(x x)}{\longrightarrow} X_{\beta^{+1} / \alpha+1} \oplus X_{\alpha+\beta} / \beta\right) \\
\left(Y_{\beta / \alpha} \stackrel{y}{\longrightarrow} Y_{\delta / \gamma}\right): & =\left(X_{\beta^{+1} / \alpha^{+1}} \oplus X_{\alpha^{+2} / \beta} \stackrel{\left(\begin{array}{ll}
x & 0 \\
0 & x
\end{array}\right)}{\longrightarrow} X_{\delta^{+1} / \gamma^{+1}} \oplus X_{\gamma^{+2} / \delta}\right) \\
\left(Y_{\delta / \gamma} \stackrel{y}{\longrightarrow} Y_{\rho^{+1} / \gamma}\right) & :=\left(X_{\delta^{+1} / \gamma^{+1}} \oplus X_{\gamma^{+2} / \delta} \stackrel{\left(\begin{array}{c}
x \\
-x
\end{array}\right)}{\longrightarrow} X_{\gamma^{+2} / \gamma^{+1}}\right)
\end{aligned}
$$

for $\alpha, \beta, \gamma, \delta \in \Delta_{n}$ with $\alpha \leqslant \beta$, with $\gamma \leqslant \delta$ and with $\beta / \alpha \leqslant \delta / \gamma$. The remaining morphisms are given by composition.

Note that $X \in \mathrm{ObC}^{+}\left(\left(2 \bar{\Delta}_{n}\right)^{\#}\right)$, so, for example, $X_{\beta^{+1} / \beta} \neq 0$ is possible, whereas $X_{\beta+2 / \beta}=0$ for $\beta \in \Delta_{n}$.

We claim that $X \mathfrak{f}_{n}$ is an object of $\mathcal{C}^{+}\left(\overline{\rho \sqcup \Delta_{n}}{ }^{\#}\right)$.

In fact, by Lemma A.14, applied in the abelian category $\hat{\mathcal{C}}$, we are reduced to considering the quadrangles of $Y$ on $(\gamma / \rho, \delta / \rho, \gamma / \beta, \delta / \beta)$ for $\beta, \gamma, \delta \in \Delta_{n}$ with $\beta \leqslant \gamma \leqslant \delta$; on $(\gamma / \alpha, \delta / \alpha, \gamma / \beta, \delta / \beta)$ for $\alpha, \beta, \gamma, \delta \in \Delta_{n}$ with $\alpha \leqslant \beta \leqslant \gamma \leqslant \delta$; and on $\left(\gamma / \alpha, \rho^{+1} / \alpha, \gamma / \beta, \rho^{+1} / \beta\right)$ for $\alpha, \beta, \gamma \in \Delta_{n}$ with $\alpha \leqslant \beta \leqslant \gamma$.

The quadrangle of $Y$ on $(\gamma / \alpha, \delta / \alpha, \gamma / \beta, \delta / \beta)$ is a weak square as the direct sum of two weak squares.

For the remaining quadrangles to be treated, Lemma A.17 reduces us to considering the quadrangles of $Y$ on $(\alpha / \rho, \beta / \rho, \alpha / \alpha, \beta / \alpha)$, on $\left(\beta / \rho, \rho^{+1} / \rho, \beta / \alpha, \rho^{+1} / \alpha\right)$ and on $\left(\beta / \alpha, \rho^{+1} / \alpha, \beta / \beta, \rho^{+1} / \beta\right)$ for $\alpha, \beta \in \Delta_{n}$ with $\alpha \leqslant \beta$. These are in fact weak squares, as follows from Lemma A.18 and its dual assertion. This proves our claim.

This construction of $Y=X \mathfrak{f}_{n}$ is functorial in $X$.

To prove that the folding operation passes to the stable categories, we have to show that for an object $X$ of $\mathcal{C}^{+, \text {split }}\left(\left(2 \bar{\Delta}_{n}\right)^{\#}\right)$, the folded object $X \mathfrak{f}_{n}$ is in $\mathcal{C}^{+, \text {split }}\left(\overline{\rho \sqcup \Delta_{n}}{ }^{\#}\right)$. Denote $Y:=X \mathfrak{f}_{n}$. Since $Y_{\alpha / \rho} \stackrel{y}{\longrightarrow} Y_{\beta / \rho}$ is split for all $\alpha, \beta \in \Delta_{n}$ with $\alpha \leqslant \beta$, it suffices to prove the following lemma.

Lemma 1.4. Suppose given $m \geqslant 0$ and $Z \in \mathrm{ObC}^{+}\left(\bar{\Delta}_{m}^{\#}\right)$ such that $Z_{\alpha / 0} \stackrel{z}{\longrightarrow} Z_{\beta / 0}$ is split for all $\alpha, \beta \in \Delta_{m}$ with $0<\alpha \leqslant \beta$. Then $Z \in \operatorname{Ob} \mathcal{C}^{+, \text {split }}\left(\bar{\Delta}_{m}^{\#}\right)$. 
Proof. Consider the morphism $Z_{\gamma / \alpha} \stackrel{z}{\longrightarrow} Z_{\delta / \beta}$ for $\gamma / \alpha \leqslant \delta / \beta$ in $\bar{\Delta}_{m}^{\#}$. We have to show that it is split, i.e. that its image, taken in $\hat{\mathcal{C}}$, is bijective there. Unless $\alpha \leqslant$ $\beta \leqslant \gamma \leqslant \delta \leqslant \alpha^{+1}$, this morphism is zero, hence split. If this condition holds, it is the diagonal morphism of the weak square $\left(Z_{\gamma / \alpha}, Z_{\delta / \alpha}, Z_{\gamma / \beta}, Z_{\delta / \beta}\right)$.

So by Lemma A.19, applied in the abelian category $\hat{\mathcal{C}}$, we see that it suffices to show that the (horizontal) morphism $Z_{\beta / \alpha} \stackrel{z}{\longrightarrow} Z_{\gamma / \alpha}$ is split and that the (vertical) morphism $Z_{\gamma / \alpha} \stackrel{z}{\longrightarrow} Z_{\gamma / \beta}$ is split for all $\alpha, \beta, \gamma$ in $\bar{\Delta}_{m}$ with $\gamma^{-1} \leqslant \alpha \leqslant \beta \leqslant \gamma \leqslant \alpha^{+1}$.

The long exact sequence

$$
\begin{aligned}
\cdots \longrightarrow Z_{\alpha / \beta^{-1}} \longrightarrow Z_{\alpha / \gamma^{-1}} \longrightarrow Z_{\beta / \gamma^{-1}} \longrightarrow \\
\quad Z_{\beta / \alpha} \longrightarrow Z_{\gamma / \alpha} \longrightarrow Z_{\gamma / \beta} \longrightarrow Z_{\alpha^{+1} / \beta} \longrightarrow \cdots
\end{aligned}
$$

in $\hat{\mathcal{C}}$ shows that it suffices to show that the morphism $Z_{\beta / \alpha} \stackrel{z}{\longrightarrow} Z_{\gamma / \alpha}$ is split for all $0 \leqslant \alpha \leqslant \beta \leqslant \gamma<0^{+1}$. In fact, first of all we may assume that $0 \leqslant \alpha<0^{+1}$, so that $0 \leqslant \alpha \leqslant \beta \leqslant \gamma \leqslant \alpha^{+1}<0^{+2}$. Hence either $0 \leqslant \alpha \leqslant \beta \leqslant \gamma<0^{+1}$, or $0 \leqslant \gamma^{-1} \leqslant \alpha \leqslant$ $\beta<0^{+1}$, or $0 \leqslant \beta^{-1} \leqslant \gamma^{-1} \leqslant \alpha<0^{+1}$.

Now we may assume that $0<\alpha$ and apply Lemma A.19 to the weak square $\left(Z_{\beta / 0}, Z_{\gamma / 0}, Z_{\beta / \alpha}, Z_{\gamma / \alpha}\right)$, in which $Z_{\beta / 0} \stackrel{z}{\longrightarrow} Z_{\gamma / 0}$ is split by assumption, in which $Z_{\beta / 0} \stackrel{z}{\longrightarrow} Z_{\beta / \alpha}$ is split since $Z_{\alpha / 0} \stackrel{z}{\longrightarrow} Z_{\beta / 0}$ is split by assumption, and in which $Z_{\gamma / 0} \stackrel{z}{\longrightarrow} Z_{\gamma / \alpha}$ is split since $Z_{\alpha / 0} \stackrel{z}{\longrightarrow} Z_{\gamma / 0}$ is split by assumption.

So the folding operation passes to an operation

$$
\begin{aligned}
\frac{\mathcal{C}^{+}\left(\left(2 \bar{\Delta}_{n}\right)^{\#}\right)}{\underline{\mathfrak{f}}_{n}} & \stackrel{\mathcal{C}^{+}\left(\overline{\rho \sqcup \Delta_{n}}{ }^{\#}\right)}{X} \longmapsto \underline{\underline{f}}_{n}
\end{aligned}
$$

on the stable categories.

\subsubsection{AN EXAMPle: FOlding From $\bar{\Delta}_{5}^{\#}$ TO $\bar{\Delta}_{3}^{\#}$}

Let $D=\Delta_{2}$. Note that $2 \bar{\Delta}_{2} \simeq \bar{\Delta}_{5}$. Let $X \in \mathrm{ObC}^{+}\left(\left(2 \bar{\Delta}_{2}\right)^{\#}\right)$, as depicted in Figure 1.1 .

Note that $\rho \sqcup \Delta_{2} \simeq \Delta_{3}$. Folding turns $X$ into $X \mathfrak{f}_{2} \in \mathrm{ObC}^{+}\left({\overline{\rho \sqcup \Delta_{2}}}^{\#}\right)$, as depicted in Figure 1.2.

\subsubsection{A DEFinition of HELler triangulated CATEgories AND STRICTLY EXACT FUNCTORS}

Recall that $\mathcal{C}$ is a weakly abelian category, and that $T=(-)^{+1}$ is an automorphism of $\mathcal{C}$; cf. Def. A.26. Suppose given $n \geqslant 0$. We have introduced the automorphisms

$$
\begin{aligned}
& \left.\underline{\mathcal{C}^{+}\left(\bar{\Delta}_{n}^{\#}\right)} \stackrel{\stackrel{\mathcal{C}^{+}\left(\mathrm{T}_{n}\right)}{\sim}}{\sim} \underline{\mathcal{C}^{+}\left(\bar{\Delta}_{n}^{\#}\right)} \quad \text { (outer shift; } \S 1.2 .1 .2\right) \\
& X \longmapsto[X]^{+1} \\
& \underline{\mathcal{C}^{+}\left(\bar{\Delta}_{n}^{\#}\right)} \stackrel{\mathrm{T}^{+}\left(\bar{\Delta}_{n}^{\#}\right)}{\sim} \underline{\mathcal{C}^{+}\left(\bar{\Delta}_{n}^{\#}\right)} \quad \text { (inner shift; §1.2.1.3) } \\
& X \longmapsto\left[X^{+1}\right] \text {. }
\end{aligned}
$$




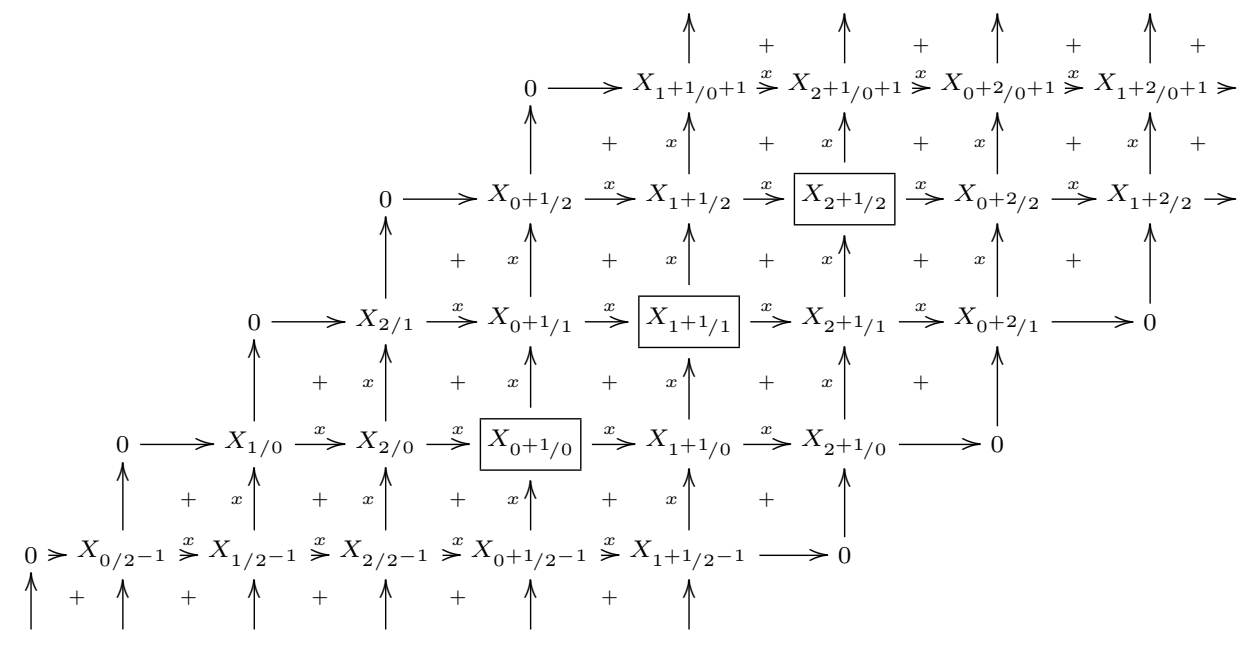

Figure 1.1

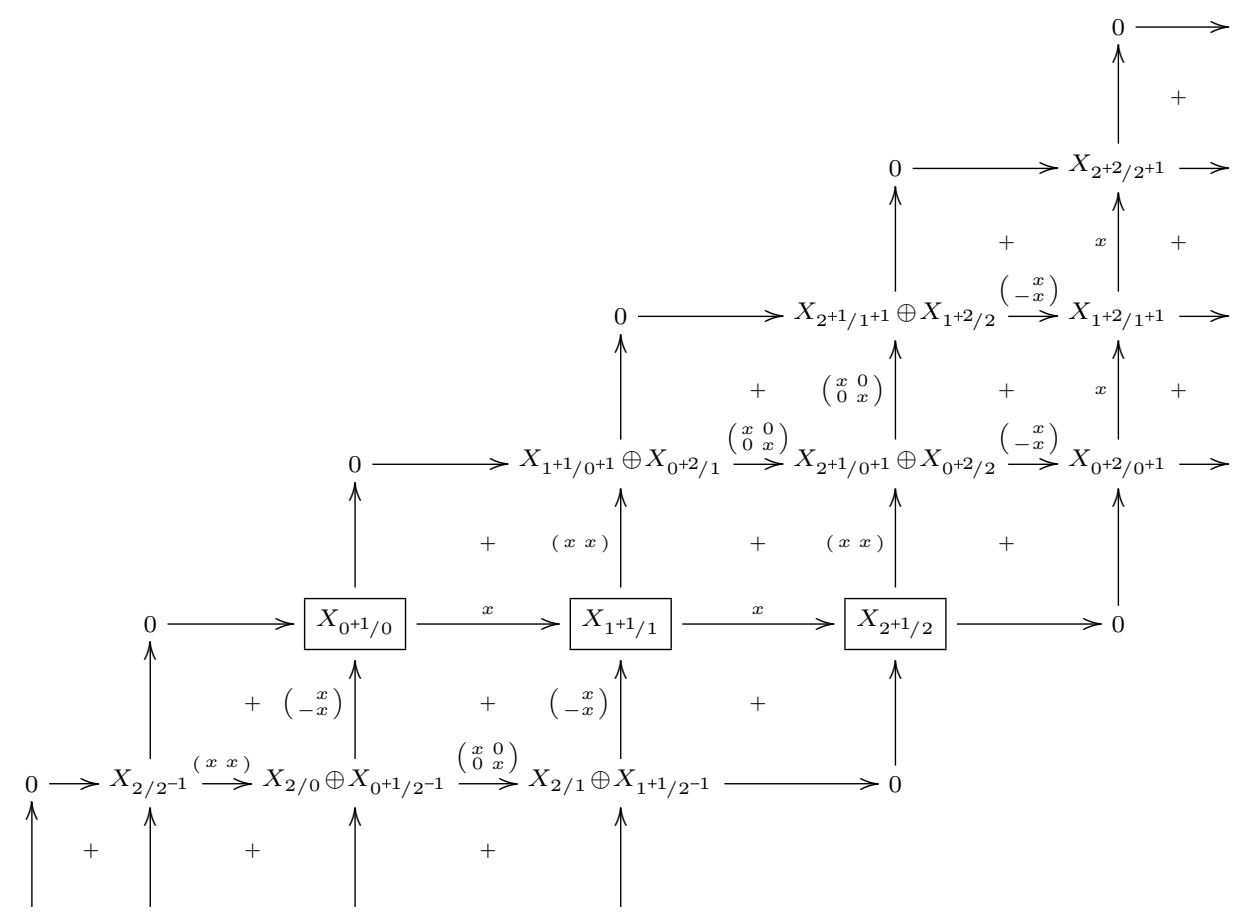

Figure 1.2 
The outer shift shifts the whole diagram $X \in \mathrm{ObC}^{+}\left(\bar{\Delta}_{n}^{\#}\right)$ one step downwards the object $X_{\alpha^{+1} / \beta}$ is the entry of $[X]^{+1}$ at position $\beta / \alpha$.

The inner shift applies the given shift automorphism $(-)^{+1}$ of $\mathcal{C}$ entrywise to a diagram $X \in \mathrm{ObC}^{+}\left(\bar{\Delta}_{n}^{\#}\right)$.

Furthermore, we write $\left[X^{+a}\right]^{+b}:=X \mathrm{~T}^{+}\left(\bar{\Delta}_{n}^{\#}\right)^{a} \mathcal{C}^{+}\left(\mathrm{T}_{n}\right)^{b}=X \mathcal{C}^{+}\left(\mathrm{T}_{n}\right)^{b} \mathrm{~T}^{+}\left(\bar{\Delta}_{n}^{\#}\right)^{a}$ for $a, b \in \mathbf{Z}_{\geqslant 0}, X \in \mathrm{ObC}^{+}\left(\bar{\Delta}_{n}^{\#}\right)$; similarly for $a, b \in \mathbf{Z}$. Likewise in the stable case.

\section{Definition 1.5.}

(i) A Heller triangulation on $(\mathcal{C}, T)$ is a tuple of isomorphisms of functors

$$
\vartheta=\left(\underline{\mathcal{C}^{+}\left(\mathrm{T}_{n}\right)} \stackrel{\vartheta_{n}}{\sim} \underline{\mathrm{T}^{+}\left(\bar{\Delta}_{n}^{\#}\right)}\right)_{n \geqslant 0}=\left([-]^{+1} \stackrel{\vartheta_{n}}{\sim}\left[-^{+1}\right]\right)_{n \geqslant 0}
$$

such that

$$
\underline{p}^{\#} \star \vartheta_{m}=\vartheta_{n} \star \underline{p}^{\#}
$$

for all $n, m \geqslant 0$ and all periodic monotone maps $\bar{\Delta}_{n} \stackrel{p}{\longleftarrow} \bar{\Delta}_{m}$ in $\bar{\Delta}$, and such that

$$
\underline{\mathfrak{f}}_{n} \star \vartheta_{n+1}=\vartheta_{2 n+1} \star \underline{\mathfrak{f}}_{n}
$$

for all $n \geqslant 0$.

(ii) Given a Heller triangulation $\vartheta$ on $(\mathcal{C}, T)$, we use the following terminology.

(1) The triple $(\mathcal{C}, T, \vartheta)$ forms a Heller triangulated category, usually just denoted by $\mathcal{C}$.

(2) Given $n \geqslant 0$, an $n$-triangle is an object $X$ of $\mathcal{C}^{+}\left(\bar{\Delta}_{n}^{\#}\right)$ for which $\left[X^{+1}\right]=$ $[X]^{+1}$ in $\mathrm{ObC}^{+}\left(\bar{\Delta}_{n}^{\#}\right)$, and for which

$$
X \vartheta_{n}=1_{[X]^{+1}}=1_{\left[X^{+1}\right]} \quad\left(\text { equality in } \underline{\mathcal{C}^{+}\left(\bar{\Delta}_{n}^{\#}\right)}\right) .
$$

A morphism of $\boldsymbol{n}$-triangles is a morphism $X \stackrel{u}{\longrightarrow} Y$ in $\mathcal{C}^{+}\left(\bar{\Delta}_{n}^{\#}\right)$ between $n$-triangles $X$ and $Y$ such that $[u]^{+1}=\left[u^{+1}\right]$.

The category of $n$-triangles and morphisms of $n$-triangles is denoted by $\mathcal{C}^{+, \vartheta=1}\left(\bar{\Delta}_{n}^{\#}\right)$.

(iii) An additive functor $\mathcal{C} \stackrel{F}{\longrightarrow} \mathcal{C}^{\prime}$ between Heller triangulated categories $(\mathcal{C}, T, \vartheta)$ and $\left(\mathcal{C}^{\prime}, T^{\prime}, \vartheta^{\prime}\right)$ is called strictly exact if the following conditions hold.

(1) $F \mathrm{~T}^{\prime}=\mathrm{T} F$.

(2) $F$ is subexact; cf. $§ 1.2 .1 .3$.

(3) We have

$$
\vartheta_{n} \star \underline{F^{+}\left(\bar{\Delta}_{n}^{\#}\right)}=\underline{F^{+}\left(\bar{\Delta}_{n}^{\#}\right)} \star \vartheta_{n}^{\prime}
$$

for all $n \geqslant 0$.

Remark 1.6. Some remarks concerning Definition 1.5 are in order. 
(i) Given $n \geqslant 0$, the isomorphism $\vartheta_{n}$ consists of isomorphisms

$$
[X]^{+1} \stackrel{X \vartheta_{n}}{\sim}\left[X^{+1}\right]
$$

in the stable category $\mathcal{C}^{+}\left(\bar{\Delta}_{n}^{\#}\right)$ of $n$-pretriangles, where $X$ runs over the set $\mathrm{Ob} \underline{\mathcal{C}^{+}\left(\bar{\Delta}_{n}^{\#}\right)}$ of $n$-pretriangles.

Condition $(*)$ asserts that the following diagram commutes in Add for all $n, m \geqslant 0$ and all periodic monotone maps $\bar{\Delta}_{n} \stackrel{p}{\longleftarrow} \bar{\Delta}_{m}$ in $\bar{\Delta}$.

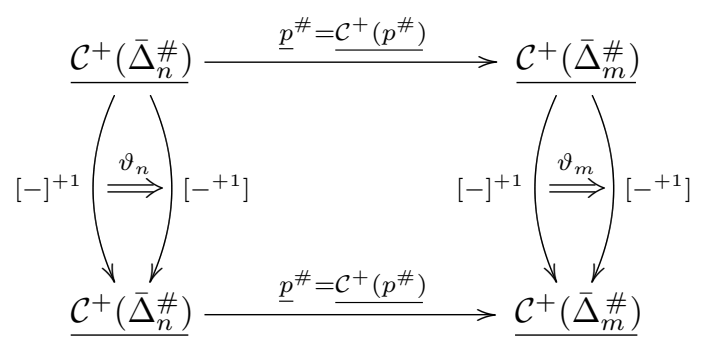

Condition (**) asserts that the following diagram commutes in Add for all $n \geqslant 0$.

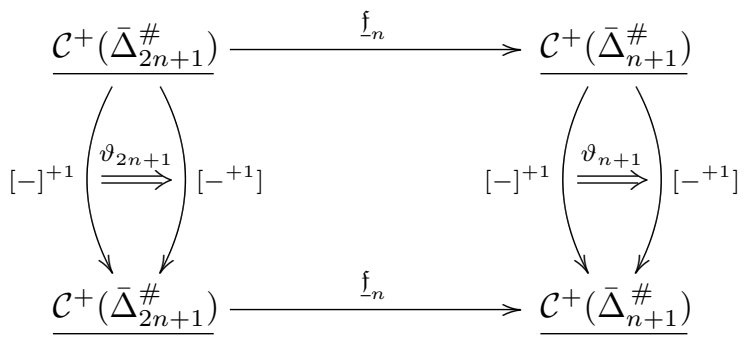

(ii) In the notation $\mathcal{C}^{+, \vartheta=1}\left(\bar{\Delta}_{n}^{\#}\right)$, the index " $\vartheta=1$ " is to be read as a symbol, not as an actual equation. The subcategory of $n$-triangles $\mathcal{C}^{+, \vartheta=1}\left(\bar{\Delta}_{n}^{\#}\right)$ in the category of $n$-pretriangles $\mathcal{C}^{+}\left(\bar{\Delta}_{n}^{\#}\right)$ is not full in general.

(iii) A functor $F$ as in Definition 1.5.(iii) is called strictly exact because of the equality in loc. cit. (1).

Condition $(* * *)$ asserts that the following diagram commutes in Add for all $n \geqslant 0$.

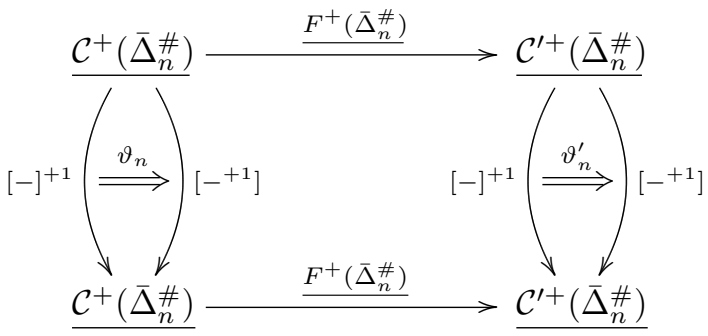


Homology, Homotopy and Applications, vol. 9(2), 2007

We summarise Definition 1.5 roughly.

(i) A Heller triangulation is an isomorphism $\vartheta$ from the outer shift to the inner shift, varying with $\Delta_{n}$, and compatible with folding.

(ii) An $n$-triangle is a periodic $n$-pretriangle at which $\vartheta$ is an identity.

(iii) A strictly exact functor respects the weakly abelian structure and is compatible with shift and $\vartheta$.

Note that if $\vartheta$ is a Heller triangulation on $(\mathcal{C}, T)$, then so is $-\vartheta$.

Definition 1.5 would make sense for periodic, but not necessarily linearly ordered posets, generalising $\bar{\Delta}_{n}$. But then it is unknown whether - and it seems to the author not very probable - the stable category of a Frobenius category is triangulated in this generalised sense. More specifically, it seems to be impossible to generalise Proposition 2.5 below accordingly, which is the technical core of our approach.

Question 1.7. Does there exist an additive functor $\mathcal{C} \stackrel{F}{\longrightarrow} \mathcal{C}^{\prime}$ between Heller triangulated categories that, in Definition 1.5.(iii), satisfies (1) and (2), but (3) only for $n \leqslant 2$ ? If $F$ is an identity, this amounts to asking for the existence of two Heller triangulations $\vartheta$ and $\vartheta^{\prime}$ on $(\mathcal{C}, T), \mathcal{C}$ weakly abelian, T automorphism of $\mathcal{C}$, such that $\vartheta_{n}=\vartheta_{n}^{\prime}$ only for $n \leqslant 2$.

\section{Some equivalences}

Suppose given $n \geqslant 0$. Suppose given a weakly abelian category $\mathcal{C}$, together with an automorphism $\mathrm{T}: \mathcal{C} \longrightarrow \mathcal{C}, X \longmapsto X^{+1}$. Concerning the Freyd category $\hat{\mathcal{C}}$ of $\mathcal{C}$, we refer to $§$ A.6.3.

We shall show in Proposition 2.6 that the functor $\mathcal{C}\left(\bar{\Delta}_{n}^{\#}\right) \longrightarrow \mathcal{C}\left(\dot{\Delta}_{n}\right)$, induced on the stable categories by restriction from $\bar{\Delta}_{n}^{\#}$ to $\dot{\Delta}_{n}:=[\overline{1, n}]$, is an equivalence.

\subsection{Some notation}

\subsubsection{SOME POSETS}

Let $\dot{\Delta}_{n}:=\Delta_{n} \backslash\{0\}=[1, n]$, considered as a linearly ordered set. We have an injection $\dot{\Delta}_{n} \longrightarrow \bar{\Delta}_{n}^{\#}, i \longmapsto i / 0$, and identify $\dot{\Delta}_{n}$ with its image in $\bar{\Delta}_{n}^{\#}$.

We define two subposets of $\bar{\Delta}_{n}^{\#}$ by

$$
\begin{aligned}
& \bar{\Delta}_{n}^{\Delta}:=\left\{\beta / \alpha \in \bar{\Delta}_{n}^{\#}: 0 \leqslant \alpha\right\} \\
& \bar{\Delta}_{n}^{\nabla}:=\left\{\beta / \alpha \in \bar{\Delta}_{n}^{\#}: \alpha \leqslant 0\right\} .
\end{aligned}
$$

Then $\dot{\Delta}_{n}=\left(\bar{\Delta}_{n}^{\Delta} \cap \bar{\Delta}_{n}^{\nabla}\right) \backslash\left\{0 / 0,0^{+1} / 0\right\}$. 


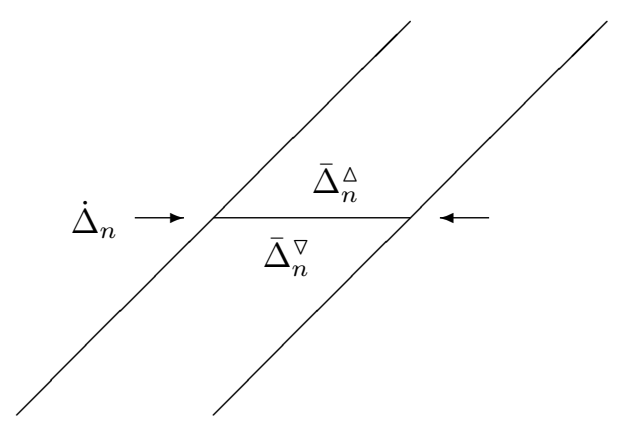

\subsubsection{FIXING PARAMETERISATIONS $\kappa^{\Delta}, \kappa^{\nabla}$}

There exists a bijective morphism $\bar{\Delta}_{n}^{\Delta} \longrightarrow \mathbf{Z}_{\geqslant 0}$ of posets ("refining the partial to a linear order"). We fix such a morphism and denote by $\mathbf{Z}_{\geqslant 0} \stackrel{\kappa^{\Delta}}{\longrightarrow} \bar{\Delta}_{n}^{\Delta}$ its inverse (as a map of sets; in general, $\kappa^{\Delta}$ is not monotone). So whenever $\kappa^{\Delta}(l) \leqslant \kappa^{\Delta}\left(l^{\prime}\right)$, then $l \leqslant l^{\prime}$. In particular, $\kappa^{\Delta}(0)=0 / 0$.

There exists a bijective morphism $\bar{\Delta}_{n}^{\nabla} \longrightarrow \mathbf{Z}_{\leqslant 0}$ of posets. We fix such a morphism and denote by $\mathbf{Z}_{\geqslant 0} \stackrel{\kappa^{\nabla}}{\longrightarrow} \bar{\Delta}_{n}^{\nabla}$ its inverse (as a map of sets). So whenever $\kappa^{\nabla}(l) \leqslant$ $\kappa^{\nabla}\left(l^{\prime}\right)$, then $l \leqslant l^{\prime}$. In particular, $\kappa^{\nabla}(0)=0^{+1} / 0$.

\subsubsection{The CAtegories $\hat{\mathcal{C}}^{+, *}\left(\bar{\Delta}_{n}^{\Delta}\right), \mathcal{C}^{+}\left(\bar{\Delta}_{n}^{\Delta}\right)$, EtC.}

Let $\mathcal{A}$ be an abelian category, and let $\mathcal{B} \subseteq \mathcal{A}$ be a full subcategory. Let $E \subseteq \bar{\Delta}_{n}^{\#}$ be a full subposet.

For example, for $E$ we may take the subposets $\bar{\Delta}_{n}^{\Delta}, \bar{\Delta}_{n}^{\nabla}$ or $\bar{\Delta}_{n}^{\Delta} \cap \bar{\Delta}_{n}^{\nabla,+1}$ of $\bar{\Delta}_{n}^{\#}$.

Moreover, for example, we may take $\mathcal{A}=\hat{\mathcal{C}}$, and for $\mathcal{B}$ either $\hat{\mathcal{C}}$ or $\mathcal{C}$.

Let $\mathcal{B}^{+, *}(E)$ be the full subcategory of $\mathcal{B}(E)$ defined by

$$
\operatorname{ObB}^{+, *}(E):=\left\{\begin{array}{ll} 
& \text { For all } \delta^{-1} \leqslant \alpha \leqslant \beta \leqslant \gamma \leqslant \delta \leqslant \alpha^{+1} \\
& \text { in } \bar{\Delta}_{n} \text { such that } \gamma / \alpha, \gamma / \beta, \delta / \alpha \\
& \text { and } \delta / \beta \text { are in } E, \text { the quadrangle } \\
& X_{\gamma / \beta} \longrightarrow X_{\delta / \beta} \\
& x \uparrow_{x}+\uparrow_{x} \\
& X_{\gamma / \alpha} \stackrel{x}{\longrightarrow} X_{\delta / \alpha} \\
& \text { is a weak square (as indicated by }+ \text { ). }
\end{array}\right\}
$$

The symbol $*$ should remind us of the fact that we still allow $X_{\alpha / \alpha}$ resp. $X_{\alpha^{+1} / \alpha}$ to be arbitrary for $\alpha \in \bar{\Delta}_{n}$ such that $\alpha / \alpha \in E$ resp. $\alpha^{+1} / \alpha \in E$.

In turn, let $\mathcal{B}^{+}(E)$ be the full subcategory of $\mathcal{B}^{+, *}(E)$ defined by

$$
\mathrm{ObB}^{+}(E):=\left\{\begin{array}{ll} 
& X_{\alpha / \alpha}=0 \text { for } \alpha \in \bar{\Delta}_{n} \text { such that } \\
X \in \mathrm{ObB}^{+, *}(E): & \alpha / \alpha \in E, \text { and } X_{\alpha+1} / \alpha=0 \text { for } \\
& \alpha \in \bar{\Delta}_{n} \text { such that } \alpha^{+1} / \alpha \in E .
\end{array}\right\}
$$




\subsubsection{REINDEXING}

Given a subposet $E \subseteq \bar{\Delta}_{n}^{\#}$, we have a reindexing equivalence

$$
\begin{aligned}
\mathcal{C}(E) & \stackrel{\sim}{\longrightarrow}\left(E^{+1}\right) \\
X & \longmapsto X^{(-1)} \\
X^{(+1)} & \hookrightarrow X
\end{aligned}
$$

defined by

$$
\left(X^{(-1)}\right)_{\beta / \alpha}:=X_{(\beta / \alpha)^{-1}}=X_{\alpha / \beta^{-1}},
$$

where $\beta / \alpha \in E^{+1}$; and inversely by

$$
\left(X^{(+1)}\right)_{\beta / \alpha}:=X_{(\beta / \alpha)+1}=X_{\alpha+1 / \beta},
$$

where $\beta / \alpha \in E$. This equivalence restricts to an equivalence between $\mathcal{C}^{+}(E)$ and $\mathcal{C}^{+}\left(E^{+1}\right)$.

For instance, if $E=\bar{\Delta}_{n}^{\#}$, then $X^{(+1)}=[X]^{+1}$. The outer shift and reindexing will play different roles, and so we distinguish in notation.

2.2. Density of the restriction functor from $\bar{\Delta}_{n}^{\#}$ to $\dot{\Delta}_{n}$

2.2.1 UPWARDS AND DOWNWARDS SPREAD

Let the upwards spread $S^{\Delta}$ be defined by

$$
\begin{aligned}
\hat{\mathcal{C}}\left(\dot{\Delta}_{n}\right) & \stackrel{S^{\Delta}}{\longrightarrow} \hat{\mathcal{C}}^{+}\left(\bar{\Delta}_{n}^{\Delta}\right) \\
X & \longmapsto X S^{\Delta},
\end{aligned}
$$

where $X S^{\triangle}$ is given by

$$
\begin{array}{ll}
\left(X S^{\Delta}\right)_{0 / 0}:=0 & \text { for } \beta \in \dot{\Delta}_{n} \\
\left(X S^{\Delta}\right)_{\beta / 0}:=X_{\beta} & \text { for } \alpha, \beta \in \dot{\Delta}_{n} \text { with } \alpha \leqslant \beta \\
\left(X S^{\Delta}\right)_{\beta / \alpha}:=\operatorname{Cokern}\left(X_{\alpha} \longrightarrow X_{\beta}\right) & \text { for } \alpha, \beta \in \bar{\Delta}_{n} \text { with } 0^{+1} \leqslant \beta \leqslant \alpha^{+1} \leqslant \beta^{+1},
\end{array}
$$

the diagram being completed with the induced morphisms between the cokernels and zero morphisms elsewhere.

This construction is functorial in $X$. The functor $S^{\Delta}$ is left adjoint to the restriction functor from $\hat{\mathcal{C}}^{+}\left(\bar{\Delta}_{n}^{\Delta}\right)$ to $\hat{\mathcal{C}}\left(\dot{\Delta}_{n}\right)$, with unit being the identity, i.e. $X=\left.X S^{\Delta}\right|_{\dot{\Delta}_{n}}$.

Dually, let the downwards spread $S^{\nabla}$ be

$$
\begin{aligned}
\hat{\mathcal{C}}\left(\dot{\Delta}_{n}\right) & \stackrel{S^{\nabla}}{\longrightarrow} \hat{\mathcal{C}}^{+}\left(\bar{\Delta}_{n}^{\nabla}\right) \\
X & \longmapsto X S^{\nabla},
\end{aligned}
$$

where $X S^{\nabla}$ is given by

$$
\begin{array}{rlrl}
\left(X S^{\nabla}\right)_{0^{+1} / 0} & :=0 & & \\
\left(X S^{\nabla}\right)_{\alpha / 0} & :=X_{\alpha} & & \text { for } \alpha \in \dot{\Delta}_{n} \\
\left(X S^{\nabla}\right)_{\alpha / \beta^{-1}} & :=\operatorname{Kern}\left(X_{\alpha} \stackrel{x}{\longrightarrow} X_{\beta}\right) & \text { for } \alpha, \beta \in \dot{\Delta}_{n} \text { with } \alpha \leqslant \beta \\
\left(X S^{\nabla}\right)_{\alpha / \beta^{-1}} & :=0 & & \text { for } \alpha, \beta \in \bar{\Delta}_{n} \text { with } \alpha^{-1} \leqslant \beta^{-1} \leqslant \alpha \leqslant 0,
\end{array}
$$


the diagram being completed with the induced morphisms between the kernels and zero morphisms elsewhere.

This construction is functorial in $X$. The functor $S^{\nabla}$ is right adjoint to the restriction functor from $\hat{\mathcal{C}}^{+}\left(\bar{\Delta}_{n}^{\nabla}\right)$ to $\hat{\mathcal{C}}\left(\dot{\Delta}_{n}\right)$, with counit being the identity, i.e. $\left.X S^{\nabla}\right|_{\dot{\Delta}_{n}}=X$.

\subsubsection{Resolutions}

\subsubsection{A STABILITY UNDER POINTWISE PUSHOUTS AND PULLBACKS}

Let $E \subseteq \bar{\Delta}_{n}^{\#}$ be a full subposet. Moreover, assume that $E$ is a convex subposet, i.e. that whenever given $\xi, \zeta \in E$ and $\eta \in \bar{\Delta}_{n}^{\#}$ such that $\xi \leqslant \eta \leqslant \zeta$, then $\eta \in E$.

An element $\delta / \beta \in E$ is on the left boundary of $\boldsymbol{E}$ if we may conclude from $\gamma / \beta \in E$ and $\gamma \leqslant \delta$ that $\gamma=\delta$. It is on the lower boundary of $\boldsymbol{E}$ if we may conclude from $\delta / \alpha \in E$ and $\alpha \leqslant \beta$ that $\alpha=\beta$.

An element $\gamma / \alpha \in E$ is on the right boundary of $\boldsymbol{E}$ if we may conclude from $\delta / \alpha \in E$ and $\gamma \leqslant \delta$ that $\gamma=\delta$. It is on the upper boundary of $\boldsymbol{E}$ if we may conclude from $\gamma / \beta \in E$ and $\alpha \leqslant \beta$ that $\alpha=\beta$.

Let $\mathcal{A}$ be an abelian category. Concerning pointwise pullbacks and pointwise pushouts, we refer to $\S$ A.7.

Lemma 2.1. Suppose given $\varepsilon \in E$ and an object $X$ of $\mathcal{A}^{+, *}(E)$.

(1) Given a monomorphism $X_{\varepsilon} \stackrel{x^{\prime}}{\longrightarrow} X^{\prime}$ in $\mathcal{A}$, the pointwise pushout $X \uparrow^{x^{\prime}}$ of $X$ along $x^{\prime}$ is an object of $\mathcal{A}^{+, *}(E)$ again.

(2) Given an epimorphism $X_{\varepsilon} \stackrel{x^{\prime}}{\longleftarrow} X^{\prime}$ in $\mathcal{A}$, the pointwise pullback $X \Upsilon_{x^{\prime}}$ of $X$ along $x^{\prime}$ is an object of $\mathrm{Ob} \mathcal{A}^{+, *}(E)$ again.

(3) Suppose that $\varepsilon$ is on the left boundary or on the lower boundary of E. Given a morphism $X_{\varepsilon} \stackrel{x^{\prime}}{\longrightarrow} X^{\prime}$ in $\mathcal{A}$, the pointwise pushout $X \uparrow^{x^{\prime}}$ of $X$ along $x^{\prime}$ is an object of $\mathcal{A}^{+, *}(E)$ again.

(4) Suppose that $\varepsilon$ is on the right boundary or on the upper boundary of E. Given a morphism $X_{\varepsilon} \stackrel{x^{\prime}}{\longleftarrow} X^{\prime}$ in $\mathcal{A}$, the pointwise pullback $X \uparrow_{x^{\prime}}$ of $X$ along $x^{\prime}$ is an object of $\mathrm{Ob} \mathcal{A}^{+, *}(E)$ again.

Proof. Ad (1). First we remark that by Lemma A.15, the quadrangle $\left(X_{\beta / \alpha}, X_{\delta / \gamma}\right.$, $\left.\left(X \uparrow^{x^{\prime}}\right)_{\beta / \alpha},\left(X \uparrow^{x^{\prime}}\right)_{\delta / \gamma}\right)$ is a pushout for $\varepsilon \leqslant \beta / \alpha \leqslant \delta / \gamma$ in $E$.

We have to show that the quadrangle of $X \uparrow^{x^{\prime}}$ on $(\gamma / \alpha, \delta / \alpha, \gamma / \beta, \delta / \beta)$, where $\delta^{-1} \leqslant \alpha \leqslant \beta \leqslant \gamma \leqslant \delta \leqslant \alpha^{+1}$ in $\bar{\Delta}_{n}$, is a weak square, provided its corners have indices in $E$. Using Lemmata A.14, A.16 and convexity of $E$, we are reduced to the case $\varepsilon \leqslant \gamma / \alpha$. In this case, the assertion follows by Lemma A.15.

Ad (3). Here we need only Lemma A.14 and convexity of $E$ to reduce to the case $\varepsilon \leqslant \gamma / \alpha$; the rest of the argument is as in (1). Hence the morphism $x^{\prime}$ may be arbitrary. 


\subsubsection{UPWARDS AND DOWNWARDS RESOLUTION}

Remark 2.2.

(1) Given a direct system $X_{0} \longrightarrow X_{1} \longrightarrow X_{2} \longrightarrow \cdots$ in $\hat{\mathcal{C}}^{+, *}\left(\bar{\Delta}_{n}^{\Delta}\right)$ such that its restriction to any finite full subposet $E \subseteq \bar{\Delta}_{n}^{\Delta}$ eventually becomes constant, then the direct limit $\lim _{i} X_{i}$ exists in $\hat{\mathcal{C}}^{+, *}\left(\bar{\Delta}_{n}^{\Delta}\right)$.

(2) Given an inverse system $X_{0} \longleftarrow X_{1} \longleftarrow X_{2} \longleftarrow \cdots$ in $\hat{\mathcal{C}}^{+, *}\left(\bar{\Delta}_{n}^{\nabla}\right)$ such that its restriction to any finite subposet $E \subseteq \bar{\Delta}_{n}^{\nabla}$ eventually becomes constant, then the inverse limit $\lim _{i} X_{i}$ exists in $\hat{\mathcal{C}}^{+, *}\left(\bar{\Delta}_{n}^{\nabla}\right)$.

For $k \geqslant 0$, we let

$$
\begin{aligned}
\operatorname{Ob} \hat{\mathcal{C}}^{+, *}\left(\bar{\Delta}_{n}^{\Delta}\right) \stackrel{R_{k}^{\Delta}}{\longrightarrow} \operatorname{Ob} \hat{\mathcal{C}}^{+, *}\left(\bar{\Delta}_{n}^{\Delta}\right) \\
X \longmapsto X \uparrow^{\prime}(k)
\end{aligned}
$$

where

$$
x^{\prime}(k):=\left\{\begin{array}{cc}
\left(X_{\kappa^{\Delta}(k)} \longrightarrow 0\right) & \text { if } \kappa^{\Delta}(k) \in\left\{\alpha / \alpha, \alpha^{+1} / \alpha\right\} \\
& \text { for some } \alpha \in \bar{\Delta}_{n} \text { with } 0 \leqslant \alpha \\
\left(X_{\kappa^{\Delta}(k)} \longrightarrow X_{\kappa^{\Delta}(k)} I\right) & \text { if } \kappa^{\Delta}(k)=\beta / \alpha \text { for some } \alpha, \beta \in \bar{\Delta}_{n} \\
\text { with } 0 \leqslant \alpha<\beta<\alpha^{+1} .
\end{array}\right.
$$

Define the upwards resolution map by

$$
\begin{aligned}
& \mathrm{Ob} \hat{\mathcal{C}}^{+, *}\left(\bar{\Delta}_{n}^{\Delta}\right) \stackrel{R^{\Delta}}{\longrightarrow} \mathrm{ObC}^{+}\left(\bar{\Delta}_{n}^{\Delta}\right) \\
& X \longmapsto X R^{\Delta}:=\underset{m}{\lim _{m}} X R_{0}^{\Delta} \cdots R_{m}^{\Delta},
\end{aligned}
$$

the direct system being given by the transition morphisms

$$
X R_{0}^{\Delta} \cdots R_{m}^{\Delta} \stackrel{i}{\longrightarrow}\left(X R_{0}^{\Delta} \cdots R_{m}^{\Delta}\right) R_{m+1}^{\Delta} .
$$

We have $X R^{\Delta}=X$ for $X \in \mathrm{ObC}^{+}\left(\bar{\Delta}_{n}^{\Delta}\right)$.

Note that we apparently cannot turn the upwards resolution into a functor unless we are in a particular case in which the map I on objects can be turned into a functor.

Dually, for $k \geqslant 0$, we let

$$
\begin{aligned}
\mathrm{Ob} \hat{\mathcal{C}}^{+, *}\left(\bar{\Delta}_{n}^{\nabla}\right) & \stackrel{R_{k}^{\nabla}}{\longmapsto} \mathrm{Ob} \hat{\mathcal{C}}^{+, *}\left(\bar{\Delta}_{n}^{\nabla}\right) \\
X & \longmapsto X \uparrow_{x^{\prime \prime}(k)}
\end{aligned}
$$

where

$$
x^{\prime \prime}(k):= \begin{cases}\left(X_{\left.\kappa^{\nabla}(k) \longleftarrow 0\right)}{ }\right) & \text { if } \kappa^{\nabla}(k) \in\left\{\alpha / \alpha, \alpha^{+1} / \alpha\right\} \\ & \text { for some } \alpha \in \bar{\Delta}_{n} \text { with } \alpha \leqslant 0 . \\ \left(X_{\kappa^{\nabla}(k)} \stackrel{\pi}{\longleftarrow} X_{\kappa^{\nabla}(k)} \mathrm{P}\right) & \text { if } \kappa^{\nabla}(k)=\beta / \alpha \text { for some } \alpha, \beta \in \bar{\Delta}_{n} \\ & \text { with } \alpha^{-1}<\beta^{-1}<\alpha \leqslant 0 .\end{cases}
$$

Define the downwards resolution map by

$$
\begin{aligned}
\mathrm{Ob} \hat{\mathcal{C}}^{+, *}\left(\bar{\Delta}_{n}^{\nabla}\right) & \stackrel{R^{\nabla}}{\longmapsto} \mathrm{ObC}^{+}\left(\bar{\Delta}_{n}^{\nabla}\right) \\
X & \longmapsto X R^{\nabla}:={\underset{m}{m}}_{\lim _{0}} X R_{0}^{\nabla} \cdots R_{m}^{\nabla},
\end{aligned}
$$


Homology, Homotopy and Applications, vol. 9(2), 2007

the inverse system being given by the transition morphisms

$$
X R_{0}^{\nabla} \cdots R_{m}^{\nabla} \stackrel{p}{\longleftarrow}\left(X R_{0}^{\nabla} \cdots R_{m}^{\nabla}\right) R_{m+1}^{\nabla} .
$$

We have $X R^{\nabla}=X$ for $X \in \mathrm{ObC}^{+}\left(\bar{\Delta}_{n}^{\nabla}\right)$.

\section{Lemma 2.3.}

(1) Given a morphism $Y \stackrel{g}{\longrightarrow} X$ in $\hat{\mathcal{C}}^{+}\left(\bar{\Delta}_{n}^{\Delta}\right)$ with $X \in \mathrm{Ob}^{+}\left(\bar{\Delta}_{n}^{\Delta}\right)$, there exists a factorisation

$$
(Y \stackrel{g}{\longrightarrow} X)=\left(Y \longrightarrow Y R^{\Delta} \longrightarrow X\right)
$$

(2) Given a morphism $Y \stackrel{g}{\longleftarrow} X$ in $\hat{\mathcal{C}}^{+}\left(\bar{\Delta}_{n}^{\nabla}\right)$ with $X \in \mathrm{ObC}^{+}\left(\bar{\Delta}_{n}^{\nabla}\right)$, there exists a factorisation

$$
\left(Y \longleftarrow \frac{g}{\longleftarrow} X\right)=\left(Y \longleftarrow Y R^{\nabla} \longleftarrow X\right) .
$$

Proof. Ad (1). Since the entries of $X$ are injective in $\hat{\mathcal{C}}$ and since $X_{\alpha / \alpha}=0$ for $\alpha \geqslant 0$, we obtain, using the universal property of the pointwise pushout, a factorisation

$$
(Y \stackrel{g}{\longrightarrow} X)=\left(Y \longrightarrow Y R_{0}^{\Delta} \cdots R_{m}^{\Delta} \longrightarrow X\right)
$$

for every $m \geqslant 0$, compatible with the transition morphisms, resulting in a factorisation over $Y R^{\Delta}=\lim _{m} Y R_{0}^{\Delta} \cdots R_{m}^{\Delta}$.

\subsubsection{BOTH-SIDED RESOLUTIONS}

Let the resolution map

$$
\begin{aligned}
\mathrm{ObC}\left(\dot{\Delta}_{n}\right) & \stackrel{R}{\longrightarrow} \mathrm{ObC}^{+}\left(\bar{\Delta}_{n}^{\#}\right) \\
X & \longmapsto X R
\end{aligned}
$$

be defined by gluing an upper and a lower part along $\dot{\Delta}_{n}$ as follows.

$$
\begin{aligned}
\left.X R\right|_{\bar{\Delta}_{n}^{\Delta}} & :=X S^{\Delta} R^{\Delta} \\
\left.X R\right|_{\bar{\Delta}_{n}^{\nabla}} & :=X S^{\nabla} R^{\nabla}
\end{aligned}
$$

This is well defined, since $\left.X S^{\Delta} R^{\Delta}\right|_{\dot{\Delta}_{n}}=X=\left.X S^{\nabla} R^{\nabla}\right|_{\dot{\Delta}_{n}}$. In particular, we obtain

$$
\left.X R\right|_{\dot{\Delta}_{n}}=X
$$

We summarise.

Proposition 2.4. The restriction functor

$$
\begin{aligned}
\mathcal{C}^{+}\left(\bar{\Delta}_{n}^{\#}\right) & \stackrel{\left.(-)\right|_{\dot{\Delta}_{n}}}{\longrightarrow} \mathcal{C}\left(\dot{\Delta}_{n}\right) \\
Y & \left.\longmapsto Y\right|_{\dot{\Delta}_{n}}
\end{aligned}
$$

is strictly dense, i.e. it is surjective on objects. 
Homology, Homotopy and Applications, vol. 9(2), 2007

\subsection{Fullness of the restriction functor from $\bar{\Delta}_{n}^{\#}$ to $\dot{\Delta}_{n}$}

Proposition 2.5. The restriction functors

$$
\begin{aligned}
\mathcal{C}^{+}\left(\bar{\Delta}_{n}^{\#}\right) \stackrel{\left.(-)\right|_{\dot{\Delta}_{n}}}{\longrightarrow} \mathcal{C}\left(\dot{\Delta}_{n}\right) \\
\left.Y \longmapsto Y\right|_{\dot{\Delta}_{n}}
\end{aligned}
$$

and

$$
\begin{aligned}
\mathcal{C}^{+}\left(\bar{\Delta}_{n}^{\Delta}\right) & \stackrel{\left.(-)\right|_{\dot{\Delta}_{n}}}{\longrightarrow} \mathcal{C}\left(\dot{\Delta}_{n}\right) \\
Y & \left.\longmapsto Y\right|_{\dot{\Delta}_{n}}
\end{aligned}
$$

are full.

Proof. By duality and gluing along $\dot{\Delta}_{n}$, it suffices to consider the restriction from $\bar{\Delta}_{n}^{\Delta}$ to $\dot{\Delta}_{n}$. So suppose given $X, Y \in \mathrm{ObC}^{+}\left(\bar{\Delta}_{n}^{\Delta}\right)$ and a morphism $\left.\left.X\right|_{\dot{\Delta}_{n}} \stackrel{f}{\longrightarrow} Y\right|_{\dot{\Delta}_{n}}$. We have to find a morphism $X \stackrel{f^{\Delta}}{\longrightarrow} Y$ such that $\left.f^{\Delta}\right|_{\dot{\Delta}_{n}}=f$.

We construct $f_{\kappa^{\Delta}(\ell)}^{\Delta}$ for $\ell \geqslant 0$ by induction on $\ell$.

At $\kappa^{\Delta}(0)$, we let $f_{\kappa^{\Delta}(0)}^{\Delta}:=1_{0}$. Suppose given $\ell \geqslant 1$. If $\kappa^{\Delta}(\ell) \in \dot{\Delta}_{n}$, we let $f_{\kappa^{\Delta}(\ell)}^{\Delta}:=$ $f_{\kappa^{\Delta}(\ell)}$. If $\kappa^{\Delta}(\ell) \in\left\{\alpha / \alpha, \alpha^{+1} / \alpha\right\}$ for some $\alpha \geqslant 0$, we let $f_{\kappa^{\Delta}(\ell)}^{\Delta}:=1_{0}$. If $\kappa^{\Delta}(\ell)=: \beta / \alpha$ with $0<\alpha<\beta<\alpha^{+1}$, then we let $\alpha^{\prime}:=\alpha-1$ be the predecessor of $\alpha$ in $\bar{\Delta}_{n}$, and we let $\beta^{\prime}:=\beta-1$ be the predecessor of $\beta$ in $\bar{\Delta}_{n}$, using that $\bar{\Delta}_{n}$ is linearly ordered. We may complete the diagram

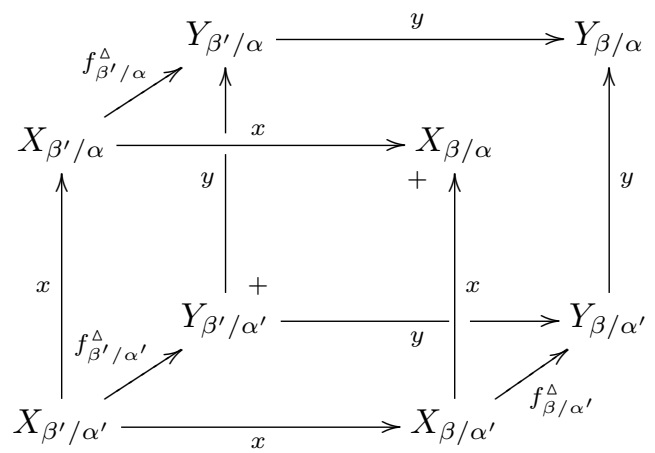

to a commutative cuboid, inserting a morphism $X_{\beta / \alpha} \stackrel{f_{\beta / \alpha}^{\Delta}}{\longrightarrow} Y_{\beta / \alpha}$.

Since we need the restriction functor $\mathcal{C}^{+}\left(\bar{\Delta}_{n}^{\#}\right) \stackrel{\left.(-)\right|_{\Delta_{n}}}{\longrightarrow} \mathcal{C}\left(\dot{\Delta}_{n}\right)$ to be full, we are not able to generalise from linearly ordered periodic posets to arbitrary periodic posets.

\subsection{The equivalence between $\mathcal{C}^{+}\left(\bar{\Delta}_{n}^{\#}\right)$ and $\mathcal{C}\left(\dot{\Delta}_{n}\right)$}

Let $\mathcal{C}^{\text {split }}\left(\dot{\Delta}_{n}\right)$ be the full subcategory of $\mathcal{C}\left(\dot{\Delta}_{n}\right)$ defined by

$$
\operatorname{Ob} \mathcal{C}^{\text {split }}\left(\dot{\Delta}_{n}\right):=\left\{X \in \operatorname{ObC}\left(\dot{\Delta}_{n}\right): \begin{array}{l}
X_{\alpha} \longrightarrow X_{\beta} \text { is split } \\
\text { for all } \alpha, \beta \in \dot{\Delta}_{n} \text { with } \alpha \leqslant \beta
\end{array}\right\} .
$$


We denote the quotient category by

$$
\underline{\mathcal{C}\left(\dot{\Delta}_{n}\right)}:=\mathcal{C}\left(\dot{\Delta}_{n}\right) / \mathcal{C}^{\text {split }}\left(\dot{\Delta}_{n}\right)
$$

Proposition 2.6. The functor

$$
\begin{aligned}
& \underline{\mathcal{C}^{+}\left(\bar{\Delta}_{n}^{\#}\right)} \stackrel{\left.(-)\right|_{\dot{\Delta}_{n}}}{\longrightarrow} \underline{\mathcal{C}\left(\dot{\Delta}_{n}\right)} \\
& \left.X \longmapsto X\right|_{\dot{\Delta}_{n}},
\end{aligned}
$$

induced by restriction from $\bar{\Delta}_{n}^{\#}$ to $\dot{\Delta}_{n}$, is an equivalence.

Proof. By Propositions 2.4 and 2.5, we may invoke Lemma A.1. Moreover, Lemma 1.4 gives the inverse image of $\operatorname{Ob} \mathcal{C}^{\text {split }}\left(\dot{\Delta}_{n}\right)$ under $\mathcal{C}^{+}\left(\bar{\Delta}_{n}^{\#}\right) \stackrel{\left.(-)\right|_{\dot{\Delta}_{n}}}{\longrightarrow} \mathcal{C}\left(\dot{\Delta}_{n}\right)$ as $\operatorname{Ob} \mathcal{C}^{+, \operatorname{split}}\left(\bar{\Delta}_{n}^{\#}\right)$.

Consider a morphism $X \stackrel{f}{\longrightarrow} X^{\prime}$ in $\mathcal{C}^{+}\left(\bar{\Delta}_{n}^{\#}\right)$ such that $\left.\left(X \stackrel{f}{\longrightarrow} X^{\prime}\right)\right|_{\dot{\Delta}_{n}}$ is zero in $\mathcal{C}\left(\dot{\Delta}_{n}\right)$. We have to prove that it factors over an object of $\mathcal{C}^{+, \text {split }}\left(\bar{\Delta}_{n}^{\#}\right)$.

Let $Y^{\Delta}$ be the cokernel in $\hat{\mathcal{C}}\left(\bar{\Delta}_{n}^{\Delta}\right)$ of the counit $\left.\left.X\right|_{\dot{\Delta}_{n}} S^{\Delta} \longrightarrow X\right|_{\bar{\Delta}_{n}^{\Delta}}$ at $\left.X\right|_{\bar{\Delta}_{n}^{\Delta}}$. Note that $\left.Y^{\Delta}\right|_{\dot{\Delta}_{n}}=0$. By Lemma A.21, we have $Y^{\Delta} \in \mathrm{Ob} \hat{\mathcal{C}}^{+}\left(\bar{\Delta}_{n}^{\Delta}\right)$. Consider the following diagram in $\hat{\mathcal{C}}^{+}\left(\bar{\Delta}_{n}^{\Delta}\right)$.

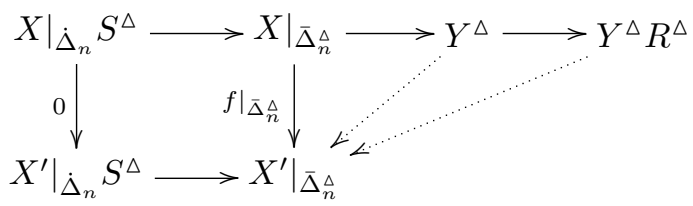

The indicated factorisation

$$
\left(\left.\left.X\right|_{\bar{\Delta}_{n}^{\Delta}} \stackrel{\left.f\right|_{\bar{\Delta}_{n}^{\Delta}}}{\longrightarrow} X^{\prime}\right|_{\bar{\Delta}_{n}^{\Delta}}\right)=\left(\left.\left.X\right|_{\bar{\Delta}_{n}^{\Delta}} \longrightarrow Y^{\Delta} \longrightarrow X^{\prime}\right|_{\bar{\Delta}_{n}^{\Delta}}\right)
$$

ensues from the universal property of the cokernel $Y^{\Delta}$. By Lemma 2.3.(1), we can factorise further to obtain

$$
\left(\left.\left.X\right|_{\bar{\Delta}_{n}^{\Delta}} \stackrel{\left.f\right|_{\bar{\Delta}_{n}^{\Delta}}}{\longrightarrow} X^{\prime}\right|_{\bar{\Delta}_{n}^{\Delta}}\right)=\left(\left.\left.X\right|_{\bar{\Delta}_{n}^{\Delta}} \longrightarrow Y^{\Delta} R^{\Delta} \longrightarrow X^{\prime}\right|_{\bar{\Delta}_{n}^{\Delta}}\right) .
$$

Dually, we obtain a factorisation

$$
\left(\left.\left.X\right|_{\bar{\Delta}_{n}^{\nabla}} \stackrel{\left.f\right|_{\bar{\Delta}_{n}^{\nabla}}}{\longrightarrow} X^{\prime}\right|_{\bar{\Delta}_{n}^{\nabla}}\right)=\left(\left.\left.X\right|_{\bar{\Delta}_{n}^{\nabla}} \longrightarrow Y^{\nabla} R^{\nabla} \longrightarrow X^{\prime}\right|_{\bar{\Delta}_{n}^{\nabla}}\right)
$$

for some $Y^{\nabla} \in \mathrm{Ob} \hat{\mathcal{C}}^{+}\left(\bar{\Delta}_{n}^{\nabla}\right)$ such that $\left.Y^{\nabla}\right|_{\dot{\Delta}_{n}}=0$.

Since $\left.Y^{\Delta} R^{\Delta}\right|_{\dot{\Delta}_{n}}=0=\left.Y^{\nabla} R^{\nabla}\right|_{\dot{\Delta}_{n}}$, there is a unique $N \in \mathrm{ObC}^{+}\left(\bar{\Delta}_{n}^{\#}\right)$ such that $\left.N\right|_{\bar{\Delta}_{n}^{\Delta}}=Y^{\Delta} R^{\Delta}$ and $\left.N\right|_{\bar{\Delta}_{n}^{\nabla}}=Y^{\nabla} R^{\nabla}$. By Lemma 1.4 , we have $N \in \operatorname{Ob} \mathcal{C}^{+, \text {split }}\left(\bar{\Delta}_{n}^{\#}\right)$.

Moreover, since both factorisations $\left(*^{\Delta}\right)$ and $\left(*^{\nabla}\right)$ restrict to the factorisation

$$
\left(\left.\left.X\right|_{\dot{\Delta}_{n}} \stackrel{0}{\longrightarrow} X^{\prime}\right|_{\dot{\Delta}_{n}}\right)=\left(\left.\left.X\right|_{\dot{\Delta}_{n}} \longrightarrow 0 \longrightarrow X^{\prime}\right|_{\dot{\Delta}_{n}}\right)
$$

in $\mathcal{C}\left(\dot{\Delta}_{n}\right)$, we may glue to a factorisation

$$
\left(X \stackrel{f}{\longrightarrow} X^{\prime}\right)=\left(X \longrightarrow N \longrightarrow X^{\prime}\right)
$$

that restricts to $\left(*^{\Delta}\right)$ in $\mathcal{C}^{+}\left(\bar{\Delta}_{n}^{\Delta}\right)$ and to $\left(*^{\nabla}\right)$ in $\mathcal{C}^{+}\left(\bar{\Delta}_{n}^{\nabla}\right)$. 
Homology, Homotopy and Applications, vol. 9(2), 2007

\subsection{Auxiliary equivalences}

We shall extend the equivalence $\mathcal{C}^{+}\left(\bar{\Delta}_{n}^{\#}\right) \stackrel{\sim}{\longrightarrow} \underline{\mathcal{C}\left(\dot{\Delta}_{n}\right)}$ to a diagram of equivalences

$$
\underline{\mathcal{C}^{+}\left(\bar{\Delta}_{n}^{\#}\right) \stackrel{\sim}{\longrightarrow} \underline{\mathcal{C}^{+}\left(\Delta_{n}^{\Delta \nabla}\right)} \stackrel{\sim}{\sim} \underline{\mathcal{C}\left(\dot{\Delta}_{n}\right)} \stackrel{\sim}{\sim} \hat{\mathcal{C}}\left(\dot{\Delta}_{n-1}\right)} .
$$

\subsubsection{FACTORISATION INTO TWO EQUIVALENCES}

Abbreviate

$$
\bar{\Delta}_{n}^{\Delta,+1}:=\left(\bar{\Delta}_{n}^{\Delta}\right)^{+1} \subseteq \bar{\Delta}_{n}^{\#}, \bar{\Delta}_{n}^{\nabla,+1}:=\left(\bar{\Delta}_{n}^{\nabla}\right)^{+1} \subseteq \bar{\Delta}_{n}^{\#} \text { and } \dot{\Delta}_{n}^{+1}:=\left(\dot{\Delta}_{n}\right)^{+1} \subseteq \bar{\Delta}_{n}^{\#} .
$$

Abbreviate $\bar{\Delta}_{n}^{\Delta \nabla}:=\bar{\Delta}_{n}^{\Delta} \cap \bar{\Delta}_{n}^{\nabla,+1}=\left\{\beta / \alpha: \alpha, \beta \in \bar{\Delta}_{n}, 0 \leqslant \alpha \leqslant \beta \leqslant 0^{+1}\right\} \subseteq \bar{\Delta}_{n}^{\#}$.

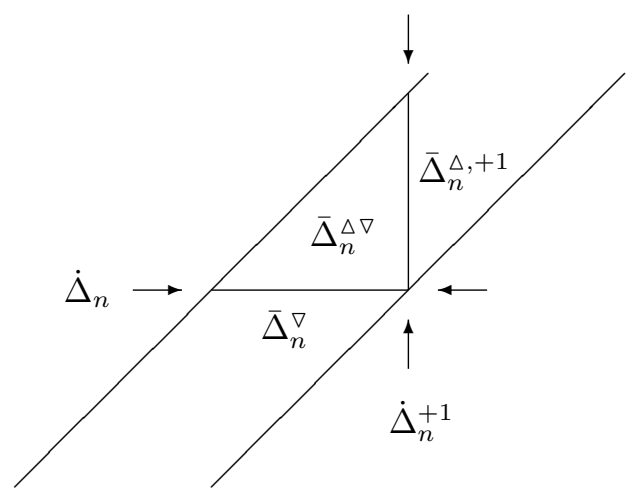

Let $\mathcal{C}^{+ \text {split }}\left(\bar{\Delta}_{n}^{\Delta \nabla}\right)$ be the full subcategory of $\mathcal{C}^{+}\left(\bar{\Delta}_{n}^{\Delta \nabla}\right)$ defined by

$$
\operatorname{ObC}^{+, \text {split }}\left(\bar{\Delta}_{n}^{\Delta \nabla}\right):=\left\{X \in \operatorname{ObC}^{+}\left(\bar{\Delta}_{n}^{\Delta \nabla}\right): \begin{array}{l}
X_{\gamma / \alpha} \stackrel{x}{\longrightarrow} X_{\delta / \beta} \text { is split } \\
\text { for all } \gamma / \alpha, \delta / \beta \in \bar{\Delta}_{n}^{\Delta \nabla} \\
\text { with } \gamma / \alpha \leqslant \delta / \beta .
\end{array}\right\}
$$

We denote the quotient category by

$$
\underline{\mathcal{C}^{+}\left(\bar{\Delta}_{n}^{\Delta \nabla}\right)}:=\mathcal{C}^{+}\left(\bar{\Delta}_{n}^{\Delta \nabla}\right) / \mathcal{C}^{+, \text {split }}\left(\bar{\Delta}_{n}^{\Delta \nabla}\right) .
$$

\section{Lemma 2.7.}

(1) The restriction functors

$$
\begin{aligned}
& \mathcal{C}^{+}\left(\bar{\Delta}_{n}^{\#}\right) \stackrel{\left.(-)\right|_{\Delta_{n} \nabla}}{\longrightarrow} \mathcal{C}^{+}\left(\bar{\Delta}_{n}^{\Delta \nabla}\right) \stackrel{\left.(-)\right|_{\dot{\Delta}_{n}}}{\longrightarrow} \mathcal{C}^{+}\left(\dot{\Delta}_{n}\right) \\
& \left.X \longmapsto X\right|_{\bar{\Delta}_{n}^{\Delta \nabla}} \\
& \left.Y \longmapsto Y\right|_{\dot{\Delta}_{n}}
\end{aligned}
$$

are full and strictly dense.

(2) The functors

$$
\begin{aligned}
\left.\frac{\mathcal{C}^{+}\left(\bar{\Delta}_{n}^{\#}\right)}{X} \stackrel{\left.(-)\right|_{\bar{\Delta}_{n}^{\Delta \nabla}}}{\longmapsto} \frac{\mathcal{C}^{+}\left(\bar{\Delta}_{n}^{\Delta \nabla}\right)}{\left.X\right|_{\bar{\Delta}_{n}^{\Delta \nabla}}} \stackrel{\left.(-)\right|_{\Delta_{n}}}{\longrightarrow} \stackrel{\mathcal{C}^{+}\left(\dot{\Delta}_{n}\right)}{Y} \longmapsto Y\right|_{\dot{\Delta}_{n}},
\end{aligned}
$$

induced by restriction, are equivalences. 
Proof. Ad (1). The composition

$$
\begin{aligned}
\mathcal{C}^{+}\left(\bar{\Delta}_{n}^{\#}\right) & \longrightarrow \mathcal{C}^{+}\left(\bar{\Delta}_{n}^{\Delta \nabla}\right) \longrightarrow \mathcal{C}\left(\dot{\Delta}_{n}\right) \\
X & \left.\left.\longmapsto X\right|_{\bar{\Delta}_{n}^{\Delta \nabla}} \longmapsto X\right|_{\dot{\Delta}_{n}}
\end{aligned}
$$

is strictly dense by Proposition 2.4 and full by Proposition 2.5. Therefore the restriction functor from $\mathcal{C}^{+}\left(\bar{\Delta}_{n}^{\Delta \nabla}\right)$ to $\mathcal{C}^{+}\left(\dot{\Delta}_{n}\right)$ is full and strictly dense. Let

We claim that the restriction functor from $\mathcal{C}^{+}\left(\bar{\Delta}_{n}^{\#}\right)$ to $\mathcal{C}^{+}\left(\bar{\Delta}_{n}^{\Delta \nabla}\right)$ is strictly dense.

$$
\begin{aligned}
\mathcal{C}\left(\dot{\Delta}_{n}^{+1}\right) & \stackrel{S^{\Delta,+1}}{\longrightarrow} \hat{\mathcal{C}}^{+}\left(\bar{\Delta}_{n}^{\Delta,+1}\right) \\
X & \longmapsto\left(X^{(+1)} S^{\Delta}\right)^{(-1)} ;
\end{aligned}
$$

see $\S 2.1 .4$, and similarly

$$
\begin{aligned}
\mathrm{Ob} \hat{\mathcal{C}}^{+}\left(\bar{\Delta}_{n}^{\Delta,+1}\right) & \stackrel{R^{\Delta,+1}}{\longrightarrow} \mathrm{ObC}^{+}\left(\bar{\Delta}_{n}^{\Delta,+1}\right) \\
X & \longmapsto\left(X^{(+1)} R^{\Delta}\right)^{(-1)} .
\end{aligned}
$$

Given $X \in \mathrm{ObC}^{+}\left(\bar{\Delta}_{n}^{\Delta \nabla}\right)$, we may define $X^{\prime} \in \mathrm{ObC}^{+}\left(\bar{\Delta}_{n}^{\#}\right)$ letting

$$
\begin{aligned}
\left.X^{\prime}\right|_{\bar{\Delta}_{n}^{\Delta,+1}} & :=\left.X\right|_{{\Delta_{n}^{+1}}^{+1}} S^{\Delta,+1} R^{\Delta,+1} \\
\left.X^{\prime}\right|_{\bar{\Delta}_{n}^{\Delta \nabla}} & :=X \\
\left.X^{\prime}\right|_{\bar{\Delta}_{n}^{\nabla}} & :=\left.X\right|_{\dot{\Delta}_{n}} S^{\nabla} R^{\nabla} .
\end{aligned}
$$

We claim that the restriction functor from $\bar{\Delta}_{n}^{\#}$ to $\bar{\Delta}_{n}^{\Delta \nabla}$ is full. Suppose given $X, Y \in \mathrm{ObC}^{+}\left(\Delta_{n}^{\#}\right)$ and a morphism $\left.\left.X\right|_{\bar{\Delta}_{n}^{\Delta \nabla}} \stackrel{f}{\longrightarrow} Y\right|_{\bar{\Delta}_{n}^{\Delta \nabla}}$. By Proposition 2.6 and a shift, there exists a morphism $\left.\left.X\right|_{\Delta_{n}^{\Delta,+1}} \stackrel{f^{\Delta}}{\longrightarrow} Y\right|_{\Delta_{n}^{\Delta,+1}}$ such that $\left.f^{\Delta}\right|_{\Delta_{n}^{+1}}=\left.f\right|_{\dot{\Delta}_{n}^{+1}}$. By Proposition 2.6 and by duality, there exists a morphism $\left.\left.X\right|_{\Delta_{n}^{\nabla}} \stackrel{f^{\nabla}}{\longrightarrow} Y\right|_{\Delta_{n}^{\nabla}}$ such that $\left.f^{\nabla}\right|_{\dot{\Delta}_{n}}=\left.f\right|_{\dot{\Delta}_{n}}$. We may define a morphism $X \stackrel{f^{\prime}}{\longrightarrow} Y$ letting

$$
\begin{aligned}
\left.f^{\prime}\right|_{\bar{\Delta}_{n}^{\Delta,+1}} & :=f^{\Delta} \\
\left.f^{\prime}\right|_{\bar{\Delta}_{n}^{\Delta \nabla}} & :=f \\
\left.f^{\prime}\right|_{\bar{\Delta}_{n}^{\nabla}} & :=f^{\nabla} .
\end{aligned}
$$

Ad (2). The composition

$$
\begin{aligned}
\frac{\mathcal{C}^{+}\left(\bar{\Delta}_{n}^{\#}\right)}{X} & \left.\left.\longrightarrow X\right|_{{\overline{\Delta_{n}^{\Delta}}}^{+}\left(\bar{\Delta}_{n}^{\Delta \nabla}\right)} \longmapsto X\right|_{\dot{\Delta}_{n}}
\end{aligned}
$$

is an equivalence by Proposition 2.6. Therefore the functor induced by restriction from $\bar{\Delta}_{n}^{\#}$ to $\bar{\Delta}_{n}^{\Delta \nabla}$ is faithful. By (1), it is full and dense, and so it is an equivalence. Therefore the functor induced by restriction from $\bar{\Delta}_{n}^{\Delta \nabla}$ to $\dot{\Delta}_{n}$ is an equivalence also.

\subsubsection{CUtTing OFF THE LAST OBJECT}

Putting $n=2$, the equivalence given in Lemma 2.10, when composed with the equivalence in Proposition 2.6, can be used to retrieve HELLER's original isomorphism, called $\delta(\Delta)$ in $[\mathbf{1 2}$, p. 53]. 
In this section, we suppose that $n \geqslant 2$. Consider the functor

$$
\begin{aligned}
\mathcal{C}\left(\dot{\Delta}_{n}\right) & \stackrel{K}{\longrightarrow} \hat{\mathcal{C}}\left(\dot{\Delta}_{n-1}\right) \\
X & \longmapsto X K:=\left.\left(X \uparrow_{0}\right)\right|_{\dot{\Delta}_{n-1}},
\end{aligned}
$$

where 0 denotes the morphism $0 \stackrel{0}{\longrightarrow} X_{n}$ (see $\left.\S \mathrm{A} .7\right)$.

Explicitly, we have $(X K)_{i}:=\operatorname{Kern}\left(X_{i} \stackrel{x}{\longrightarrow} X_{n}\right)$ for $i \in[1, n-1]$, taken in $\hat{\mathcal{C}}$, equipped with the induced morphisms $(X K)_{i} \longrightarrow(X K)_{j}$ for $i, j \in[1, n-1]$ with $i \leqslant j$, fitting into a pullback $\left((X K)_{i},(X K)_{j}, X_{i}, X_{j}\right)$.

Let $\mathcal{C}^{\text {split }}\left(\dot{\Delta}_{n}\right) \subseteq \mathcal{C}\left(\dot{\Delta}_{n}\right)$ be the full subcategory defined by

$$
\operatorname{Ob} \mathcal{C}^{\text {split }}\left(\dot{\Delta}_{n}\right):=\left\{X \in \operatorname{ObC}\left(\dot{\Delta}_{n}\right): \begin{array}{l}
\left(X_{i} \longrightarrow X_{j}\right) \text { is split } \\
\text { for all } i, j \in[1, n] \text { with } i \leqslant j
\end{array}\right\},
$$

and let $\mathcal{C}\left(\dot{\Delta}_{n}\right):=\mathcal{C}\left(\dot{\Delta}_{n}\right) / \mathcal{C}^{\text {split }}\left(\dot{\Delta}_{n}\right)$ and $\hat{\mathcal{C}}\left(\dot{\Delta}_{n-1}\right):=\hat{\mathcal{C}}\left(\dot{\Delta}_{n-1}\right) / \mathcal{C}^{\text {split }}\left(\dot{\Delta}_{n-1}\right)$.

For $\overline{Y \in \mathrm{Ob}} \hat{\mathcal{C}}\left(\dot{\Delta}_{n}\right)$ and $i \in[1, n]$, we let $Y R_{i}:=Y \uparrow Y_{i \iota} \in \mathrm{Ob} \hat{\mathcal{C}}\left(\dot{\Delta}_{n}\right)$.

We have a resolution map

$$
\begin{aligned}
\operatorname{Ob} \hat{\mathcal{C}}\left(\dot{\Delta}_{n}\right) & \stackrel{R_{n}^{\prime}}{\longrightarrow} \operatorname{Ob} \mathcal{C}\left(\dot{\Delta}_{n}\right) \\
Y & \longmapsto Y R_{n}^{\prime}:=Y R_{0} \cdots R_{n}
\end{aligned}
$$

If $Y \in \mathrm{Ob} \hat{\mathcal{C}}\left(\dot{\Delta}_{n}\right)$ consists of monomorphisms, then so does $Y R_{n}^{\prime}$, from which $Y R_{n}^{\prime} \in$ $\operatorname{Ob} \mathcal{C}^{\operatorname{split}}\left(\dot{\Delta}_{n}\right)$

Given a morphism $Y \longrightarrow Y^{\prime}$ in $\hat{\mathcal{C}}\left(\dot{\Delta}_{n}\right)$ with $Y^{\prime}$ having bijective entries, this morphism factors over $Y \longrightarrow Y R_{n}^{\prime}$ by injectivity of the entries of $Y^{\prime}$ and by the universal property of the pointwise pushout.

Lemma 2.8. The functor $K$ is dense.

Proof. Suppose given $X \in \mathrm{Ob} \hat{\mathcal{C}}\left(\dot{\Delta}_{n-1}\right)$. Let $X^{\prime} \in \mathrm{Ob} \hat{\mathcal{C}}\left(\dot{\Delta}_{n}\right)$ be defined by $\left.X^{\prime}\right|_{\dot{\Delta}_{n-1}}:=X$ and $X_{n}^{\prime}:=0$. Then $X^{\prime} R_{n}^{\prime} \in \operatorname{ObC}\left(\dot{\Delta}_{n}\right)$ has $\left(X^{\prime} R_{n}^{\prime}\right) K \simeq X$.

Lemma 2.9. The functor $K$ is full.

Proof. Suppose given $X, Y \in \mathrm{ObC}\left(\dot{\Delta}_{n}\right)$ and a morphism $X K \stackrel{f}{\longrightarrow} Y K$. We claim that there exists a morphism $X \stackrel{\tilde{f}}{\longrightarrow} Y$ such that $\tilde{f} K=f$. We construct its components $\tilde{f}_{l}$ by induction on $l$. For $l=1$, we obtain a morphism $X_{1} \stackrel{\tilde{f}_{1}}{\longrightarrow} Y_{1}$ such that $\left((X K)_{1},(Y K)_{1}, X_{1}, Y_{1}\right)$ commutes by injectivity of $Y_{1}$ in $\hat{\mathcal{C}}$. For $l \geqslant 2$, we obtain a morphism $X_{l} \stackrel{\tilde{f}_{l}}{\longrightarrow} Y_{l}$ such that $\left((X K)_{l},(Y K)_{l}, X_{l}, Y_{l}\right)$ and $\left(X_{l-1}, Y_{l-1}, X_{l}, Y_{l}\right)$ commute, by the fact that $\left((X K)_{l-1},(X K)_{l}, X_{l-1}, X_{l}\right)$ is a weak square and by injectivity of $Y_{l}$.

Proposition 2.10. The functor $K$ induces an equivalence

$$
\begin{aligned}
& \underline{\mathcal{C}\left(\dot{\Delta}_{n}\right)} \stackrel{\underline{K}}{\sim} \underline{\hat{\mathcal{C}}\left(\dot{\Delta}_{n-1}\right)} \\
& X \longmapsto X K \text {. }
\end{aligned}
$$


Proof. Let $\tilde{\mathcal{C}} \subseteq \hat{\mathcal{C}}$ denote the full subcategory of bijective objects in $\hat{\mathcal{C}}$. Every object in $\tilde{\mathcal{C}}$ is a direct summand of an object in $\mathcal{C}$. Let $\tilde{\mathcal{C}}^{\text {split }}\left(\dot{\Delta}_{n-1}\right) \subseteq \tilde{\mathcal{C}}\left(\dot{\Delta}_{n-1}\right)$ be the full subcategory defined by

$$
\operatorname{Ob} \tilde{\mathcal{C}}^{\text {split }}\left(\dot{\Delta}_{n-1}\right):=\left\{X \in \tilde{\mathcal{C}}\left(\dot{\Delta}_{n-1}\right): \begin{array}{ll}
\left(X_{i} \longrightarrow X_{j}\right) \text { is split for all } \\
i, j \in[1, n-1] \text { with } i \leqslant j
\end{array}\right\}
$$

Let $Y$ be an object of $\tilde{\mathcal{C}}^{\text {split }}\left(\dot{\Delta}_{n-1}\right)$. Then $Y R_{n-1}^{\prime}$ is an object of $\mathcal{C}^{\text {split }}\left(\dot{\Delta}_{n-1}\right)$ that has $Y$ as a direct summand since the identity on $Y$ factors over $Y \rightarrow Y R_{n-1}$.

Therefore any morphism that factors over an object of $\tilde{\mathcal{C}}^{\text {split }}\left(\dot{\Delta}_{n-1}\right)$ already factors over an object of $\mathcal{C}^{\text {split }}\left(\dot{\Delta}_{n-1}\right)$. We infer that

$$
\hat{\mathcal{C}\left(\dot{\Delta}_{n-1}\right)}=\hat{\mathcal{C}}\left(\dot{\Delta}_{n-1}\right) / \tilde{\mathcal{C}}^{\text {split }}\left(\dot{\Delta}_{n-1}\right) \text {. }
$$

Suppose given $X \in \mathrm{Ob} \mathcal{C}\left(\dot{\Delta}_{n}\right)$. Denote $X^{\prime}:=X K \in \mathrm{Ob} \hat{\mathcal{C}}\left(\dot{\Delta}_{n-1}\right)$.

We claim that if $X \in \mathrm{Ob} \mathcal{C}^{\text {split }}\left(\dot{\Delta}_{n}\right)$, then $X^{\prime} \in \operatorname{Ob} \tilde{\mathcal{C}}^{\text {split }}\left(\dot{\Delta}_{n-1}\right)$. First of all, $X_{i}^{\prime}$ is bijective for $i \in[1, n-1]$, since the image of $X_{i} \longrightarrow X_{n}$ is bijective, and since $X_{i}^{\prime}$ is the kernel of this morphism. Now suppose given $i, j \in[1, n-1]$ with $i<j$. Let $B$ be the image of $X_{i} \longrightarrow X_{j}$, and form a pullback $\left(B^{\prime}, X_{j}^{\prime}, B, X_{j}\right)$. Then there is an induced morphism $X_{i}^{\prime} \longrightarrow B^{\prime}$ turning $\left(X_{i}^{\prime}, B^{\prime}, X_{i}, B\right)$ into a commutative quadrangle, which is a pullback by composition to a pullback $\left(X_{i}^{\prime}, X_{j}^{\prime}, X_{i}, X_{j}\right)$. We insert the common kernel $Z$ of $X_{i} \longrightarrow X_{j}$ and $X_{i}^{\prime} \longrightarrow X_{j}^{\prime}$.

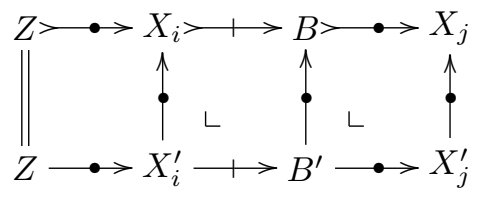

Hence $Z \longrightarrow X_{i}^{\prime}$ is split monomorphic, and therefore $X^{\prime} \longrightarrow B^{\prime}$ is split epimorphic. Thus $B^{\prime}$ is bijective, and so finally $B^{\prime} \longrightarrow X_{j}^{\prime}$ is split monomorphic. This proves the claim.

We claim that if $X^{\prime} \in \mathrm{Ob} \tilde{\mathcal{C}}^{\text {split }}\left(\dot{\Delta}_{n-1}\right)$, then $X \in \mathrm{ObC}^{\text {split }}\left(\dot{\Delta}_{n}\right)$. Suppose given $i, j \in[1, n-1]$ with $i<j$. We have to show that $X_{i} \longrightarrow X_{j}$ is split. We insert the image $B$ of $X_{i} \longrightarrow X_{j}$ and form a pullback $\left(B^{\prime}, X_{j}^{\prime}, B, X_{j}\right)$. Since $\left(X_{i}^{\prime}, B^{\prime}, X_{i}, B\right)$ is a square, and since $X_{i}^{\prime}$ is bijective, its diagonal sequence is split short exact. Hence $B$ is bijective as a direct summand of $X_{i} \oplus B^{\prime}$.

Invoking Lemma A.1 to prove the equivalence, it remains to show that given $X \stackrel{f}{\longrightarrow} Y$ in $\mathcal{C}\left(\dot{\Delta}_{n}\right)$ such that $f K=0$, there exists an object $V$ in $\mathcal{C}^{\text {split }}\left(\dot{\Delta}_{n}\right)$ such that there exists a factorisation

$$
(X \stackrel{f}{\longrightarrow} Y)=(X \longrightarrow V \longrightarrow Y) .
$$

Denote by $X K^{\prime} \in \operatorname{ObC}\left(\dot{\Delta}_{n}\right)$ the object that restricts to $X K$ on $\dot{\Delta}_{n-1}$ and that has $\left(X K^{\prime}\right)_{n}:=0$. Let $U$ be the cokernel of $X K^{\prime} \rightarrow X$ and consider the following 
diagram.

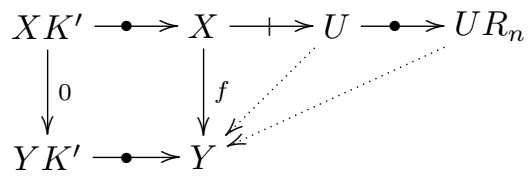

The morphism $U \longrightarrow Y$ is induced by the universal property of the cokernel. Its factorisation $(U \longrightarrow Y)=\left(U \rightarrow U R_{n} \longrightarrow Y\right)$ exists since $Y$ consists of bijective objects.

Since the morphism $X K^{\prime} \rightarrow X$ consists of pullbacks, its cokernel $U$ consists of monomorphisms. Hence so does $V:=U R_{n}$, which is therefore in $\operatorname{Ob}^{\text {split }}\left(\dot{\Delta}_{n}\right)$, as required.

\subsubsection{Not QUiTe AN EQUiVAlence}

Let $\mathcal{C}^{+ \text {,periodic }}\left(\bar{\Delta}_{n}^{\#}\right)$ be the subcategory of $\mathcal{C}^{+}\left(\bar{\Delta}_{n}^{\#}\right)$ that consists of morphisms $X \stackrel{f}{\longrightarrow} Y$ for which

$$
\left(\left[X^{+1}\right] \stackrel{\left[f^{+1}\right]}{\longrightarrow}\left[Y^{+1}\right]\right)=\left([X]^{+1} \stackrel{[f]^{+1}}{\longrightarrow}[Y]^{+1}\right) ;
$$

which is in general not a full subcategory. The objects $\mathcal{C}^{+, \text {periodic }}\left(\bar{\Delta}_{n}^{\#}\right)$ are called periodic $n$-pretriangles, the morphisms are called periodic morphisms of periodic $n$-pretriangles. Let $\mathcal{C}^{+ \text {,split,periodic }}\left(\bar{\Delta}_{n}^{\#}\right):=\mathcal{C}^{+, \text {periodic }}\left(\bar{\Delta}_{n}^{\#}\right) \cap \mathcal{C}^{+ \text {,split }}\left(\bar{\Delta}_{n}^{\#}\right)$.

For instance, if $(\mathcal{C}, T, \vartheta)$ is a Heller triangulated category, then

$$
\mathcal{C}^{+, \vartheta=1}\left(\bar{\Delta}_{n}^{\#}\right) \subseteq \mathcal{C}^{+, \text {periodic }}\left(\bar{\Delta}_{n}^{\#}\right)
$$

is a full subcategory.

For $Y \in \mathrm{Ob} \mathcal{C}\left(\dot{\Delta}_{n}\right)$, we define $Y S \in \mathrm{Ob} \mathcal{C}^{+}\left(\bar{\Delta}_{n}^{\#}\right)$ by

$$
\begin{aligned}
\left.Y S\right|_{\bar{\Delta}_{n}^{\Delta}} & :=Y S^{\Delta} \\
\left.Y S\right|_{\bar{\Delta}_{n}^{\nabla}} & :=Y S^{\nabla}
\end{aligned}
$$

and similarly on morphisms. If $Y \in \mathrm{Ob}^{\text {split }}\left(\dot{\Delta}_{n}\right)$, then $Y S \in \mathrm{Ob}^{+, \text {split }}\left(\bar{\Delta}_{n}^{\#}\right)$ by Lemma 1.4, since $\left.Y S\right|_{\dot{\Delta}_{n}}=Y$ is split.

To any $X \in \mathrm{ObC}^{+}\left(\bar{\Delta}_{n}^{\#}\right)$ for which $X_{\beta / \alpha}$ is zero for all but finitely many $\beta / \alpha \in$ $\bar{\Delta}_{n}^{\#}$ we can assign its periodification

$$
\bar{X}:=\bigoplus_{i \in \mathbf{Z}}\left[X^{+i}\right]^{-i} \in \operatorname{Ob} \mathcal{C}^{+, \text {periodic }}\left(\bar{\Delta}_{n}^{\#}\right),
$$

and similarly for morphisms between such objects. If $X$ is split, so is $\bar{X}$.

We have the restriction functor

$$
\begin{aligned}
\mathcal{C}^{+ \text {,split,periodic }}\left(\bar{\Delta}_{n}^{\#}\right) \stackrel{\left.(-)\right|_{\dot{\Delta}_{n}}}{\longrightarrow} \mathcal{C}^{\text {split }}\left(\dot{\Delta}_{n}\right) \\
\left.X \longmapsto X\right|_{\dot{\Delta}_{n}}
\end{aligned}
$$

which is not faithful in general, as the case $n=2$ shows. In the inverse direction, 
we dispose of the functor

$$
\begin{gathered}
\mathcal{C}^{+, \text {split,periodic }}\left(\bar{\Delta}_{n}^{\#}\right) \longleftarrow{ }^{\bar{S}} \mathcal{C}^{\text {split }}\left(\dot{\Delta}_{n}\right) \\
\overline{Y S}=: Y \bar{S} \longleftarrow Y .
\end{gathered}
$$

Lemma 2.11. For $X \in \operatorname{Ob} \mathcal{C}^{+ \text {,split,periodic }}\left(\bar{\Delta}_{n}^{\#}\right)$, we have $\left.X \simeq X\right|_{\dot{\Delta}_{n}} \bar{S}$.

Note that we do not claim that $\left.1 \simeq(-)\right|_{\dot{\Delta}_{n}} \bar{S}$ as endofunctors of $\mathcal{C}^{+, \text {split,periodic }}\left(\bar{\Delta}_{n}^{\#}\right)$.

Proof. We have a short exact sequence

$$
\left.\left.\left.\left.X\right|_{\dot{\Delta}_{n}} S\right|_{\bar{\Delta}_{n}^{\Delta \nabla}} \longrightarrow X\right|_{\bar{\Delta}_{n}^{\Delta \nabla}} \longrightarrow\left[\left.X\right|_{\dot{\Delta}_{n}} S^{+1}\right]^{-1}\right|_{\bar{\Delta}_{n}^{\Delta \nabla}}
$$

in $\hat{\mathcal{C}}\left(\bar{\Delta}_{n}^{\Delta \nabla}\right)$, and it suffices to show that it splits. Write $C:=\left.\left.X\right|_{\dot{\Delta}_{n}} S\right|_{\bar{\Delta}_{n}^{\Delta \nabla}}$.

It suffices to show that there exists a retraction to $\left.C \longrightarrow X\right|_{\bar{\Delta}_{n}^{\Delta \nabla}}$, which we will construct by induction. Suppose given $0<\alpha<\beta \leqslant 0^{+1}$. We may assume that after restriction of $\left.C \longrightarrow X\right|_{\bar{\Delta}_{n}^{\Delta \nabla}}$ to $\left\{\delta / \gamma \in \bar{\Delta}_{n}^{\Delta \nabla}: \delta / \gamma<\beta / \alpha\right\}$, there exists a retraction. Let $\alpha^{\prime}:=\alpha-1$ be the predecessor of $\alpha$, and let $\beta^{\prime}:=\beta-1$ be the predecessor of $\beta$, using that $\bar{\Delta}_{n}$ is linearly ordered. It suffices to show that the morphism from the quadrangle $\left(C_{\beta^{\prime} / \alpha^{\prime}}, C_{\beta^{\prime} / \alpha}, C_{\beta / \alpha^{\prime}}, C_{\beta / \alpha}\right)$ to the quadrangle $\left(X_{\beta^{\prime} / \alpha^{\prime}}, X_{\beta^{\prime} / \alpha}, X_{\beta / \alpha^{\prime}}\right.$, $\left.X_{\beta / \alpha}\right)$ has a retraction.

Let $\left(X_{\beta^{\prime} / \alpha^{\prime}}, X_{\beta^{\prime} / \alpha}, X_{\beta / \alpha^{\prime}}, T\right)$ be the pushout in $\hat{\mathcal{C}}$. The quadrangle $\left(C_{\beta^{\prime} / \alpha^{\prime}}, C_{\beta^{\prime} / \alpha}\right.$, $\left.C_{\beta / \alpha^{\prime}}, C_{\beta / \alpha}\right)$ is a pushout. The induced morphism from $\left(C_{\beta^{\prime} / \alpha^{\prime}}, C_{\beta^{\prime} / \alpha}, C_{\beta / \alpha^{\prime}}, C_{\beta / \alpha}\right)$ to $\left(X_{\beta^{\prime} / \alpha^{\prime}}, X_{\beta^{\prime} / \alpha}, X_{\beta / \alpha^{\prime}}, T\right)$ has a retraction by functoriality of the pushout. The morphism $T \longrightarrow X_{\beta / \alpha}$ induced by pushout is a monomorphism in $\hat{\mathcal{C}}$, since $\left(X_{\beta^{\prime} / \alpha^{\prime}}\right.$, $\left.X_{\beta^{\prime} / \alpha}, X_{\beta / \alpha^{\prime}}, X_{\beta / \alpha}\right)$ is a weak square. Note that $\left(C_{\beta / \alpha}, T, X_{\beta / \alpha}\right)$ is a commutative triangle.

The morphism $T \longrightarrow C_{\beta / \alpha}$ that is part of the retraction of quadrangles factors as

$$
\left(T \longrightarrow C_{\beta / \alpha}\right)=\left(T \rightarrow X_{\beta / \alpha} \longrightarrow C_{\beta / \alpha}\right),
$$

since $C_{\beta / \alpha}$ is injective in $\hat{\mathcal{C}}$ as a summand of $C_{\beta / 0}=X_{\beta / 0}$. Now $X_{\beta / \alpha} \longrightarrow C_{\beta / \alpha}$ completes the three morphisms on the other vertices to a retraction of quadrangles from $\left(X_{\beta^{\prime} / \alpha^{\prime}}, X_{\beta^{\prime} / \alpha}, X_{\beta / \alpha^{\prime}}, X_{\beta / \alpha}\right)$ to $\left(C_{\beta^{\prime} / \alpha^{\prime}}, C_{\beta^{\prime} / \alpha}, C_{\beta / \alpha^{\prime}}, C_{\beta / \alpha}\right)$ as sought.

\section{Verification of Verdier's axioms}

Let $(\mathcal{C}, \mathrm{T}, \vartheta)$ be a Heller triangulated category.

\subsection{Restriction from $\mathcal{C}^{+, \vartheta=1}\left(\bar{\Delta}_{n}^{\#}\right)$ to $\mathcal{C}\left(\dot{\Delta}_{n}\right)$ is dense and full}

Let $n \geqslant 1$. Let

$$
\begin{aligned}
\mathcal{C}\left(\dot{\Delta}_{n}^{+1}\right) & \stackrel{S^{\prime \nabla}}{\longrightarrow} \hat{\mathcal{C}}^{+}\left(\bar{\Delta}_{n}^{\nabla,+1}\right) \\
U & \longmapsto U S^{\prime \nabla}:=\left(U^{(+1)} S^{\nabla}\right)^{(-1)}
\end{aligned}
$$


Homology, Homotopy and Applications, vol. 9(2), 2007

be the conjugate by reindexing, i.e. a "shifted version" of $S^{\nabla}$; see $§ 2.1 .4$. Note that

$$
\left(U S^{\prime \nabla}\right)_{\beta / \alpha}=\operatorname{Kern}\left(U_{0^{+1} / \alpha} \stackrel{u}{\longrightarrow} U_{0^{+1} / \beta}\right)
$$

for $\alpha, \beta \in \bar{\Delta}_{n}$ with $0 \leqslant \alpha \leqslant \beta \leqslant 0^{+1}$.

Lemma 3.1. Suppose that idempotents split in $\mathcal{C}$. Given $X \in \mathrm{Ob} \mathcal{C}\left(\dot{\Delta}_{n}\right)$, there exists an $n$-triangle $\tilde{X} \in \mathrm{ObC}^{+, \vartheta=1}\left(\bar{\Delta}_{n}^{\#}\right)$ that restricts to

$$
\left.\tilde{X}\right|_{\dot{\Delta}_{n}}=X \text {. }
$$

In other words, the restriction functor $\mathcal{C}^{+, \vartheta=1}\left(\bar{\Delta}_{n}^{\#}\right) \stackrel{\left.(-)\right|_{\Delta_{n}}}{\longrightarrow} \mathcal{C}\left(\dot{\Delta}_{n}\right)$ is strictly dense.

Proof. Let $Y:=X R \in \mathrm{ObC}^{+}\left(\bar{\Delta}_{n}^{\#}\right)$; see $\S 2.2 .2 .3$. We have an isomorphism $[Y]^{+1}$ $\stackrel{Y \vartheta_{n}}{\sim}\left[Y^{+1}\right]$ in $\mathcal{C}^{+}\left(\bar{\Delta}_{n}^{\#}\right)$. Let $\left[Y^{+1}\right] \stackrel{\theta}{\longrightarrow}[Y]^{+1}$ be a representative in $\mathcal{C}^{+}\left(\bar{\Delta}_{n}^{\#}\right)$ of the inverse isomorphism $\left(Y \vartheta_{n}\right)^{-}$in $\mathcal{C}^{+}\left(\bar{\Delta}_{n}^{\#}\right)$. Consider the morphism ${ }^{6}$

$$
\left.\left.\left[Y^{+1}\right]^{-1}\right|_{\dot{\Delta}_{n}^{+1}} \stackrel{\left.[\theta]^{-1}\right|_{\Delta_{n}^{+1}}}{\longrightarrow}[Y]^{+1-1}\right|_{\dot{\Delta}_{n}^{+1}}=\left.Y\right|_{\dot{\Delta}_{n}^{+1}}
$$

We have an induced pointwise epimorphism

$$
\left.\left.\left.Y\right|_{\bar{\Delta}_{n}^{\Delta \nabla}} \longrightarrow Y\right|_{\dot{\Delta}_{n}^{+1}} S^{\prime \nabla \nabla}\right|_{\bar{\Delta}_{n}^{\Delta \nabla}}
$$

which we may use to form the pullback

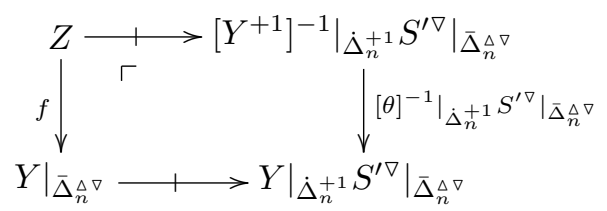

in the abelian category $\hat{\mathcal{C}}\left(\bar{\Delta}_{n}^{\Delta \nabla}\right)$, i.e. pointwise. An application of Lemma A.21.(2) to the diagonal sequence of this pullback shows that $Z \in \mathrm{Ob} \hat{\mathcal{C}}^{+, *}\left(\bar{\Delta}_{n}^{\Delta \nabla}\right)$. We obtain $Z_{\alpha / \alpha}=0$ for all $0 \leqslant \alpha \leqslant 0^{+1}$; and we obtain $Z_{0^{+1} / 0}=0$. Hence we have $Z \in$ $\operatorname{Ob} \hat{\mathcal{C}}^{+}\left(\bar{\Delta}_{n}^{\Delta \nabla}\right)$.

Suppose given $\beta / \alpha \in \bar{\Delta}_{n}$. We claim that $Z_{\beta / \alpha} \stackrel{f_{\beta / \alpha}}{\longrightarrow} Y_{\beta / \alpha}$ represents an isomorphism in $\hat{\mathcal{C}} / \mathcal{C}$. By Lemma A.24, it suffices to show that

$$
\left(\left.\left[Y^{+1}\right]^{-1}\right|_{\dot{\Delta}_{n}^{+1}} S^{\prime \nabla}\right)_{\beta / \alpha} \stackrel{\left(\left.[\theta]^{-1}\right|_{\dot{\Delta}_{n}^{+1}} S^{\prime \nabla}\right)_{\beta / \alpha}}{\longrightarrow}\left(\left.Y\right|_{\dot{\Delta}_{n}^{+1}} S^{\prime \nabla}\right)_{\beta / \alpha}
$$

represents an isomorphism in $\hat{\mathcal{C}} / \mathcal{C}$. Since evaluation at $\beta / \alpha$ induces a functor from $\hat{\mathcal{C}}^{+}\left(\bar{\Delta}_{n}^{\Delta \nabla}\right) / \mathcal{C}^{+, \text {split }}\left(\bar{\Delta}_{n}^{\Delta \nabla}\right)$ to $\hat{\mathcal{C}} / \mathcal{C}$, where $\mathcal{C}^{+, \text {split }}\left(\bar{\Delta}_{n}^{\Delta \nabla}\right)$ denotes the full subcategory of $\mathcal{C}^{+}\left(\bar{\Delta}_{n}^{\Delta \nabla}\right)$ consisting of diagrams all of whose morphisms split, it suffices to show that

$$
\left.\left.\left.\left.\left[Y^{+1}\right]^{-1}\right|_{\dot{\Delta}_{n}^{+1}} S^{\prime \nabla}\right|_{\bar{\Delta}_{n}^{\Delta \nabla}} \stackrel{\left.\left.[\theta]^{-1}\right|_{\Delta_{n}^{+1}} S^{\prime \nabla}\right|_{\bar{\Delta}_{n}^{\Delta \nabla}}}{\longrightarrow} Y\right|_{\dot{\Delta}_{n}^{+1}} S^{\prime \nabla}\right|_{\bar{\Delta}_{n}^{\Delta \nabla}}
$$

represents an isomorphism in $\hat{\mathcal{C}}^{+}\left(\bar{\Delta}_{n}^{\Delta \nabla}\right) / \mathcal{C}^{+, \text {split }}\left(\bar{\Delta}_{n}^{\Delta \nabla}\right)$.

${ }^{6}$ We recall the convention that the inverse of the outer shift applied to a morphism $f$ is written $[f]^{-1}$, whereas $f^{-}$denotes the inverse morphism, if existent. 
Now $\left.(-)^{(-1)} S^{\prime \nabla}\right|_{\bar{\Delta}_{n}^{\Delta \nabla}}$ induces a functor from $\underline{\mathcal{C}\left(\dot{\Delta}_{n}\right)}$ to $\hat{\mathcal{C}}^{+}\left(\bar{\Delta}_{n}^{\Delta \nabla}\right) / \mathcal{C}^{+, \text {split }}\left(\bar{\Delta}_{n}^{\Delta \nabla}\right)$, since it maps $\mathcal{C}^{\text {split }}\left(\dot{\Delta}_{n}\right)$ to $\mathcal{C}^{+ \text {split }}\left(\bar{\Delta}_{n}^{\Delta \nabla}\right)$ by Lemma 1.4 . Therefore, it suffices to show that

$$
\left(\left.\left[Y^{+1}\right]^{-1}\right|_{\dot{\Delta}_{n}^{+1}}\right)^{(+1)} \stackrel{\left(\left.[\theta]^{-1}\right|_{\dot{\Delta}_{n}^{+1}}\right)^{(+1)}}{\longrightarrow}\left(\left.Y\right|_{\dot{\Delta}_{n}^{+1}}\right)^{(+1)}
$$

represents an isomorphism in $\underline{\mathcal{C}\left(\dot{\Delta}_{n}\right)}$. Since $\left(\left.[-]^{-1}\right|_{\dot{\Delta}_{n}^{+1}}\right)^{(+1)}=\left.(-)\right|_{\dot{\Delta}_{n}}$, this means that it suffices to show that

$$
\left.\left.\left[Y^{+1}\right]\right|_{\dot{\Delta}_{n}} \stackrel{\left.\theta\right|_{\dot{\Delta}_{n}}}{\longrightarrow}[Y]^{+1}\right|_{\dot{\Delta}_{n}}
$$

represents an isomorphism in $\underline{\mathcal{C}\left(\dot{\Delta}_{n}\right)}$. Since $\left.(-)\right|_{\dot{\Delta}_{n}}$ induces a functor from $\underline{\mathcal{C}^{+}\left(\Delta_{n}^{\#}\right)}$ to $\underline{\mathcal{C}\left(\dot{\Delta}_{n}\right)}$, it suffices to show that

$$
\left[Y^{+1}\right] \stackrel{\theta}{\longrightarrow}[Y]^{+1}
$$

represents an isomorphism in $\underline{\mathcal{C}^{+}\left(\bar{\Delta}_{n}^{\#}\right)}$. This, however, follows by choice of $\theta$. This proves the claim.

Since idempotents are assumed to split in $\mathcal{C}$, we can conclude that for all $\beta / \alpha$ in $\bar{\Delta}_{n}^{\Delta \nabla}$, the entry $Z_{\beta / \alpha}$ is in $\mathcal{C}$. So $Z \in \operatorname{Ob}^{+}\left(\bar{\Delta}_{n}^{\Delta \nabla}\right)$.

We remark that

$$
\begin{aligned}
\left.Z\right|_{\dot{\Delta}_{n}} & =\left.Y\right|_{\dot{\Delta}_{n}} \quad=X \\
\left.Z\right|_{\dot{\Delta}_{n}^{+1}}=\left.\left[Y^{+1}\right]^{-1}\right|_{\dot{\Delta}_{n}^{+1}} & =\left(X^{+1}\right)^{(-1)},
\end{aligned}
$$

where $X^{+1}$ arises from $X$ by pointwise application of $(-)^{+1}$. Concerning morphisms, we remark that

$$
\begin{aligned}
& \left.\left(\left.Z \stackrel{f}{\longrightarrow} Y\right|_{\bar{\Delta}_{n}^{\Delta \nabla}}\right)\right|_{\dot{\Delta}_{n}}=\left(X \stackrel{1_{X}}{\longrightarrow} X\right) \\
& \left.\left(\left.Z \stackrel{f}{\longrightarrow} Y\right|_{\bar{\Delta}_{n}^{\Delta} \nabla}\right)\right|_{\dot{\Delta}_{n}^{+1}}=\left.\left(\left[Y^{+1}\right]^{-1} \stackrel{[\theta]^{-1}}{\longrightarrow} Y\right)\right|_{\dot{\Delta}_{n}^{+1}} .
\end{aligned}
$$

In fact, on $\dot{\Delta}_{n}$, the right hand side column of our pullback vanishes; and on $\dot{\Delta}_{n}^{+1}$, the lower row of our pullback is an identity.

Now, (*) allows the periodic prolongation $\bar{Z} \in \mathrm{ObC}^{+}\left(\bar{\Delta}_{n}^{\#}\right)$ of $Z \in \mathrm{ObC}^{+}\left(\bar{\Delta}_{n}^{\Delta \nabla}\right)$ to be defined by $\left.\bar{Z}\right|_{\bar{\Delta}_{n}^{\Delta \nabla}}:=Z$ and by the requirement that $[\bar{Z}]^{+1}=\left[\bar{Z}^{+1}\right]$.

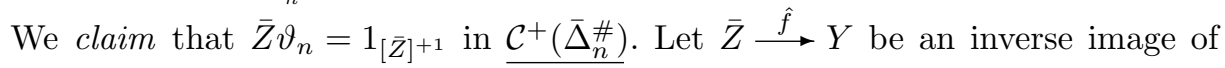
$\left.Z \stackrel{f}{\longrightarrow} Y\right|_{\bar{\Delta}_{n}^{\Delta \nabla}}$ under $\mathcal{C}^{+}\left(\bar{\Delta}_{n}^{\#}\right) \stackrel{\left.(-)\right|_{\bar{\Delta}_{n}^{\Delta} \nabla}}{\longrightarrow} \mathcal{C}^{+}\left(\bar{\Delta}_{n}^{\Delta \nabla}\right)$; see Lemma 2.7.(1). By (**), we get

$$
\begin{aligned}
& \left.(\bar{Z} \stackrel{\hat{f}}{\longrightarrow} Y)\right|_{\dot{\Delta}_{n}}=\left(X \stackrel{1_{X}}{\longrightarrow} X\right) \\
& \left.(\bar{Z} \stackrel{\hat{f}}{\longrightarrow} Y)\right|_{\dot{\Delta}_{n}^{+1}}=\left.\left(\left[Y^{+1}\right]^{-1} \stackrel{[\theta]^{-1}}{\longrightarrow} Y\right)\right|_{\dot{\Delta}_{n}^{+1}} .
\end{aligned}
$$

We consider the commutative quadrangle

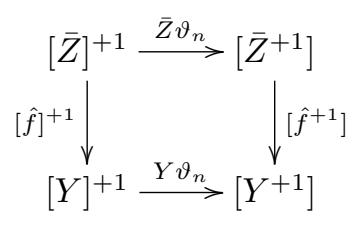


in $\underline{\mathcal{C}^{+}\left(\bar{\Delta}_{n}^{\#}\right)}$. We restrict it to $\dot{\Delta}_{n}$ to obtain the commutative quadrangle

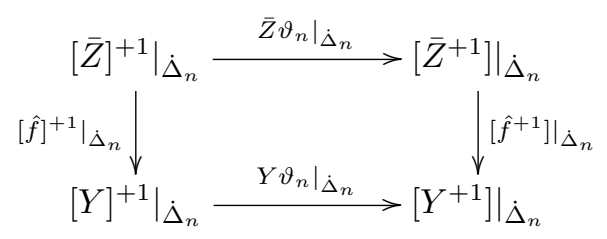

in $\underline{\mathcal{C}}\left(\dot{\Delta}_{n}\right)$, which, using $\left(* *^{\prime}\right)$, can be rewritten as

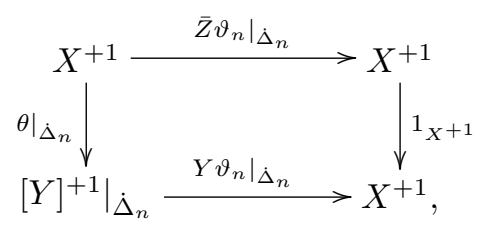

where we have not distinguished in notation between $\left.\theta\right|_{\dot{\Delta}_{n}}$ and its residue class in $\underline{\mathcal{C}\left(\dot{\Delta}_{n}\right)}$, etc.

Since $\theta\left(Y \vartheta_{n}\right)=1_{\left[Y^{+1}\right]}$ in $\underline{\mathcal{C}^{+}\left(\bar{\Delta}_{n}^{\#}\right)}$, we have $\left.\theta\right|_{\dot{\Delta}_{n}}\left(\left.Y \vartheta_{n}\right|_{\dot{\Delta}_{n}}\right)=1_{X^{+1}}$ in $\underline{\mathcal{C}\left(\dot{\Delta}_{n}\right)}$. Thus the last quadrangle shows that $\left.\bar{Z} \vartheta_{n}\right|_{\dot{\Delta}_{n}}=1_{X^{+1}}=\left.1_{[\bar{Z}]^{+1}}\right|_{\dot{\Delta}_{n}}$ in $\underline{\mathcal{C}\left(\dot{\Delta}_{n}\right)}$ as well.

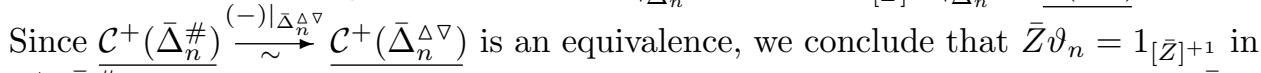
$\mathcal{C}^{+}\left(\bar{\Delta}_{n}^{\#}\right)$; see Proposition 2.6. This proves the claim; i.e. we have shown that $\bar{Z}$ is an $n$-triangle.

Since $\left.\bar{Z}\right|_{\dot{\Delta}_{n}}=X$ by $(*)$, this proves the lemma.

Note that in the proof of Lemma 3.1, we needed the assumption that idempotents split in $\mathcal{C}$ in the equivalent form that the residue class functor $\hat{\mathcal{C}} \longrightarrow \hat{\mathcal{C}} / \mathcal{C}$ maps precisely the objects of $\mathcal{C}$ to zero - just as HELLER did at that point.

Lemma 3.2. Given n-triangles $X$ and $Y$ and a morphism

$$
\left.\left.X\right|_{\dot{\Delta}_{n}} \stackrel{f}{\longrightarrow} Y\right|_{\dot{\Delta}_{n}}
$$

in $\mathcal{C}\left(\dot{\Delta}_{n}\right)$, there exists a morphism $X \stackrel{\tilde{f}}{\longrightarrow} Y$ of n-triangles such that $\left.\tilde{f}\right|_{\dot{\Delta}_{n}}=f$. In other words, the restriction functor $\mathcal{C}^{+, \vartheta=1}\left(\bar{\Delta}_{n}^{\#}\right) \stackrel{\left.(-)\right|_{\Delta_{n}}}{\longrightarrow} \mathcal{C}\left(\dot{\Delta}_{n}\right)$ is full.

Proof. Since the restriction functor $\mathcal{C}^{+}\left(\bar{\Delta}_{n}^{\#}\right) \stackrel{\left.(-)\right|_{\dot{\Delta}_{n}}}{\longrightarrow} \mathcal{C}\left(\dot{\Delta}_{n}\right)$ is full by Proposition 2.5, we find a morphism $X \stackrel{g}{\longrightarrow} Y$ in $\mathcal{C}^{+}\left(\bar{\Delta}_{n}^{\#}\right)$ such that $\left.g\right|_{\dot{\Delta}_{n}}=f$.

Let $g$ denote the residue class of $g$ in $\mathcal{C}^{+}\left(\bar{\Delta}_{n}^{\#}\right)$. Since $\vartheta_{n}$ is a transformation, we have $[\underline{g}]^{+1}\left(Y \vartheta_{n}\right)=\left(X \vartheta_{n}\right)\left[\underline{g}^{+1}\right]$. Since $\overline{X \text { and } Y}$ are $n$-triangles, both $X \vartheta_{n}$ and $Y \vartheta_{n}$ are identities, and this equality amounts to $[g]^{+1}=\left[g^{+1}\right]$, i.e. the difference $[g]^{+1}-\left[g^{+1}\right]$ factors over an object of $\mathcal{C}^{+ \text {,split }}\left(\bar{\Delta}_{n}^{\#}\right)$. Restricting to $\dot{\Delta}_{n}$, the difference

$$
\left.\left([g]^{+1}-\left[g^{+1}\right]\right)\right|_{\dot{\Delta}_{n}}=\left(\left.g\right|_{\dot{\Delta}_{n}^{+1}}\right)^{(+1)}-f^{+1}
$$

factors over an object of $\mathcal{C}^{\text {split }}\left(\dot{\Delta}_{n}\right)$. Therefore, $\left.g\right|_{\dot{\Delta}_{n}^{+1}}-\left(f^{+1}\right)^{(-1)}$ factors over an 
object $Z$ of $\mathcal{C}^{\text {split }}\left(\dot{\Delta}_{n}^{+1}\right)$, say, as

$$
\left(\left.\left.X\right|_{\dot{\Delta}_{n}^{+1}} \stackrel{\left.g\right|_{\Delta_{n}^{+1}}-\left(f^{+1}\right)^{(-1)}}{\longrightarrow} Y\right|_{\dot{\Delta}_{n}^{+1}}\right)=\left(\left.\left.X\right|_{\dot{\Delta}_{n}^{+1}} \stackrel{a}{\longrightarrow} Z \stackrel{b}{\longrightarrow} Y\right|_{\dot{\Delta}_{n}^{+1}}\right) .
$$

By periodic continuation, it suffices to find a morphism $\left.\left.X\right|_{\bar{\Delta}_{n}^{\Delta \nabla}} \stackrel{\breve{f}}{\longrightarrow} Y\right|_{\bar{\Delta}_{n}^{\Delta \nabla}}$ in $\mathcal{C}^{+}\left(\bar{\Delta}_{n}^{\Delta \nabla}\right)$ such that $\left.\breve{f}\right|_{\dot{\Delta}_{n}}=f$ and such that $\left.\breve{f}\right|_{\dot{\Delta}_{n}^{+1}}=\left(f^{+1}\right)^{(-1)}$. That is, we have to find a morphism $\left.\left.X\right|_{\bar{\Delta}_{n}^{\Delta \nabla}} \stackrel{h}{\longrightarrow} Y\right|_{\bar{\Delta}_{n}^{\Delta \nabla}}$ such that $\left.h\right|_{\dot{\Delta}_{n}}=0$ and such that $\left.h\right|_{\dot{\Delta}_{n}^{+1}}=a b$, for then we may take $\breve{f}:=\left.g\right|_{\bar{\Delta}_{n}^{\Delta \nabla}}-h$.

Note that $\left.\left(\left.Z S^{\prime \nabla}\right|_{\bar{\Delta}_{n}^{\Delta \nabla}}\right)\right|_{\dot{\Delta}_{n}}=0$. Note that $\left.Z S^{\prime \nabla}\right|_{\bar{\Delta}_{n}^{\Delta \nabla}}$ is in $\mathcal{C}^{+}\left(\bar{\Delta}_{n}^{\Delta \nabla}\right)$, hence in $\mathcal{C}^{+, \text {split }}\left(\bar{\Delta}_{n}^{\Delta \nabla}\right)$ by Lemma 1.4 .

Since $\left.S^{\prime \nabla}\right|_{\bar{\Delta}_{n}^{\Delta \nabla}}$ is right adjoint to restriction to $\dot{\Delta}_{n}^{+1}$, we have a morphism $\left.\left.X\right|_{\bar{\Delta}_{n}^{\Delta \nabla}} \stackrel{a^{\prime}}{\longrightarrow} Z S^{\prime \nabla}\right|_{\bar{\Delta}_{n}^{\Delta \nabla}}$ such that $\left.a^{\prime}\right|_{\dot{\Delta}_{n}^{+1}}=a$.

Since $\mathcal{C}^{+}\left(\bar{\Delta}_{n}^{\Delta \nabla}\right) \stackrel{(-)||_{\Delta_{n}^{+1}}}{\longrightarrow} \mathcal{C}\left(\dot{\Delta}_{n}^{+1}\right)$ is full by the dual and shifted assertion of Lemma 2.7.(1), there is a morphism $\left.\left.Z S^{\prime \nabla}\right|_{\bar{\Delta}_{n}^{\Delta \nabla}} \stackrel{b^{\prime}}{\longrightarrow} Y\right|_{\bar{\Delta}_{n}^{\Delta \nabla}}$ such that $\left.b^{\prime}\right|_{\dot{\Delta}_{n}^{+1}}=b$.

We may take $h:=a^{\prime} b^{\prime}$.

Note that in Lemmata 3.1 and 3.2, we do not claim the existence of a coretraction from $\mathcal{C}\left(\dot{\Delta}_{n}\right)$ to $\mathcal{C}^{+, \vartheta=1}\left(\bar{\Delta}_{n}^{\#}\right)$ to restriction. The construction made in the proof of Lemma 3.2 involves, for example, the choice of a lift $b^{\prime}$ of $b$. Cf. [28, II.1.2.13].

The fullness used in the proof of Lemma 3.2 to lift $b$ can also be used to lift $a$. We have used the direct argument and thus seen that the lift $a^{\prime}$ of $a$ does not involve a choice.

Remark 3.3. Suppose that idempotents split in $\mathcal{C}$. By Lemmata 3.1 and 3.2, the restriction functor

$$
\mathcal{C}^{+, \vartheta=1}\left(\bar{\Delta}_{n}^{\#}\right) \stackrel{(-) \mid \dot{\Delta}_{n}}{\longrightarrow} \mathcal{C}\left(\dot{\Delta}_{n}\right)
$$

is full and strictly dense. By Proposition 2.6, the restriction functor

$$
\underline{\mathcal{C}^{+}\left(\bar{\Delta}_{n}^{\#}\right)} \stackrel{\left.(-)\right|_{\dot{\Delta}_{n}}}{\sim} \underline{\mathcal{C}\left(\dot{\Delta}_{n}\right)}
$$

is an equivalence. Denoting by $\mathcal{C}^{+, \vartheta=1}\left(\bar{\Delta}_{n}^{\#}\right)$ the image of $\mathcal{C}^{+, \vartheta=1}\left(\bar{\Delta}_{n}^{\#}\right)$ in $\mathcal{C}^{+}\left(\bar{\Delta}_{n}^{\#}\right)$, we obtain a full and strictly dense functor $\underline{\mathcal{C}^{+, \vartheta}=1}\left(\bar{\Delta}_{n}^{\#}\right) \stackrel{\left.(-)\right|_{\dot{\Delta}_{n}}}{\longrightarrow} \underline{\mathcal{C}\left(\dot{\Delta}_{n}\right)}$. Since it factors

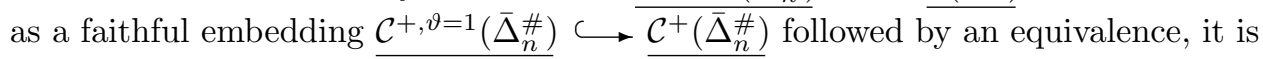
also faithful. We end up with equivalences

$$
\underline{\mathcal{C}^{+, \vartheta=1}\left(\bar{\Delta}_{n}^{\#}\right)} \stackrel{\left.(-)\right|_{\dot{\Delta}_{n}}}{\sim} \underline{\mathcal{C}\left(\dot{\Delta}_{n}\right)}, \quad \underline{\mathcal{C}^{+, \vartheta=1}\left(\bar{\Delta}_{n}^{\#}\right)} \stackrel{\sim}{\sim} \underline{\mathcal{C}^{+}\left(\bar{\Delta}_{n}^{\#}\right)} .
$$

\subsection{An omnibus lemma}

Suppose given $n, m \geqslant 1$. Concerning the category $\mathcal{C}^{+, \text {periodic }}\left(\bar{\Delta}_{n}^{\#}\right)$ of periodic $n$-pretriangles and its full subcategory $\mathcal{C}^{+ \text {,split,periodic }}\left(\bar{\Delta}_{n}^{\#}\right)$ of periodic split $n$-pretriangles, see $\S 2.5 .3$; concerning the category $\mathcal{C}^{+, \vartheta=1}\left(\bar{\Delta}_{n}^{\#}\right)$ of $n$-triangles, see Definition 1.5.(ii). Note that

$$
\mathcal{C}^{+, \vartheta=1}\left(\bar{\Delta}_{n}^{\#}\right) \subseteq \mathcal{C}^{+, \text {periodic }}\left(\bar{\Delta}_{n}^{\#}\right) \subseteq \mathcal{C}^{+}\left(\bar{\Delta}_{n}^{\#}\right)
$$


and that the first inclusion is full.

\section{Lemma 3.4.}

(1) Let $X$ be an $n$-triangle, and let $\bar{\Delta}_{n} \stackrel{p}{\longleftarrow} \bar{\Delta}_{m}$ be a morphism of periodic linearly ordered sets. Then $X p^{\#}$, obtained by "restriction along $p$ ", is an $m$-triangle.

(2) Let $X$ be a $(2 n+1)$-triangle. Then $X \mathfrak{f}_{n}$, obtained by folding, is an $(n+1)$ triangle.

(3) The category $\mathcal{C}^{+, \vartheta=1}\left(\bar{\Delta}_{n}^{\#}\right)$ of $n$-triangles is a full additive subcategory of the category $\mathcal{C}^{+ \text {,periodic }}\left(\bar{\Delta}_{n}^{\#}\right)$ of periodic $n$-pretriangles, closed under direct summands.

(4) Suppose given an isomorphism $X \stackrel{f}{\longrightarrow} Y$ in $\mathcal{C}^{+, \text {periodic }}\left(\bar{\Delta}_{n}^{\#}\right)$. If $X$ is an $n$-triangle, then $Y$ is an n-triangle.

(5) Let $X \stackrel{f}{\longrightarrow} Y$ be a morphism in $\mathcal{C}^{+ \text {,periodic }}\left(\bar{\Delta}_{n}^{\#}\right)$ such that $\left.f\right|_{\dot{\Delta}_{n}}$ is an isomorphism. Then $f$ is an isomorphism.

(6) Let $X$ and $Y$ be n-triangles. Suppose given an isomorphism $\left.\left.X\right|_{\dot{\Delta}_{n}} \stackrel{u}{\sim} Y\right|_{\dot{\Delta}_{n}}$ in $\mathcal{C}\left(\dot{\Delta}_{n}\right)$. Then there exists an isomorphism $X \stackrel{\tilde{u}}{\sim} Y$ in $\mathcal{C}^{+, \vartheta=1}\left(\bar{\Delta}_{n}^{\#}\right)$ such that $\left.\tilde{u}\right|_{\mathcal{C}\left(\dot{\Delta}_{n}\right)}=u$.

(7) If $X \in \mathrm{ObC}^{+ \text {,split,periodic }}\left(\bar{\Delta}_{n}^{\#}\right)$, then $X$ is an $n$-triangle.

Note that Lemma 3.4.(5) applies in particular to $n$-triangles and a morphism of $n$-triangles.

Proof. Ad (1). In $\underline{\mathcal{C}^{+}\left(\bar{\Delta}_{m}^{\#}\right)}$, we have

$$
\left(X \underline{p}^{\#}\right) \vartheta_{m}=\left(X \vartheta_{n}\right) \underline{p}^{\#}=\left(1_{[X]+1}\right) \underline{p}^{\#}=1_{\left[X \underline{p}^{\#}\right]+1} .
$$

Ad $(2)$. In $\mathcal{C}^{+}\left(\bar{\Delta}_{n+1}^{\#}\right)$, we have

$$
\left.\left(X \underline{\mathfrak{f}}_{n}\right) \vartheta_{n+1}=\left(X \vartheta_{2 n+1}\right) \underline{\mathfrak{f}}_{n}=\left(1_{[X]+1}\right) \underline{\mathfrak{f}}_{n}=1_{\left[X \underline{\mathfrak{f}}_{n}\right]}\right]^{+1}
$$

Ad (3). We have to show that

$$
X, Y \in \operatorname{ObC}^{+, \vartheta=1}\left(\bar{\Delta}_{n}^{\#}\right) \Longleftrightarrow X \oplus Y \in \mathrm{ObC}^{+, \vartheta=1}\left(\bar{\Delta}_{n}^{\#}\right) .
$$

But since $\vartheta_{n}$ is a morphism between additive functors, we have $(X \oplus Y) \vartheta_{n}=$ $1_{[X \oplus Y]^{+1}}$ if and only if $X \vartheta_{n}=1_{[X]^{+1}}$ and $Y \vartheta_{n}=1_{[Y]^{+1}}$. In fact, $(X \oplus Y) \vartheta_{n}$ identifies with $\left(\begin{array}{cc}X \vartheta_{n} & 0 \\ 0 & Y \vartheta_{n}\end{array}\right)$.

Ad (4). Since $\left.f\right|_{\dot{\Delta}_{n}}$ is an isomorphism in $\mathcal{C}\left(\dot{\Delta}_{n}\right)$, so is its image in $\mathcal{C}\left(\dot{\Delta}_{n}\right)$. Hence the image of $f$ in $\mathcal{C}^{+}\left(\bar{\Delta}_{n}^{\#}\right)$ is an isomorphism by Proposition 2.6. Consider the commutative quadrangle

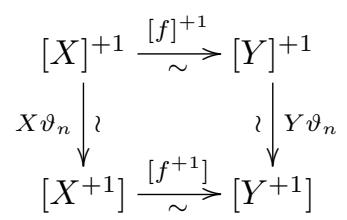

in $\underline{\mathcal{C}^{+}\left(\bar{\Delta}_{n}^{\#}\right)}$. Since $[f]^{+1}=\left[f^{+1}\right]$ by assumption, we conclude from $X \vartheta_{n}=1_{[X]^{+1}}$ 
that $Y \vartheta_{n}=1_{[Y]+1}$.

Ad (5). It suffices to show that given $0 \leqslant i \leqslant j \leqslant n$, the morphism $f_{j / i}$ is an isomorphism in $\mathcal{C}$. In fact, we have a morphism of exact sequences

$$
\left(f_{i / 0}, f_{j / 0}, f_{j / i}, f_{i / 0}^{+1}, f_{j / 0}^{+1}\right)
$$

in $\hat{\mathcal{C}}$, whose entries except possibly $f_{j / i}$ are isomorphisms; hence $f_{j / i}$ is isomorphic also.

Ad (6). This follows by Lemma 3.2 using (5).

Ad (7). We have $[X]^{+1} \simeq 0$ in $\underline{\mathcal{C}^{+}\left(\bar{\Delta}_{n}^{\#}\right)}$, from which

$$
X \vartheta_{n}=1_{[X]^{+1}} \in \underline{\underline{\mathcal{C}^{+}\left(\bar{\Delta}_{n}^{\#}\right)}}\left([X]^{+1},[X]^{+1}\right) .
$$

\subsection{Turning $n$-triangles}

Let $n \geqslant 2$.

Lemma 3.5. Suppose given an $n$-triangle $X \in \mathrm{ObC}^{+, \vartheta=1}\left(\bar{\Delta}_{n}^{\#}\right)$. We define the periodic n-pretriangle $Y \in \operatorname{Ob} \mathcal{C}^{+ \text {,periodic }}\left(\bar{\Delta}_{n}^{\#}\right)$ by letting

$$
\left(Y_{j / i} \stackrel{y}{\longrightarrow} Y_{j^{\prime} / i^{\prime}}\right):=\left(X_{i^{+1} / j} \stackrel{x}{\longrightarrow} X_{i^{\prime+1} / j^{\prime}}\right)
$$

for $0 \leqslant i \leqslant j \leqslant n$ and $0 \leqslant i^{\prime} \leqslant j^{\prime} \leqslant n$ such that $i \leqslant i^{\prime}$ and $j \leqslant j^{\prime}$, and by letting

$$
\left(Y_{n / i} \stackrel{y}{\longrightarrow} Y_{0^{+1} / i}\right):=\left(X_{i^{+1} / n} \stackrel{-x}{\longrightarrow} X_{i^{+1} / 0^{+1}}\right)
$$

for $0 \leqslant i \leqslant n$. Then $[X]_{-}^{+1}:=Y$ is an $n$-triangle.

Proof. Let

$$
\begin{aligned}
2 \bar{\Delta}_{n-1} & \stackrel{h_{n}}{\longrightarrow} \bar{\Delta}_{n} \\
i^{+j} & \longmapsto \begin{cases}(i+1)^{+j / 2} & \text { if } j \equiv_{2} 0 \\
0^{+(j+1) / 2} & \text { if } j \equiv_{2} 1,\end{cases}
\end{aligned}
$$

where $i \in[0, n-1]$ and $j \in \mathbf{Z}$. The map $h_{n}$ is a morphism of periodic posets. We claim that

$$
Y=X h_{n}^{\#} \mathfrak{f}_{n-1} .
$$

Once this claim is shown, we are done, by Lemma 3.4.(1, 2).

Note that $\left(X h_{n}^{\#}\right)_{l / k}=X_{l h_{n} / k h_{n}}$ for $k, l \in 2 \bar{\Delta}_{n-1}$ with $k \leqslant l$. For $0 \leqslant i \leqslant n$ and $1 \leqslant j \leqslant 0^{+1}$, we obtain

$$
\begin{aligned}
& \left(X h_{n}^{\#} \mathfrak{f}_{n-1}\right)_{j / i} \\
= & \begin{cases}\left(X h_{n}^{\#}\right)_{(j-1)^{+1} /(j-1)} & \text { for } i=0 \text { and } 1 \leqslant j \leqslant n \\
\left(X h_{n}^{\#}\right)_{(i-1)^{+2} /(i-1)^{+1}} & \text { for } 1 \leqslant i \leqslant n \text { and } j=0^{+1} \\
\left(X h_{n}^{\#}\right)_{(j-1)^{+1} /(i-1)^{+1}} \oplus\left(X h_{n}^{\#}\right)_{(i-1)^{+2} /(j-1)} & \text { for } 1 \leqslant i \leqslant j \leqslant n \\
0 & \text { for } i=0 \text { and } j=0^{+1}\end{cases} \\
= & \begin{cases}X_{0^{+1} / j} & \text { for } i=0 \text { and } 1 \leqslant j \leqslant n \\
X_{i+1} / 0^{+1} & \text { for } 1 \leqslant i \leqslant n \text { and } j=0^{+1} \\
X_{i+1} / j & \text { for } 1 \leqslant i \leqslant j \leqslant n \\
0 & \text { for } i=0 \text { and } j=0^{+1},\end{cases}
\end{aligned}
$$


and also the morphisms result as claimed.

\subsection{Application to the axioms of Verdier}

Recall that $(\mathcal{C}, \mathrm{T}, \vartheta)$ is a Heller triangulated category.

Proposition 3.6. Suppose that idempotents split in $\mathcal{C}$. The tuple $(\mathcal{C}, \mathrm{T})$, equipped with the set of 2-triangles as the set of distinguished triangles, is a triangulated category in the sense of Verdier [29, Def. 1-1].

Proof. We number the axioms of Verdier as in loc. cit.

Ad (TR 1). Stability under isomorphism of the set of distinguished triangles follows from Lemma 3.4.(4).

The possible extension of a morphism to a distinguished triangle follows by Lemma 3.1.

The distinguished triangle $(X, X, 0)$ on the identity of an object $X$ in $\mathcal{C}$ follows by Lemma 3.4.(7). Alternatively, one can use that each morphism is contained in a distinguished triangle and the fact that a distinguished triangle is a long exact sequence in $\hat{\mathcal{C}}$.

Ad (TR 2). Suppose given a distinguished triangle

$$
X \stackrel{u}{\longrightarrow} Y \stackrel{v}{\longrightarrow} Z \stackrel{w}{\longrightarrow} X^{+1} .
$$

By 3.5, we obtain the distinguished triangle

$$
X^{+1} \stackrel{u^{+1}}{\longrightarrow} Y^{+1} \stackrel{v^{+1}}{\longrightarrow} Z^{+1} \stackrel{-w^{+1}}{\longrightarrow} X^{+2} .
$$

By 3.4.(1), applied to the morphism $\bar{\Delta}_{2} \longleftarrow \bar{\Delta}_{2}$ that sends 0 to $2^{-1}, 1$ to 0 and 2 to 1 , we obtain the distinguished triangle

$$
Y \stackrel{v}{\longrightarrow} Z \stackrel{-w}{\longrightarrow} X^{+1} \stackrel{u^{+1}}{\longrightarrow} Y^{+1} .
$$

By 3.4.(4), we obtain the distinguished triangle

$$
Y \stackrel{v}{\longrightarrow} Z \stackrel{w}{\longrightarrow} X^{+1} \stackrel{-u^{+1}}{\longrightarrow} Y^{+1} .
$$

Ad (TR 3). The possible completion of a morphism in $\mathcal{C}\left(\dot{\Delta}_{2}\right)$ to a morphism of distinguished triangles follows from Lemma 3.2.

Ad (TR 4). The octahedral axiom, i.e. the compatibility of "forming cones" with composition of morphisms, follows from Lemma 3.1 applied to the case $n=$ 3 , for by Lemma 3.4.(6), we may arbitrarily choose completions to distinguished triangles.

Note that 3-triangles are particular octahedra, in the language of $[\mathbf{3}, 1.1 .6]$. Using 3 -triangles, we will now verify the axiom proposed in [3, 1.1.13]. 
Lemma 3.7. Suppose given a 3-triangle $T$ in $\mathrm{Ob} \mathcal{C}^{+, \vartheta=1}\left(\left(2 \bar{\Delta}_{1}\right)^{\#}\right)$, depicted as follows.

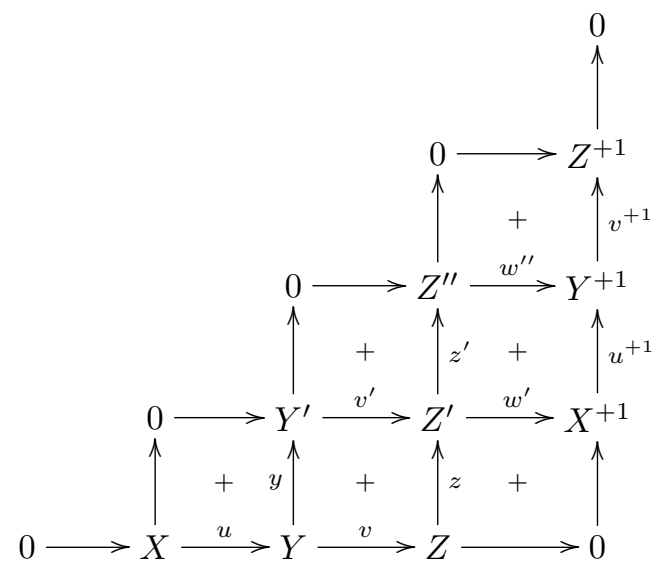

Then

$$
T \mathfrak{f}_{1}=\left(Y \stackrel{v z}{\longrightarrow} Z^{\prime} \stackrel{\left(z^{\prime} w^{\prime}\right)}{\longrightarrow} Z^{\prime \prime} \oplus X^{+1} \stackrel{\left(\begin{array}{c}
w^{\prime \prime} \\
-u^{+1}
\end{array}\right)}{\longrightarrow} Y^{+1}\right)
$$

and

$$
T s^{\#} \mathfrak{f}_{1}=\left(Z^{\prime-1} \stackrel{w^{\prime-1} u}{\longrightarrow} Y \stackrel{(y v)}{\longrightarrow} Y^{\prime} \oplus Z \stackrel{\left(\begin{array}{c}
v^{\prime} \\
-z
\end{array}\right)}{\longrightarrow} Z^{\prime}\right)
$$

are distinguished triangles, where $2 \bar{\Delta}_{1} \stackrel{s}{\leftrightarrow} 2 \bar{\Delta}_{1}$ is the morphism of periodic posets determined by $0 s=1^{-1}, 1 s=0,0^{+1} s=1$ and $1^{+1} s=0^{+1}$.

Proof. This follows by Lemma 3.4.(1, 2).

\section{5. $\quad n$-triangles and strictly exact functors}

Let $\mathcal{C} \stackrel{F}{\longrightarrow} \mathcal{C}^{\prime}$ be a strictly exact functor between Heller triangulated categories $(\mathcal{C}, \boldsymbol{T}, \vartheta)$ and $\left(\mathcal{C}^{\prime}, \mathrm{T}^{\prime}, \vartheta^{\prime}\right)$. Let $n \geqslant 0$.

Lemma 3.8. Given an $n$-triangle $X \in \mathrm{Ob} \mathcal{C}^{+, \vartheta=1}\left(\bar{\Delta}_{n}^{\#}\right)$, the diagram $X\left(F^{+}\left(\bar{\Delta}_{n}^{\#}\right)\right)$, obtained by pointwise application of $F$ to $X$, is an $n$-triangle, i.e. an object of $\mathcal{C}^{\prime+, \vartheta^{\prime}=1}\left(\bar{\Delta}_{n}^{\#}\right)$.

Proof. Using $F \mathrm{~T}^{\prime}=\mathrm{T} F$ as well as $[X]^{+1}=\left[X^{+1}\right]$, we obtain

$$
\begin{array}{rlrl}
{\left[X F^{+}\left(\bar{\Delta}_{n}^{\#}\right)\right]^{+1}} & =X\left(F^{+}\left(\bar{\Delta}_{n}^{\#}\right)\right)\left(\mathcal{C}^{+}\left(\mathrm{T}_{n}\right)\right) & =X\left(F^{+}\left(\mathrm{T}_{n}\right)\right) \\
& =X\left(\mathcal{C}^{+}\left(\mathrm{T}_{n}\right)\right)\left(F^{+}\left(\bar{\Delta}_{n}^{\#}\right)\right) & =[X]^{+1}\left(F^{+}\left(\bar{\Delta}_{n}^{\#}\right)\right) \\
& =\left[X^{+1}\right]\left(F^{+}\left(\bar{\Delta}_{n}^{\#}\right)\right) & & =X\left(\mathrm{~T}^{+}\left(\bar{\Delta}_{n}^{\#}\right)\right)\left(F^{+}\left(\bar{\Delta}_{n}^{\#}\right)\right) \\
& =X\left(\left(\mathrm{~T}^{\prime} F\right)^{+}\left(\bar{\Delta}_{n}^{\#}\right)\right) & & =X\left(\left(F^{T^{\prime}}\right)^{+}\left(\bar{\Delta}_{n}^{\#}\right)\right) \\
& =X\left(F^{+}\left(\bar{\Delta}_{n}^{\#}\right)\right)\left(\mathrm{T}^{\prime+}\left(\bar{\Delta}_{n}^{\#}\right)\right) & =\left[\left(X\left(F^{+}\left(\bar{\Delta}_{n}^{\#}\right)\right)\right)^{+1}\right] .
\end{array}
$$

Moreover,

$$
X\left(F^{+}\left(\bar{\Delta}_{n}^{\#}\right)\right) \vartheta_{n}^{\prime}=X \vartheta_{n}\left(F^{+}\left(\bar{\Delta}_{n}^{\#}\right)\right)=1_{[X]^{+1}}\left(F^{+}\left(\bar{\Delta}_{n}^{\#}\right)\right)=1_{\left[X F^{+}\left(\bar{\Delta}_{n}^{\#}\right)\right]^{+1}} .
$$




\subsection{A remark on spectral sequences}

VERDIER calls certain pretriangles objets spectraux (spectral objects); see [28, Sec. II.4]. We shall explain the connection to spectral sequences in our language.

Consider the linearly ordered set $\mathbf{Z}_{\infty}:=\{-\infty\} \sqcup \mathbf{Z} \sqcup\{+\infty\}$. Let $\overline{\mathbf{Z}}_{\infty}^{\# \#}$ be the subposet of $\overline{\mathbf{Z}}_{\infty}^{\#}\left(\Delta_{1}\right)$ consisting of those $\delta / \beta / / \gamma / \alpha$ for which

$$
\delta^{-1} \leqslant \alpha \leqslant \beta \leqslant \gamma \leqslant \delta \leqslant \alpha^{+1},
$$

where $\alpha, \beta, \gamma, \delta \in \overline{\mathbf{Z}}_{\infty}$. A spectral object, in a slightly different sense from $\left[28\right.$, II.4.1.2], is an object of $\mathcal{C}^{+}\left(\overline{\mathbf{Z}}_{\infty}^{\#}\right)$. The spectral sequence functor

$$
\begin{aligned}
\mathcal{C}^{+}\left(\overline{\mathbf{Z}}_{\infty}^{\#}\right) \stackrel{\mathrm{E}}{\longrightarrow} \hat{\mathcal{C}}\left(\overline{\mathbf{Z}}_{\infty}^{\# \#}\right) & \\
X & \longmapsto X \mathrm{E},
\end{aligned}
$$

is defined by

$$
X \mathrm{E}(\delta / \beta / / \gamma / \alpha):=\operatorname{Im}\left(X_{\gamma / \alpha} \longrightarrow X_{\delta / \beta}\right)
$$

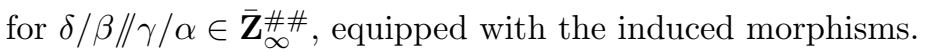

Lemma 3.9. Given $\alpha, \beta, \gamma, \delta, \varepsilon \in \overline{\mathbf{Z}}_{\infty}$ such that

$$
\varepsilon^{-1} \leqslant \alpha \leqslant \beta \leqslant \gamma \leqslant \delta \leqslant \varepsilon \leqslant \alpha^{+1},
$$

and given $X \in \mathrm{ObC}^{+}\left(\overline{\mathbf{Z}}_{\infty}^{\#}\right)$, the morphisms appearing in $X \mathrm{E}$ form a short exact sequence

$$
X \mathrm{E}(\varepsilon / \beta / / \gamma / \alpha) \rightarrow X \mathrm{E}(\varepsilon / \beta / / \delta / \alpha) \rightarrow X \mathrm{E}(\varepsilon / \gamma / / \delta / \alpha) .
$$

Proof. This follows by Lemma A.22, applied to the diagram

$$
(X_{\gamma / \alpha}, X_{\delta / \alpha}, X_{\varepsilon / \alpha}, X_{\gamma / \beta}, X_{\delta / \beta}, X_{\varepsilon / \beta}, \underbrace{X_{\gamma / \gamma}}_{=0}, X_{\delta / \gamma}, X_{\varepsilon / \gamma}) .
$$

Note that we may apply a shift $\beta / \alpha \longmapsto \alpha^{+1} / \beta$ to the indices, i.e. an outer shift to $X$, before applying Lemma 3.9 to get another short exact sequence.

The usual exact sequences of spectral sequence terms can be derived from Lemma 3.9. Cf. [28, II.4.2.6], [5, App.].

\section{The stable category of a Frobenius category is Heller tri- angulated}

Let $\mathcal{F}=(\mathcal{F}, T, \mathrm{I}, \iota, \mathrm{P}, \pi)$ be a functorial Frobenius category; see Definition A.5.(3). Let $\mathcal{B} \subseteq \mathcal{F}$ denote the full subcategory of objects in the image of $\mathrm{I}$, coinciding with the full subcategory of the objects in the image of $\mathrm{P}$; then $\mathcal{B}$ is a sufficiently large full subcategory of bijectives in $\mathcal{F}$.

We shall prove in Theorem 4.6 below that the classical stable category $\underline{\mathcal{F}}$ carries a Heller triangulation.

\subsection{Definition of $\mathcal{F}^{\square}\left(\bar{\Delta}_{n}^{\#}\right)$, modelling $\underline{\mathcal{F}}^{+}\left(\bar{\Delta}_{n}^{\#}\right)$}

We shall model, in the sense of Proposition 4.5 below, the category $\underline{\mathcal{F}}^{+}\left(\bar{\Delta}_{n}^{\#}\right)$ by a category $\mathcal{F}^{\square}\left(\bar{\Delta}_{n}^{\#}\right)$. Morally, we represent weak squares $(+)$ in $\underline{\mathcal{F}}$ by pure squares 
$(\square)$ in $\mathcal{F}$. To do so, we have to represent the zeroes on the boundaries by bijective objects.

Let $n \geqslant 0$. Concerning the notion of a pure square, see $\S$ A.4. Let $\mathcal{F}^{\square}\left(\bar{\Delta}_{n}^{\#}\right) \subseteq$ $\mathcal{F}\left(\bar{\Delta}_{n}^{\#}\right)$ be the full subcategory defined by

$$
\operatorname{Ob} \mathcal{F}^{\square}\left(\bar{\Delta}_{n}^{\#}\right):=
$$

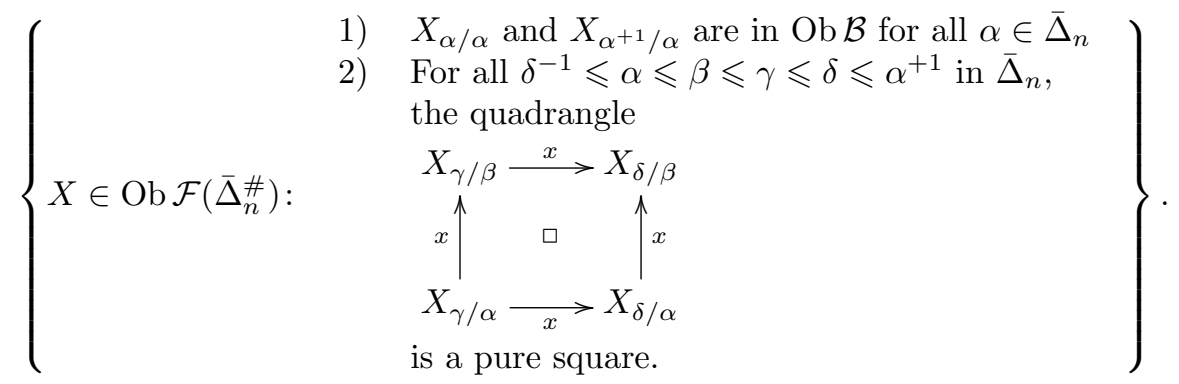

Given $n, m \geqslant 0$, a morphism $\bar{\Delta}_{n} \stackrel{p}{\longleftarrow} \bar{\Delta}_{m}$ induces a morphism $\mathcal{F}^{\square}\left(p^{\#}\right)$, which is usually, and by abuse of notation, denoted by $p^{\#}$.

Given an exact functor $\mathcal{F} \stackrel{F}{\longrightarrow} \tilde{\mathcal{F}}$ between functorial Frobenius categories that sends bijectives to bijectives, we obtain an induced functor

$$
\mathcal{F}^{\square}\left(\bar{\Delta}_{n}^{\#}\right) \stackrel{F^{\square}\left(\bar{\Delta}_{n}^{\#}\right)}{\longrightarrow} \tilde{\mathcal{F}}^{\square}\left(\bar{\Delta}_{n}^{\#}\right)
$$

by pointwise application of $F$.

Denote by

$$
\begin{aligned}
\mathcal{F}^{\square}\left(\bar{\Delta}_{n}^{\#}\right) \stackrel{M}{\longrightarrow} \underline{\underline{\mathcal{F}}}^{+}\left(\bar{\Delta}_{n}^{\#}\right) \\
\mathcal{F}^{\square}\left(\bar{\Delta}_{n}^{\#}\right) \stackrel{M^{\prime}}{\longrightarrow} \underline{\mathcal{F}}^{+}\left(\bar{\Delta}_{n}^{\#}\right) \\
\underline{\mathcal{F}}^{+}\left(\bar{\Delta}_{n}^{\#}\right) \stackrel{M^{\prime \prime}}{\longrightarrow} \underline{\underline{\mathcal{F}}}^{+}\left(\bar{\Delta}_{n}^{\#}\right) \\
\mathcal{F} \stackrel{\text { ․․․․ }}{\underline{\mathcal{F}}} \\
\underline{\mathcal{F}}\left(\dot{\Delta}_{n}\right) \stackrel{N^{\prime}}{\longrightarrow} \underline{\underline{\mathcal{F}}\left(\dot{\Delta}_{n}\right)}
\end{aligned}
$$

the respective residue class functors, well defined by Lemma A.31. In particular, $M=M^{\prime} M^{\prime \prime}$.

\section{2. $\quad$ Folding for $\mathcal{F}^{\square}\left(\bar{\Delta}_{n}^{\#}\right)$}

We model, in the sense of Remark 4.1, the folding operation $f$ introduced in $\S 1.2 .2$.

Suppose given $n \geqslant 0$. Let the periodic functor

$$
\begin{aligned}
\mathcal{F}^{\square}\left(\left(2 \bar{\Delta}_{n}\right)^{\#}\right) & \stackrel{\tilde{\mathfrak{f}}_{n}}{\longrightarrow} \mathcal{F}^{\square}\left({\overline{\rho \sqcup \Delta_{n}}}^{\#}\right) \\
X & \longmapsto X \tilde{\mathfrak{f}}_{n}
\end{aligned}
$$


Homology, Homotopy and Applications, vol. 9(2), 2007

be determined by the following data. Writing $Y:=X \tilde{\mathfrak{f}}_{n}$, we let

$$
\begin{aligned}
& \left(Y_{\alpha / \rho} \stackrel{y}{\longrightarrow} Y_{\beta / \rho}\right):=\left(X_{\alpha+1 / \alpha} \stackrel{x}{\longrightarrow} X_{\beta+1 / \beta}\right) \\
& Y_{\rho / \rho}:=0 \\
& Y_{\rho^{+1} / \rho}:=0 \\
& \left(Y_{\beta / \rho} \stackrel{y}{\longrightarrow} Y_{\beta / \alpha}\right):=\left(X_{\beta^{+1} / \beta} \stackrel{(x x)}{\longrightarrow} X_{\beta^{+1} / \alpha^{+1}} \oplus X_{\alpha^{+2} / \beta}\right) \\
& \left(Y_{\beta / \alpha} \stackrel{y}{\longrightarrow} Y_{\delta / \gamma}\right):=\left(X_{\beta^{+1} / \alpha^{+1}} \oplus X_{\alpha+2 / \beta} \stackrel{\left(\begin{array}{ll}
x & 0 \\
0 & x
\end{array}\right)}{\longrightarrow} X_{\delta^{+1} / \gamma^{+1}} \oplus X_{\gamma^{+2} / \delta}\right) \\
& \left(Y_{\delta / \gamma} \stackrel{y}{\longrightarrow} Y_{\rho^{+1} / \gamma}\right):=\left(X_{\delta^{+1} / \gamma^{+1}} \oplus X_{\gamma^{+2} / \delta} \stackrel{\left(\begin{array}{c}
x \\
-x
\end{array}\right)}{\longrightarrow} X_{\gamma^{+2} / \gamma^{+1}}\right)
\end{aligned}
$$

for $\alpha, \beta, \gamma, \delta \in \Delta_{n}$ with $\alpha \leqslant \beta$, with $\gamma \leqslant \delta$ and with $\beta / \alpha \leqslant \delta / \gamma$. The remaining morphisms are given by composition.

We claim that $X \tilde{\mathfrak{f}}_{n}$ is an object of $\mathcal{F}^{\square}\left({\overline{\rho \sqcup \Delta_{n}}}^{\#}\right)$.

In fact, by Lemma A.12, we are reduced to considering the quadrangles of $Y$ inside $\Delta_{n}^{\Delta \nabla}$, i.e. the quadrangles

(i) on $(\gamma / \alpha, \delta / \alpha, \gamma / \beta, \delta / \beta)$ for $\alpha, \beta, \gamma, \delta \in \Delta_{n}$ with $\alpha \leqslant \beta \leqslant \gamma \leqslant \delta$;

(ii) on $(\gamma / \rho, \delta / \rho, \gamma / \beta, \delta / \beta)$ for $\beta, \gamma, \delta \in \Delta_{n}$ with $\beta \leqslant \gamma \leqslant \delta$;

(iii) on $\left(\gamma / \alpha, \rho^{+1} / \alpha, \gamma / \beta, \rho^{+1} / \beta\right)$ for $\alpha, \beta, \gamma \in \Delta_{n}$ with $\alpha \leqslant \beta \leqslant \gamma$;

(iv) and on $\left(\beta / \rho, \rho^{+1} / \rho, \beta / \alpha, \rho^{+1} / \alpha\right)$ for $\alpha, \beta \in \Delta_{n}$ with $\alpha \leqslant \beta$.

Another application of loc. cit. reduces case (i) to case (ii) (or (iii)). Still another application of loc. cit. reduces the cases (ii) and (iii) to case (iv). Now the quadrangle in case (iv) is in fact a pure square, as follows from $X \in \mathrm{ObF}^{\square}\left(\bar{\Delta}_{n}^{\#}\right)$ and the definition of a pure square via its pure short exact diagonal sequence.

The construction of $Y$ is functorial in $X$.

Remark 4.1. We have $\tilde{\mathfrak{f}}_{n} M^{\prime}=M^{\prime} \mathfrak{f}_{n}$, and thus $\tilde{\mathfrak{f}}_{n} M=M \mathfrak{f}_{n}$ for $n \geqslant 0$.

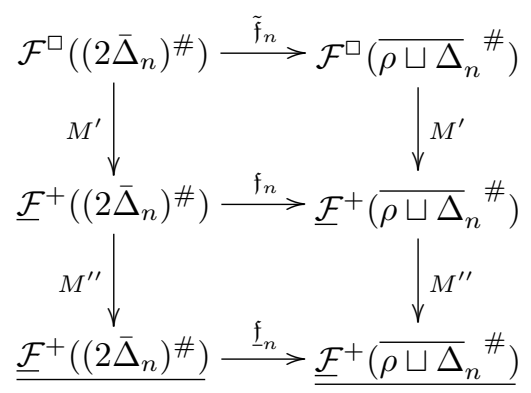

Example 4.2. Let $n=2$. Note that $2 \bar{\Delta}_{2} \simeq \bar{\Delta}_{5}$. Let $X \in \mathrm{Ob} \mathcal{F}^{\square}\left(\left(2 \bar{\Delta}_{2}\right)^{\#}\right)$, depicted as follows. 


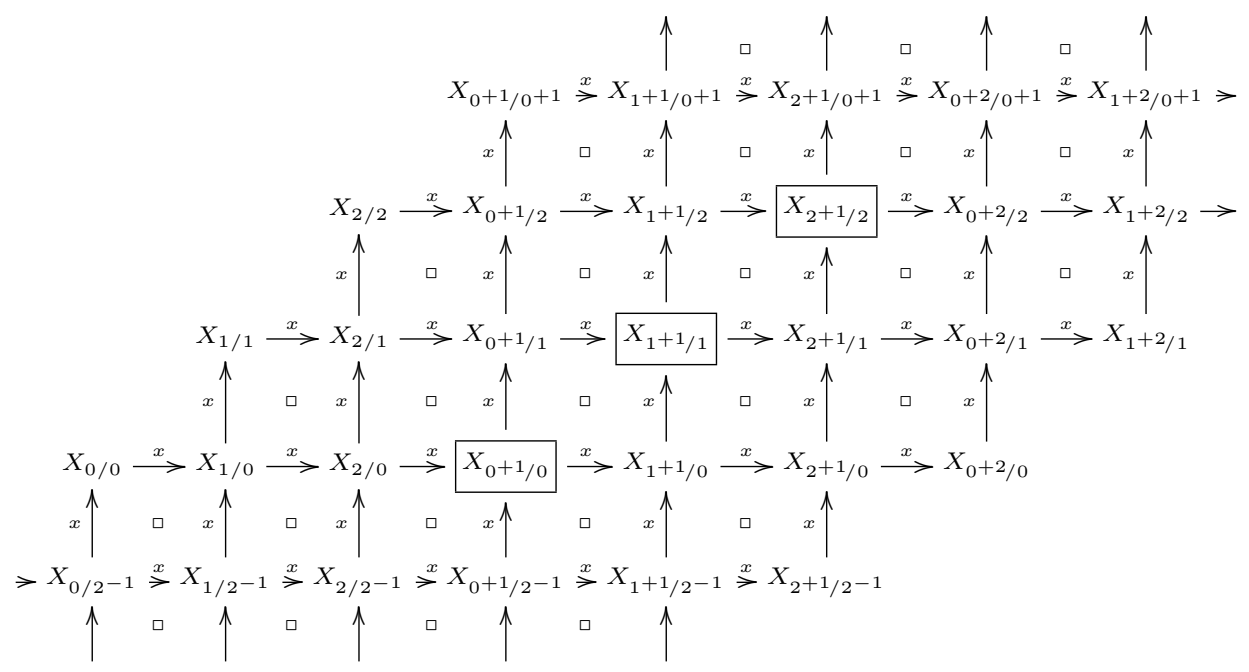

Note that the objects on the boundary of the diagram,

$$
\begin{gathered}
\ldots, X_{2^{-1} / 2^{-1}}, X_{0 / 0}, X_{1 / 1}, X_{2 / 2}, X_{0^{+1} / 0^{+1}}, \ldots \\
\ldots, X_{2^{+1} / 2^{-1}}, X_{0^{+2} / 0}, X_{1+2} / 1, X_{2^{+2} / 2}, X_{0^{+3} / 0^{+1}}, \ldots
\end{gathered}
$$

are all supposed to be in $\mathrm{Ob} \mathcal{B}$.

Note that $\rho \sqcup \Delta_{2} \simeq \Delta_{3}$. Folding turns $X$ into $X \tilde{\mathfrak{f}}_{2} \in \operatorname{Ob} \mathcal{F}^{\square}\left({\overline{\rho \sqcup \Delta_{2}}}^{\#}\right)$, depicted as follows.

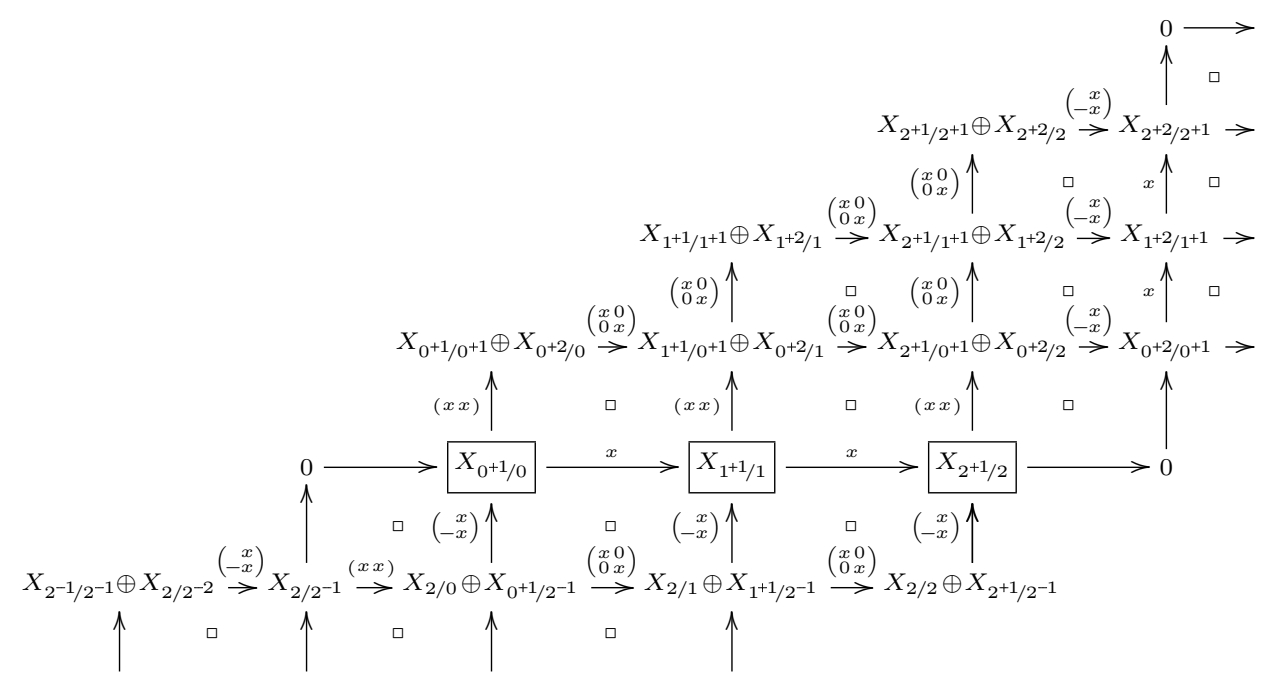

\subsection{Some 1-epimorphic functors}

Let $n \geqslant 0$. 
Lemma 4.3. The restriction functor

$$
\begin{aligned}
\mathcal{F}^{\square}\left(\bar{\Delta}_{n}^{\#}\right) & \stackrel{\left.(-)\right|_{\dot{\Delta}_{n}}}{\longrightarrow} \mathcal{F}\left(\dot{\Delta}_{n}\right) \\
X & \left.\longmapsto X\right|_{\dot{\Delta}_{n}}
\end{aligned}
$$

is 1-epimorphic.

Proof. We claim that the functor $\left.(-)\right|_{\dot{\Delta}_{n}}$ satisfies the requirements (i, ii) of Corollary A.36, which then implies that it is 1-epimorphic.

Suppose given $Y \in \operatorname{Ob} \mathcal{F}\left(\dot{\Delta}_{n}\right)$. We construct an object $\tilde{Y}$ of $\mathcal{F}^{\square}\left(\bar{\Delta}_{n}^{\#}\right)$ such that $\left.\tilde{Y}\right|_{\dot{\Delta}_{n}}=Y$ by the following procedure.

Write $\bar{\Delta}_{n}^{\Delta, \cdot}:=\bar{\Delta}_{n}^{\Delta} \backslash\{0 / 0\}$ and $\bar{\Delta}_{n}^{\nabla, \cdot}:=\bar{\Delta}_{n}^{\nabla} \backslash\left\{0^{+1} / 0\right\} ;$ cf. $\S 2.1 .1$.

On $\bar{\Delta}_{n}^{\Delta, \cdot}$, we proceed by induction to construct a diagram for which, moreover, the morphisms $\tilde{Y}_{\gamma / \alpha} \longrightarrow \tilde{Y}_{\gamma / \beta}$ are purely monomorphic for all $\alpha, \beta, \gamma \in \bar{\Delta}_{n}$ with $0 \leqslant \alpha \leqslant \beta \leqslant \gamma \leqslant \alpha^{+1}$, and, moreover, for which $\tilde{Y}_{\alpha^{+1} / \alpha}=0$ for all $0 \leqslant \alpha$.

First of all, let $\left.\tilde{Y}\right|_{\dot{\Delta}_{n}}:=Y$.

Assume given $l \geqslant 0$ such that $\tilde{Y}_{\kappa^{\Delta}\left(l^{\prime}\right)}$, together with all diagram morphisms pointing to position $\kappa^{\Delta}\left(l^{\prime}\right)$, is already constructed for all $l^{\prime}<l$, but such that $\tilde{Y}_{\kappa^{\Delta}(l)}$ is not yet constructed; cf. $\S 2.1 .2$.

If $\kappa^{\Delta}(l)$ is of the form $\alpha / \alpha$ for some $\alpha \in \bar{\Delta}_{n}$ with $0<\alpha$, then choose a pure monomorphism $\tilde{Y}_{\alpha /(\alpha-1)} \longrightarrow \tilde{Y}_{\alpha / \alpha}$ into an object $\tilde{Y}_{\alpha / \alpha}$ of $\mathcal{B}$. The chosen morphism is not necessarily equal to $\tilde{Y}_{\alpha /(\alpha-1)} \iota$.

If $\kappa^{\Delta}(l)=\alpha^{+1} / \alpha$ for some $\alpha \in \bar{\Delta}_{n}$ with $0 \leqslant \alpha$, then let $\tilde{Y}_{\alpha^{+1} / \alpha}:=0$.

If $\kappa^{\Delta}(l)$ is of the form $\beta / \alpha$ for some $\alpha, \beta \in \bar{\Delta}_{n}$ with $0<\alpha<\beta<\alpha^{+1}$, then we let

$$
\left(\tilde{Y}_{(\beta-1) /(\alpha-1)}, \tilde{Y}_{(\beta-1) / \alpha}, \tilde{Y}_{\beta /(\alpha-1)}, \tilde{Y}_{\beta / \alpha}\right)
$$

be a pushout. Recall that by induction assumption, $\tilde{Y}_{(\beta-1) /(\alpha-1)} \rightarrow \tilde{Y}_{(\beta-1) / \alpha}$ is purely monomorphic. So $\tilde{Y}_{\beta /(\alpha-1)} \longrightarrow \tilde{Y}_{\beta / \alpha}$ is purely monomorphic as well.

On $\bar{\Delta}_{n}^{\nabla, \cdot}$, we proceed dually, and finally glue along $\dot{\Delta}_{n}$ to obtain the sought $\tilde{Y}$.

Ad (i). The restriction map $\mathcal{F}\left(\bar{\Delta}_{n}^{\#}\right)\left(\tilde{Y}_{1}, \tilde{Y}_{2}\right) \stackrel{\left.(-)\right|_{\Delta_{n}}}{\longrightarrow} \mathcal{F}\left(\dot{\Delta}_{n}\right)\left(Y_{1}, Y_{2}\right)$ is surjective for $Y_{1}, Y_{2} \in \mathrm{Ob} \mathcal{F}\left(\dot{\Delta}_{n}\right)$, as we see by induction, using bijectivity to prolong morphisms and universal properties of occurring pushouts and pullbacks.

Ad (ii). Suppose given $X \in \operatorname{Ob} \mathcal{F}^{\square}\left(\bar{\Delta}_{n}^{\#}\right)$. Let $X^{\prime \prime}:=\left(\left.X\right|_{\dot{\Delta}_{n}}\right)^{\sim} \in \operatorname{Ob} \mathcal{F}^{\square}\left(\bar{\Delta}_{n}^{\#}\right)$. Let $X^{\prime} \in \operatorname{Ob} \mathcal{F}^{\square}\left(\bar{\Delta}_{n}^{\#}\right)$ be defined by $\left.\left.X^{\prime}\right|_{\bar{\Delta}_{n}^{\Delta,} \cdot=X^{\prime \prime}}\right|_{\bar{\Delta}_{n}^{\Delta,},}$ and by $\left.X^{\prime}\right|_{\bar{\Delta}_{n}^{\nabla}, \cdot}=\left.X\right|_{\bar{\Delta}_{n}^{\nabla}, \cdot}$

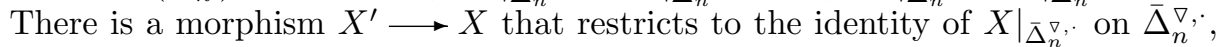
and hence to the identity of $\left.X\right|_{\dot{\Delta}_{n}}$ on $\dot{\Delta}_{n}$.

There is a morphism $X^{\prime} \longrightarrow X^{\prime \prime}$ that restricts to the identity of $\left.X^{\prime \prime}\right|_{\bar{\Delta}_{n}^{\Delta,}}$ on $\bar{\Delta}_{n}^{\Delta, \cdot}$, and hence to the identity of $\left.X\right|_{\dot{\Delta}_{n}}$ on $\dot{\Delta}_{n}$.

Now suppose given $X_{1}, X_{2} \in \mathrm{Ob} \mathcal{F}^{\square}\left(\bar{\Delta}_{n}^{\#}\right)$ such that $\left.X_{1}\right|_{\dot{\Delta}_{n}}=\left.X_{2}\right|_{\dot{\Delta}_{n}}$. Then there is a sequence of morphisms

$$
X_{1} \longleftarrow X_{1}^{\prime} \longrightarrow X_{1}^{\prime \prime}=X_{2}^{\prime \prime} \longleftarrow X_{2}^{\prime} \longrightarrow X_{2}
$$

each of which restricts to the identity of $\left.X_{1}\right|_{\dot{\Delta}_{n}}=\left.X_{2}\right|_{\dot{\Delta}_{n}}$ on $\dot{\Delta}_{n}$, as required. 
Lemma 4.4. The functors

$$
\begin{aligned}
& \mathcal{F}\left(\dot{\Delta}_{n}\right) \stackrel{N\left(\dot{\Delta}_{n}\right)}{\longrightarrow} \stackrel{\mathcal{F}\left(\dot{\Delta}_{n}\right)}{\mathcal{F}\left(\dot{\Delta}_{n}\right) \stackrel{N\left(\dot{\Delta}_{n}\right) N^{\prime}}{\longrightarrow}} \underline{\underline{\mathcal{F}}\left(\dot{\Delta}_{n}\right)}
\end{aligned}
$$

are 1-epimorphic.

Proof. Since $N^{\prime}$ is full and dense, it is 1-epimorphic by Corollary A.37. Therefore, it suffices to show that $N\left(\dot{\Delta}_{n}\right)$ is 1-epimorphic.

We will apply Lemma A.35. Choosing representatives of the morphisms $Z_{i} \longrightarrow$ $Z_{i+1}$ occurring in an object $Z$ of $\mathcal{F}\left(\dot{\Delta}_{n}\right)$, where $i \in[1, n-1]$, we see that $N\left(\dot{\Delta}_{n}\right)$ is dense.

To fulfill condition (C) of loc. cit., we will show that given $X, Y \in \mathrm{Ob} \mathcal{F}\left(\dot{\Delta}_{n}\right)$ and a morphism $(X)\left(N\left(\dot{\Delta}_{n}\right)\right) \stackrel{f}{\longrightarrow}(Y)\left(N\left(\dot{\Delta}_{n}\right)\right)$, there are morphisms $X^{\prime} \stackrel{h}{\longrightarrow} X$ and $X^{\prime} \stackrel{f^{\prime}}{\longrightarrow} Y$ in $\mathcal{F}\left(\dot{\Delta}_{n}\right)$ such that $(h)\left(N\left(\dot{\Delta}_{n}\right)\right)$ is an isomorphism and such that $(h)\left(N\left(\dot{\Delta}_{n}\right)\right) f=\left(f^{\prime}\right)\left(N\left(\dot{\Delta}_{n}\right)\right)$.

We proceed by induction on $k \in[1, n]$. Suppose given a diagram

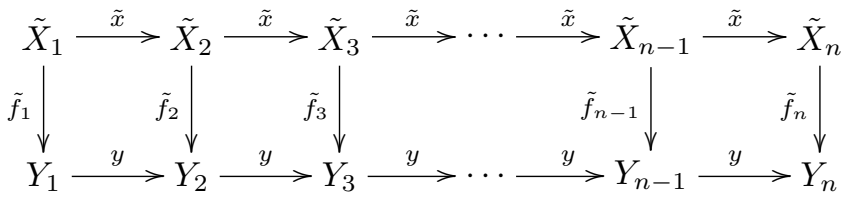

in $\mathcal{F}$ such that $\tilde{x} \tilde{f}_{i+1}=\tilde{f}_{i} y$ for $i \in[1, k-1]$, and such that $\tilde{x} \tilde{f}_{i+1} \equiv_{\mathcal{B}} \tilde{f}_{i} y$ for $i \in$ $[k, n]$, and a morphism $\tilde{X} \stackrel{\tilde{h}}{\longrightarrow} X$ in $\mathcal{F}\left(\dot{\Delta}_{n}\right)$ such that $(\tilde{h})\left(N\left(\dot{\Delta}_{n}\right)\right)$ is an isomorphism and such that $(\tilde{h})\left(N\left(\dot{\Delta}_{n}\right)\right) f$ is the morphism in $\mathcal{F}\left(\dot{\Delta}_{n}\right)$ represented by $\tilde{f}$.

If $k<n$, we shall construct a morphism $\tilde{X}^{\prime} \stackrel{\tilde{h}^{\prime}}{\longrightarrow} \tilde{X}$ in $\mathcal{F}\left(\dot{\Delta}_{n}\right)$ with each $\tilde{h}_{i}^{\prime} N$ being an isomorphism, and a diagram

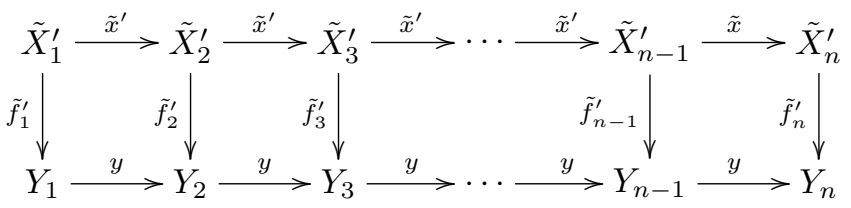

in $\mathcal{F}$ such that $\tilde{x}^{\prime} \tilde{f}_{i+1}^{\prime}=\tilde{f}_{i}^{\prime} y$ for $i \in[1, k]$, such that moreover $\tilde{x}^{\prime} \tilde{f}_{i+1}^{\prime}-\tilde{f}_{i}^{\prime} y \equiv_{\mathcal{B}} 0$ for $i \in[k+1, n]$, and such that $\tilde{h}_{i}^{\prime} \tilde{f}_{i}-\tilde{f}_{i}^{\prime} \equiv_{\mathcal{B}} 0$ for all $i \in[1, n]$. For then we obtain a commutative diagram in $\underline{\mathcal{F}}\left(\dot{\Delta}_{n}\right)$

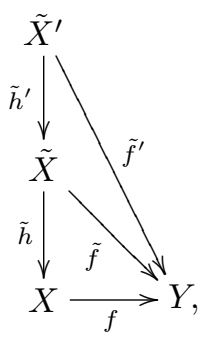


Homology, Homotopy and Applications, vol. 9(2), 2007

in which morphisms are denoted by their representatives.

Let $\tilde{X}_{k} \stackrel{j}{\rightarrow} B$ be a pure monomorphism to an object $B$ in $\mathcal{B}$, and let $\tilde{x} \tilde{f}_{k+1}-\tilde{f}_{k} y=j g$. Let

$$
\tilde{X}^{\prime}:=\left(\tilde{X}_{1} \stackrel{\tilde{x}}{\longrightarrow} \cdots \stackrel{\tilde{x}}{\longrightarrow} \tilde{X}_{k} \stackrel{(\tilde{x} j)}{\longrightarrow} \tilde{X}_{k+1} \oplus B \stackrel{\left(\begin{array}{c}
\tilde{x} \\
0
\end{array}\right)}{\longrightarrow} \tilde{X}_{k+2} \stackrel{\tilde{x}}{\longrightarrow} \cdots \stackrel{\tilde{x}}{\longrightarrow} \tilde{X}_{n}\right),
$$

and let

$$
\begin{aligned}
& \tilde{f}_{i}^{\prime}:= \begin{cases}\tilde{f}_{i} & \text { for } i \in[1, n] \backslash\{k+1\} \\
\left(\begin{array}{l}
\left.\tilde{f}_{k+1}\right) \\
-g
\end{array}\right) & \text { for } i=k+1,\end{cases} \\
& \tilde{h}_{i}^{\prime}:=\left\{\begin{array}{cc}
1_{\tilde{X}_{i}} & \text { for } i \in[1, n] \backslash\{k+1\} \\
\left(\begin{array}{cc}
1_{\tilde{x}_{k+1}} \\
0
\end{array}\right) & \text { for } i=k+1 .
\end{array}\right.
\end{aligned}
$$

Proposition 4.5. The residue class functor $\mathcal{F}^{\square}\left(\bar{\Delta}_{n}^{\#}\right) \stackrel{M}{\longrightarrow} \underline{\underline{\mathcal{F}}}^{+}\left(\bar{\Delta}_{n}^{\#}\right)$ is 1-epimorphic.

Proof. Consider the commutative quadrangle

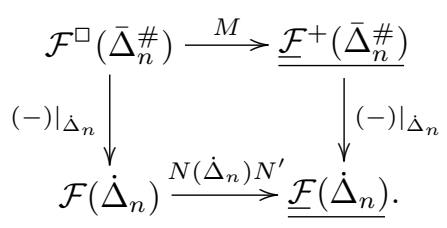

Therein, the functor $\mathcal{F}^{\square}\left(\bar{\Delta}_{n}^{\#}\right) \stackrel{\left.(-)\right|_{\Delta_{n}}}{\longrightarrow} \mathcal{F}\left(\dot{\Delta}_{n}\right)$ is 1-epimorphic by Lemma 4.3. The functor $\mathcal{F}\left(\dot{\Delta}_{n}\right) \stackrel{N\left(\dot{\Delta}_{n}\right) N^{\prime}}{\longrightarrow} \underline{\underline{\mathcal{F}}\left(\dot{\Delta}_{n}\right)}$ is 1-epimorphic by Lemma 4.4. The functor $\underline{\mathcal{F}^{+}\left(\bar{\Delta}_{n}^{\#}\right)} \stackrel{\left.(-)\right|_{\dot{\Delta}_{n}}}{\longrightarrow} \underline{\underline{F}}\left(\dot{\Delta}_{n}\right)$ is an equivalence by Proposition 2.6. Hence by Remark A.34, the functor $\mathcal{F}^{\square}\left(\bar{\Delta}_{n}^{\#}\right) \stackrel{M}{\longrightarrow} \underline{\underline{F}}^{+}\left(\bar{\Delta}_{n}^{\#}\right)$ is 1-epimorphic.

Note that we do not claim that the residue class functor $\mathcal{F}^{\square}\left(\bar{\Delta}_{n}^{\#}\right) \longrightarrow \underline{\mathcal{F}}^{+}\left(\bar{\Delta}_{n}^{\#}\right)$ is 1-epimorphic.

\subsection{Construction of $\vartheta$}

Let $n \geqslant 0$. In the notation of Lemma A.32, we let $C:=\bar{\Delta}_{n}^{\#}$, the role of the category called $\mathcal{E}$ there is played by $\mathcal{F}$ here, we let $\mathcal{G}:=\mathcal{F}^{\square}\left(\bar{\Delta}_{n}^{\#}\right)$, and finally, we let $\mathcal{H}:=\underline{\mathcal{F}}^{+}\left(\bar{\Delta}_{n}^{\#}\right)$. Note that $\underline{\mathcal{F}}^{+}\left(\bar{\Delta}_{n}^{\#}\right)$ is a characteristic subcategory of $\underline{\mathcal{F}}\left(\bar{\Delta}_{n}^{\#}\right)$. 
The tuples

$$
\begin{aligned}
I(n) & =\left(\left(I_{X, \beta / \alpha}\right)_{\beta / \alpha \in \bar{\Delta}_{n}^{\#}}\right)_{X \in \mathrm{Ob} \mathcal{F}^{\square}\left(\bar{\Delta}_{n}^{\#}\right)}\left(\begin{array}{c}
x \\
-x
\end{array}\right) \\
& :=\left(\left(X_{\beta / \alpha} \stackrel{(x x)}{\longrightarrow} X_{\beta / \beta} \oplus X_{\alpha+1} \stackrel{\longrightarrow}{\longrightarrow} X_{\alpha}^{+1 / \beta}\right)_{\beta / \alpha \in \bar{\Delta}_{n}^{\#}}\right)_{X \in \mathrm{Ob} \mathcal{F}^{\square}\left(\bar{\Delta}_{n}^{\#}\right)} \\
J(n) & =\left(\left(J_{X, \beta / \alpha}\right)_{\beta / \alpha \in \bar{\Delta}_{n}^{\#}}\right)_{X \in \mathrm{Ob} \mathcal{F}^{\square}\left(\bar{\Delta}_{n}^{\#}\right)} \\
& :=\left(\left(X_{\beta / \alpha} \stackrel{X_{\beta / \alpha} \iota}{\longrightarrow} X_{\beta / \alpha} \mathrm{I}=X_{\beta / \alpha}^{+1} \stackrel{X_{\beta / \alpha}^{+1}}{\longrightarrow} X_{\beta / \alpha}^{+1}\right)_{\beta / \alpha \in \bar{\Delta}_{n}^{\#}}\right)_{X \in \mathrm{Ob} \mathcal{F}^{\square}\left(\bar{\Delta}_{n}^{\#}\right)}
\end{aligned}
$$

are $\bar{\Delta}_{n}^{\#}$-resolving systems, inducing an isomorphism $\mathrm{T}_{I(n)} \stackrel{\alpha_{I(n), J(n)}}{\sim} \mathrm{T}_{J(n)}$ by Lemma A.32.(2). Recall that $\underline{\mathcal{F}}^{+}\left(\bar{\Delta}_{n}^{\#}\right) \stackrel{M^{\prime \prime}}{\longrightarrow} \underline{\underline{F}}^{+}\left(\bar{\Delta}_{n}^{\#}\right)$ denotes the residue class functor. We have

$$
\begin{aligned}
\mathrm{T}_{I(n)} M^{\prime \prime} & =M \underline{\underline{\mathcal{F}^{+}\left(\mathrm{T}_{n}\right)}}=M[-]^{+1} \\
\mathrm{~T}_{J(n)} M^{\prime \prime} & =M \underline{\underline{\mathrm{T}^{+}\left(\bar{\Delta}_{n}\right)}}=M\left[-^{+1}\right] .
\end{aligned}
$$

Since $M$ is 1-epimorphic by Proposition 4.5, we obtain

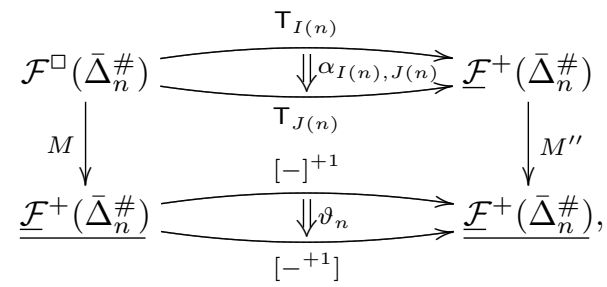

where $\vartheta_{n}$ is characterised by this commutative diagram, i.e. by

$$
\alpha_{I(n), J(n)} M^{\prime \prime}=M \vartheta_{n} .
$$

Since $\alpha_{I(n), J(n)}$ is an isomorphism, so is $\vartheta_{n}$. Varying $n$, this defines $\vartheta=\left(\vartheta_{n}\right)_{n \geqslant 0}$.

Theorem 4.6. The tuple $\vartheta=\left(\vartheta_{n}\right)_{n \geqslant 0}$ is a Heller triangulation on $\underline{\mathcal{F}}$.

Proof. According to Definition 1.5, we have to show that the following conditions $(*)$ and $(* *)$ hold.

(*) For $m, n \geqslant 0$, for a morphism $\bar{\Delta}_{n}^{p} \bar{\Delta}_{m}$ and for an object $Y \in \operatorname{Ob} \underline{\underline{\mathcal{F}^{+}}\left(\bar{\Delta}_{n}^{\#}\right)}$, we have

$$
\left(Y \underline{p}^{\#}\right) \vartheta_{m}=\left(Y \vartheta_{n}\right) \underline{p}^{\#} .
$$

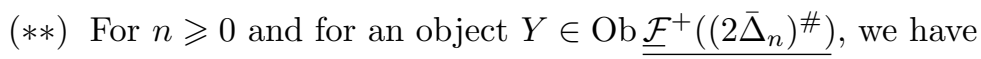

$$
\left(Y \underline{\mathfrak{f}}_{n}\right) \vartheta_{n+1}=\left(Y \vartheta_{2 n+1}\right) \underline{\mathfrak{f}}_{n} .
$$


Ad (*). Recall that $\underline{p}^{\#}$ stands for $\underline{\mathcal{F}}^{+}\left(p^{\#}\right)$, and that $p^{\#}$ stands for $\mathcal{F}^{\square}\left(p^{\#}\right)$. By

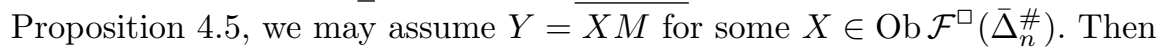

$$
\begin{aligned}
& \left(X M \underline{p}^{\#}\right) \vartheta_{m}=\left(X p^{\#} M\right) \vartheta_{m}=\left(X p^{\#} \alpha_{I(m), J(m)}\right) M^{\prime \prime} \\
& \left(X M \vartheta_{n}\right) \underline{p}^{\#}=\left(X \alpha_{I(n), J(n)} M^{\prime \prime}\right) \underline{p}^{\#}=\left(X \alpha_{I(n), J(n)} p^{\#}\right) M^{\prime \prime},
\end{aligned}
$$

so that it suffices to show that $X p^{\#} \alpha_{I(m), J(m)}=X \alpha_{I(n), J(n)} p^{\#}$.

Starting with $X p^{\#}$, the object $X p^{\#} \mathrm{~T}_{I(m)}$ is calculated by means of

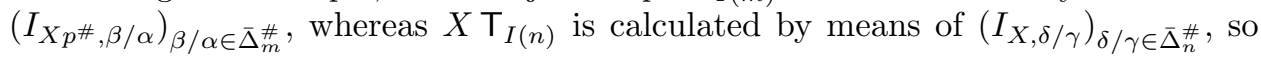
$X \mathrm{~T}_{I(n)} p^{\#}$ can be regarded as being calculated by means of $\left(I_{X, \beta p / \alpha p}\right)_{\beta / \alpha \in \bar{\Delta}_{m}^{\#}}$. But

$$
I_{X, \beta p / \alpha p}=\left(X_{\beta p / \alpha p} \stackrel{(x x)}{\longrightarrow} X_{\beta p / \beta p} \oplus X_{(\alpha p)^{+1} / \alpha p} \stackrel{\left(\begin{array}{c}
x \\
-x
\end{array}\right)}{\longrightarrow} X_{(\alpha p)^{+1} / \beta p}\right)=I_{X p \#, \beta / \alpha}
$$

from which $X p^{\#} \mathrm{~T}_{I(m)}=X \mathrm{~T}_{I(n)} p^{\#}$.

Next, starting with $X p^{\#}$, the object $X p^{\#} \mathrm{~T}_{J(m)}$ is calculated by means of $\left(J_{X p^{\#, \beta / \alpha}}\right)_{\beta / \alpha \in \bar{\Delta}_{m}^{\#}}$, whereas $X \mathrm{\top}_{J(n)} p^{\#}$ can be regarded as being calculated by means of $\left(J_{X, \beta p / \alpha p}\right)_{\beta / \alpha \in \bar{\Delta}_{m}^{\#}}$. But

$$
\begin{aligned}
J_{X, \beta p / \alpha p} & =\left(X_{\beta p / \alpha p} \stackrel{X_{\beta p / \alpha p^{\iota}}}{\longrightarrow} X_{\beta p / \alpha p} \mathrm{I}=X_{\beta p / \alpha p}^{+1} \mathrm{P} \stackrel{X_{\beta p / \alpha p}^{+1} \pi}{\longrightarrow} X_{\beta p / \alpha p}^{+1}\right) \\
& =J_{X p \#, \beta / \alpha},
\end{aligned}
$$

from which $X p^{\#} \mathrm{~T}_{J(m)}=X \mathrm{\top}_{J(n)} p^{\#}$.

Now

$$
X p^{\#} \mathrm{~T}_{I(m)} \stackrel{X p^{\#} \alpha_{I(m), J(m)}}{\sim} X p^{\#} \mathrm{~T}_{J(m)}
$$

is induced by $\left(I_{X p^{\#, \beta / \alpha}}\right)_{\beta / \alpha \in \bar{\Delta}_{m}^{\#}}$ and by $\left(J_{X p^{\#, \beta / \alpha}}\right)_{\beta / \alpha \in \bar{\Delta}_{m}^{\#}}$, whereas

$$
X \mathrm{~T}_{I(n)} p^{\# \underset{I(n), J(n)}{\sim} p^{\#}} \longrightarrow \mathrm{T}_{J(n)} p^{\#}
$$

can be regarded as being induced by $\left(I_{X, \beta p / \alpha p}\right)_{\beta / \alpha \in \bar{\Delta}_{m}^{\#}}$ and by $\left(J_{X, \beta p / \alpha p}\right)_{\beta / \alpha \in \bar{\Delta}_{m}^{\#}}$. We have just seen, however, that these pairs of tuples coincide.

Ad (**). By Proposition 4.5, we may assume $Y=X M$ for some $X \in$ Ob $\mathcal{F}^{\square}\left(\left(2 \bar{\Delta}_{n}\right)^{\#}\right)$. By Remark 4.1 , we have

$$
\begin{aligned}
& \left(X M \underline{\mathfrak{f}}_{n}\right) \vartheta_{n+1}=\left(X \tilde{\mathfrak{f}}_{n} M\right) \vartheta_{n+1} \quad=\left(X \tilde{\mathfrak{f}}_{n} \alpha_{I(n+1), J(n+1)}\right) M^{\prime \prime} \\
& \left(X M \vartheta_{2 n+1}\right) \underline{\mathfrak{f}}_{n}=\left(X \alpha_{I(2 n+1), J(2 n+1)} M^{\prime \prime}\right) \underline{\mathfrak{f}}_{n}=\left(X \alpha_{I(2 n+1), J(2 n+1)} \mathfrak{f}_{n}\right) M^{\prime \prime}
\end{aligned}
$$

so that it suffices to show that $X \tilde{\mathfrak{f}}_{n} \alpha_{I(n+1), J(n+1)}=X \alpha_{I(2 n+1), J(2 n+1)} \mathfrak{f}_{n}$.

Starting with $X \tilde{\mathfrak{f}}_{n}$, the object $X \tilde{\mathfrak{f}}_{n} \mathrm{\top}_{I(n+1)}$ is calculated by means of $\left(I_{X \tilde{\mathfrak{f}}_{n}, \beta / \alpha}\right)_{\beta / \alpha \in \overline{\rho \sqcup \Delta_{n}}}$; ; whereas $X \mathrm{~T}_{I(2 n+1)} \mathfrak{f}_{n}$ can be regarded as being calculated by means of the tuple of pure short exact sequences consisting of

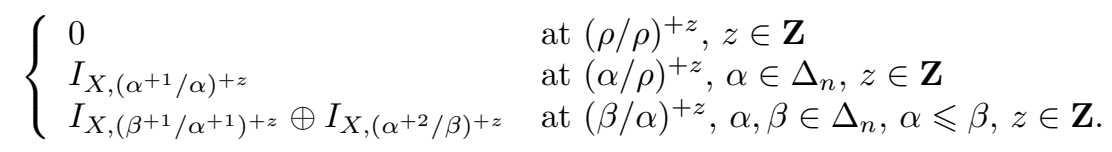


Homology, Homotopy and Applications, vol. 9(2), 2007

We have $I_{X \tilde{\mathfrak{f}}_{n},(\rho / \rho)^{+z}}=0$ for $z \in \mathbf{Z}$. For $\alpha \in \Delta_{n}$, we have

$$
I_{X \tilde{\mathfrak{f}}_{n}, \alpha / \rho}=\left(X_{\alpha^{+1} / \alpha} \stackrel{(x x)}{\longrightarrow} X_{\alpha^{+1} / \alpha^{+1}} \oplus X_{\alpha^{+2} / \alpha} \stackrel{\left(\begin{array}{c}
x \\
-x
\end{array}\right)}{\longrightarrow} X_{\alpha^{+2} / \alpha^{+1}}\right)=I_{X, \alpha^{+1} / \alpha},
$$

and accordingly at $(\alpha / \rho)^{+z}$ for $z \in \mathbf{Z}$. Moreover, for $\alpha, \beta \in \Delta_{n}$ with $\alpha \leqslant \beta$, we have

$$
\begin{aligned}
& I_{X \tilde{\mathfrak{f}}_{n}, \beta / \alpha}= \\
& \left(X_{\beta^{+1} / \alpha^{+1}} \oplus X_{\alpha^{+2} / \beta} \stackrel{\left(\begin{array}{cccc}
x & 0 & x & 0 \\
0 & x & 0 & -x
\end{array}\right)}{\longrightarrow} X_{\beta^{+1 / \beta}} \oplus X_{\beta^{+2} / \beta} \oplus X_{\alpha^{+3} / \alpha^{+1}} \oplus X_{\alpha^{+2} / \alpha^{+2}}\right. \\
& \left(\begin{array}{rr}
x & 0 \\
0 & -x \\
-x & 0 \\
0 & -x
\end{array}\right) \\
& \left.\stackrel{(0-x}{\longrightarrow} X_{\alpha^{+3} / \beta^{+1}} \oplus X_{\beta^{+2} / \alpha^{+2}}\right)
\end{aligned}
$$

and

$$
\begin{aligned}
& I_{X, \beta^{+1} / \alpha^{+1}} \oplus I_{X, \alpha^{+2} / \beta}=
\end{aligned}
$$

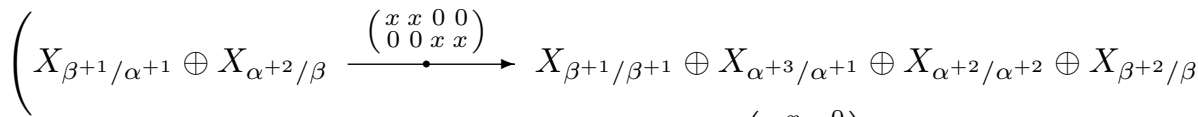

$$
\begin{aligned}
& \left(\begin{array}{rr}
x & 0 \\
-x & 0 \\
0 & x \\
0 & -x
\end{array}\right) \\
& \left.\stackrel{\sim}{\longrightarrow} X_{\alpha+3 / \beta^{+1}} \oplus X_{\beta^{+2} / \alpha^{+2}}\right)
\end{aligned}
$$

Accordingly at $(\beta / \alpha)^{+z}$ for $z \in \mathbf{Z}$.

Since there is an isomorphism from $I_{X \tilde{\mathfrak{f}}_{n}, \alpha / \rho}$ to $I_{X, \beta^{+1} / \alpha^{+1}} \oplus I_{X, \alpha^{+2} / \beta}$ that has identities on the first and on the third terms of the short exact sequences, completed by

$$
X_{\beta^{+1 / \beta+1}} \oplus X_{\beta^{+2} / \beta} \oplus X_{\alpha+3 / \alpha^{+1}} \oplus X_{\alpha^{+2} / \alpha^{+2}} \frac{\left(\begin{array}{cccc}
1 & 0 & 0 & 0 \\
0 & 0 & 0 & 1 \\
0 & 1 & 0 & 0 \\
0 & 0 & -1 & 0
\end{array}\right)}{\sim} \underset{X_{\beta^{+1} / \beta^{+1}} \oplus X_{\alpha^{+3} / \alpha^{+1}} \oplus X_{\alpha^{+2} / \alpha^{+2}}^{\longrightarrow} \oplus X_{\beta^{+2} / \beta}}{\longrightarrow}
$$

on the second terms, the characterisation in Lemma A.32.(1) shows that we end up with $X \tilde{\mathfrak{f}}_{n} \mathrm{~T}_{I(n+1)}=X \mathrm{~T}_{I(2 n+1)} \mathfrak{f}_{n}$.

Starting with $X \tilde{\mathfrak{f}}_{n}$, the object $X \tilde{\mathfrak{f}}_{n} \top_{J(n+1)}$ is calculated by means of

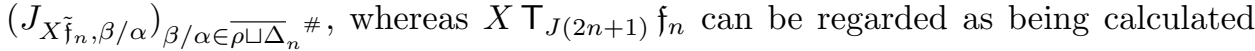
by means of the tuple of pure short exact sequences consisting of

$$
\begin{cases}0 & \text { at }(\rho / \rho)^{+z}, z \in \mathbf{Z} \\ J_{X,\left(\alpha^{+1} / \alpha\right)^{+z}} & \text { at }(\alpha / \rho)^{+z}, \alpha \in \Delta_{n}, z \in \mathbf{Z} \\ J_{X,\left(\beta^{+1} / \alpha^{+1}\right)^{+z}} \oplus J_{X,\left(\alpha^{+2} / \beta\right)^{+z}} & \text { at }(\beta / \alpha)^{+z}, \alpha, \beta \in \Delta_{n}, \alpha \leqslant \beta, z \in \mathbf{Z} .\end{cases}
$$

We have $J_{X \tilde{\mathfrak{F}}_{n},(\rho / \rho)^{+z}}=0$ for $z \in \mathbf{Z}$. For $\alpha \in \Delta_{n}$, we have

$$
\begin{aligned}
J_{X \tilde{\mathfrak{f}}_{n}, \alpha / \rho} & =\left(X_{\alpha^{+1} / \alpha} \stackrel{X_{\alpha+1 / \alpha} \iota}{\longrightarrow} X_{\alpha^{+1} / \alpha} \mathrm{I}=X_{\alpha^{+1} / \alpha}^{+1} \mathrm{P} \stackrel{X_{\alpha+1 / \alpha}^{+1} \pi}{\longrightarrow} X_{\alpha^{+1} / \alpha}^{+1}\right) \\
& =J_{X, \alpha^{+1} / \alpha},
\end{aligned}
$$


and accordingly at $(\alpha / \rho)^{+z}$ for $z \in \mathbf{Z}$.

Moreover, for $\alpha, \beta \in \Delta_{n}$ with $\alpha \leqslant \beta$, we have

$$
\begin{aligned}
& J_{X \tilde{\mathfrak{f}}_{n}, \beta / \alpha} \\
& =\left(X_{\beta^{+1} / \alpha^{+1}} \oplus X_{\alpha^{+2} / \beta} \quad \stackrel{\left(X_{\beta+1 / \alpha+1} \oplus X_{\alpha+2 / \beta}\right) \iota}{\longrightarrow}\right. \\
& \left(X_{\beta^{+1} / \alpha^{+1}} \oplus X_{\alpha^{+2} / \beta}\right) \mathrm{I}=\left(X_{\beta^{+1} / \alpha^{+1}} \oplus X_{\alpha^{+2} / \beta}\right)^{+1} \mathrm{P} \\
& \stackrel{\left(X_{\beta+1 / \alpha+1} \oplus X_{\alpha+2 / \beta}\right)^{+1} \pi}{1} \\
& \left.X_{\alpha^{+3} / \beta^{+1}} \oplus X_{\beta^{+2} / \alpha^{+2}}\right) \\
& =J_{X, \beta^{+1} / \alpha^{+1}} \oplus J_{X, \alpha^{+2} / \beta},
\end{aligned}
$$

and accordingly at $(\beta / \alpha)^{+z}$ for $z \in \mathbf{Z}$.

Hence we conclude that $X \tilde{\mathfrak{f}}_{n} \mathrm{~T}_{J(n+1)}=X \mathrm{~T}_{J(2 n+1)} \mathfrak{f}_{n}$.

Now

$$
X \tilde{\mathfrak{f}}_{n} \mathrm{\top}_{I(n+1)} \underset{\tilde{\mathfrak{f}}_{n} \alpha_{I(n+1), J(n+1)}}{\sim} X \tilde{\mathfrak{f}}_{n} \boldsymbol{\top}_{J(n+1)}
$$

is induced by $\left(I_{X \tilde{\mathfrak{f}}_{n}, \beta / \alpha}\right)_{\beta / \alpha \in \bar{\Delta}_{n+1}^{\#}}$ and by $\left(J_{X \tilde{\mathfrak{f}}_{n}, \beta / \alpha}\right)_{\beta / \alpha \in \bar{\Delta}_{n+1}^{\#}}$, whereas

$$
X \mathrm{~T}_{I(2 n+1)} \mathfrak{f}_{n} \stackrel{X \alpha_{I(2 n+1), J(2 n+1)} \mathfrak{f}_{n}}{\sim} X \mathrm{~T}_{J(2 n+1)} \mathfrak{f}_{n}
$$

can be regarded as being induced by the tuple consisting of

$$
\begin{cases}0 & \text { at }(\rho / \rho)^{+z}, z \in \mathbf{Z} \\ I_{X,\left(\alpha^{+1} / \alpha\right)^{+z}} & \text { at }(\alpha / \rho)^{+z}, \alpha \in \Delta_{n}, z \in \mathbf{Z} \\ I_{X,\left(\beta^{+1} / \alpha^{+1}\right)^{+z}} \oplus I_{X,\left(\alpha^{+2} / \beta\right)^{+z}} & \text { at }(\beta / \alpha)^{+z}, \alpha, \beta \in \Delta_{n}, \alpha \leqslant \beta, z \in \mathbf{Z} .\end{cases}
$$

and by $\left(J_{X \tilde{\mathfrak{f}}_{n}, \beta / \alpha}\right)_{\beta / \alpha \in \bar{\Delta}_{n+1}^{\#}}$.

Since the respective former tuples are isomorphic by a tuple of isomorphisms that has identities on the first and on the third term, and since the respective latter tuples are equal, the characterisation in Lemma A.32.(3) shows that in fact $X \tilde{\mathfrak{f}}_{n} \alpha_{I(n+1), J(n+1)}=X \alpha_{I(2 n+1), J(2 n+1)} \mathfrak{f}_{n}$.

Corollary 4.7. Let $\mathcal{E}$ be a Frobenius category. There exists a Heller triangulation on $(\underline{\underline{\mathcal{E}}}, \mathrm{T})$.

Proof. Let $\mathcal{B} \subseteq \mathcal{E}$ be the full subcategory of bijectives. The category $\mathcal{B}^{\text {ac }}$ is functorially Frobenius by Example A.6. Hence $\underline{\underline{\mathcal{E}}}=\underline{\mathcal{B}^{\text {ac }}}$, equipped with the complex shift $\mathrm{T}$, carries a Heller triangulation by virtue of Theorem 4.6.

\subsection{Exact functors induce strictly exact functors}

Proposition 4.8. Suppose given an exact functor

$$
\mathcal{F} \stackrel{F}{\longrightarrow} \tilde{\mathcal{F}}
$$


between functorial Frobenius categories $\mathcal{F}=(\mathcal{F}, \mathrm{T}, \mathrm{I}, \iota, \mathrm{P}, \pi)$ and $\tilde{\mathcal{F}}=(\tilde{\mathcal{F}}, \tilde{\mathrm{T}}, \tilde{\mathrm{I}}, \tilde{\iota}, \tilde{\mathrm{P}}, \tilde{\pi})$ that satisfies

$$
\begin{aligned}
F \tilde{\mathrm{T}} & =\mathrm{\top} F \\
F \tilde{\mathrm{I}} & =\mathrm{I} F \\
F \tilde{\mathrm{P}} & =\mathrm{P} F .
\end{aligned}
$$

Then the induced functor

$$
\underline{\mathcal{F}} \stackrel{F}{\longrightarrow} \underline{\tilde{\mathcal{F}}}
$$

is strictly exact with respect to the Heller triangulations introduced in Theorem 4.6.

Proof. Condition (1) of Definition 1.5.(iii) is satisfied. Condition (2) of loc. cit. holds since each morphism has a weak kernel that is sent to a weak kernel of its image; and dually. In fact, given a morphism represented by $X \stackrel{f}{\longrightarrow} Y$, the residue class of the kernel of $X \oplus Y \mathrm{P} \stackrel{\left(\begin{array}{c}f \\ Y \pi\end{array}\right)}{\longrightarrow} Y$ in $\mathcal{F}$, composed with $X \oplus Y \mathrm{P} \stackrel{\left(\begin{array}{l}1 \\ 0\end{array}\right)}{\longrightarrow} X$, is a weak kernel of the residue class of $X \stackrel{f}{\longrightarrow} Y$ by Lemma A.31 and Remark A.27. Since pure short exact sequences and bijectives are preserved by $F$, this weak kernel is preserved by $\underline{F}$.

Consider condition $(3)$ of loc. cit. Let $\vartheta$ resp. $\tilde{\vartheta}$ be the Heller triangulation on $\underline{\mathcal{F}}$ resp. on $\underline{\mathcal{F}}$ characterised as in Theorem 4.6 by

$$
\begin{aligned}
& \alpha_{I(n), J(n)} M^{\prime \prime}=M \vartheta_{n} \\
& \alpha_{\tilde{I}(n), \tilde{J}(n)} \tilde{M}^{\prime \prime}=\tilde{M} \tilde{\vartheta}_{n},
\end{aligned}
$$

where $\tilde{M}, \tilde{M}^{\prime}, \tilde{M}^{\prime \prime}, \tilde{I}(n)$ resp. $\tilde{J}(n)$ is defined over $\tilde{\mathcal{F}}$ as $M, M^{\prime}, M^{\prime \prime}, I(n) \operatorname{resp}$.

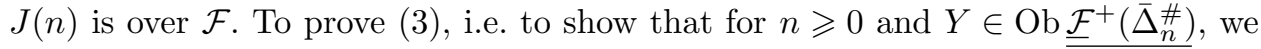
have

$$
\left(Y \vartheta_{n}\right) \underline{F}^{+}\left(\bar{\Delta}_{n}^{\#}\right)=\left(Y \underline{\underline{F}}^{+}\left(\bar{\Delta}_{n}^{\#}\right)\right) \tilde{\vartheta}_{n}
$$

we may assume by Proposition 4.5 that $Y=X M$ for some $X \in \operatorname{Ob} \mathcal{F}^{\square}\left(\bar{\Delta}_{n}^{\#}\right)$. Since

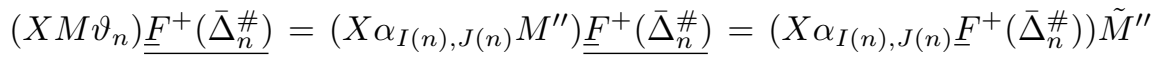

$$
\begin{aligned}
& \left(X M \underline{F}^{+}\left(\bar{\Delta}_{n}^{\#}\right)\right) \tilde{\vartheta}_{n}=\left(X F^{\square}\left(\bar{\Delta}_{n}^{\#}\right) \tilde{M}\right) \tilde{\vartheta}_{n} \quad=\left(X F^{\square}\left(\bar{\Delta}_{n}^{\#}\right) \alpha_{\tilde{I}(n), \tilde{J}(n)}\right) \tilde{M}^{\prime \prime},
\end{aligned}
$$

it suffices to show that $X \alpha_{I(n), J(n)} \underline{F}^{+}\left(\bar{\Delta}_{n}^{\#}\right)=X F^{\square}\left(\bar{\Delta}_{n}^{\#}\right) \alpha_{\tilde{I}(n), \tilde{J}(n)}$.

Starting with $X F^{\square}\left(\bar{\Delta}_{n}^{\#}\right)$, the object $X F^{\square}\left(\bar{\Delta}_{n}^{\#}\right) \mathrm{T}_{\tilde{I}(n)}$ is calculated by means of $\left(\tilde{I}_{X F^{\square}\left(\bar{\Delta}_{n}^{\#}\right), \beta / \alpha}\right)_{\beta / \alpha \in \bar{\Delta}_{n}^{\#}}$, whereas $X \mathrm{~T}_{\tilde{I}(n)} \underline{F}^{+}\left(\bar{\Delta}_{n}^{\#}\right)$ can be regarded as being calcu-

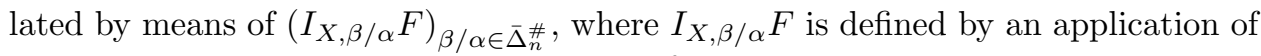
$F$ to all three terms and both morphisms of the pure short exact sequence $I_{X, \beta / \alpha}$. Since $F$ is additive, we get

$$
\left(\tilde{I}_{X F^{\square}\left(\bar{\Delta}_{n}^{\#}\right), \beta / \alpha}\right)_{\beta / \alpha \in \bar{\Delta}_{n}^{\#}}=\left(I_{X, \beta / \alpha} F\right)_{\beta / \alpha \in \bar{\Delta}_{n}^{\#},}
$$

from which $X F^{\square}\left(\bar{\Delta}_{n}^{\#}\right) \mathrm{\top}_{\tilde{I}(n)}=X \mathrm{\top}_{I(n)} \underline{F}^{+}\left(\bar{\Delta}_{n}^{\#}\right)$. 
Starting with $X F^{\square}\left(\bar{\Delta}_{n}^{\#}\right)$, the object $X F^{\square}\left(\bar{\Delta}_{n}^{\#}\right) \top_{\tilde{J}(n)}$ is calculated by means of

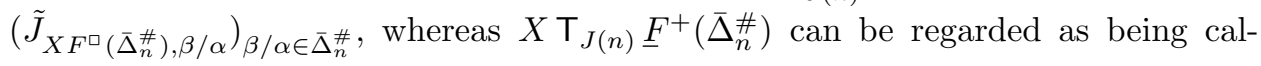

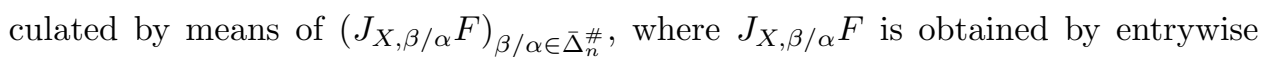
application of $F$. Since $F$ commutes with $\mathrm{P}$ and $\tilde{\mathrm{P}}$, and with $\mathrm{I}$ and $\tilde{\mathrm{I}}$, we get

$$
\left(\tilde{J}_{X F^{\square}\left(\bar{\Delta}_{n}^{\#}\right), \beta / \alpha}\right)_{\beta / \alpha \in \bar{\Delta}_{n}^{\#}}=\left(J_{X, \beta / \alpha} F\right)_{\beta / \alpha \in \bar{\Delta}_{n}^{\#}},
$$

from which $X F^{\square}\left(\bar{\Delta}_{n}^{\#}\right) \mathrm{\top}_{\tilde{J}(n)}=X \mathrm{\top}_{J(n)} \underline{F}^{+}\left(\bar{\Delta}_{n}^{\#}\right)$.

Moreover, since the defining pairs of tuples coincide, we now get

$$
X F^{\square}\left(\bar{\Delta}_{n}^{\#}\right) \alpha_{\tilde{I}(n), \tilde{J}(n)}=X \alpha_{I(n), J(n)} \underline{F}^{+}\left(\bar{\Delta}_{n}^{\#}\right) .
$$

Suppose given an exact functor

$$
\stackrel{E}{\longrightarrow} \tilde{\mathcal{E}}
$$

between Frobenius categories $\mathcal{E}$ and $\tilde{\mathcal{E}}$ that sends all bijective objects in $\mathcal{E}$ to bijective objects in $\tilde{\mathcal{E}}$. Let $\mathcal{B} \subseteq \mathcal{E}$ resp. $\tilde{\mathcal{B}} \subseteq \tilde{\mathcal{E}}$ be the respective subcategory of bijectives. We obtain an induced functor $\mathcal{B}^{\text {ac }} \stackrel{E^{\text {ac }}}{\longrightarrow} \tilde{\mathcal{B}}^{\text {ac }}$, inducing in turn a functor

$$
\underline{\underline{E}}:=\underline{E^{\mathrm{ac}}}: \underline{\underline{\mathcal{E}}}=\underline{\mathcal{B}^{\mathrm{ac}}} \longrightarrow \underline{\tilde{\mathcal{B}}^{\mathrm{ac}}}=\underline{\underline{\mathcal{E}}}
$$

modulo split acyclic complexes; see Example A.6.(2).

Corollary 4.9. The induced functor

$$
\underline{\underline{\mathcal{E}}} \stackrel{\underline{\underline{E}}}{\longrightarrow} \tilde{\underline{\mathcal{E}}}
$$

is strictly exact with respect to the Heller triangulations on $\underline{\underline{\mathcal{E}}}$ and on $\underline{\underline{\mathcal{E}}}$ introduced in Theorem 4.6 via the functorial Frobenius categories $\mathcal{B}^{\text {ac }}$ and $\tilde{\mathcal{B}}^{\text {ac }}$.

Proof. We may apply Proposition 4.8 to $(\mathcal{F} \stackrel{F}{\longrightarrow} \tilde{\mathcal{F}}):=\left(\mathcal{B}^{\text {ac }} \stackrel{E^{\text {ac }}}{\longrightarrow} \tilde{\mathcal{B}}^{\text {ac }}\right)$.

\section{Some quasicyclic categories}

In the definition of a Heller triangulated category, the categories $\mathcal{C}^{+}\left(\bar{\Delta}_{n}^{\#}\right)$ occur. Replacing this classical stable category by its stable counterpart, these turn out to be Heller triangulated themselves. So we can iterate. Cf. [2, Prop. 8.4].

Let $\mathcal{C}$ be a weakly abelian category. Let $n \geqslant 0$.

\subsection{The category $\mathcal{C}^{+}\left(\bar{\Delta}_{n}^{\#}\right)$ is Frobenius}

5.1.1 The Category $\mathcal{A}^{0}\left(\bar{\Delta}_{n}^{\#}\right)$ is Frobenius

We proceed in a slightly more general manner than necessary. We generalise the fact that the category of complexes $\mathcal{A}^{0}\left(\bar{\Delta}_{2}^{\#}\right)$ over an additive category $\mathcal{A}$ is a Frobenius category, to a category $\mathcal{A}^{0}\left(\bar{\Delta}_{n}^{\#}\right)$ for $n \geqslant 0$; see Lemma 5.2 below. Then we will specialise to our weakly abelian category $\mathcal{C}$ and pass to the full subcategory $\mathcal{C}^{+}\left(\bar{\Delta}_{n}^{\#}\right) \subseteq \mathcal{C}^{0}\left(\bar{\Delta}_{n}^{\#}\right)$; see Proposition 5.5 below. 


\subsubsection{Notation}

Let $\mathcal{A}$ be an additive category. Let $\mathcal{A}^{0}\left(\bar{\Delta}_{n}^{\#}\right)$ be the full subcategory of $\mathcal{A}\left(\bar{\Delta}_{n}^{\#}\right)$ defined by

$$
\operatorname{Ob} \mathcal{A}^{0}\left(\bar{\Delta}_{n}^{\#}\right):=\left\{X \in \operatorname{Ob} \mathcal{A}\left(\bar{\Delta}_{n}^{\#}\right): X_{\alpha / \alpha}=0 \text { and } X_{\alpha^{+1} / \alpha}=0 \text { for all } \alpha \in \bar{\Delta}_{n}\right\} .
$$

A sequence $X^{\prime} \stackrel{i}{\longrightarrow} X \stackrel{p}{\longrightarrow} X^{\prime \prime}$ in $\mathcal{A}^{0}\left(\bar{\Delta}_{n}^{\#}\right)$ is called pointwise split short exact if the sequence $X_{\xi}^{\prime} \stackrel{i_{\xi}}{\longrightarrow} X_{\xi} \stackrel{p_{\xi}}{\longrightarrow} X_{\xi}^{\prime \prime}$ is split short exact for all $\xi \in \bar{\Delta}_{n}^{\#}$. A morphism is called pointwise split monomorphic (resp. epimorphic) if it appears as a kernel (resp. cokernel) in a pointwise split short exact sequence.

The category $\mathcal{A}^{0}\left(\bar{\Delta}_{n}^{\#}\right)$ carries an outer shift functor $X \longmapsto[X]^{+1}$, where $[X]_{\beta / \alpha}^{+1}:=X_{(\beta / \alpha)^{+1}}=X_{\alpha^{+1} / \beta}$ for $\beta / \alpha \in \bar{\Delta}_{n}^{\#}$.

Recall that $\mathcal{A}$, together with the set of split short exact sequences, is an exact category by Example A.3. So the additive category $\mathcal{A}^{0}\left(\bar{\Delta}_{n}^{\#}\right)$, equipped with the set of pointwise split short exact sequences as pure short exact sequences, is an exact category by Example A.4.

Given $\beta / \alpha, \delta / \gamma \in \bar{\Delta}_{n}^{\#}$, we write $\beta / \alpha \lessdot \delta / \gamma$ if $\alpha<\gamma$ and $\beta<\delta$.

Given $A \in \operatorname{Ob} \mathcal{A}$ and $\beta / \alpha \in \bar{\Delta}_{n}^{\#}$, we denote by $A_{] \alpha, \beta]}$ the object in $\mathcal{A}^{0}\left(\bar{\Delta}_{n}^{\#}\right)$ consisting of identical morphisms wherever possible and having

$$
\left(A_{] \alpha, \beta]}\right)_{\delta / \gamma}:= \begin{cases}A & \text { if } \alpha / \beta^{-1} \lessdot \delta / \gamma \leqslant \beta / \alpha \\ 0 & \text { else }\end{cases}
$$

for $\delta / \gamma \in \bar{\Delta}_{n}^{\#}$. Such an object is called an extended interval. Intuitively, it is a rectangle with upper right corner at $\beta / \alpha$, and as large as possible in $\mathcal{A}^{0}\left(\bar{\Delta}_{n}^{\#}\right)$.

Let $\mathcal{A}^{+ \text {,split }}\left(\bar{\Delta}_{n}^{\#}\right)$ be the full subcategory of $\mathcal{A}^{0}\left(\bar{\Delta}_{n}^{\#}\right)$ consisting of objects isomorphic to summands of objects of the form

$$
\bigoplus_{\beta / \alpha \in \bar{\Delta}_{n}^{\#}}\left(A_{\beta / \alpha}\right)_{] \alpha, \beta]},
$$

where $A_{\beta / \alpha} \in \mathrm{Ob} \mathcal{A}$ for $\beta / \alpha \in \bar{\Delta}_{n}^{\#}$. This direct sum exists since it is a finite direct sum at each $\delta / \gamma \in \bar{\Delta}_{n}^{\#}$. Concerning the notation $\mathcal{A}^{+ \text {,split }}\left(\bar{\Delta}_{n}^{\#}\right)$, see also Remark 5.3 below.

\subsubsection{THE PERIODIC CASE}

Let $\mathcal{A}^{\prime}$ be an additive category, equipped with a graduation shift automorphism $X \longmapsto X[+1]$. We write $X \longmapsto X[m]$ for its $m$ th iteration, where $m \in \mathbf{Z}$.

By entrywise application, there is also a graduation shift on $\mathcal{A}^{\prime 0}\left(\bar{\Delta}_{n}^{\#}\right)$, likewise denoted by $X \longmapsto X[+1]$.

As in $\S 2.5 .3$, we define the subcategory $\mathcal{A}^{\prime 0, \text { periodic }}\left(\bar{\Delta}_{n}^{\#}\right) \subseteq \mathcal{A}^{\prime 0}\left(\bar{\Delta}_{n}^{\#}\right)$ to consist of the morphisms $X \stackrel{f}{\longrightarrow} Y$ in $\mathcal{A}^{\prime 0}\left(\bar{\Delta}_{n}^{\#}\right)$ that satisfy

$$
(X[+1] \stackrel{f[+1]}{\longrightarrow} Y[+1])=\left([X]^{+1} \stackrel{[f]^{+1}}{\longrightarrow}[Y]^{+1}\right)
$$

So the subcategory $\mathcal{A}^{\prime 0, \text { periodic }}\left(\bar{\Delta}_{n}^{\#}\right) \subseteq \mathcal{A}^{\prime 0}\left(\bar{\Delta}_{n}^{\#}\right)$ is not full in general. 
Given $A \in \mathrm{Ob} \mathcal{A}^{\prime}$ and $0 \leqslant i \leqslant j \leqslant n$, we denote by $A_{] i, j]}$ the object in $\mathcal{A}^{\prime 0}\left(\bar{\Delta}_{n}^{\#}\right)$ consisting only of zero and identical morphisms and having

$$
\left(A_{] i, j]}\right)_{\delta / \gamma}:= \begin{cases}A[m] & \text { if }\left(i / j^{-1}\right)^{+m} \lessdot \delta / \gamma \leqslant(j / i)^{+m} \text { for some } m \in \mathbf{Z} \\ 0 & \text { else }\end{cases}
$$

for $\delta / \gamma \in \bar{\Delta}_{n}^{\#}$. Intuitively, it is a rectangle with upper right corner at $j / i$, and as large as possible in $\mathcal{A}^{\prime 0 \text {,periodic }}\left(\bar{\Delta}_{n}^{\#}\right)$, repeated $\mathbf{Z}$-periodically up to the corresponding graduation shift.

Let $\mathcal{A}^{\prime+\text {,split,periodic }}\left(\bar{\Delta}_{n}^{\#}\right)$ be the full subcategory of $\mathcal{A}^{\prime 0 \text {,periodic }}\left(\bar{\Delta}_{n}^{\#}\right)$ consisting of objects isomorphic to summands of objects of the form

$$
\bigoplus_{0 \leqslant i \leqslant j \leqslant n}\left(A_{j, i}\right)_{] i, j]},
$$

where $A_{j, i} \in \mathrm{Ob} \mathcal{A}^{\prime}$ for $0 \leqslant i \leqslant j \leqslant n$. Such an object is called a periodic extended interval.

Lemma 5.1. The category $\mathcal{A}^{\prime 0, \text { periodic }}\left(\bar{\Delta}_{n}^{\#}\right)$, equipped with the pointwise split short exact sequences, is a Frobenius category, having $\mathcal{A}^{\prime+\text {,split,periodic }}\left(\bar{\Delta}_{n}^{\#}\right)$ as its subcategory of bijectives.

Proof. By duality, it suffices to show that the following assertions $(1,2)$ hold.

(1) The object $A_{i i, j]}$ is injective in $\mathcal{A}^{\prime 0, \text { periodic }}\left(\bar{\Delta}_{n}^{\#}\right)$ for any $A \in \mathrm{Ob} \mathcal{A}^{\prime}$ and any $0 \leqslant i \leqslant j \leqslant n$.

(2) For each object of $\mathcal{A}^{\prime 0 \text {,periodic }}\left(\bar{\Delta}_{n}^{\#}\right)$, there exists a pure monomorphism into an object of $\mathcal{A}^{\prime+, \text { split,periodic }}\left(\bar{\Delta}_{n}^{\#}\right)$.

Ad (1). Note that we have an adjunction isomorphism

$$
\begin{aligned}
& \mathcal{A}^{\prime 0, \text { periodic }}\left(\bar{\Delta}_{n}^{\#}\right)\left(X, A_{] i, j]}\right) \stackrel{\sim}{\longrightarrow} \mathcal{A}^{\prime}\left(X_{j / i}, A\right) \\
& f \longmapsto f_{j / i},
\end{aligned}
$$

where $X \in \operatorname{Ob} \mathcal{A}^{\prime 0, \text { periodic }}\left(\bar{\Delta}_{n}^{\#}\right)$. Suppose given a pure monomorphism $A_{] i, j]} \rightarrow X$ for some $A \in \mathrm{Ob} \mathcal{A}^{\prime}$. Let $\left(A \longrightarrow X_{j / i} \longrightarrow A\right)=1_{A}$. Let $X \longrightarrow A_{] i, j]}$ correspond to $X_{j / i} \longrightarrow A$. The composition $\left(A_{] i, j]} \rightarrow X \longrightarrow A_{] i, j]}\right)$ restricts to $1_{A}$ at $j / i$, hence equals $1_{\left.A_{i, j]}\right]}$.

Ad (2). Suppose given $X \in \operatorname{Ob} \mathcal{A}^{\prime 0, \text { periodic }}\left(\bar{\Delta}_{n}^{\#}\right)$. Given $0 \leqslant i \leqslant j \leqslant n$, we let

$$
X \stackrel{X s_{j / i}}{\longrightarrow}\left(X_{j / i}\right)_{] i, j]}
$$

be the morphism corresponding to $1_{X_{j / i}}$ by adjunction, which is natural in $X$. Collecting these morphisms yields a morphism

$$
X \stackrel{X s}{\longrightarrow} \bigoplus_{0 \leqslant i \leqslant j \leqslant n}\left(X_{j / i}\right)_{] i, j]},
$$

which is pointwise split monomorphic since at $j / i$, its component $X_{j / i} \longrightarrow X_{j / i}$ is an identity. 


\subsubsection{The General CASE}

Lemma 5.2. The category $\mathcal{A}^{0}\left(\bar{\Delta}_{n}^{\#}\right)$, equipped with the pointwise split short exact sequences, is a Frobenius category, having $\mathcal{A}^{+, \text {split }}\left(\bar{\Delta}_{n}^{\#}\right)$ as its subcategory of bijectives.

Proof. To prove that $\mathcal{A}^{0}\left(\bar{\Delta}_{n}^{\#}\right)$ is a Frobenius category, we more precisely claim that $\mathcal{A}^{+, \text {split }}\left(\bar{\Delta}_{n}^{\#}\right)$ is a sufficiently large category of bijective objects in the exact category $\mathcal{A}^{0}\left(\bar{\Delta}_{n}^{\#}\right)$.

Abbreviate $\mathcal{A}^{\mathbf{Z}}:=\mathcal{A}(\dot{\mathbf{Z}})$, where $\dot{\mathbf{Z}}$ denotes the discrete category with $\mathrm{Ob} \dot{\mathbf{Z}}=\mathbf{Z}$ and only identical morphisms. The category $\mathcal{A}^{\mathbf{Z}}$ carries the graduation shift automorphism

$$
\begin{aligned}
\mathcal{A}^{\mathbf{Z}} \stackrel{\sim}{\longrightarrow} \mathcal{A}^{\mathbf{Z}} & \\
(X \stackrel{f}{\longrightarrow} Y) & \longmapsto(X[+1] \stackrel{f[+1]}{\longrightarrow} Y[+1]):=\left(X_{i+1} \stackrel{f_{i+1}}{\longrightarrow} Y_{i+1}\right)_{i \in \mathbf{Z}} .
\end{aligned}
$$

We have an isomorphism of categories

$$
\begin{aligned}
\mathcal{A}^{+}\left(\bar{\Delta}_{n}^{\#}\right) & \stackrel{\Phi}{\sim}\left(\mathcal{A}^{\mathbf{Z}}\right)^{+, \text {periodic }}\left(\bar{\Delta}_{n}^{\#}\right) \\
X & \longmapsto\left(\left(X_{(\beta / \alpha)^{+i}}\right)_{i \in \mathbf{Z}}\right)_{\beta / \alpha \in \bar{\Delta}_{n}^{\#}} \\
\left(\left(Y_{\beta / \alpha}\right)_{0}\right)_{\beta / \alpha \in \bar{\Delta}_{n}^{\#}} & \longmapsto Y .
\end{aligned}
$$

Both categories are exact when equipped with pointwise split short exact sequences, and $\Phi$ and $\Phi^{-1}$ are exact functors. We then have $\mathcal{A}^{+, \text {split }}\left(\bar{\Delta}_{n}^{\#}\right) \Phi=$ $\left(\mathcal{A}^{\mathbf{Z}}\right)^{+, \text {split,periodic }}\left(\bar{\Delta}_{n}^{\#}\right)$.

Putting $\mathcal{A}^{\prime}:=\mathcal{A}^{\mathbf{Z}}$, the result follows by Lemma 5.1.

If $\mathcal{A}=\mathcal{C}$ is a weakly abelian category, we have two definitions of $\mathcal{C}^{+ \text {,split }}\left(\bar{\Delta}_{n}^{\#}\right)$.

The first one, given in 1.2.1.1, defines this category as a full subcategory of $\mathcal{C}^{+}\left(\bar{\Delta}_{n}^{\#}\right)$ containing those diagrams in which all morphisms are split.

The second one, just given, defines this category as a full subcategory of $\mathcal{C}^{0}\left(\bar{\Delta}_{n}^{\#}\right)$ containing, up to isomorphism, summands of direct sums of extended intervals.

Remark 5.3. If $\mathcal{A}=\mathcal{C}$ is a weakly abelian category, then the two aforementioned definitions of $\mathcal{C}^{+, \text {split }}\left(\bar{\Delta}_{n}^{\#}\right)$ coincide.

Proof. First, we notice that an extended interval lies in $\mathcal{C}^{+}\left(\bar{\Delta}_{n}^{\#}\right)$, and that all its diagram morphisms are split.

It remains to be shown that an object in $\mathcal{C}^{+}\left(\bar{\Delta}_{n}^{\#}\right)$, all of whose diagram morphisms are split, is, up to isomorphism, a summand of a direct sum of extended intervals.

Passing to $\left(\mathcal{C}^{\mathbf{Z}}\right)^{+, \text {periodic }}\left(\bar{\Delta}_{n}^{\#}\right)$, we have to show that an object $X \in$ $\operatorname{Ob}\left(\mathcal{C}^{\mathbf{Z}}\right)^{+ \text {,periodic }}\left(\bar{\Delta}_{n}^{\#}\right)$ all of whose diagram morphisms are split, is, up to isomorphism, a summand of a direct sum of periodic extended intervals.

By Lemma A.25, applied to the abelian Frobenius category given by the Freyd category of $\mathcal{C}^{\mathbf{Z}}$, the object $\left.X\right|_{\dot{\Delta}_{n}} \in \operatorname{Ob}^{\mathbf{Z}}\left(\dot{\Delta}_{n}\right)$ is isomorphic to a summand of a finite direct sum of intervals. Hence, by Lemma 2.11, the object $X$ is isomorphic to a summand of a finite direct sum of images of intervals under $\bar{S}$, i.e. of periodic extended intervals, as required. 


\subsubsection{The Subcategory $\mathcal{C}^{+}\left(\bar{\Delta}_{n}^{\#}\right) \subseteq \mathcal{C}^{0}\left(\bar{\Delta}_{n}^{\#}\right)$}

Recall that $\mathcal{C}$ is a weakly abelian category.

Lemma 5.4. Suppose given a pure short exact sequence

$$
X^{\prime} \rightarrow X \rightarrow X^{\prime \prime}
$$

in $\mathcal{C}^{0}\left(\bar{\Delta}_{n}^{\#}\right)$. If two out of the three objects $X^{\prime}, X$ and $X^{\prime \prime}$ are in $\mathcal{C}^{+}\left(\bar{\Delta}_{n}^{\#}\right)$, so is the third.

Proof. For an object $X \in \mathrm{ObC}^{0}\left(\bar{\Delta}_{n}\right)$ to lie in $\mathrm{ObC}^{+}\left(\bar{\Delta}_{n}\right)$, it suffices to know that the complex

$$
\begin{aligned}
& X(\alpha, \beta, \gamma):= \\
& \left(\cdots \longrightarrow X_{\beta / \gamma^{-1}} \longrightarrow X_{\beta / \alpha} \longrightarrow X_{\gamma / \alpha} \longrightarrow X_{\gamma / \beta} \longrightarrow X_{\alpha^{+1} / \beta} \longrightarrow \cdots\right)
\end{aligned}
$$

is acyclic in $\hat{\mathcal{C}}$ for all $\alpha, \beta, \gamma \in \bar{\Delta}_{n}$ with $\alpha \leqslant \beta \leqslant \gamma \leqslant \alpha^{+1}$; which is true, as we take from Lemma A.17; see Remark A.27.

Now the long exact homology sequence, applied in $\hat{\mathcal{C}}$ to the short exact sequence $X^{\prime}(\alpha, \beta, \gamma) \longrightarrow X(\alpha, \beta, \gamma) \longrightarrow X^{\prime \prime}(\alpha, \beta, \gamma)$ of complexes, shows that if two of these complexes are acyclic, so is the third.

\section{Proposition 5.5.}

(1) The category $\mathcal{C}^{+}\left(\bar{\Delta}_{n}^{\#}\right)$, equipped with the pointwise split short exact sequences as pure short exact sequences, is a Frobenius category, having $\mathcal{C}^{+, \operatorname{split}}\left(\bar{\Delta}_{n}^{\#}\right)$ as its subcategory of bijectives. Hence its stable category $\underline{\mathcal{C}^{+}\left(\bar{\Delta}_{n}^{\#}\right)}$ is equivalent to its classical stable category $\underline{\mathcal{C}^{+}\left(\bar{\Delta}_{n}^{\#}\right)}=\mathcal{C}^{+}\left(\bar{\Delta}_{n}^{\#}\right) / \mathcal{C}^{+, \operatorname{split}}\left(\bar{\Delta}_{n}^{\#}\right)$. So both $\underline{\underline{\mathcal{C}^{+}\left(\bar{\Delta}_{n}^{\#}\right)}}$ and $\underline{\mathcal{C}^{+}\left(\bar{\Delta}_{n}^{\#}\right)}$ are weakly abelian.

(2) Suppose $\mathcal{C}$ to be equipped with an automorphism $X \longmapsto X^{+1}$. The category $\mathcal{C}^{+ \text {,periodic }}\left(\bar{\Delta}_{n}^{\#}\right)$ (see $\left.\oint 2.5 .3\right)$, equipped with the pointwise split short exact sequences as pure short exact sequences, is an additively functorial Frobenius category, having $\mathcal{C}^{+ \text {,split,periodic }}\left(\bar{\Delta}_{n}^{\#}\right)$ as its subcategory of bijectives.

We remark that $\mathcal{C}^{+}\left(\bar{\Delta}_{n}^{\#}\right)$ appears in Definition 1.5 .

Proof. Ad (1). To prove that $\mathcal{C}^{+}\left(\bar{\Delta}_{n}^{\#}\right)$ is an exact category, it remains to be shown, in view of Lemma 5.2 and of $\S$ A.2.2, that a pure short exact sequence in $\mathcal{C}^{0}\left(\bar{\Delta}_{n}^{\#}\right)$ that has the first and the third term in $\mathrm{ObC}^{+}\left(\bar{\Delta}_{n}^{\#}\right)$, has the second term in $\mathrm{Ob}^{+}\left(\bar{\Delta}_{n}^{\#}\right)$, too. This follows by Lemma 5.4 .

To prove that $\mathcal{C}^{+}\left(\bar{\Delta}_{n}^{\#}\right)$ is Frobenius, we may use that $\mathcal{C}^{0}\left(\bar{\Delta}_{n}^{\#}\right)$ is Frobenius, with the bijective objects already lying in $\mathcal{C}^{+}\left(\bar{\Delta}_{n}^{\#}\right)$, thus being a fortiori bijective with respect to $\mathcal{C}^{+}\left(\bar{\Delta}_{n}^{\#}\right)$. By duality, it remains to be shown that the kernel of a pointwise split epimorphism of a bijective object to a given object in $\mathcal{C}^{+}\left(\bar{\Delta}_{n}^{\#}\right)$ is again in $\mathcal{C}^{+}\left(\bar{\Delta}_{n}^{\#}\right)$, thus showing that this epimorphism is actually pure in $\mathcal{C}^{+}\left(\bar{\Delta}_{n}^{\#}\right)$. This follows by Lemma 5.4 .

Ad (2). In view of Lemma 5.1, this follows as (1). 
I do not know whether $\mathcal{C}^{+, \vartheta=1}\left(\bar{\Delta}_{n}^{\#}\right)$ is Frobenius. It seems doubtful, since this question is reminiscent of the example of A. NEEMAN which shows that the mapping cone of a morphism of distinguished triangles in the sense of Verdier need not be distinguished [24, p. 234].

\subsubsection{Two EXAMPLES}

Suppose $\mathcal{C}$ to be equipped with an automorphism $X \longmapsto X^{+1}$.

The category $\mathcal{C}^{+, \text {periodic }}\left(\bar{\Delta}_{n}^{\#}\right)$ being a Frobenius category by Proposition 5.5.(2), its classical stable category $\mathcal{C}^{+ \text {,periodic }}\left(\bar{\Delta}_{n}^{\#}\right)$ carries a Heller operator, defined on $X \in$ $\operatorname{Ob} \mathcal{C}^{+, \text {periodic }}\left(\bar{\Delta}_{n}^{\#}\right)$ as the kernel of $B \longrightarrow X$, where $B \in \operatorname{ObC}^{+, \text {split,periodic }}\left(\bar{\Delta}_{n}^{\#}\right)$. As examples, we calculate the Heller operator for $n \in\{2,3\}$ on periodic $n$-pretriangles.

Suppose $n=2$. Let $X \in \operatorname{Ob} \mathcal{C}^{+ \text {,periodic }}\left(\bar{\Delta}_{2}^{\#}\right)$ be a periodic 2-pretriangle. We obtain the diagram shown in Figure 5.1.

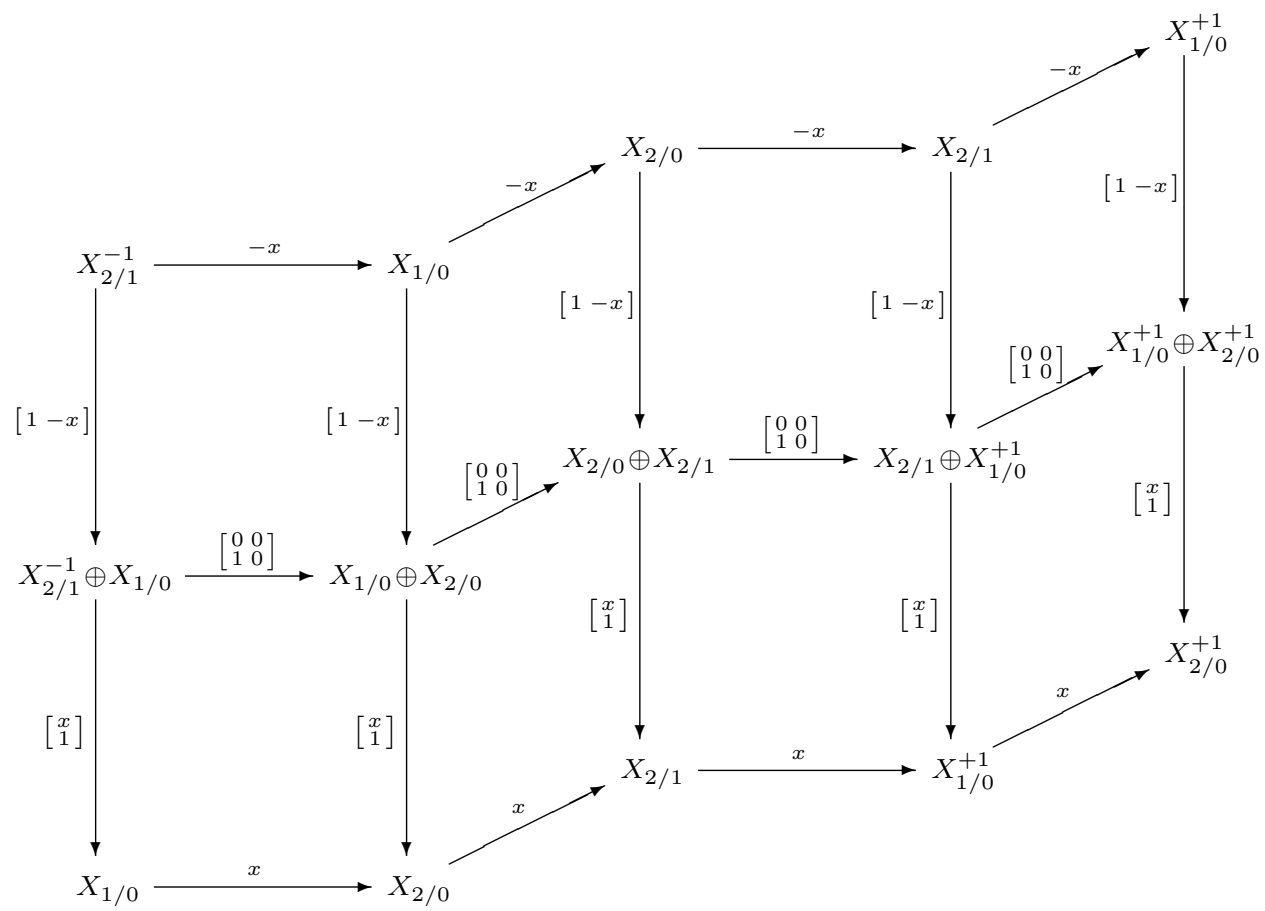

Figure 5.1

In particular, if $X$ is a 2 -triangle, i.e. an object of $\mathcal{C}^{+, \vartheta=1}\left(\bar{\Delta}_{2}^{\#}\right)$, then this Heller shift of $X$ is also a 2-triangle; see Lemma 3.5.

Suppose $n=3$. Let $X \in \mathrm{ObC}^{+ \text {,periodic }}\left(\bar{\Delta}_{3}^{\#}\right)$ be a periodic 3 -pretriangle. We obtain the diagram shown in Figure 5.2.

If $X$ is a 3 -triangle, i.e. an object $\mathcal{C}^{+, \vartheta=1}\left(\bar{\Delta}_{3}^{\#}\right)$, I do not know whether this Heller shift of $X$ is again a 3 -triangle. 
Homology, Homotopy and Applications, vol. 9(2), 2007

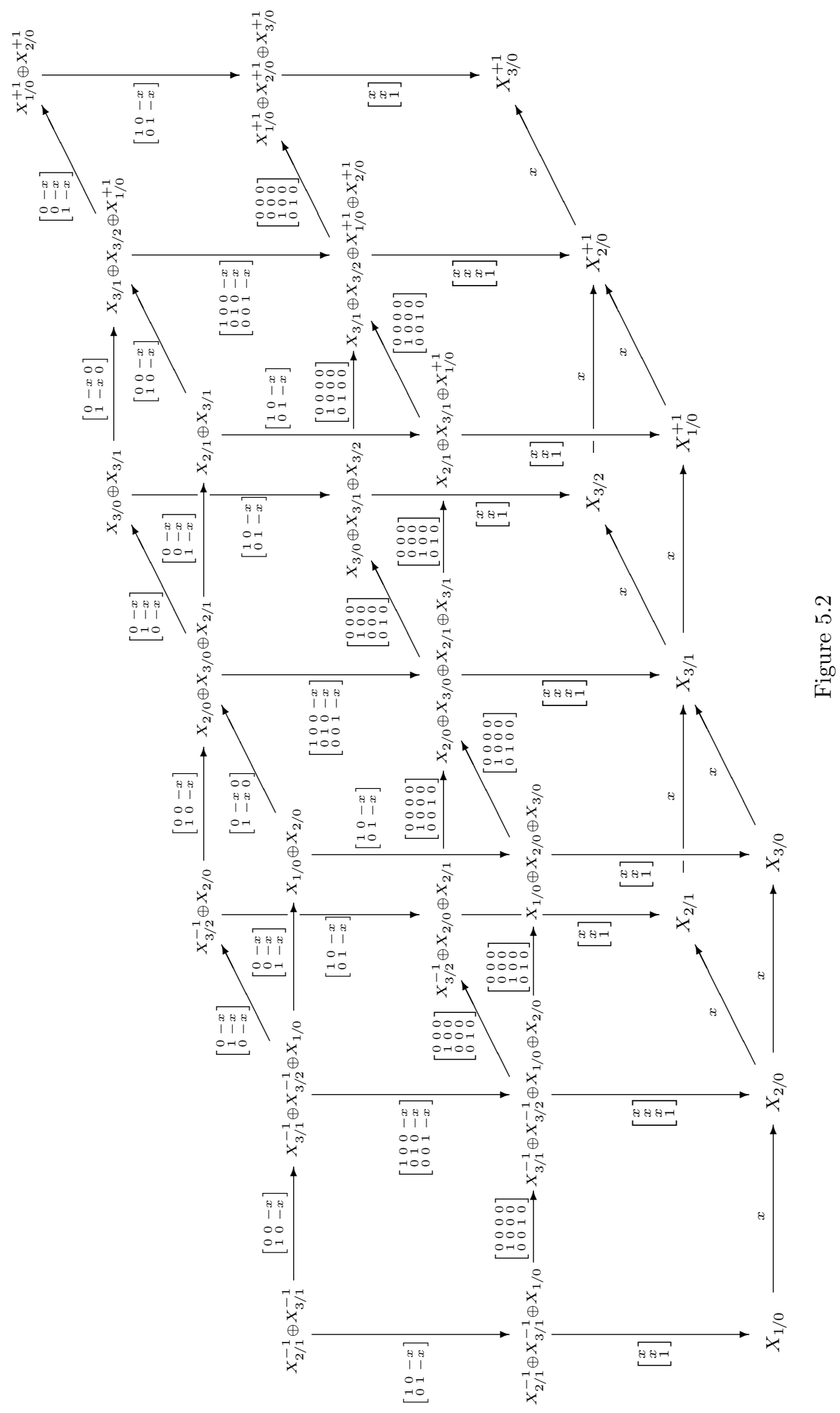




\subsection{A quasicyclic category}

The category of quasicyclic categories is defined to be the category of contravariant functors from $\overline{\boldsymbol{\Delta}}^{\circ}$ to the (1-)category (Cat) of categories. Recall that we have a functor $\boldsymbol{\Delta} \longrightarrow \overline{\boldsymbol{\Delta}}, \Delta_{n} \longmapsto \bar{\Delta}_{n}$ that allows a quasicyclic set to be restricted to its underlying simplicial set.

Given a category $\mathcal{U}$, we denote by Iso $\mathcal{U} \subseteq \mathcal{U}$ its subcategory consisting of isomorphisms. Given a functor $\mathcal{U} \stackrel{U}{\longrightarrow} \mathcal{U}^{\prime}$, we denote by Iso $F:$ Iso $\mathcal{U} \longrightarrow$ Iso $\mathcal{U}^{\prime}$ the induced functor.

We define

$$
\begin{aligned}
& \bar{\Delta} \stackrel{\text { qcyc. } \mathcal{C}}{\longrightarrow}(\text { Cat }) \\
& \left(\bar{\Delta}_{n} \stackrel{f}{\longleftarrow} \bar{\Delta}_{n}\right) \longmapsto\left(\text { Iso } \mathcal{C}^{+}\left(\bar{\Delta}_{n}^{\#}\right) \stackrel{\text { Iso } \mathcal{C}^{+}\left(f^{\#}\right)}{\longrightarrow} \text { Iso } \mathcal{C}^{+}\left(\bar{\Delta}_{m}^{\#}\right)\right) .
\end{aligned}
$$

More intuitively written, qcyc. $\mathcal{C}:=$ Iso $\mathcal{C}^{+}\left(\bar{\Delta}_{\bullet}^{\#}\right)$. Note that $\operatorname{qcyc}_{0} \mathcal{C}$ consists only of zero-objects.

A strictly exact functor $\mathcal{C} \stackrel{F}{\longrightarrow} \mathcal{C}^{\prime}$ induces a functor $\mathcal{C}^{+}\left(\bar{\Delta}_{n}^{\#}\right) \stackrel{F^{+}\left(\bar{\Delta}_{n}^{\#}\right)}{\longrightarrow}$ $\mathcal{C}^{\prime+}\left(\bar{\Delta}_{n}^{\#}\right)$ for $n \geqslant 0$, and thus a morphism

$$
\text { qcyc. }_{\bullet} \stackrel{\text { qcyc}_{\bullet} F}{\longrightarrow} \text { qcyc. }_{\bullet} \mathcal{C}^{\prime}
$$

of quasicyclic categories.

As variants, we mention

$$
\begin{aligned}
\text { qcyc }_{\bullet}^{\text {periodic }} \mathcal{C} & :=\operatorname{Iso} \mathcal{C}^{+, \text {periodic }}\left(\bar{\Delta}_{\bullet}^{\#}\right) \\
\text { qcyc }_{\bullet}^{\vartheta=1} \mathcal{C} & :=\operatorname{Iso} \mathcal{C}^{+, \vartheta=1}\left(\bar{\Delta}_{\bullet}^{\#}\right) .
\end{aligned}
$$

\subsection{A biquasicyclic category}

As an attempt in the direction described in [30, p. 330], we define a first step of an "iteration" of the construction qcyc. $\mathcal{C}$.

By Proposition 5.5, we may form the category $\mathcal{C}^{+}\left(\bar{\Delta}_{n}^{\#}\right)^{+}\left(\bar{\Delta}_{m}^{\#}\right)$. Note that a morphism $\bar{\Delta}_{m} \stackrel{f}{\frac{f}{\Delta}} \bar{\Delta}_{m^{\prime}}$ of periodic linearly ordered sets induces a functor $\underline{\underline{\mathcal{C}^{+}\left(\bar{\Delta}_{n}^{\#}\right)^{+}}}\left(f^{\#}\right)$ in the second variable.

By Lemma 4.8, a morphism $\bar{\Delta}_{n} \stackrel{g}{ } \bar{\Delta}_{n^{\prime}}$ of periodic linearly ordered sets induces a strictly exact functor $\underline{\mathcal{C}^{+}\left(g^{\#}\right)}$, and so a functor $\underline{\mathcal{C}^{+}\left(g^{\#}\right)^{+}}\left(\bar{\Delta}_{m}^{\#}\right)$ in the first variable for $m \geqslant 0$.

The functors induced by $f$ and by $g$ commute.

We may define

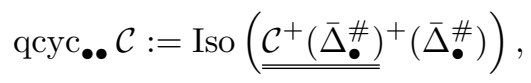

which yields a biquasicyclic category, i.e. a functor from $\bar{\Delta}^{\circ} \times \bar{\Delta}^{\circ}$ to the (1-)category (Cat) of categories. 
Homology, Homotopy and Applications, vol. 9(2), 2007

By Lemma 4.8 , a strictly exact functor $\mathcal{C} \stackrel{F}{\longrightarrow} \mathcal{C}^{\prime}$ induces a functor

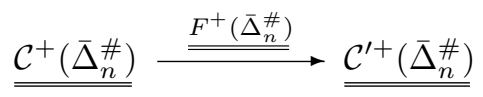

for $n \geqslant 0$, and thus a morphism

$$
\mathrm{qcyc}_{\bullet} \mathcal{C} \stackrel{\mathrm{qcyc}_{\bullet} F}{\longrightarrow} \mathrm{qcyc}_{\bullet} \mathcal{C}^{\prime}
$$

of quasicyclic categories.

As variants, we mention

$$
\begin{aligned}
\operatorname{qcyc}_{\bullet \bullet}^{\text {periodic }} \mathcal{C} & :=\operatorname{Iso}\left({\underline{\underline{\mathcal{C}^{+}\left(\bar{\Delta}_{\bullet}^{\#}\right)}}}^{+, \operatorname{periodic}}\left(\bar{\Delta}_{\bullet}^{\#}\right)\right) \\
\operatorname{qcyc}_{\bullet \bullet}^{\vartheta=1} \mathcal{C} & \left.:=\operatorname{Iso}\left(\underline{\underline{\mathcal{C}^{+}\left(\bar{\Delta}_{\bullet}^{\#}\right.}}\right)^{+, \vartheta=1}\left(\bar{\Delta}_{\bullet}^{\#}\right)\right) .
\end{aligned}
$$

Cf. Remark 3.3.

This procedure can be iterated to obtain triquasicyclic categories etc.

\section{Appendix A. Some general lemmata}

This appendix is a tool kit consisting of folklore lemmata (with proof) and known results (mainly without proof). We do not claim originality.

\section{A.1. An additive lemma}

Let $\mathcal{A}$ and $\mathcal{B}$ be additive categories, and let $\mathcal{A} \stackrel{F}{\longrightarrow} \mathcal{B}$ be a full and dense additive functor. Let $\mathcal{N} \subseteq \mathcal{B}$ be a full additive subcategory. Let $\mathcal{M} \subseteq \mathcal{A}$ be the full subcategory determined by

$$
\mathrm{Ob} \mathcal{M}:=\{A \in \mathrm{Ob} \mathcal{A}: A F \text { is isomorphic to an object of } \mathcal{N}\} .
$$

Lemma A.1. Suppose that for each morphism $A \stackrel{a_{0}}{\longrightarrow} A^{\prime}$ in $\mathcal{A}$ such that $a_{0} F=0$, there exists a factorisation

$$
\left(A \stackrel{a_{0}}{\longrightarrow} A^{\prime}\right)=\left(A \stackrel{a_{0}^{\prime}}{\longrightarrow} M_{0} \stackrel{a_{0}^{\prime \prime}}{\longrightarrow} A^{\prime}\right)
$$

with $M_{0} \in \mathrm{Ob} \mathcal{M}$. Then the induced functor

$$
\begin{aligned}
\mathcal{A} / \mathcal{M} & \stackrel{F}{\longrightarrow} \mathcal{B} / \mathcal{N} \\
\left(A \stackrel{a}{\longrightarrow} A^{\prime}\right) & \longmapsto\left(A F \stackrel{a F}{\longrightarrow} A^{\prime} F\right)
\end{aligned}
$$

is an equivalence.

Proof. We have to show that $\underline{F}$ is faithful. Suppose given $A \stackrel{a}{\longrightarrow} A^{\prime}$ in $\mathcal{A}$ such that

$$
\left(A F \stackrel{a F}{\longrightarrow} A^{\prime} F\right)=\left(A F \stackrel{b^{\prime}}{\longrightarrow} N \stackrel{b^{\prime \prime}}{\longrightarrow} A^{\prime} F\right),
$$

where $N \in \mathrm{Ob} \mathcal{N}$. Since $F$ is dense, we may assume $N=M F$ for some $M \in \mathrm{Ob} \mathcal{M}$. Since $F$ is full, there exist $a^{\prime}$ and $a^{\prime \prime}$ in $\mathcal{A}$ with $a^{\prime} F=b^{\prime}$ and $a^{\prime \prime} F=b^{\prime \prime}$. Then

$$
\left(A \stackrel{a}{\longrightarrow} A^{\prime}\right)=\left(A \stackrel{a^{\prime} a^{\prime \prime}}{\longrightarrow} A^{\prime}\right)+\left(A \stackrel{a_{0}}{\longrightarrow} A^{\prime}\right)
$$

with $a_{0} F=0$. Since $a^{\prime} a^{\prime \prime}$ factors over $M \in \mathrm{Ob} \mathcal{M}$, and since $a_{0}$ factors over an 
object of $\mathcal{M}$ by assumption on $F$, we conclude that $a$ factors over an object of $\mathcal{M}$.

\section{A.2. Exact categories}

\section{A.2.1 Definition}

The concept of exact categories is due to QuILLEN [27], who uses a different, but equivalent set of axioms. In [17, App. A], Keller has cut down redundancies in this set of axioms. We give still another equivalent reformulation.

An additive category $\mathcal{A}$ is a category with zero object, binary products and binary coproducts such that the natural map from the coproduct to the product is an isomorphism; which allows a commutative and associative addition $(+)$ to be defined on $\mathcal{A}(X, Y)$, where $X, Y \in \mathrm{Ob} \mathcal{A}$; and such that there exists an endomorphism $-1_{X}$ for each $X \in \mathrm{Ob} \mathcal{A}$ that is characterised by $1_{X}+\left(-1_{X}\right)=0_{X}$.

A sequence $X \stackrel{f}{\longrightarrow} Y \stackrel{g}{\longrightarrow} Z$ in $\mathcal{A}$ is called short exact if $f$ is a kernel of $g$ and $g$ is a cokernel of $f$.

A short exact sequence isomorphic to a short exact sequence of the form

$$
X \stackrel{(10)}{\longrightarrow} X \oplus Y \stackrel{\left(\begin{array}{l}
0 \\
1
\end{array}\right)}{\longrightarrow} Y,
$$

where $X, Y \in \mathrm{Ob} \mathcal{A}$, is called split short exact. A morphism appearing as a kernel in a split short exact sequence is split monomorphic, and a morphism appearing as a cokernel in a split short exact sequence is called split epimorphic. A split short exact sequence is isomorphic to a sequence of the form just displayed by an isomorphism having an identity on the first and on the third term.

An exact category $(\mathcal{E}, \mathcal{S})$ consists of an additive category $\mathcal{E}$ and an isomorphism closed set $\mathcal{S}$ of short exact sequences in $\mathcal{E}$, called pure short exact sequences, ${ }^{7}$ such that the following axioms (Ex 1, 2, 3, $\left.1^{\circ}, 2^{\circ}, 3^{\circ}\right)$ are satisfied.

A monomorphism fitting into a pure short exact sequence is called a pure monomorphism, denoted by $\rightarrow$; an epimorphism fitting into a pure short exact sequence is called a pure epimorphism, denoted by $\longrightarrow$. A morphism which can be written as a composition of a pure epimorphism followed by a pure monomorphism is called pure.

\footnotetext{
${ }^{7}$ This notion is borrowed from the particular cases of pure short exact sequences of lattices over orders and of $\otimes$-pure short exact sequences of modules. Other frequently used names are admissible short exact sequence, consisting of an admissible monomorphism and an admissible epimorphism; and conflation, consisting of an inflation and a deflation.
} 
Homology, Homotopy and Applications, vol. 9(2), 2007

(Ex 1) Split monomorphisms are pure monomorphisms.

$\left(\right.$ Ex $\left.1^{\circ}\right) \quad$ Split epimorphisms are pure epimorphisms.

(Ex 2) The composite of two pure monomorphisms is purely monomorphic.

(Ex $2^{\circ}$ ) The composite of two pure epimorphisms is purely epimorphic.

(Ex 3) Given a commutative diagram

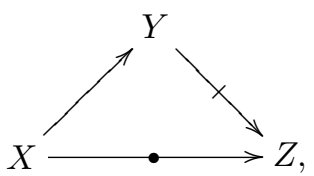

we may insert it into a commutative diagram

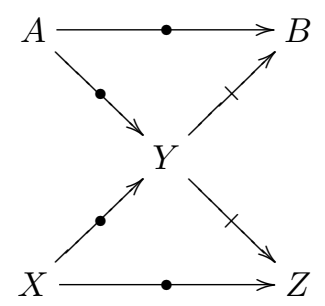

with $(X, Y, B)$ and $(A, Y, Z)$ pure short exact sequences.

$\left(\right.$ Ex $\left.3^{\circ}\right)$ Given a commutative diagram

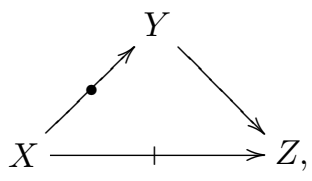

we may insert it into a commutative diagram

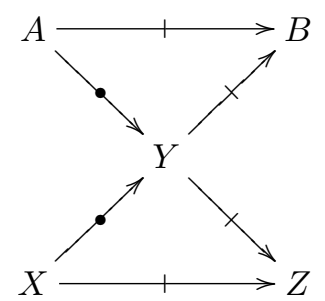

with $(X, Y, B)$ and $(A, Y, Z)$ pure short exact sequences.

An exact functor from an exact category $(\mathcal{E}, \mathcal{S})$ to an exact category $(\mathcal{F}, \mathcal{T})$ is given by an additive functor $\mathcal{E} \stackrel{F}{\longrightarrow} \mathcal{F}$ such that $\mathcal{S} F \subseteq \mathcal{T}$, where, by abuse of notation, $F$ also denotes the functor induced by $F$ on diagrams of shape $\longrightarrow \longrightarrow \longrightarrow$.

Frequently, the exact category $(\mathcal{E}, \mathcal{S})$ is simply referred to by $\mathcal{E}$.

Example A.2.

(1) An abelian category, equipped with the set of all short exact sequences as pure short exact sequences, is an exact category.

(2) If $\mathcal{E}$ is an exact category, so is $\mathcal{E}^{\circ}$, equipped with the pure short exact sequences 
of $\mathcal{E}$ considered as short exact sequences in $\mathcal{E}^{\circ}$, with the roles of kernel and cokernel interchanged.

Example A.3. An additive category $\mathcal{A}$, equipped with the set of split short exact sequences as pure short exact sequences, is an exact category.

In fact, (Ex 1,2) are fulfilled, and it remains to prove (Ex 3); then the dual axioms ensue by duality. Given

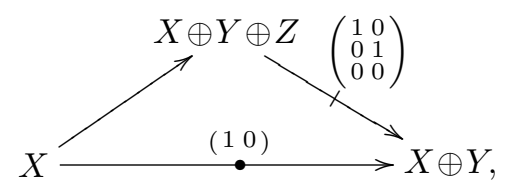

we get

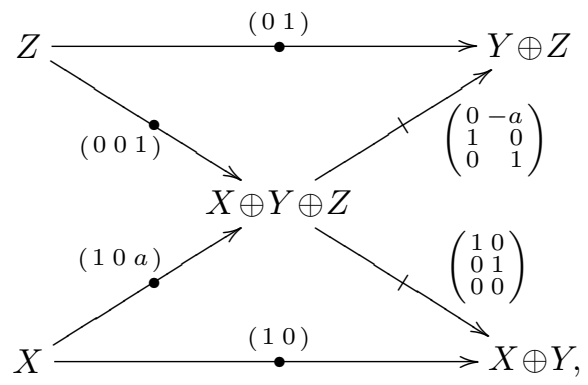

where $X \stackrel{a}{\longrightarrow} Z$ is the third component of the given morphism $X \longrightarrow X \oplus Y \oplus Z$.

Example A.4. Suppose given an exact category $\mathcal{E}$ and a category $D$. Let a short exact sequence $(X, Y, Z)$ in $\mathcal{E}(D)$ be pure if the sequence $\left(X_{d}, Y_{d}, Z_{d}\right)$ is a pure short exact sequence in $\mathcal{E}$ for all $d \in \mathrm{Ob} D$. Then $\mathcal{E}(D)$ is an exact category.

\section{A.2.2 EMbedding EXACT CATEgories}

By a theorem

- stated by Quillen [27, p. 100],

- proven by Laumon [21, Th. 1.0.3],

- re-proven by Keller [17, Prop. A.2],

- where Quillen resp. Keller refer to [9] for a similar resp. an auxiliary technique,

for any exact category $\mathcal{E}$, there exists an abelian category $\tilde{\mathcal{E}}$ containing $\mathcal{E}$ as a full subcategory closed under extensions, the pure short exact sequences in $\mathcal{E}$ being the short exact sequences in $\tilde{\mathcal{E}}$ with all three objects in $\mathrm{Ob} \mathcal{E}$.

Conversely, suppose given an exact category $\mathcal{E}$ and a full subcategory $\mathcal{E}^{\prime} \subseteq \mathcal{E}$ such that whenever $(X, Y, Z)$ is a pure short exact sequence in $\mathcal{E}$ with $X, Z \in \mathrm{Ob} \mathcal{E}^{\prime}$, then also $Y \in \operatorname{Ob} \mathcal{E}^{\prime}$. Then the subcategory $\mathcal{E}^{\prime}$, equipped with the pure short exact sequences in $\mathcal{E}$ with all three terms in $\mathrm{Ob} \mathcal{E}^{\prime}$ as pure short exact sequences in $\mathcal{E}^{\prime}$, is an exact category. 


\section{A.2.3 Frobenius Categories: Definitions}

\section{Definition A.5.}

(1) A bijective object in an exact category $\mathcal{E}$ is an object $B$ for which $\mathcal{E}(B,-)$ and $\mathcal{E}(-, B)$ are exact functors from $\mathcal{E}$ resp. from $\mathcal{E}^{\circ}$ to $\mathbf{Z}$-Mod.

(2) A Frobenius category is an exact category for which each object $X$ allows for a diagram $B \longrightarrow X \longrightarrow B^{\prime}$ with $B$ and $B^{\prime}$ bijective.

(3) Suppose given an exact category $\mathcal{F}$ carrying a shift automorphism $\mathrm{T}: X \longmapsto X \mathrm{~T}=X^{+1}$ and two additive endofunctors $\mathrm{I}$ and $\mathrm{P}$ together with natural transformations $1_{\mathcal{F}} \stackrel{\iota}{\longrightarrow} \mathrm{I}$ and $\mathrm{P} \stackrel{\pi}{\longrightarrow} 1_{\mathcal{F}}$ such that $\mathrm{TP}=\mathrm{I}$ and such that

$$
X \stackrel{X \iota}{\longrightarrow} X \mathrm{I}=X^{+1} \mathrm{P} \stackrel{X^{+1} \pi}{\longrightarrow} X^{+1}
$$

is a pure short exact sequence with bijective middle term for all $X \in \mathrm{Ob} \mathcal{C}$. Then $(\mathcal{F}, T, I, \iota, P, \pi)$ is called a functorial Frobenius category. Often we write just $\mathcal{F}$ for $(\mathcal{F}, \mathrm{T}, \mathrm{I}, \iota, \mathrm{P}, \pi)$.

Example A.6.

(1) Let $\mathcal{A}$ be an additive category. Let $\dot{\mathbf{Z}}$ denote the discrete category with $\mathrm{Ob} \dot{\mathbf{Z}}=$ $\mathbf{Z}$ and only identical morphisms. The category $\mathcal{A}(\dot{\mathbf{Z}})$ carries the shift functor $X^{\bullet} \longmapsto X^{\bullet+1}$, where $\left(X^{\bullet+1}\right)^{i}=X^{i+1}$. An object in the category $\mathrm{C}(\mathcal{A})$ of complexes with entries in $\mathcal{A}$ is written $\left(X^{\bullet}, d^{\bullet}\right)$, where $X$ is an object of $\mathcal{A}(\dot{\mathbf{Z}})$ and where $X^{\bullet} \stackrel{d^{\bullet}}{\longrightarrow} X^{\bullet+1}$ with $d^{\bullet} d^{\bullet+1}=0$. The category $\mathrm{C}(\mathcal{A})$, equipped with pointwise split short exact sequences, is an exact category; see Examples A.3, A.4. Given a complex $\left(X^{\bullet}, d^{\bullet}\right)$, we let $\left(X^{\bullet}, d^{\bullet}\right) \mathrm{T}=\left(X^{\bullet}, d^{\bullet}\right)^{+1}:=$ $\left(X^{\bullet+1},-d^{\bullet+1}\right)$ and

$$
\begin{aligned}
& \left(\left(X^{\bullet}, d^{\bullet}\right) \stackrel{\left(X^{\bullet}, d^{\bullet}\right) \iota}{\longrightarrow}\left(X^{\bullet}, d^{\bullet}\right) \mathbf{I}=\left(X^{\bullet}, d^{\bullet}\right)^{+1} \mathrm{P} \stackrel{\left(X^{\bullet}, d^{\bullet}\right)^{+1} \pi}{\longrightarrow}\left(X^{\bullet}, d^{\bullet}\right)^{+1}\right) \\
:= & \left(\left(X^{\bullet}, d^{\bullet}\right) \stackrel{\left(1 d^{\bullet}\right)}{\longrightarrow}\left(X^{\bullet} \oplus X^{\bullet+1},\left(\begin{array}{ll}
0 & 0 \\
1 & 0
\end{array}\right)\right) \stackrel{\left(\begin{array}{c}
-d^{\bullet} \\
1
\end{array}\right)}{\longrightarrow}\left(X^{\bullet+1},-d^{\bullet+1}\right)\right) .
\end{aligned}
$$

Then $(\mathrm{C}(\mathcal{A}), \mathrm{T}, \mathrm{I}, \iota, \mathrm{P}, \pi)$ is a functorial Frobenius category.

(2) Suppose $\mathcal{E}$ to be a Frobenius category. Let $\mathcal{B} \subseteq \mathcal{E}$ be a sufficiently large full subcategory of bijective objects, i.e. each object of $\mathcal{B}$ is bijective in $\mathcal{E}$, and each object $X$ of $\mathcal{E}$ admits $B \longrightarrow X \rightarrow B^{\prime}$ with $B, B^{\prime} \in \mathrm{Ob} \mathcal{B}$. In other words, each bijective object of $\mathcal{E}$ is isomorphic to a direct summand of an object of $\mathcal{B}$.

Let $\mathcal{B}^{\text {ac }} \subseteq \mathrm{C}(\mathcal{B})$ denote the full subcategory of purely acyclic complexes, i.e. complexes $\left(X^{\bullet}, d^{\bullet}\right)$ such that all differentials $X^{i} \stackrel{d}{\longrightarrow} X^{i+1}$ are pure, factoring as $d=\bar{d} \dot{d}$ with $\bar{d}$ purely epi- and $\dot{d}$ purely monomorphic, and such that all resulting sequences $(\dot{d}, \bar{d})$ are purely short exact. For short, a complex is purely acyclic if it decomposes into pure short exact sequences.

Then $\mathcal{B}^{\text {ac }}$ is a functorial Frobenius category, equipped with the restricted functors and transformations of $\mathrm{C}(\mathcal{B})$ as defined in (1); cf. [23, Lem. 1.1]. Let $\mathcal{B}^{\text {sp ac }} \subseteq \mathcal{B}^{\text {ac }}$ be the full subcategory of split acyclic complexes, i.e. of complexes isomorphic to a complex of the form $\left(T^{\bullet} \oplus T^{\bullet+1},\left(\begin{array}{ll}0 & 0 \\ 1 & 0\end{array}\right)\right)$ for some 
$T^{\bullet} \in \operatorname{Ob} \mathcal{B}(\dot{\mathbf{Z}})$. Then $\mathcal{B}^{\text {sp ac }}$ is a sufficiently large full subcategory of bijective objects in $\mathcal{B}^{\text {ac }}$.

Definition A.7. Suppose given a Frobenius category $\mathcal{E}$, and a sufficiently large full subcategory $\mathcal{B} \subseteq \mathcal{E}$ of bijectives. Let

$$
\begin{array}{ll}
\mathcal{E} & :=\mathcal{E} / \mathcal{B} \quad \text { be the classical stable category of } \mathcal{E} ; \text { and } \\
\underline{\underline{\mathcal{E}}}:=\mathcal{B}^{\text {ac }} / \mathcal{B}^{\text {sp ac }} \text { be the stable category of } \mathcal{E} . &
\end{array}
$$

In other words, the stable category $\underline{\underline{\mathcal{E}}}$ of $\mathcal{E}$ is defined to be the classical stable category $\underline{\mathcal{B}^{\text {ac }}}$ of $\mathcal{B}^{\text {ac }}$. The shift functor induced by the automorphism $\mathrm{T}$ of $\mathcal{B}^{\text {ac }}$ on $\underline{\underline{\mathcal{E}}}$ is also denoted by $\mathrm{T}$.

Lemma A.8. The functor

$$
\begin{aligned}
\mathcal{B}^{\mathrm{ac}} \stackrel{I}{\longrightarrow} \mathcal{E} \\
(X, d) \longmapsto \operatorname{Im}\left(X^{0} \stackrel{d}{\longrightarrow} X^{1}\right)
\end{aligned}
$$

induces an equivalence

$$
\underline{\underline{\mathcal{E}}}=\underline{\mathcal{B}^{\mathrm{ac}}} \stackrel{\underline{I}}{\sim} \underline{\mathcal{E}} .
$$

Proof. This is an application of Lemma A.1. Cf. [19, Sec. 4.3].

We choose an inverse equivalence $R$ to $\underline{I}$. We have the residue class functor $\mathcal{E} \stackrel{N}{\longrightarrow} \underline{\mathcal{E}}$, and, by abuse of notation, a second residue class functor $(\mathcal{E} \stackrel{N}{\longrightarrow} \underline{\underline{\mathcal{E}}}):=$ $(\mathcal{E} \stackrel{N}{\longrightarrow} \underline{\mathcal{E}} \stackrel{R}{\sim} \mathcal{E})$.

Finally, we mention that a morphism $X \stackrel{f}{\longrightarrow} Y$ is zero in $\underline{\mathcal{E}}$ if and only if for any monomorphism $X \stackrel{i}{\rightarrow} X^{\prime}$ and any epimorphism $Y^{\prime} \stackrel{p}{\longrightarrow} Y$, there is a factorisation $f=i f^{\prime} p$. This defines $\mathcal{E}$ without mentioning bijective objects in $\mathcal{E}$. So one might speculate whether the class of Frobenius categories within the class of exact categories could be extended without losing essential properties of Frobenius categories.

\section{A.3. Kernel-cokernel-criteria}

Let $\mathcal{A}$ be an abelian category. The circumference lemma states that given a commutative triangle in $\mathcal{A}$, the induced sequence on kernels and cokernels, with zeroes attached to the ends, is long exact.

Definition A.9. A weak square in $\mathcal{A}$ is a commutative quadrangle $(A, B, C, D)$ in $\mathcal{A}$ whose diagonal sequence $(A, B \oplus C, D)$ is exact at $B \oplus C$. It is denoted by a "+"-sign in the commutative diagram,

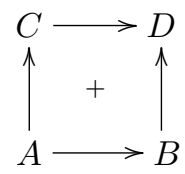

A pullback is a weak square with first morphism in the diagonal sequence being 
monomorphic. It is denoted

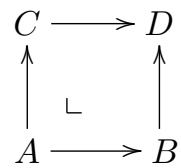

A pushout is a weak square with second morphism in the diagonal sequence being epimorphic. It is denoted

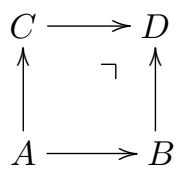

A square is a commutative quadrangle that is a pullback and a pushout, i.e. that has a short exact diagonal sequence. It is denoted

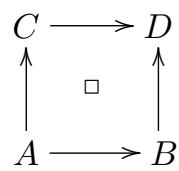

Remark $A$.10. If a commutative quadrangle in $\mathcal{A}$

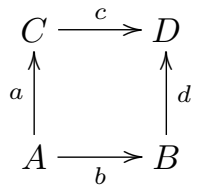

is a square, then the induced morphism from the kernel of $A \stackrel{a}{\longrightarrow} C$ to the kernel of $B \stackrel{d}{\longrightarrow} D$ is an isomorphism and the induced morphism from the cokernel of $A \stackrel{a}{\longrightarrow} C$ to the cokernel of $B \stackrel{d}{\longrightarrow} D$ is an isomorphism.

Proof. If $(A, B, C, D)$ is a square, then the circumference lemma, applied to the commutative triangle

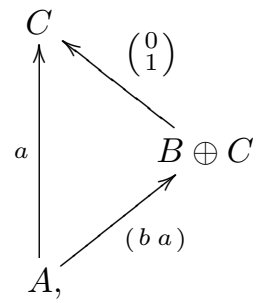

yields a long exact sequence

$$
0 \longrightarrow \mathrm{K}_{a} \stackrel{j}{\longrightarrow} B \stackrel{-d}{\longrightarrow} D \stackrel{q}{\longrightarrow} \mathrm{C}_{a} \longrightarrow 0
$$

where $\mathrm{K}_{a} \stackrel{i}{\longrightarrow} A$ is the kernel of $a$, and where $C \stackrel{p}{\longrightarrow} \mathrm{C}_{a}$ is the cokernel of $a$. Since $i b=j$ and $c b=p$, the induced morphisms on the kernels and on the cokernels of $a$ 
and $d$ are isomorphisms.

Lemma A.11. A commutative quadrangle in $\mathcal{A}$

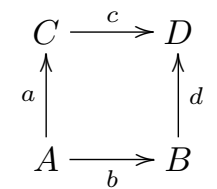

is a weak square if and only if the induced morphism $\mathrm{K}_{a} \longrightarrow \mathrm{K}_{d}$ from the kernel of $A \stackrel{a}{\longrightarrow} C$ to the kernel of $B \stackrel{d}{\longrightarrow} D$ is an epimorphism and the induced morphism $\mathrm{C}_{a} \longrightarrow \mathrm{C}_{d}$ from the cokernel of $A \stackrel{a}{\longrightarrow} C$ to the cokernel of $B \stackrel{d}{\longrightarrow} D$ is a monomorphism.

It is a pullback if and only if $\mathrm{K}_{a} \longrightarrow \mathrm{K}_{d}$ and $\mathrm{C}_{a} \rightarrow \mathrm{C}_{d}$.

It is a pushout if and only if $\mathrm{K}_{a} \longrightarrow \mathrm{K}_{d}$ and $\mathrm{C}_{a} \stackrel{\sim}{\longrightarrow} \mathrm{C}_{d}$.

It is a square if and only if $\mathrm{K}_{a} \stackrel{\sim}{\longrightarrow} \mathrm{K}_{d}$ and $\mathrm{C}_{a} \stackrel{\sim}{\longrightarrow} \mathrm{C}_{d}$.

Proof. Let $A^{\prime}$ be the pullback of $(C, B, D)$, and let $D^{\prime}$ be the pushout of $\left(A^{\prime}, C, B\right)$. We obtain induced morphisms $A \longrightarrow A^{\prime}$ and $D^{\prime} \longrightarrow D$. The circumference lemma, applied to $\left(B, D^{\prime}, D\right)$, shows $\mathrm{C}_{B \rightarrow D^{\prime}} \rightarrow \mathrm{C}_{B \rightarrow D}$.

The quadrangle $(A, B, C, D)$ is a weak square if and only if $A \longrightarrow A^{\prime}$, which in turn, by the circumference lemma applied to $\left(A, A^{\prime}, C\right)$, is equivalent to $\mathrm{K}_{A \rightarrow C} \mapsto \mathrm{K}_{A^{\prime} \rightarrow C}$ and $\mathrm{C}_{A \rightarrow C} \stackrel{\sim}{\longrightarrow} \mathrm{C}_{A^{\prime} \rightarrow C}$, which, by composition and by Remark A.10, applied to the square $\left(A^{\prime}, B, C, D^{\prime}\right)$, is equivalent to $\mathrm{K}_{A \rightarrow C} \longrightarrow \mathrm{K}_{B \rightarrow D}$ and $\mathrm{C}_{A \rightarrow C} \rightarrow \mathrm{C}_{B \rightarrow D}$.

The quadrangle $(A, B, C, D)$ is a pullback if and only if $A \stackrel{\sim}{\longrightarrow}$, which in turn, by the circumference lemma applied to $\left(A, A^{\prime}, C\right)$, is equivalent to $\mathrm{K}_{A \rightarrow C} \stackrel{\sim}{\longrightarrow}$ $\mathrm{K}_{A^{\prime} \rightarrow C}$ and $\mathrm{C}_{A \rightarrow C} \stackrel{\sim}{\longrightarrow} \mathrm{C}_{A^{\prime} \rightarrow C}$, which, by composition and by Remark A.10, applied to the square $\left(A^{\prime}, B, C, D^{\prime}\right)$, is equivalent to $\mathrm{K}_{A \rightarrow C} \stackrel{\sim}{\longrightarrow} \mathrm{K}_{B \rightarrow D}$ and $\mathrm{C}_{A \rightarrow C} \rightarrow$ $\mathrm{C}_{B \rightarrow D}$.

The quadrangle $(A, B, C, D)$ is a square if and only if $A \stackrel{\sim}{\longrightarrow} A^{\prime}$ and $D^{\prime} \stackrel{\sim}{\longrightarrow} D$, which in turn, by the circumference lemma applied to $\left(A, A^{\prime}, C\right)$, is equivalent to $\mathrm{K}_{A \rightarrow C} \stackrel{\sim}{\longrightarrow} \mathrm{K}_{A^{\prime} \rightarrow C}, \mathrm{C}_{A \rightarrow C} \stackrel{\sim}{\longrightarrow} \mathrm{C}_{A^{\prime} \rightarrow C}$ and $\mathrm{C}_{B \rightarrow D^{\prime}} \stackrel{\sim}{\longrightarrow} \mathrm{C}_{B \rightarrow D}$, which, by composition and by Remark A.10, applied to the square $\left(A^{\prime}, B, C, D^{\prime}\right)$, is equivalent to $\mathrm{K}_{A \rightarrow C} \stackrel{\sim}{\longrightarrow} \mathrm{K}_{B \rightarrow D}$ and $\mathrm{C}_{A \rightarrow C} \stackrel{\sim}{\longrightarrow} \mathrm{C}_{B \rightarrow D}$.

\section{A.4. An exact lemma}

Let $\mathcal{E}$ be an exact category. A pure square in $\mathcal{E}$ is a commutative quadrangle $(A, B, C, D)$ in $\mathcal{E}$ that has a pure short exact diagonal sequence $(A, B \oplus C, D)$. Like a square in abelian categories, a pure square is denoted by a box " $\square$ ".

Lemma A.12. Suppose given a composition

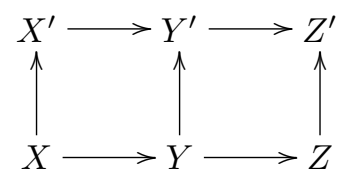

of commutative quadrangles in $\mathcal{E}$. If two out of the three quadrangles $\left(X, Y, X^{\prime}, Y^{\prime}\right)$, 
$\left(Y, Z, Y^{\prime}, Z^{\prime}\right),\left(X, Z, X^{\prime}, Z^{\prime}\right)$ are pure squares, so is the third.

Proof. In an abelian category, this follows from Lemma A.11.

As explained in $\S$ A.2.2, we may embed $\mathcal{E}$ fully, faithfully and additively into an abelian category $\tilde{\mathcal{E}}$ such that the pure short exact sequences in $\mathcal{E}$ are precisely the short exact sequences in $\tilde{\mathcal{E}}$ with all three objects in $\mathrm{Ob} \mathcal{E}$. In particular, the pure squares in $\mathcal{E}$ are precisely the squares in $\tilde{\mathcal{E}}$ with all four objects in $\mathrm{Ob} \mathcal{E}$, and the assertion in $\mathcal{E}$ follows from the assertion in $\tilde{\mathcal{E}}$.

\section{A.5. Some abelian lemmata}

Let $\mathcal{A}$ be an abelian category.

Lemma A.13. Inserting images, a weak square $(A, B, C, D)$ in $\mathcal{A}$ decomposes into

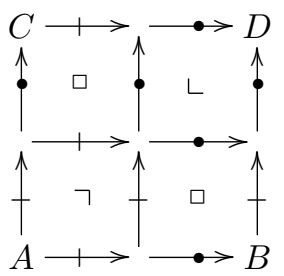

Proof. The assertion follows using the characterisation of weak squares, pullbacks and pushouts given in Lemma A.11.

Lemma A.14. If, in a commutative diagram

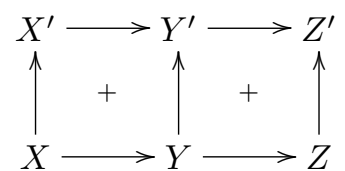

in $\mathcal{A}$, the quadrangles $\left(X, Y, X^{\prime}, Y^{\prime}\right)$ and $\left(Y, Z, Y^{\prime}, Z^{\prime}\right)$ are weak squares, then the composite quadrangle $\left(X, Z, X^{\prime}, Z^{\prime}\right)$ is also a weak square.

Proof. The assertion follows using the characterisation of weak squares given in Lemma A.11.

Lemma A.15. If, in a commutative diagram

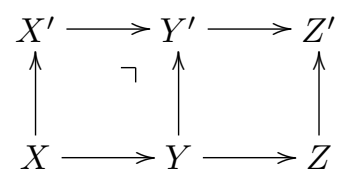

in $\mathcal{A}$, the left hand side quadrangle $\left(X, Y, X^{\prime}, Y^{\prime}\right)$ is a pushout, as indicated, and the outer quadrangle $\left(X, Z, X^{\prime}, Z^{\prime}\right)$ is a weak square, then the right hand side quadrangle $\left(Y, Z, Y^{\prime}, Z^{\prime}\right)$ is also a weak square.

If the left hand side quadrangle $\left(X, Y, X^{\prime}, Y^{\prime}\right)$ and the outer quadrangle $\left(X, Z, X^{\prime}\right.$, $\left.Z^{\prime}\right)$ are pushouts, then the right hand side quadrangle $\left(Y, Z, Y^{\prime}, Z^{\prime}\right)$ is also a pushout. 
Proof. This follows using Lemma A.11.

Lemma A.16. If, in a commutative quadrangle in $\mathcal{A}$

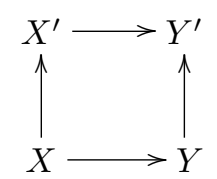

the morphism $X \longrightarrow Y$ is an epimorphism and the morphism $X^{\prime} \longrightarrow Y^{\prime}$ is a monomorphism, then the quadrangle is a weak square.

Proof. This follows using Lemma A.11, applied horizontally.

Lemma A.17. Given a commutative diagram

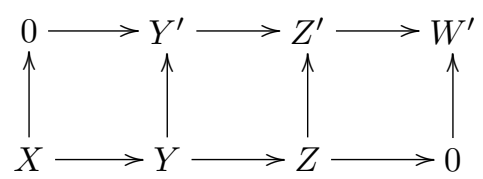

in $\mathcal{A}$ such that $\left(X, Z, 0, Z^{\prime}\right)$ and $\left(Y, 0, Y^{\prime}, W^{\prime}\right)$ are weak squares, then $\left(Y, Z, Y^{\prime}, Z^{\prime}\right)$ is a weak square.

Proof. This follows using Lemma A.11.

Lemma A.18. Given a diagram

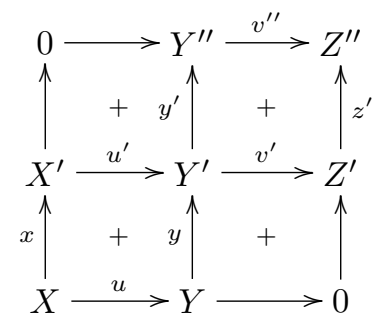

in $\mathcal{A}$ consisting of weak squares, as indicated by + , the sequence

$$
X \stackrel{x u^{\prime}}{\longrightarrow} Y^{\prime} \stackrel{\left(y^{\prime} v^{\prime}\right)}{\longrightarrow} Y^{\prime \prime} \oplus Z^{\prime} \stackrel{\left(\begin{array}{c}
v^{\prime \prime} \\
-z^{\prime}
\end{array}\right)}{\longrightarrow} Z^{\prime \prime}
$$

is exact at $Y^{\prime}$ and at $Y^{\prime \prime} \oplus Z^{\prime}$.

Proof. At $Y^{\prime}$, we reduce to the case $u, u^{\prime}, x$ and $y$ monomorphic and $\left(X, Y, X^{\prime}, Y^{\prime}\right)$ being a pullback via Lemma A.13. Suppose given $T \stackrel{t}{\longrightarrow} Y^{\prime}$ with $t y^{\prime}=0$ and $t v^{\prime}=0$. First of all, there exist $T \stackrel{a}{\longrightarrow} X^{\prime}$ and $T \stackrel{b}{\longrightarrow} Y$ such that $a u^{\prime}=t=b y$. Thus there exists $T \stackrel{c}{\longrightarrow} X$ such that $c x=a$ and $c u=b$. In particular, $c x u^{\prime}=a u^{\prime}=t$. Hence a factorisation of $t$ over $x u^{\prime}$ exists. Uniqueness follows by monomorphy of $x u^{\prime}$.

Lemma A.19. The following hold. 
(1) Suppose given a weak square in $\mathcal{A}$

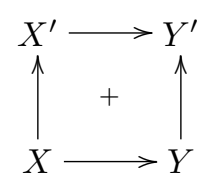

with $X^{\prime}$ bijective. If the images of $X \longrightarrow Y$, of $X \longrightarrow X^{\prime}$ and of $Y \longrightarrow Y^{\prime}$ are bijective, then the images of $X^{\prime} \longrightarrow Y^{\prime}$ and of $X \longrightarrow Y^{\prime}$ are bijective, too.

(2) Suppose given a weak square in $\mathcal{A}$

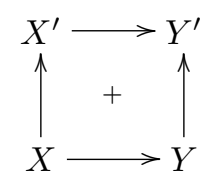

with $Y$ bijective. If the images of $X^{\prime} \longrightarrow Y^{\prime}$, of $X \longrightarrow X^{\prime}$ and of $Y \longrightarrow Y^{\prime}$ are bijective, then the images of $X \longrightarrow Y$ and of $X \longrightarrow Y^{\prime}$ are bijective, too.

Proof. Ad (1). We decompose $\left(X, Y, X^{\prime}, Y^{\prime}\right)$ according to Lemma A.13 and denote the image of $X \longrightarrow Y$ by $\operatorname{Im}_{X, Y}$, etc.

The diagonal sequence of the square $\left(\operatorname{Im}_{X, Y}, Y, \operatorname{Im}_{X, Y^{\prime}}, \operatorname{Im}_{Y, Y^{\prime}}\right)$ shows that $\operatorname{Im}_{X, Y^{\prime}}$ is bijective.

The diagonal sequence of the square $\left(\operatorname{Im}_{X, X^{\prime}}, \operatorname{Im}_{X, Y^{\prime}}, X^{\prime}, \operatorname{Im}_{X^{\prime}, Y^{\prime}}\right)$ shows that $\operatorname{Im}_{X^{\prime}, Y^{\prime}}$ is bijective.

Lemma A.20. Given a pullback

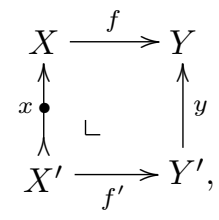

in $\mathcal{A}$ with $Y^{\prime}$ injective, the morphism $\left(X^{\prime}, Y^{\prime}\right) \stackrel{(x, y)}{\longrightarrow}(X, Y)$ is split monomorphic in $\mathcal{A}\left(\Delta_{1}\right)$. More precisely, any retraction for $x$ may be extended to a retraction for $(x, y)$.

Proof. Let $x x^{\prime}=1_{X^{\prime}}$. We form the pushout.

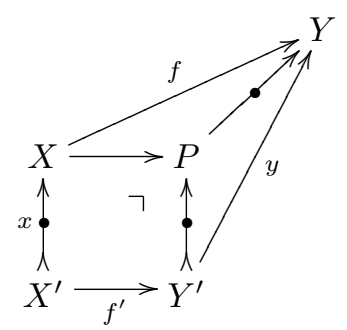

There is an induced morphism $P \longrightarrow Y^{\prime}$ such that $\left(X \longrightarrow P \longrightarrow Y^{\prime}\right)=$ 
$\left(X \stackrel{x^{\prime} f^{\prime}}{\longrightarrow} Y^{\prime}\right)$ and such that $\left(Y^{\prime} \longrightarrow P \longrightarrow Y^{\prime}\right)=\left(Y^{\prime} \stackrel{1_{Y^{\prime}}}{\longrightarrow} Y^{\prime}\right)$. Since $Y^{\prime}$ is injective, we obtain a factorisation $\left(P \longrightarrow Y^{\prime}\right)=\left(P \longrightarrow Y \longrightarrow Y^{\prime}\right)$.

Lemma A.21. Suppose given a morphism $X \longrightarrow Y$ of commutative quadrangles in $\mathcal{A}$, i.e. a morphism in $\mathcal{A}\left(\Delta_{1} \times \Delta_{1}\right)$.

(1) If $X$ is a pushout and $Y$ is a weak square, then the cokernel of $X \longrightarrow Y$ is a weak square.

(2) If $X$ is a weak square and $Y$ is a pullback, then the kernel of $X \longrightarrow Y$ is a weak square.

Proof. Ad (1). A morphism of commutative quadrangles gives rise to a morphism of the diagonal sequences; namely from a sequence that is exact in the middle and has an epimorphic second morphism, stemming from $X$, to a sequence that is exact in the middle, stemming from $Y$. In order to prove that the cokernel sequence is exact in the middle, we reduce by insertion of the image of the first morphism of the diagonal sequence and by an application of the circumference lemma to the case in which the sequence stemming from $Y$ has a monomorphic first morphism. Then the snake lemma yields the result.

Lemma A.22. Suppose given a diagram

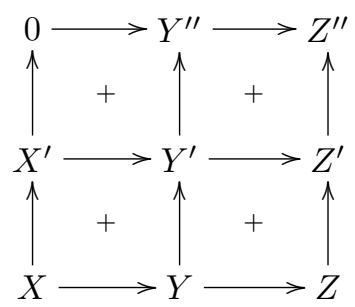

in $\mathcal{A}$, consisting of weak squares. The induced morphisms furnish a short exact sequence

$$
\operatorname{Im}\left(X \longrightarrow Z^{\prime}\right) \longrightarrow \operatorname{Im}\left(Y \longrightarrow Z^{\prime}\right) \longrightarrow \operatorname{Im}\left(Y \longrightarrow Z^{\prime \prime}\right) .
$$

Proof. Abbreviate $\operatorname{Im}\left(X \longrightarrow Z^{\prime}\right)$ by $\operatorname{Im}_{X, Z^{\prime}}$ etc. The morphism $\operatorname{Im}_{X, Z^{\prime}} \longrightarrow \operatorname{Im}_{Y, Z^{\prime}}$ is monomorphic by composition, and, dually, the morphism $\operatorname{Im}_{Y, Z^{\prime}} \longrightarrow \operatorname{Im}_{Y, Z^{\prime \prime}}$ is epimorphic. Now since $\operatorname{Im}_{X, X^{\prime}} \longrightarrow \operatorname{Im}_{X, Z^{\prime}}$ is epimorphic and $\operatorname{Im}_{Y, Z^{\prime \prime}} \longrightarrow \operatorname{Im}_{Y^{\prime \prime}, Z^{\prime \prime}}$ is monomorphic, it suffices to show that

$$
\operatorname{Im}_{X, X^{\prime}} \longrightarrow \operatorname{Im}_{Y, Z^{\prime}} \longrightarrow \operatorname{Im}_{Y^{\prime \prime}, Z^{\prime \prime}}
$$

is exact at $\operatorname{Im}_{Y, Z^{\prime}}$. This follows from the diagram obtained by Lemma A.13

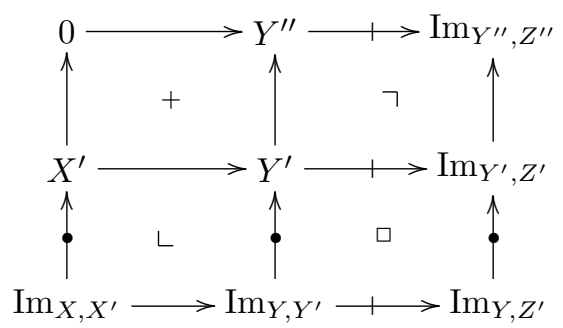


since by Lemma A.14, weak squares are stable under composition.

\section{A.6. On Frobenius categories}

A.6.1 Some Frobenius-abelian lemmata

Suppose given an abelian Frobenius category $\mathcal{A}$; see Definition A.5. Let $\mathcal{B}$ be its full subcategory of bijective objects. Recall that the classical stable category of $\mathcal{A}$ is defined as $\underline{\mathcal{A}}=\mathcal{A} / \mathcal{B}$; see Definition A.7. A morphism in $\mathcal{A}$ whose residue class in $\mathcal{A}$ is an isomorphism is called a homotopism. A morphism in $\mathcal{A}$ whose residue class in $\underline{\mathcal{A}}$ is a retraction is called a retraction up to homotopy.

Lemma A.23. Given a retraction up to homotopy $X \stackrel{f}{\longrightarrow} Y$ and an epimorphism $Y^{\prime} \stackrel{y}{\longrightarrow} Y$ in $\mathcal{A}$, in the pullback

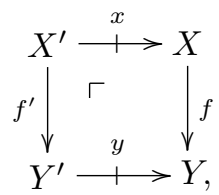

the morphism $X^{\prime} \stackrel{f^{\prime}}{\longrightarrow} Y^{\prime}$ is a retraction up to homotopy, too. More precisely, if $g f \equiv_{\mathcal{B}} 1_{Y}$, then we may find a morphism $g^{\prime}$ with $g^{\prime} f^{\prime} \equiv_{\mathcal{B}} 1_{Y^{\prime}}$ as a pullback of $g$ along $x$.

Proof. Let $Y \stackrel{g}{\longrightarrow} X$ be such that $g f=1_{Y}+h$, where

$$
(Y \stackrel{h}{\longrightarrow} Y)=\left(Y \stackrel{h_{1}}{\longrightarrow} B \stackrel{h_{2}}{\longrightarrow} Y\right)
$$

for some $B \in \mathrm{Ob} \mathcal{B}$ and some morphisms $h_{1}$ and $h_{2}$ in $\mathcal{A}$. Let $B \stackrel{h_{2}^{\prime}}{\longrightarrow} Y^{\prime}$ be a morphism such that

$$
\left(B \stackrel{h_{2}^{\prime}}{\longrightarrow} Y^{\prime} \stackrel{y}{\longrightarrow} Y\right)=\left(B \stackrel{h_{2}}{\longrightarrow} Y\right),
$$

which exists since $B$ is projective and $y$ is epimorphic. The commutative quadrangle

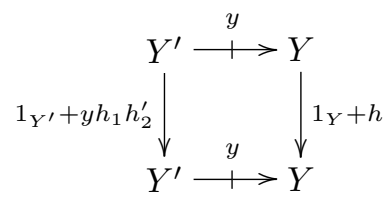

is a pullback since the induced morphism on the horizontal kernels is an identity; see Lemma A.11. So we may form the diagram

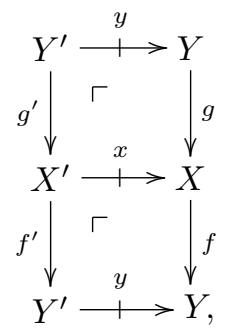


in which $g^{\prime}$ with $g^{\prime} x=y g$ and $g^{\prime} f^{\prime}=1_{Y^{\prime}}+y h_{1} h_{2}^{\prime}$ is induced by the universal property of the lower pullback $\left(X^{\prime}, X, Y^{\prime}, Y\right)$, and in which the resulting upper quadrangle $\left(Y^{\prime}, Y, X^{\prime}, X\right)$ is a pullback by Lemma A.11.

Lemma A.24. Given a homotopism $X \stackrel{f}{\longrightarrow} Y$ and an epimorphism $Y^{\prime} \stackrel{y}{\longrightarrow} Y$ in $\mathcal{A}$, in the pullback

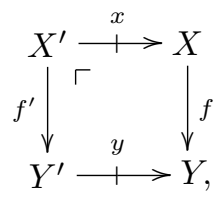

the morphism $X^{\prime} \stackrel{f^{\prime}}{\longrightarrow} Y^{\prime}$ is a homotopism, too.

Proof. Let $g f \equiv_{\mathcal{B}} 1_{Y}$ and $f g \equiv_{\mathcal{B}} 1_{X}$. By Lemma A.23, we may form the diagram

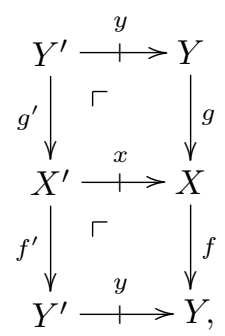

in which $g^{\prime} f^{\prime} \equiv_{\mathcal{B}} 1_{Y^{\prime}}$. Since $g$ is a retraction up to homotopy, so is $g^{\prime}$ by Lemma A.23. Therefore $g^{\prime}$ is a homotopism. Hence also $f^{\prime}$ is a homotopism.

\section{A.6.2 Decomposing SPlit Diagrams in Intervals}

Let $\mathcal{A}$ be an abelian Frobenius category, and let $\mathcal{B}$ be its full subcategory of bijective objects. Suppose given $n \geqslant 1$. Write $\dot{\Delta}_{n}:=\Delta_{n} \backslash\{0\}$. An object $X$ in $\mathcal{A}\left(\dot{\Delta}_{n}\right)$ is called split if $X_{k} \stackrel{x}{\longrightarrow} X_{l}$ is split for all $k, l \in[1, n]$ with $k \leqslant l$.

Given $C \in \operatorname{Ob} \mathcal{A}$ and $k, l \in[1, n]$ with $k \leqslant l$, we denote by $C_{[k, l]}$ the object of $\mathcal{A}\left(\dot{\Delta}_{n}\right)$ given by $\left(C_{[k, l]}\right)_{j}=0$ for $j \in[1, n] \backslash[k, l]$, by $\left(C_{[k, l]}\right)_{j}=C$ for $j \in[k, l]$, and by $\left(\left(C_{[k, l]}\right)_{j} \stackrel{c}{\longrightarrow}\left(C_{[k, l]}\right)_{j^{\prime}}\right)=\left(C \stackrel{1_{C}}{\longrightarrow} C\right)$ for $j, j^{\prime} \in[k, l]$ with $j \leqslant j^{\prime}$. An object in $\mathcal{A}\left(\dot{\Delta}_{n}\right)$ of the form $C_{[k, l]}$ for some $C \in \mathrm{Ob} \mathcal{A}$ and some $k, l \in[1, n]$ with $k \leqslant l$ is called an interval.

Lemma A.25. Any split object in $\mathcal{B}\left(\dot{\Delta}_{n}\right)$ is isomorphic to a finite direct sum of intervals.

Proof. We proceed by induction on $n$. Suppose given a split object $X$ in $\mathcal{B}\left(\dot{\Delta}_{n}\right)$. Let $X^{\prime}:=X \uparrow_{0}$ be defined as a pointwise pullback at $n$, using $0 \stackrel{0}{\longrightarrow} X_{n}$ (see $\S$ A.7 below). We have $X^{\prime} \in \operatorname{Ob} \mathcal{B}\left(\dot{\Delta}_{n}\right)$ with $X_{n}^{\prime}=0$. Hence, by induction, $X^{\prime}$ is isomorphic to a finite direct sum of intervals. There is a pure monomorphism $X^{\prime} \rightarrow X$ whose cokernel is a diagram in $\mathrm{Ob} \mathcal{B}\left(\dot{\Delta}_{n}\right)$ consisting of split monomorphisms; see Lemma A.11. Moreover, by an iterated application of Lemma A.20, starting at 
position 1 , this pure monomorphism $X^{\prime} \rightarrow X$ is split as a morphism ${ }^{8}$ of $\mathcal{A}\left(\dot{\Delta}_{n}\right)$. Thus $X$ is isomorphic to the direct sum of $X^{\prime}$ and the cokernel of $X^{\prime} \rightarrow X$, and it remains to be shown that this cokernel is isomorphic to a finite direct sum of intervals.

Therefore, we may assume that $X$ consists of split monomorphisms $X_{k}>\stackrel{x}{\bullet}>X_{l}$ for $k, l \in[1, n]$. We have a monomorphism $\left(X_{1}\right)_{[1, n]} \stackrel{i}{\rightarrow} X$. Choosing a retraction to $X_{1}>\stackrel{x}{\bullet} X_{n}$ and composing, we obtain a coretraction to $i$, so that $X$ is isomorphic to the direct sum of the interval $\left(X_{1}\right)_{[1, n]}$ and the cokernel of $i$. Since the cokernel of $i$ has a zero term at position 1 , we are done, by induction.

\section{A.6.3 A Freyd CATEGORY REMindeR}

The construction of the Freyd category and its properties are due to FREYD [8, Th. 3.1].

Definition A.26. Suppose given an additive category $\mathcal{C}$ and a morphism $X \stackrel{f}{\longrightarrow} Y$ in $\mathcal{C}$.

(1) A morphism $K \stackrel{i}{\longrightarrow} X$ is a weak kernel of $X \stackrel{f}{\longrightarrow} Y$ if the sequence of abelian groups

$$
(T, K) \stackrel{(-) i}{\longrightarrow}(T, X) \stackrel{(-) f}{\longrightarrow}(T, Y)
$$

is exact at $(T, X)$ for every $T \in \mathrm{Ob} \mathcal{C}$.

(2) A morphism $Y \stackrel{p}{\longrightarrow} C$ is a weak cokernel of $X \stackrel{f}{\longrightarrow} Y$ if the sequence of abelian groups

$$
(X, T) \stackrel{f(-)}{\longleftarrow}(Y, T) \stackrel{p(-)}{\longleftarrow}(C, T)
$$

is exact at $(Y, T)$ for every $T \in \mathrm{Ob} \mathcal{C}$.

(3) The category $\mathcal{C}$ is called weakly abelian if every morphism has a weak kernel and a weak cokernel, and if every morphism is a weak kernel (of some morphism) and a weak cokernel (of some morphism).

Let $\mathcal{C}$ be a weakly abelian category. Let $\mathcal{C}^{0}\left(\Delta_{1}\right)$ be the full subcategory of $\mathcal{C}\left(\Delta_{1}\right)$ whose objects are zero morphisms. The Freyd category $\hat{\mathcal{C}}$ of $\mathcal{C}$ is defined to be the quotient category

$$
\hat{\mathcal{C}}:=\mathcal{C}\left(\Delta_{1}\right) / \mathcal{C}^{0}\left(\Delta_{1}\right)
$$

We collect some elementary facts and constructions and mention some conventions.

(1) The category $\hat{\mathcal{C}}$ is abelian. The kernel and the cokernel of a morphism $X \stackrel{f}{\longrightarrow} Y$

\footnotetext{
${ }^{8}$ At this point, we use that $\dot{\Delta}_{n}$ is linearly ordered.
} 
Homology, Homotopy and Applications, vol. 9(2), 2007

represented by $\left(f^{\prime}, f^{\prime \prime}\right)$ are constructed as

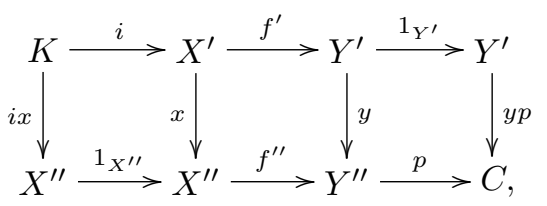

where $i$ is a chosen weak kernel and $p$ a chosen weak cokernel of the diagonal morphism $f^{\prime} y=x f^{\prime \prime}$. If $f^{\prime} y=x f^{\prime \prime}=0$, we choose $X^{\prime} \stackrel{1_{X^{\prime}}}{\longrightarrow} X^{\prime}$ as weak kernel and $Y^{\prime \prime} \stackrel{1_{Y^{\prime \prime}}}{\longrightarrow} Y^{\prime \prime}$ as weak cokernel.

Choosing a kernel and a cokernel for each object in $\hat{\mathcal{C}}\left(\Delta_{1}\right)$, we obtain a kernel and a cokernel functor $\hat{\mathcal{C}}\left(\Delta_{1}\right) \Longrightarrow \hat{\mathcal{C}}$, as for any abelian category.

(2) We stipulate that the pullback resp. the pushout of an identity morphism along a morphism is chosen to be an identity morphism.

(3) We have a full and faithful functor $\mathcal{C} \longrightarrow \hat{\mathcal{C}}, X \longmapsto\left(X \stackrel{1_{X}}{\longrightarrow} X\right)$. Its image, identified with $\mathcal{C}$, consists of bijective objects.

(4) For each $X=\left(X^{\prime} \stackrel{x}{\longrightarrow} X^{\prime \prime}\right) \in \mathrm{Ob} \hat{\mathcal{C}}$, we may define objects and morphisms

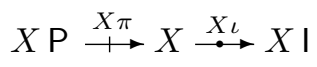

by

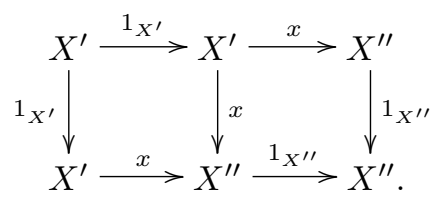

As already mentioned in (3), the objects $X \mathrm{P}$ and $X \mathrm{I}$ are bijective, and thus $\hat{\mathcal{C}}$ is Frobenius. This construction is not meant to be functorial in $\left(X^{\prime} \stackrel{x}{\longrightarrow} X^{\prime \prime}\right)$, however.

Sometimes, we write just $\iota$ for $X \iota$ and $\pi$ for $X \pi$. Note that $X \pi=1_{X}$ and $X \iota=1_{X}$ if $X \in \mathrm{ObC}$.

Remark A.2\%. Suppose given morphisms $X \stackrel{f}{\longrightarrow} Y \stackrel{g}{\longrightarrow} Z$ in $\mathcal{C}$. The following assertions are equivalent.

(i) The morphism $f$ is a weak kernel of $g$.

(ii) The morphism $g$ is a weak cokernel of $f$.

(iii) The sequence $(f, g)$ is exact at $Y$ when considered in $\hat{\mathcal{C}}$.

Proof. Ad (i) $\Longrightarrow$ (iii). Suppose that $f$ is a weak kernel of $g$. Let $K \stackrel{i}{\longrightarrow} Y$ be the kernel of $g$ in $\hat{\mathcal{C}}$. Factor $f=f^{\prime} i$. Since $f$ is a weak kernel of $g$ in $\mathcal{C}$, we may factor $(K \pi) i=u f$, from which $K \pi=u f^{\prime}$. Hence $f^{\prime}$ is epimorphic.

Ad (iii) $\Longrightarrow$ (i). Suppose $(f, g)$ to be exact at $Y$. Let $T \stackrel{t}{\longrightarrow} Y$ in $\mathcal{C}$ be such that $t g=0$. Then $t$ factors over the kernel of $g$, taken in $\hat{\mathcal{C}}$, and therefore, by projectivity of $T$ in $\hat{\mathcal{C}}$, also over $X$. 
Remark A.28. A morphism $X \stackrel{f}{\longrightarrow} Y$ in $\mathcal{C}$ is monomorphic if and only if it is a coretraction. Dually, it is epimorphic if and only if it is a retraction. ${ }^{9}$

Proof. Suppose $f$ to be monomorphic in $\mathcal{C}$. It suffices to show that $f$ is monomorphic in $\hat{\mathcal{C}}$, for then $f$ is a coretraction since $X$ is injective in $\hat{\mathcal{C}}$. Let $K \stackrel{i}{\longrightarrow} X$ be the kernel of $f$ in $\hat{\mathcal{C}}$. From $(K \pi) i f=0$, we conclude $(K \pi) i=0$ since $f$ is monomorphic in $\mathcal{C}$, and thus $K \simeq 0$ since $K \pi$ is epimorphic and $i$ is monomorphic in $\hat{\mathcal{C}}$.

Let $\mathcal{E}$ be a Frobenius category; cf. $\S$ A.2.3.

Lemma A.29. Suppose given a pure short exact sequence $X^{\prime} \stackrel{i}{\rightarrow} X \stackrel{p}{\rightarrow} X^{\prime \prime}$ in $\mathcal{E}$. In $\underline{\mathcal{E}}$, the residue class $i N$ is a weak kernel of $p N$, and the residue class $p N$ is a weak cokernel of $i N$.

Proof. By duality, it suffices to show that $i N$ is a weak kernel of $p N$. So suppose given $T \stackrel{t}{\longrightarrow} X$ in $\mathcal{E}$ with $t p \equiv_{\mathcal{B}} 0$. We have to show that there exists a morphism $T \stackrel{t^{\prime}}{\longrightarrow} X^{\prime}$ such that $t^{\prime} i \equiv \mathcal{B}$. Let $\left(T \stackrel{t}{\longrightarrow} X \stackrel{p}{\longrightarrow} X^{\prime \prime}\right)=\left(T \stackrel{u}{\longrightarrow} B \stackrel{q}{\longrightarrow} X^{\prime \prime}\right)$, where $B$ is bijective. Let $P \stackrel{\tilde{p}}{\rightarrow} B$ be the pullback of $p$ along $q$. We have a factorisation $(T \stackrel{t}{\longrightarrow} X)=(T \stackrel{v}{\longrightarrow} P \stackrel{w}{\longrightarrow} X)$. We have a factorisation $\left(X^{\prime} \stackrel{i}{\rightarrow} X\right)=$ $\left(X^{\prime} \stackrel{\tilde{i}}{\longrightarrow} P \stackrel{w}{\longrightarrow} X\right)$, moreover, $(\tilde{i}, \tilde{p})$ is a pure short exact sequence, hence split by projectivity of $B$; see Lemma A.11 and $\S A .2 .2$. Let $\tilde{i} r=1$. Then $r \tilde{i}-1 \equiv_{\mathcal{B}} 0$, since it factors over $B$. We obtain $(v r) i=v r \tilde{i} w \equiv \mathcal{B} v w=t$.

Remark A.30. The stable category $\underline{\underline{\mathcal{E}}}$ and the classical stable category $\underline{\mathcal{E}}$ of the Frobenius category $\mathcal{E}$ are weakly abelian. The stable category $\underline{\underline{\mathcal{E}}}$ carries an automorphism $\mathrm{T}$, induced by shifting a purely acyclic complex to the left by one position.

Proof. By Lemma A.8, it remains to prove that $\underline{\mathcal{E}}$ is weakly abelian. Suppose given a morphism $X \stackrel{f}{\longrightarrow} Y$ in $\mathcal{E}$. By duality, it suffices to show that the residue class of $X \stackrel{f}{\longrightarrow} Y$ in $\underline{\mathcal{E}}$ is a weak cokernel and has a weak kernel. Substituting isomorphically in $\underline{\mathcal{E}}$ by adding a bijective object to $X$, we may assume $f$ to be a pure epimorphism in $\mathcal{E}$. So we may complete to a pure short exact sequence and apply Lemma A.29.

Lemma A.31. A pure short exact sequence $X^{\prime} \stackrel{i}{\rightarrow} X \stackrel{p}{\rightarrow} X^{\prime \prime}$ in $\mathcal{E}$ is mapped via the residue class functor $N$ to a sequence in $\underline{\mathcal{E}}$ that is exact at $X$ when considered in the Freyd category $\underline{\hat{\mathcal{E}}}$ of $\underline{\mathcal{E}}$. In particular, a pure square in $\mathcal{E}$ is mapped to a weak square in $\underline{\mathcal{E}}$.

Proof. By Remark A.27, we may apply Lemma A.29.

\footnotetext{
${ }^{9}$ In particular, an abelian category is weakly abelian if and only if it is semisimple, i.e. if and only if every morphism in $\mathcal{A}$ splits. Hence the notion "weakly abelian" is slightly abusive.
} 


\section{A.6.4 HELlER OPERATORS FOR DIAGRAMS}

In Definition 1.5, the central role is attributed to the tuple $\vartheta=\left(\vartheta_{n}\right)_{n \geqslant 0}$ of isomorphisms. In the case of $\mathcal{C}$ being the stable category of a Frobenius category, such an isomorphism $\vartheta_{n}$ arises from different choices of pure monomorphisms into bijective objects. To that end, we provide a comparison lemma, which suitably organises well known facts.

Let $C$ be a category.

Given a category $\mathcal{D}$ and a full subcategory $\mathcal{U} \subseteq \mathcal{D}(C)$, we say that $\mathcal{U}$ is characteristic in $\mathcal{D}(C)$ if the image of $\mathcal{U}$ under $A(C)$ is contained in $\mathcal{U}$ for any autoequivalence $\mathcal{D} \stackrel{A}{\sim} \mathcal{D}$, and if $\mathcal{U}$ is closed under isomorphy in $\mathcal{D}(C)$, i.e. $X \simeq X^{\prime}$ in $\mathcal{D}(C)$ and $X^{\prime} \in \mathrm{Ob} \mathcal{U}$ implies $X \in \mathrm{Ob} \mathcal{U}$.

Let $\mathcal{E}$ be a Frobenius category. Denote by $\underline{\mathcal{E}}$ its classical stable category, and denote by $\mathcal{E} \stackrel{N}{\longrightarrow} \underline{\mathcal{E}}$ the residue class functor. Let $\mathcal{G} \subseteq \mathcal{E}(C)$ be a full additive subcategory. Let $\mathcal{H} \subseteq \underline{\mathcal{E}}(C)$ be a full additive characteristic subcategory such that $(\mathcal{G})(N(C)) \subseteq \mathcal{H}$.

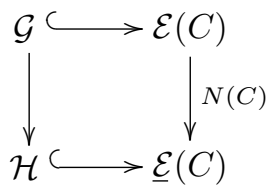

A $\boldsymbol{C}$-resolving system $I$ consists of pure short exact sequences

$$
I=\left(\left(X_{c} \stackrel{i_{X, c}}{\longrightarrow} I_{X, c} \stackrel{p_{X, c}}{\longrightarrow} \tilde{X}_{c}\right)_{c \in \mathrm{Ob} C}\right)_{X \in \mathrm{Ob} \mathcal{G}},
$$

with bijective objects $I_{X, c}$ in $\mathcal{E}$ as middle terms.

\section{Lemma A.32.}

(1) Given a C-resolving system

$$
I=\left(\left(X_{c} \stackrel{i_{X, c}}{\longrightarrow} I_{X, c} \stackrel{p_{X, c}}{\longrightarrow} \tilde{X}_{c}\right)_{c \in \mathrm{Ob} C}\right)_{X \in \mathrm{Ob} \mathcal{G}},
$$

there exists a functor

$$
\mathcal{G} \stackrel{\mathrm{T}_{I}}{\longrightarrow} \mathcal{H}
$$

that is uniquely characterised by the following properties.

On objects $X \in \mathrm{Ob} \mathcal{G} \subseteq \mathrm{Ob} \mathcal{E}(C)$, the image $X \mathrm{~T}_{I} \in \mathrm{Ob} \mathcal{H} \subseteq \mathrm{Ob} \underline{\mathcal{E}}(C)$ is characterised as follows. 
Homology, Homotopy and Applications, vol. 9(2), 2007

(*) For any $(c \stackrel{\gamma}{\longrightarrow} d) \in C$, there exist

- a representative $\left(X \mathrm{~T}_{I}\right)_{\gamma}^{\sim}$ in $\mathcal{E}$ of the evaluation $\left(X \mathrm{~T}_{I}\right)_{c} \stackrel{\left(X \mathrm{~T}_{I}\right)_{\gamma}}{\longrightarrow}\left(X \mathrm{~T}_{I}\right)_{d}$ in $\underline{\mathcal{E}}$ at $c \stackrel{\gamma}{\longrightarrow} d$ of the diagram $X \mathrm{~T}_{I} \in \mathrm{Ob} \mathcal{H} \subseteq \mathrm{Ob} \underline{\mathcal{E}}(C)$, and

- a morphism $I_{X, c} \longrightarrow I_{X, d}$ in $\mathcal{E}$

such that

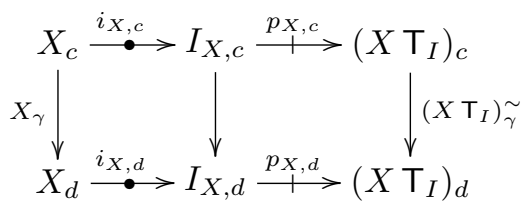

is a morphism of pure short exact sequences.

On morphisms $(X \stackrel{f}{\longrightarrow} Y) \in \mathcal{G} \subseteq \mathcal{E}(C)$, the image $\left(X \mathrm{~T}_{I} \stackrel{f \mathrm{~T}_{I}}{\longrightarrow} Y \mathrm{~T}_{I}\right) \in \mathcal{H} \subseteq$ $\underline{\mathcal{E}}(C)$ is characterised as follows.

(**) For any $c \in \mathrm{Ob} C$, there exist

- a representative $\left(f \mathrm{~T}_{I}\right)_{c}^{\sim}$ in $\mathcal{E}$ of the evaluation $\left(X \mathrm{~T}_{I}\right)_{c} \stackrel{\left(f \mathrm{~T}_{I}\right)_{c}}{\longrightarrow}\left(Y \mathrm{~T}_{I}\right)_{c}$ in $\underline{\mathcal{E}}$ at $c$ of the diagram morphism $\left(X \mathrm{~T}_{I} \stackrel{f \mathrm{~T}_{I}}{\longrightarrow} Y \mathrm{~T}_{I}\right) \in \mathcal{H} \subseteq \underline{\mathcal{E}}(C)$, and

- a morphism $I_{X, c} \longrightarrow I_{Y, c}$ in $\mathcal{E}$

such that

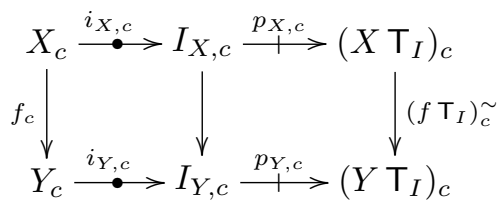

is a morphism of pure short exact sequences.

(2) Given C-resolving systems

$$
\begin{aligned}
I & =\left(\left(X_{c} \stackrel{i_{X, c}}{\longrightarrow} I_{X, c} \stackrel{p_{X, c}}{\longrightarrow} \tilde{X}_{c}\right)_{c \in \mathrm{Ob} C}\right)_{X \in \mathrm{Ob} \mathcal{G}}, \\
I^{\prime} & =\left(\left(X_{c} \stackrel{i_{X, c}^{\prime}}{\longrightarrow} I_{X, c}^{\prime} \stackrel{p_{X, c}^{\prime}}{\longrightarrow} \tilde{X}_{c}^{\prime}\right)_{c \in \mathrm{Ob} C}\right)_{X \in \mathrm{Ob} \mathcal{G}},
\end{aligned}
$$

there exists an isomorphism

$$
\mathrm{T}_{I} \stackrel{\alpha_{I, I^{\prime}}}{\sim} \mathrm{T}_{I^{\prime}}
$$

that is uniquely characterised by the following property. 
Homology, Homotopy and Applications, vol. 9(2), 2007

$(* * *) \quad$ For any $X \in \mathrm{Ob} \mathcal{G} \subseteq \mathrm{Ob} \mathcal{E}(C)$ and for any $c \in \mathrm{Ob} C$, there exist

- a representative $\left(X \alpha_{I, I^{\prime}}\right)^{\sim}$ in $\mathcal{E}$ of the evaluation $\left(X \mathrm{~T}_{I}\right)_{c}^{\left(X \alpha_{I, I^{\prime}}\right)_{c}}\left(X \mathrm{~T}_{I^{\prime}}\right)_{c}$ in $\underline{\mathcal{E}}$ at $c$ of the evaluation $X \mathrm{~T}_{I} \stackrel{X \alpha_{I, I^{\prime}}}{\longrightarrow} X \mathrm{~T}_{I^{\prime}}$ in $\mathcal{H} \subseteq \underline{\mathcal{E}}(C)$ of $\alpha_{I, I^{\prime}}$ at $X$, and

- a morphism $I_{X, c} \longrightarrow I_{X, c}^{\prime}$ in $\mathcal{E}$

such that

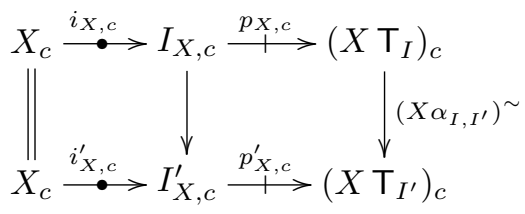

is a morphism of pure short exact sequences.

Proof. Let us first assume that $\mathcal{H}=\underline{\mathcal{E}}(C)$. Having proven all assertions in this case, it then finally will remain to be shown that given $\mathcal{H} \subseteq \underline{\mathcal{E}}(C)$ and a $C$-resolving system $I$, we have $X \mathrm{~T}_{I} \in \mathrm{Ob} \mathcal{H} \subseteq \mathrm{Ob} \underline{\mathcal{E}}(C)$ for $X \in \mathrm{Ob} \mathcal{G}$.

We remark that starting from a morphism $U \stackrel{u}{\longrightarrow} U^{\prime}$ in $\mathcal{E}$ and from chosen pure short exact sequences $(U, B, V)$ and $\left(U^{\prime}, B^{\prime}, V^{\prime}\right)$ with bijective middle term $B$ resp. $B^{\prime}$, we may define a morphism $V \stackrel{v}{\longrightarrow} V^{\prime}$ in $\underline{\mathcal{E}}$ by the existence of a morphism

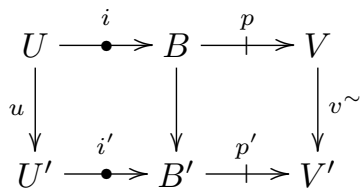

of pure short exact sequences in $\mathcal{E}$, where $V \stackrel{v}{\longrightarrow} V^{\prime}$ is the image in $\underline{\mathcal{E}}$ of the morphism $V \stackrel{v^{\sim}}{\longrightarrow} V^{\prime}$ in $\mathcal{E}$.

Ad (1). Given $X \in \mathrm{Ob} \mathcal{G}$, we define $X \mathrm{~T}_{I} \in \underline{\mathcal{E}}(C)$ at the morphism $c \stackrel{\gamma}{\longrightarrow} d$ of $C$ by the diagram in (*). The characterisation $(*)$ shows that $X \mathrm{~T}_{I}$ is in fact in $\mathrm{Ob} \underline{\mathcal{E}}(C)$.

Given a morphism $X \stackrel{f}{\longrightarrow} Y$ in $\mathcal{G}$, we define the morphism $X \mathrm{~T}_{I} \stackrel{f \mathrm{~T}_{I}}{\longrightarrow} Y \mathrm{~T}_{I}$ in $\underline{\mathcal{E}}(C)$ at $c \in \mathrm{Ob} C$ by the diagram in $(* *)$. Combining $(*)$ and $(* *)$, we see that $f \mathrm{~T}_{I}$ is in fact in $\underline{\mathcal{E}}(C)$. From $(* *)$ we conclude that $\mathrm{T}_{I}$ is indeed a functor.

Ad (2). Given $X \in \mathrm{Ob} \mathcal{G}$, we define $X \mathrm{~T}_{I} \stackrel{X \alpha_{I, I^{\prime}}}{\longrightarrow} X \mathrm{~T}_{I^{\prime}}$ at $c \in \mathrm{Ob} C$ by the diagram in $(* * *)$.

Combining $(* * *)$ and $(*)$, we see that $X \mathrm{~T}_{I} \stackrel{X \alpha_{I, I^{\prime}}}{\longrightarrow} X \mathrm{~T}_{I^{\prime}}$ is indeed in $\underline{\mathcal{E}}(C)$. Combining $(* * *)$ and $(* *)$, we see that $\alpha_{I, I^{\prime}}$ is indeed a transformation.

Suppose given resolving systems $I, I^{\prime}$ and $I^{\prime \prime}$. The characterisation of $\alpha_{I, I^{\prime}}$ etc. implies that $\alpha_{I, I^{\prime}} \alpha_{I^{\prime}, I^{\prime \prime}}=\alpha_{I, I^{\prime \prime}}$ and that $\alpha_{I, I}=1_{\mathrm{T}_{I}}$. Hence in particular, $\alpha_{I, I^{\prime}} \alpha_{I^{\prime}, I}=1_{\mathrm{T}_{I}}$ and $\alpha_{I^{\prime}, I} \alpha_{I, I^{\prime}}=1_{\mathrm{T}_{I^{\prime}}}$, and so $\alpha_{I, I^{\prime}}$ is an isomorphism from $\mathrm{T}_{I}$ to $\mathrm{T}_{I^{\prime}}$. 
Consider the case $C=\Delta_{0}$, i.e. the terminal category, letting $\mathcal{G}=\mathcal{E}\left(\Delta_{0}\right)=\mathcal{E}$ and letting $\mathcal{H}=\underline{\mathcal{E}}\left(\Delta_{0}\right)=\underline{\mathcal{E}}$. For a $\Delta_{0}$-resolving system $J$, we obtain a functor $\mathcal{E} \stackrel{\mathrm{T}_{J}}{\longrightarrow} \underline{\mathcal{E}}$ that factors as

$$
\left(\mathcal{E} \stackrel{\mathrm{T}_{J}}{\longrightarrow} \underline{\mathcal{E}}\right)=\left(\mathcal{E} \stackrel{N}{\longrightarrow} \underline{\mathcal{E}} \stackrel{\overline{\mathrm{T}}_{J}}{\longrightarrow} \underline{\mathcal{E}}\right) .
$$

In fact, for a morphism $b$ that factors over a bijective object $B$, we can choose 0 as a representative of $b \mathrm{~T}_{J}$, inserting the pure short exact sequence $(B, B, 0)$. Moreover $\overline{\mathrm{T}}_{J}$ is an equivalence, for it is full; it is faithful, using the dual of the argument just given; and dense, since given a morphism of short exact sequences in $\mathcal{E}$ with bijective middle terms and an identity on the kernels, the morphism on the cokernels is a homotopism.

Now return to the general case $\mathcal{H} \subseteq \underline{\mathcal{E}}(C)$. Let $J^{\prime}$ be a $C$-resolving system consisting of pure short exact sequences with bijective middle term that already occur in the chosen $\Delta_{0}$-resolving system $J$. Then, for $X \in \mathrm{Ob} \mathcal{G}$, we have $X \mathrm{~T}_{J^{\prime}}=$ $X(N(C))\left(\overline{\mathrm{T}}_{J}(C)\right)$. Since $X(N(C)) \in \mathrm{Ob} \mathcal{H}$ by assumption, and since, moreover, $\mathcal{H}$ is assumed to be a characteristic subcategory of $\underline{\mathcal{E}}(C)$, we conclude that $X(N(C))\left(\overline{\mathrm{T}}_{J}(C)\right)=X \mathrm{~T}_{J^{\prime}}$ is in Ob $\mathcal{H}$. Finally, let $I$ be an arbitrary $C$-resolving system. We have $X \mathrm{~T}_{I} \stackrel{\alpha_{I, J^{\prime}}}{\sim} X \mathrm{~T}_{J^{\prime}}$ in $\underline{\mathcal{E}}(C)$, and thus $X \mathrm{~T}_{J^{\prime}} \in \mathrm{Ob} \mathcal{H}$ implies $X \mathrm{~T}_{I} \in$ Ob $\mathcal{H}$, since a characteristic subcategory of $\underline{\mathcal{E}}(C)$ is, by definition, closed under isomorphy.

\section{A.7. Pointwise pullback and pushout}

Suppose given an abelian category $\mathcal{A}$, a poset $E$ and an element $\varepsilon \in E$. Let $E^{\varepsilon}:=E \sqcup\left\{\varepsilon^{\prime}\right\}$ be the poset defined by requiring that $\varepsilon \leqslant \varepsilon^{\prime}$, that $\alpha \nless \varepsilon^{\prime}$ whenever $\alpha \notin \varepsilon$ and that $\varepsilon^{\prime} \nless \alpha$ for all $\alpha \in E$; and the remaining relations within $E \subseteq E^{\varepsilon}$ inherited from $E$. We define the pushout at $\varepsilon$

$$
\begin{aligned}
\mathcal{A}\left(E^{\varepsilon}\right) & \stackrel{(-) \uparrow^{(=)}}{\longrightarrow} \mathcal{A}(E) \\
X^{\prime} & \longmapsto X \uparrow^{x^{\prime}},
\end{aligned}
$$

where $X:=\left.X^{\prime}\right|_{E}$, and $\left(X_{\varepsilon}^{\prime} \stackrel{x^{\prime}}{\longrightarrow} X_{\varepsilon^{\prime}}^{\prime}\right)=\left(X_{\varepsilon}^{\prime} \stackrel{X_{\varepsilon^{\prime} / \varepsilon}^{\prime}}{\longrightarrow} X_{\varepsilon^{\prime}}^{\prime}\right)$; and a transformation

$$
\left.X^{\prime}\right|_{E}=X \stackrel{i=i X^{\prime}}{\longrightarrow} X \uparrow^{x^{\prime}}
$$

natural in $X^{\prime}$, by the following construction. Abbreviating $X \uparrow x^{\prime}$ by $\tilde{X}$, we let

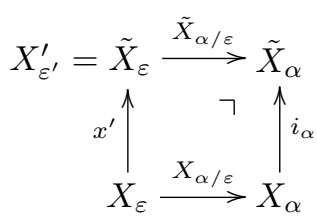

for $\alpha \in E$ with $\varepsilon \leqslant \alpha$. If $\varepsilon \not \alpha$, we let $\tilde{X}_{\alpha}=X_{\alpha}$ and $i_{\alpha}=1_{X_{\alpha}}$. 
Given $\alpha \leqslant \beta$ in $E$, we let

$$
\begin{aligned}
& \left(\tilde{X}_{\alpha} \stackrel{\tilde{X}_{\beta / \alpha}}{\longrightarrow} \tilde{X}_{\beta}\right) \text { be induced by pushout if } \varepsilon \leqslant \alpha \leqslant \beta \text {, } \\
& \left(\tilde{X}_{\alpha} \underset{\tilde{X}_{\beta / \alpha}}{\longrightarrow} \tilde{X}_{\beta}\right):=\left(X_{\alpha} \stackrel{X_{\beta / \alpha}}{\longrightarrow} X_{\beta} \stackrel{i_{\beta}}{\longrightarrow} \tilde{X}_{\beta}\right) \text { if } \varepsilon \not \nless \alpha \text {, but } \varepsilon \leqslant \beta \text {, } \\
& \left(\tilde{X}_{\alpha} \stackrel{\tilde{X}_{\beta / \alpha}}{\longrightarrow} \tilde{X}_{\beta}\right):=\left(X_{\alpha} \stackrel{X_{\beta / \alpha}}{\longrightarrow} X_{\beta}\right) \quad \text { if } \varepsilon \not<\beta \text {. }
\end{aligned}
$$

The morphism $X \stackrel{i}{\longrightarrow} X \uparrow^{x^{\prime}}$ is the solution to the following universal problem. Suppose given a morphism $X \stackrel{f}{\longrightarrow} Y$ in $\mathcal{A}(E)$ such that at $\varepsilon \in E$ we have a factorisation

$$
\left(X_{\varepsilon} \stackrel{f_{\varepsilon}}{\longrightarrow} Y_{\varepsilon}\right)=\left(X_{\varepsilon} \stackrel{x^{\prime}}{\longrightarrow} X_{\varepsilon^{\prime}}^{\prime} \longrightarrow Y_{\varepsilon}\right) .
$$

Then there is a unique morphism $X \uparrow^{x^{\prime}} \stackrel{g}{\longrightarrow} Y$ such that

$$
(X \stackrel{f}{\longrightarrow} Y)=\left(X \stackrel{i}{\longrightarrow} X \uparrow^{x^{\prime}} \stackrel{g}{\longrightarrow} Y\right) .
$$

Dually, let $E_{\varepsilon}:=E \sqcup\left\{\varepsilon^{\prime}\right\}$ be the poset defined by requiring that $\varepsilon \geqslant \varepsilon^{\prime}$, that $\alpha \nsupseteq \varepsilon^{\prime}$ whenever $\alpha \ngtr \varepsilon$ and that $\varepsilon^{\prime} \ngtr \alpha$ for all $\alpha \in E$; the remaining relations within $E \subseteq E_{\varepsilon}$ being inherited from $E$. We define the pullback at $\varepsilon$

$$
\begin{aligned}
\mathcal{A}\left(E_{\varepsilon}\right) & \stackrel{(-) \uparrow(=)}{\longrightarrow} \mathcal{A}(E) \\
X^{\prime} & \longmapsto X \uparrow_{x^{\prime}},
\end{aligned}
$$

where $X:=\left.X^{\prime}\right|_{E}$, and $\left(X_{\varepsilon^{\prime}}^{\prime} \stackrel{x^{\prime}}{\longrightarrow} X_{\varepsilon}^{\prime}\right)=\left(X_{\varepsilon^{\prime}}^{\prime} \stackrel{X_{\varepsilon / \varepsilon^{\prime}}^{\prime}}{\longrightarrow} X_{\varepsilon}^{\prime}\right)$; and a transformation

$$
\left.X^{\prime}\right|_{E}=X \longleftarrow p=p X^{\prime}-X \uparrow x^{\prime},
$$

natural in $X^{\prime}$, being the solution to the dual universal problem.

\section{A.8. 1-epimorphic functors}

Let $\mathcal{C} \stackrel{F}{\longrightarrow} \mathcal{D}$ be a functor between categories $\mathcal{C}$ and $\mathcal{D}$.

Definition A.33. The functor $\mathcal{C} \stackrel{F}{\longrightarrow} \mathcal{D}$ is 1-epimorphic if the induced functor "restriction along $F$ "

$$
\llbracket \mathcal{C}, \mathcal{E} \rrbracket \stackrel{F(-)}{\longleftarrow} \llbracket \mathcal{D}, \mathcal{E} \rrbracket
$$

is full and faithful for any category $\mathcal{E}$. In particular, given functors $\mathcal{D} \underset{H}{\stackrel{G}{\longrightarrow}} \mathcal{E}$ with $F G \simeq F H$, we can conclude that $G \simeq H$; whence the notion of 1-epimorphy.

Remark A.34. Suppose given a diagram of categories and functors

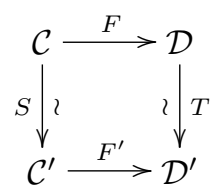

with equivalences $S$ and $T$, and with $F T \simeq S F^{\prime}$. Then $F$ is 1-epimorphic if and only if $F^{\prime}$ is 1-epimorphic. 
Let $C, C^{\prime} \in \mathrm{Ob} \mathcal{C}$. An $\boldsymbol{F}$-epizigzag (resp. an $\boldsymbol{F}$-monozigzag) $C \stackrel{u}{\sim} C^{\prime}$ is a finite sequence of morphisms

$$
C=C_{0} \stackrel{u_{0}}{\longrightarrow} Z_{0} \stackrel{u_{0}^{\prime}}{\longrightarrow} C_{1} \stackrel{u_{1}}{\longrightarrow} Z_{1} \cdots \stackrel{u_{k-2}^{\prime}}{\stackrel{u_{k-1}}{\longrightarrow}} Z_{k-1} \stackrel{u_{k-1}^{\prime}}{\stackrel{u_{k}}{\longrightarrow}} C_{k}=C^{\prime}
$$

in $\mathcal{C}$ of length $k \geqslant 0$ such that $u_{i}^{\prime} F$ is an isomorphism for all $i \in[0, k]$, and such that

$$
u F:=\left(u_{0} F\right)\left(u_{0}^{\prime} F\right)^{-}\left(u_{1} F\right)\left(u_{1}^{\prime} F\right)^{-} \cdots\left(u_{k-1} F\right)\left(u_{k-1}^{\prime} F\right)^{-}: C F \longrightarrow C^{\prime} F
$$

is a retraction (resp. a coretraction) in $\mathcal{D}$.

Lemma A.35. Suppose the functor

$$
\mathcal{C} \stackrel{F}{\longrightarrow} \mathcal{D}
$$

to be dense, and to satisfy the following condition (C).

(C) Given objects $C, C^{\prime} \in \mathrm{ObC}$ and a morphism $C F \stackrel{d}{\longrightarrow} C^{\prime} F$ in $\mathcal{D}$, there exists an $F$-epizigzag $C_{\mathrm{s}} \stackrel{c_{\mathrm{s}}}{\rightarrow} C$, an $F$-monozigzag $C^{\prime} \stackrel{c_{\mathrm{t}}^{\prime}}{\rightarrow} C_{\mathrm{t}}^{\prime}$ and a morphism $C_{\mathrm{s}} \stackrel{c}{\longrightarrow} C_{\mathrm{t}}^{\prime}$ such that

$$
\left(C_{s} F \stackrel{c_{s} F}{\longrightarrow} C F \stackrel{d}{\longrightarrow} C^{\prime} F \stackrel{c_{t}^{\prime} F}{\longrightarrow} C_{t}^{\prime} F\right)=\left(C_{s} F \stackrel{c F}{\longrightarrow} C_{t}^{\prime} F\right) .
$$

Then $F$ is 1-epimorphic.

Proof. Since $F$ is dense, Remark A.34 allows the assumption that $F$ is surjective on objects, i.e. $(\mathrm{Ob} \mathcal{C}) F=\mathrm{Ob} \mathcal{D}$.

Let us prove that $\mathcal{E}(\mathcal{C}) \stackrel{F(-)}{\leftarrow} \mathcal{E}(\mathcal{D})$ is faithful. Suppose given functors $\mathcal{C} \stackrel{F}{\longrightarrow}$ $\mathcal{D} \underset{H}{\stackrel{G}{\longrightarrow}} \mathcal{E}$ and morphisms $G \stackrel{\gamma}{\longrightarrow} H$ and $G \stackrel{\gamma^{\prime}}{\longrightarrow} H$ such that $F \gamma=F \gamma^{\prime}$. Given $D \in$ Ob $\mathcal{D}$, we have to show that $D \gamma=D \gamma^{\prime}$. Writing $D=C F$ for some $C \in \mathrm{Ob} \mathcal{C}$, this follows from $D \gamma=C F \gamma=C F \gamma^{\prime}=D \gamma^{\prime}$.

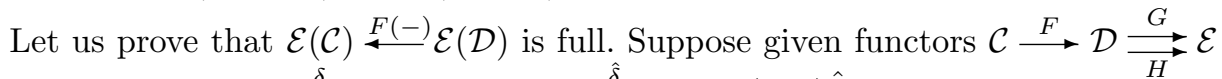
and a morphism $F G \stackrel{\delta}{\longrightarrow} F H$. Define $G \stackrel{\hat{\delta}}{\longrightarrow} H$ by $(C F) \hat{\delta}:=C \delta$.

We have to prove that $D \hat{\delta}$ is a well defined morphism for $D \in \mathrm{Ob} \mathcal{D}$. So suppose that $D=C F=C^{\prime} F$. We have to show that $C \delta=C^{\prime} \delta$. By assumption (C), applied to $d=1_{D}=1_{C F}=1_{C^{\prime} F}$, there exist an $F$-epizigzag $C_{\mathrm{s}} \stackrel{c_{\mathrm{S}}}{\sim} C$, an $F$-monozigzag $C^{\prime} \stackrel{c_{\mathrm{t}}^{\prime}}{\rightarrow} C_{\mathrm{t}}^{\prime}$ and a morphism $C_{\mathrm{s}} \stackrel{c}{\longrightarrow} C_{\mathrm{t}}^{\prime}$ such that $\left(c_{\mathrm{s}} F\right)\left(c_{\mathrm{t}}^{\prime} F\right)=c F$. We obtain

$$
\begin{aligned}
\left(c_{\mathrm{s}} F G\right)(C \delta)\left(c_{\mathrm{t}}^{\prime} F H\right) & =\left(C_{\mathrm{s}} \delta\right)\left(c_{\mathrm{s}} F H\right)\left(c_{\mathrm{t}}^{\prime} F H\right) \\
& =\left(C_{\mathrm{s}} \delta\right)(c F H) \\
& =(c F G)\left(C_{\mathrm{t}}^{\prime} \delta\right) \\
& =\left(c_{\mathrm{s}} F G\right)\left(c_{\mathrm{t}}^{\prime} F G\right)\left(C_{\mathrm{t}}^{\prime} \delta\right) \\
& =\left(c_{\mathrm{s}} F G\right)\left(C^{\prime} \delta\right)\left(c_{\mathrm{t}}^{\prime} F H\right),
\end{aligned}
$$

from which $C \delta=C^{\prime} \delta$ by epimorphy of $c_{\mathrm{s}} F G$ and by monomorphy of $c_{\mathrm{t}}^{\prime} F H$.

We have to prove that $\hat{\delta}$ is natural. Suppose given $C F \stackrel{d}{\longrightarrow} C^{\prime} F$ in $\mathcal{D}$ for some $C, C^{\prime} \in \mathrm{Ob} \mathcal{C}$. We have to show that $(d G)\left(\left(C^{\prime} F\right) \hat{\delta}\right)=((C F) \hat{\delta})(d H)$, i.e. that $(d G)\left(C^{\prime} \delta\right)=(C \delta)(d H)$. By assumption $(\mathrm{C})$, there exist an $F$-epizigzag $C_{\mathrm{s}} \stackrel{c_{\mathrm{s}}}{\sim} C$, an 
$F$-monozigzag $C^{\prime} \stackrel{c_{\mathrm{t}}^{\prime}}{\rightarrow} C_{\mathrm{t}}^{\prime}$ and a morphism $C_{\mathrm{s}} \stackrel{c}{\longrightarrow} C_{\mathrm{t}}^{\prime}$ such that $\left(c_{\mathrm{s}} F\right) d\left(c_{\mathrm{t}}^{\prime} F\right)=c F$. We obtain

$$
\begin{aligned}
\left(c_{\mathrm{s}} F G\right)(d G)\left(C^{\prime} \delta\right)\left(c_{\mathrm{t}}^{\prime} F H\right) & =\left(c_{\mathrm{s}} F G\right)(d G)\left(c_{\mathrm{t}}^{\prime} F G\right)\left(C_{\mathrm{t}}^{\prime} \delta\right) \\
& =(c G F)\left(C_{\mathrm{t}}^{\prime} \delta\right) \\
& =\left(C_{\mathrm{s}} \delta\right)(c F H) \\
& =\left(C_{\mathrm{s}} \delta\right)\left(c_{\mathrm{s}} F H\right)(d H)\left(C_{\mathrm{t}}^{\prime} F H\right) \\
& =\left(c_{\mathrm{s}} F G\right)(C \delta)(d H)\left(c_{\mathrm{t}}^{\prime} F H\right),
\end{aligned}
$$

from which $(d G)\left(C^{\prime} \delta\right)=(C \delta)(d H)$ by epimorphy of $c_{\mathrm{s}} F G$ and by monomorphy of $c_{\mathrm{t}}^{\prime} F H$.

Corollary A.36. If $\mathcal{C} \stackrel{F}{\longrightarrow} \mathcal{D}$ is a functor such that (i), (ii) hold, then $F$ is 1epimorphic.

(i) For all morphisms $D \stackrel{d}{\longrightarrow} D^{\prime}$ in $\mathcal{D}$, there is a morphism $C \stackrel{c}{\longrightarrow} C^{\prime}$ in $\mathcal{C}$ such that

$$
\left(C \stackrel{c}{\longrightarrow} C^{\prime}\right) F=\left(D \stackrel{d}{\longrightarrow} D^{\prime}\right) .
$$

(ii) For any $C, C^{\prime} \in \mathrm{ObC}$ such that $C F=C^{\prime} F$, there exists a finite sequence of morphisms

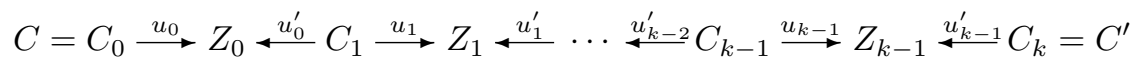

from $C$ to $C^{\prime}$ such that $u_{i} F=u_{i}^{\prime} F=1_{C F}=1_{C^{\prime} F}$ for all $i \in[0, k]$.

Proof. The functor $F$ is dense, even surjective on objects, because identities have inverse images under $F$. To fulfill condition (C) of Lemma A.35, given objects $C, C^{\prime} \in \mathrm{ObC}$ and a morphism $C F \stackrel{d}{\longrightarrow} C^{\prime} F$ in $\mathcal{D}$, we may take some morphism $C_{\mathrm{s}} \stackrel{c}{\longrightarrow} C_{\mathrm{t}}^{\prime}$ in $\mathcal{C}$ such that $\left(C_{\mathrm{s}} \stackrel{c}{\longrightarrow} C_{\mathrm{t}}^{\prime}\right) F=\left(C F \stackrel{d}{\longrightarrow} C^{\prime} F\right)$, we may take for $c_{\mathrm{s}}$ a sequence as given by assumption because of $C_{\mathrm{s}} F=C F$, and we may take for $c_{\mathrm{t}}$ a sequence as given by assumption because of $C_{\mathrm{t}}^{\prime} F=C^{\prime} F$.

Corollary A.37. If $\mathcal{C} \stackrel{F}{\longrightarrow} \mathcal{D}$ is a full and dense functor, then $F$ is 1-epimorphic.

Proof. In fact, in condition (C) of Lemma A.35, we may take an F-monozigzag and an $F$-epizigzag of length 0 .

\section{References}

[1] H.J. Baues and F. Muro, The characteristic cohomology class of a triangulated category, preprint, arXiv:math.KT/0505540, 2005.

[2] A. Beligiannis, On the Freyd categories of an additive category, Homology Homotopy Appl. 2 (11), (2000) 147-185.

[3] J. Bernstein, A.A. Beilinson and P. Deligne, Faisceaux pervers, Astérisque 100, 1982.

[4] P. Deligne, Catégories spectrales, manuscript, 1968. 
[5] P. Deligne, Décompositions dans la catégorie dérivée, Proc. Sympos. Pure Math. 55, (1994) 115-128.

[6] A.D. Elmendorf, A simple formula for cyclic duality, Proc. Amer. Math. Soc. 118 (3), (1993) 709-711.

[7] J. Franke, Uniqueness theorems for certain triangulated categories possessing an Adams spectral sequence, preprint, http://www.math.uiuc.edu/ K-theory/0139, 1996.

[8] P. Freyd, Stable Homotopy, Proc. Conf. Categorical Algebra, La Jolla, CA, Springer, (1965) 121-172.

[9] P. Gabriel, Des catégories abéliennes, Bull. Soc. Math. France 90, (1962) 323-448.

[10] A. GrothendieCK, Les Dérivateurs, http://www.math.jussieu.fr/ $\sim$ maltsin/groth/Derivateurs.html, 1990.

[11] D. HAPPEL, Triangulated Categories in the Representation Theory of Finite Dimensional Algebras, LMS Lecture Notes 119, Cambridge University Press 1988.

[12] A. Heller, Stable homotopy categories, Bull. Amer. Math. Soc. 74, (1968) 28-63.

[13] A. Heller, Homotopy theories, Mem. Amer. Math. Soc. 383, 1988.

[14] L. Illusie, Complexe cotangent et déformations. I., Springer Lecture Notes 239, 1971 .

[15] L. Illusie, Catégories dérivées et dualité: travaux de J. L. Verdier, Enseig. Math. 36, (1990) 369-391.

[16] L. ILlusie, Perversité et variation, Manuscripta Math. 112, (2003) 271-295.

[17] B. KELLER, Chain complexes and stable categories, Manuscripta Math. 67, (1990) 379-417.

[18] B. Keller, Derived categories and universal problems, Comm. Algebra 19 (3), (1991) 699-747.

[19] B. Keller, Deriving DG categories, Ann. scient. Éc. Norm. Sup. 4ème série, t. 27, (1994) 63-102.

[20] M. KÜNZER, Nonisomorphic Verdier octahedra on the same base, preprint, arXiv:0708.0151v2, 2007.

[21] G. Laumon, Sur la catégorie derivée des D-modules filtrés, Algebraic Geometry, (Tokyo-Kyoto), Springer Lecture Notes 1016, (1983) 151-237.

[22] G. Maltsiniotis, La K-théorie d'un dérivateur triangulé, preprint, http: //www.math.jussieu.fr/ maltsin, 2002.

[23] A. Neeman, The derived category of an exact category, J. Algebra 135, (1990) 388-394.

[24] A. NeEman, Some new axioms for triangulated categories, J. Algebra 139, (1991) 221-255.

[25] A. NeEman, The K-theory of triangulated categories, Handbook of K-theory (eds. E.M. Friedlander and D.R. GRAYSON), Springer, (2005) 1011-1078. 
[26] D. Puppe, On the formal structure of stable homotopy, Coll. on Algebraic Topology, Aarhus, (1962) 65-71.

[27] D. Quillen, Higher algebraic K-theory: I, Proc. Conf., Battelle Memorial Inst., Seattle, WA., Springer Lecture Notes 341, (1973) 85-147.

[28] J.L. Verdier, Des catégories dérivées des catégories abéliennes (written 1967), Astérisque 239, 1996.

[29] J.L. VerdiER, Catégories Derivées (written 1963), SGA 4 1/2, Springer Lecture Notes 569, (1977) 262-311.

[30] F. Waldhausen, Algebraic $K$-theory of spaces, Algebraic and Geometric Topology, Springer Lecture Notes 1126, (1985) 318-419.

[31] C.A. Weibel, History of homological algebra, preprint, http://www.math. uiuc.edu/K-theory/0245, 1997.

Matthias Künzer kuenzer@math.rwth-aachen.de

Lehrstuhl D für Mathematik

RWTH Aachen

Templergraben 64

D-52062 Aachen

Germany

This article is available at http://intlpress.com/HHA/v9/n2/a10 